\title{
Iron behaving badly: inappropriate iron chelation as a major contributor to the aetiology of vascular and other progressive inflammatory and degenerative diseases

\author{
Douglas B Kell*
}

Address: School of Chemistry and Manchester Interdisciplinary Biocentre, The University of Manchester, 131 Princess St, Manchester, M1 7DN, UK

E-mail: Douglas B Kell* - dbk@manchester.ac.uk

${ }^{*}$ Corresponding author

Published: 08 January 2009

BMC Medical Genomics 2009, 2:2 doi: 10.1/86/1755-8794-2-2
Received: 2 September 2008

Accepted: 8 January 2009

This article is available from: http://www.biomedcentral.com/1755-8794/2/2

(C) 2009 Kell; licensee BioMed Central Ltd.

This is an Open Access article distributed under the terms of the Creative Commons Attribution License (http://creativecommons.org/licenses/by/2.0), which permits unrestricted use, distribution, and reproduction in any medium, provided the original work is properly cited.

\begin{abstract}
Background: The production of peroxide and superoxide is an inevitable consequence of aerobic metabolism, and while these particular 'reactive oxygen species' (ROSs) can exhibit a number of biological effects, they are not of themselves excessively reactive and thus they are not especially damaging at physiological concentrations. However, their reactions with poorly liganded iron species can lead to the catalytic production of the very reactive and dangerous hydroxyl radical, which is exceptionally damaging, and a major cause of chronic inflammation.
\end{abstract}

Review: We review the considerable and wide-ranging evidence for the involvement of this combination of (su)peroxide and poorly liganded iron in a large number of physiological and indeed pathological processes and inflammatory disorders, especially those involving the progressive degradation of cellular and organismal performance. These diseases share a great many similarities and thus might be considered to have a common cause (i.e. iron-catalysed free radical and especially hydroxyl radical generation).

The studies reviewed include those focused on a series of cardiovascular, metabolic and neurological diseases, where iron can be found at the sites of plaques and lesions, as well as studies showing the significance of iron to aging and longevity. The effective chelation of iron by natural or synthetic ligands is thus of major physiological (and potentially therapeutic) importance. As systems properties, we need to recognise that physiological observables have multiple molecular causes, and studying them in isolation leads to inconsistent patterns of apparent causality when it is the simultaneous combination of multiple factors that is responsible.

This explains, for instance, the decidedly mixed effects of antioxidants that have been observed, since in some circumstances (especially the presence of poorly liganded iron) molecules that are nominally antioxidants can actually act as pro-oxidants. The reduction of redox stress thus requires suitable levels of both antioxidants and effective iron chelators. Some polyphenolic antioxidants may serve both roles.

Understanding the exact speciation and liganding of iron in all its states is thus crucial to separating its various pro- and anti-inflammatory activities. Redox stress, innate immunity and pro- (and some anti-)inflammatory cytokines are linked in particular via signalling pathways involving NF-kappaB and $\mathrm{p} 38$, with the oxidative roles of iron here seemingly involved upstream of the lkappaB kinase (IKK) reaction. In a number of cases it is possible to identify mechanisms by which ROSs and poorly 
liganded iron act synergistically and autocatalytically, leading to 'runaway' reactions that are hard to control unless one tackles multiple sites of action simultaneously. Some molecules such as statins and erythropoietin, not traditionally associated with anti-inflammatory activity, do indeed have 'pleiotropic' anti-inflammatory effects that may be of benefit here.

Conclusion: Overall we argue, by synthesising a widely dispersed literature, that the role of poorly liganded iron has been rather underappreciated in the past, and that in combination with peroxide and superoxide its activity underpins the behaviour of a great many physiological processes that degrade over time. Understanding these requires an integrative, systems-level approach that may lead to novel therapeutic targets.

\section{Background and preamble}

The 'balkanisation' of the literature is in part due to the amount of it (some 25,000 journals with presently 2.5 million peer-reviewed papers per year, i.e. 5 per minute [1]), with a number http://www.nlm.nih.gov/ bsd/medline_cit_counts_yr_pub.html increasing by something approaching 2 per minute at PubMed/Medline alone. In addition, the disconnect between the papers in the literature (usually as pdf files) and the metadata describing them (author, journal, year, pages, etc) is acute and badly needs filling [2]. Without solving this problem, and without automation of the processes of reading, interpreting and exploiting this literature and its metadata in a digital format, we cannot make use of the existing tools for text mining and natural language processing (e.g. [3-5]), for joining disparate concepts [6], for literature-based discovery (e.g. [7-11], and for studies of bibliometrics [12, 13], literature dynamics [14], knowledge domains [15], detecting republication [16] and so on. Until we recognise these possibilities we are unlikely to seek to realise them.

The present article (and see [17] for a preprint) serves to show some of the benefits than can accrue from a more overarching view of the otherwise highly disparate literature in a particular domain (see also [18]), but was done 'the hard way', i.e. with a few bibliographic and bibliometric tools but without the kind of automation implied above. For the record, the main tools used (see a review in [2]) were Web of Knowledge and Scopus for literature and citation searching, supplemented by Google Scholar. Some use was also made of ARROWSMITH [6, 19, 20] and GOPubMed [21], as well as various workflows in the Taverna environment [22-26], including the BioAID_DiseaseDiscovery workflow http:// www.myexperiment.org/workflows/72 written by Marco Roos. Citations and attendant metadata were stored in Endnote (latterly version $\mathrm{X}$ ).

\section{Introduction}

Even under 'normal' conditions, as well as during ischaemia when tissue oxygenation levels are low, the redox poise of the mitochondrial respiratory chain is such that the normally complete four-electron reduction of dioxygen to water is also accompanied by the production, at considerable rates (ca $1-4 \%$ of $\mathrm{O}_{2}$ reduced), of partially reduced forms of dioxygen such as hydrogen peroxide and superoxide (e.g. [27-45]). These 1- and 2-electron reductions of $\mathrm{O}_{2}$ are necessarily exacerbated when the redox poise of the b-type cytochromes is low, for instance when substrate supplies are in excess or when the terminal electron acceptor $\mathrm{O}_{2}$ is abnormally low due to hypoxia or ischaemia. Various other oxygenases, oxidases and peroxidases can also lead directly to the production of such 'reduced' forms of dioxygen in vivo (e.g. [46-48]), with $\mathrm{H}_{2} \mathrm{O}_{2}$ from xanthine oxidase being especially implicated in ischaemia/reperfusion injury (e.g. [47, 49-54]). These molecules (peroxide and superoxide) can cause or contribute to various kinds of oxidative stress. However, this is mainly not in fact because they can react directly with tissue components themselves, since they are comparatively nontoxic, cells have well-known means of dealing with them [55], and they are even used in cellular signalling (e.g. [56-60]). Much more importantly, it is because they can react with other particular species to create far more reactive and damaging products such as hydroxyl radicals, with all these agents nevertheless being known collectively (and indiscriminately) as reactive oxygen species (ROSs). Possibly the commonest means by which such much more damaging species, in particular the hydroxyl radical, are created is by reaction with unliganded or incompletely liganded iron ions [61-63]. The themes of this review are thus (i) that it is this combination of poorly liganded iron species, coupled to the natural production of ROSs, that is especially damaging, (ii) that the role of iron has received far less attention than has the general concept of ROSs, albeit the large literature that we review, and (iii) that this basic combination underpins a great many (and often similar) physiological changes leading to a variety of disease manifestations, and in particular those where the development of the disease is manifestly progressive and degenerative. 


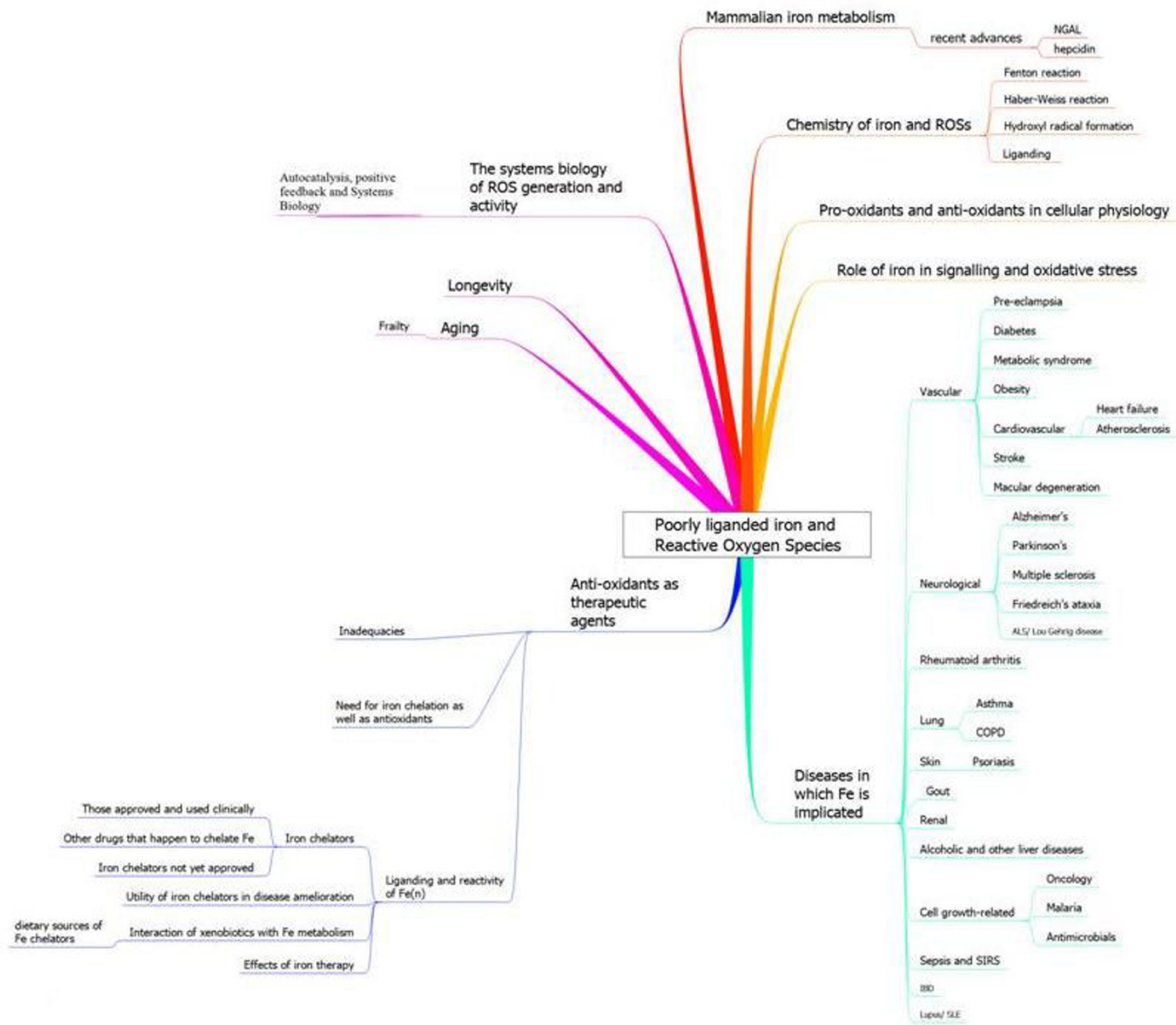

Figure I

An overview of this article, set out in the form of a 'mind map' [64].

An overview of the structure of the review is given in Fig 1, in the form of a 'mind map' [64]. The main literature review for this meta-analysis was completed on June $30^{\text {th }}, 2008$, with some updates being added following the refereeing process.

\section{Some relevant chemistry of iron and reduced forms of oxygen}

While superoxide and peroxide are the proximate forms of incomplete $\mathrm{O}_{2}$ reduction in biology, a reaction catalysed by the enzyme superoxide dismutase [65] serves to equilibrate superoxide and peroxide:

$$
2 \mathrm{O}_{2}^{\bullet-}+2 \mathrm{H}^{+} \rightarrow \mathrm{H}_{2} \mathrm{O}_{2}+\mathrm{O}_{2}
$$

Arguably the most important reaction of hydrogen peroxide with (free or poorly liganded) $\mathrm{Fe}$ (II) is the Fenton reaction [66], leading to the very reactive and damaging hydroxyl radical $\left(\mathrm{OH}^{\bullet}\right)$

$$
\mathrm{Fe}(\mathrm{II})+\mathrm{H}_{2} \mathrm{O}_{2} \rightarrow \mathrm{Fe}(\mathrm{III})+\mathrm{OH}^{-}+\mathrm{OH}^{\bullet}
$$

Superoxide can also react with ferric iron in the HaberWeiss reaction [67] to produce $\mathrm{Fe}(\mathrm{II})$ again, thereby effecting redox cycling:

$$
\mathrm{O}_{2}^{\bullet-}+\mathrm{Fe}(\mathrm{III}) \rightarrow \mathrm{O}_{2}+\mathrm{Fe}(\mathrm{II})
$$


Ascorbate can replace $\mathrm{O}_{2}{ }^{\bullet-}$ within the cell for reducing the $\mathrm{Fe}(\mathrm{III})$ to $\mathrm{Fe}(\mathrm{II})$ [68]. Further reactions, that are not the real focus here, follow from the ability of hydroxyl radicals and indeed $\mathrm{Fe}(\mathrm{n})$ directly to interact with many biological macro- and small molecules, especially including DNA, proteins and unsaturated lipids. Thus [69-73], $\mathrm{Fe}(\mathrm{II})$ and certain $\mathrm{Fe}$ (II) chelates react with lipid hydroperoxides (ROOH), as they do with hydrogen peroxide, splitting the $\mathrm{O}-\mathrm{O}$ bond. This gives $\mathrm{RO}^{\bullet}$, an alkoxyl radical, which can also abstract $\mathrm{H}^{\bullet}$ from polyunsaturated fatty acids and from hydroperoxides. The resulting peroxyl radicals $\mathrm{ROO}^{\circ}$ can continue propagation of lipid peroxidation. Oxidative stress also leads to considerable DNA damage [74-76] and to the polymerisation and denaturation of proteins [77-79] and proteolipids that can together form insoluble structures typically known as lipofucsin (see e.g. [80, 81]) or indeed plaques. Such plaques can also entrap the catalysts of their formation, and thereby point them up. Some of the evidence for these is described below. Many small molecule metabolic markers for this kind of oxidative stress induced by the hydroxyl radical and other 'reactive oxygen species' (ROSs) are known [43, 82-89], and include 8-oxo-guanine [90-94], 8-hydroxy guanine [95], 8-hydroxy-2'-deoxy-guanosine [96, 97], 8-oxo-GTP [98], 4-hydroxy-2-hexenal [99], 4-hydroxynonenal [100], 4-hydroperoxy-2-nonenal, various isoprostanes [101-107], 7-keto-cholesterol [108], many other cholesterol derivatives [109], malondialdehyde [110], neopterin [111], nitrotyrosine [112-115] and thymidine glycol [116, 117]. Note that the trivial names in common use for this kind of metabolite are not helpful and may even be ambiguous or misleading, and it is desirable (e.g. [118]) to refer to such molecules using terminology that relates them either to molecules identified in persistent curated datbases [119] such as ChEBI [120] or KEGG [121], or better to describe them via database-independent encodings such as SMILES [122] or InChI [123-128] strings. (There are other oxidative markers that may be less direct, such as the ratio of 6-keto-prostaglandin $\mathrm{F} 1 \alpha$ to thromboxane B2 [129], but these are not our focus here.)

Overall, it is in fact well established that the interactions between 'iron' sensu lato and partly reduced forms of oxygen can lead to the production of the very damaging hydroxyl radical (e.g. [43, 130-139]), and that this radical in particular probably underpins or mediates many higher-level manifestations of tissue damage, disease, organ failure and ultimately death $[36,137$, 140-143]. While the role of ROSs generally in these processes has been widely discussed, the general recognition of the importance of inadequately liganded iron in each of them has perhaps been less than fully appreciated. One of our tasks here will therefore be to stress this role of 'iron', and to assess the various means of chelating 'iron' such that it does not in fact do this. (Throughout we use 'iron' to refer to forms of $\mathrm{Fe}(\mathrm{n}$, $\mathrm{n}>0$ ) with unspecified ligands, though we absolutely stress that it is the exact speciation and liganding that determines the reactivity of 'iron' in catalysing reactions such as that of hydroxyl radical formation, and indeed its bioavailability generally - inadequate liganding of iron in the required forms can be a cause of anaemia even if the total amount of 'iron' is plentiful.)

For completeness we note the reactions catalysed by superoxide dismutase

$$
2 \mathrm{O}_{2}{ }^{\bullet-}+2 \mathrm{H}^{+} \rightarrow \mathrm{O}_{2}+\mathrm{H}_{2} \mathrm{O}_{2}
$$

and by catalase

$$
\mathrm{H}_{2} \mathrm{O}_{2} \rightarrow \mathrm{H}_{2} \mathrm{O}+1 / 2 \mathrm{O}_{2}
$$

These together, were their activity in the relevant locations sufficiently great, might serve to remove (su) peroxide from cells completely.

In addition to reactive oxygen species there are ions such as the perferryl ion $(\mathrm{Fe}-\mathrm{O})[144]$ and reactive nitrogen species [60, 145-147]. These latter are mainly formed from the natural radical NO, an important inflammatory mediator [148], with peroxynitrite production (from the reaction of NO and superoxide) [46, 149-154] leading to nitrotyrosine [112], or nitro-fatty acid [155, 156] or protein cystein nitrosylation $[157,158]$ being a common means of their detection downstream. Other toxic products of the reactions of $\mathrm{NO}$ include $\mathrm{NO}_{2}, \mathrm{~N}_{2} \mathrm{O}_{3}$, and S-nitrosothiols [159], and the sequelae of some of these may also involve iron [160].

Overall, we recognise that these kinds of inflammatory, oxidative stress-related reactions are accumulative and somewhat irreversible [161], that they are consequently age-related, and (see [162-165] and later), and that most diseases and causes of mortality that are prevalent in the developed world are in this sense largely manifestations of this kind of aging.

\section{Ligands and siderophores}

As well as the reactions described above, ferrous ions will react with oxygen under aerobic conditions to produce ferric ions, and in natural environments there is little to stop this. Consequently, and because these reflect fundamental physicochemical properties of such ions, the problems of both solubility and toxicity were faced by bacteria (and indeed fungi [166-169]) long ago in evolution, and were solved by their creation and 
excretion of (mainly ferric-)iron chelators known as siderophores [170-189] (and for haemophores see [190]). These typically have extremely tight binding constants $\left(K_{\mathrm{f}}>10^{30}[191]\right)$ and can solubilise and sequester iron such that it can be internalised via suitable transporter molecules within the bacterial plasma membrane [192]. Bacterial and fungal siderophores usually form hexadentate octahedral complexes with ferric iron and typically employ hydroxamates, $\alpha$ hydroxycarboxylates and catechols as extremely effective $\mathrm{Fe}^{3+}$ ligands [182]. Since bacterial growth requires iron, it is unsurprising that siderophores are effectively virulence factors (e.g. [174, 193-196]). While upwards of 500 microbial siderophores have been identified [182], with new ones still appearing (some via genomic analyses, e.g. [197]), and with the most common one in medical use, desferrioxamine or DFO, being such a bacterial product (see below), it is an astonishing fact that no human siderophore has been chemically identified, even though such activities were detected nearly 30 years ago [198, 199] (see also [200-205]). As noted by Kaplan [206], "a discovery that mammals produce siderophores would lead to an epochal change in the paradigm of mammalian iron homeostasis." To this end, some recent events have begun to change matters, and our overall knowledge of the regulation of iron metabolism, considerably.

\section{Mammalian iron metabolism}

The total body iron in an adult male is 3000 to $4000 \mathrm{mg}$ and the daily iron requirement for erythropoiesis, the major 'sink', is about $20 \mathrm{mg}$ [207]. However, the loss of iron in a typical adult male is very small $[208,209]$ and can be met by absorbing just $1-2 \mathrm{mg}$ of iron per day $[210,211]$. The careful conservation and recycling of iron - mainly from degrading erythrocytes - is in fact essential because typical human diets contain just enough iron to replace the small losses, although when dietary iron is more abundant, absorption must be (and is) attenuated since higher levels than necessary lead to iron overload and many distressing sequelae contingent on the radical production described above.

A variety of aspects of mammalian iron metabolism have been reviewed in detail elsewhere (e.g. [134, 139, 195, 212-241]), including a series on 'iron imports' [242-248], and for our present purposes (Fig 2) mainly involves the intestinal (mainly duodenal) uptake of $\mathrm{Fe}$ (II) (produced from Fe(III) using a luminal ferrireductase) via a divalent metal ion transporter DMT1/DCT1/ NRAMP $[249,250]$ and its subsequent binding as Fe(III) to transferrin (Tf). The intestinal uptake of haem (heme) occurs via the heme carrier protein-1 (HCP1) [251] and it is thereby internalized, while the iron in heme is

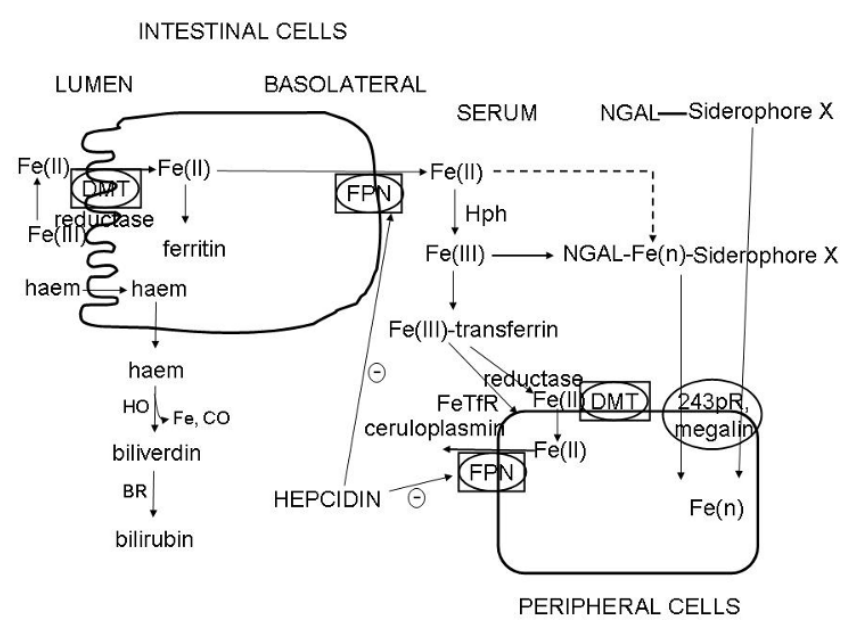

Figure 2

Schematic overview of the main elements considered to participate in mammalian iron metabolism.

liberated by heme oxygenase-1 (HO1) [252-254]. Haem is synthesised in many tissues, especially liver and erythroid cells [255]. Vesicular routes of intestinal transfer may also occur $[256,257]$. Low MW cytoplasmic chelators such as citrate can bind iron fairly weakly and thereby contribute to a labile iron pool (LIP) in the cytoplasm and especially the lysosomes and mitochondria (see [258-262]), while ferritin [263] too can bind cytoplasmic iron (via a chaperone [264]) and is seen as a good overall marker of iron status [265-267]. Iron(II) is subsequently exported through the basolateral membrane of the enterocyte by ferroportin-1 (FPN1) [268-270]. Ferroportin may also contribute to uptake in enterocytes [271]. $\mathrm{Fe}(\mathrm{III})$ may then be produced by hephaestin (Hp) [272] before it is bound by transferrin (Tf), which is the main but not sole means of binding Fe (III) when it is transported through the circulation, with major iron storage taking place in the liver. Similar processes occur in the peripheral tissues, with significant transfer of iron from transferrin occurring via the transferrin receptor [273].

'Free' haem appears in the circulation (it may have a signalling role [274]) and elsewhere largely because of erythrocyte degradation, and it can also greatly amplify the cellular damage caused by ROSs [275], and its degradation pathway via haem oxygenase $[276,277]$ to biliverdin and then using biliverdin reductase to form bilirubin generates 'free' (and potentially redox-active) iron. It would appear, not least because biliverdin has powerful antioxidant properties, that haem oxygenase is more protective than damaging [253, 278-282], even though one of the products of its reaction is Fe that must eventually be liganded (or e.g. incorporated into 
ferritin). (Another product is the gas $\mathrm{CO}$, that has been proposed as a measure of oxidative stress in the lung [283].)

All of the above obviously ignores both some important aspects of the speciation and liganding of iron, as well as the tissue distribution of the specific proteins involved for which latter global information will shortly emerge [284] (http://www.proteinatlas.org/ and see later). It also ignores any discussion on the genetic regulation of iron metabolism (e.g. [285-288]), which is not our main focus.

However, one molecule in particular, hepcidin, has recently emerged as a 'master regulator' of regulation at the physiological level, and we describe some of these new developments.

\section{Hepcidin}

In the liver and elsewhere, many aspects of iron metabolism are regulated by a recently discovered 25amino acid polypeptide called hepcidin [207, 241, 245, $271,289-327]$ that acts in part as a negative regulator of iron efflux [328] by causing the internalisation of ferroportin [329-333]. Hepcidin is produced, partly under the regulation of a receptor called hemojuvelin (e.g. [334]), via an 84-aa precursor called pre-prohepcidin and a 60 mer called pro-hepcidin [304, 335, 336] although the active agent is considered to be the 25 mer referred to above, and with the inactive precursors appearing not to be useful markers [337, 338].

Strikingly, anaemia and anoxia both suppress hepcidin production $[245,339,340]$ (Fig 3), such that just while superoxide production is being enhanced by the anoxia there is more iron being absorbed from the intestine and effluxed into the circulation. In view of the interreactivity of superoxide and iron this could be anticipated to enhance free radical formation, leading to a positive feedback loop in which the problems are amplified: ischaemia/anoxia changes $\mathrm{Fe}(\mathrm{n})$ distribution leading to differential reactivity with the products of anoxia and thus further free radical production. However, hepcidin is overexpressed in inflammatory disease and is an early inflammatory marker [245, 341-345]. Its expression is positively controlled inter alia by SMAD4, and loss of hepatic SMAD4 is thus associated with dramatically decreased expression of hepcidin in liver and increased duodenal expression of a variety of genes involved in intestinal iron absorption, including Dcytb, DMT1 and ferroportin, leading to iron overload [346]. STAT3 is another positive effector of hepcidin expression [347, 348], and ROSs inhibit this effect [349], thereby creating a link between ROSs and Fe metabolism. To

\section{Some effects of Hepcidin}

\section{- Suppressed by hypoxia \\ - Raised by high iron stores and/or inflammation \\ - Hepcidin increase causes internalisation of FPN, with decrease in efflux of $\mathrm{Fe}(\mathrm{II})$ from peripheral cells, causing IL-6-mediated hypoferraemia (but thus more $\mathrm{Fe}(\mathrm{n})$ to remain intracellular) \\ - Lowered hepcidin causes hyperferraemia}

\section{Figure 3}

Some effects of hepcidin, summarizing the fact that hypoxic condition can suppress it and thus lead to hyperferraemia. Since hypoxic conditions can also lead to ROS production the hypoxia-mediated regulation of hepcidin can have especially damaging effects.

understand the exact roles of hepcidin in iron metabolism, it is going to be especially important to understand where it is expressed; fortunately, such studies are beginning to emerge [350].

Overall there is a complex interplay between positive and negative regulation and the organismal distribution of iron caused by changes in hepcidin concentration [351], with in many cases the hypoxic response (decreased hepcidin) seeming to dominate that due to inflammation (increased hepcidin) even when iron levels are high $[352,353]$. Specifically, lowered hepcidin causes hyperferraemia. Hepcidin is also activated by p53 [354], and may play a role in the degradation of atherosclerotic plaques [355]. Another recently discovered protein that is crucially involved in human iron metabolism is NGAL or siderocalin, and while there is some evidence for their co-regulation [356], they have normally been studied separately.

\section{NGAL (also known as lipocalin-2 or siderocalin)}

Lipocalins [357] are a diverse group of ligand-binding proteins that share a conserved structure even in the absence of significant sequence conservation. This core structure includes an eight-stranded anti- parallel $\beta$ barrel that defines a calyx, or cup-shaped structure, enclosing the ligand binding site.

NGAL - neutrophil gelatinase-associated lipocalin - is a $21 \mathrm{kDal}$ glycoprotein first isolated by Kjeldsen and colleagues in 1993 [358]. Synonyms include lipocalin 2, siderocalin, Lcn2, $\alpha 2$-microglobulin-related protein or neu-related lipocalin (in rats) $[359,360]$ and (in mice) 
24 p3 or uterocalin [361]. Although lipocalins are well known to be involved in the sequestration and transport of a variety of ligands, the natural ligand of NGAL (as is the case with many lipocalins) was not initially known even in terms of its chemical class. This changed with the seminal paper of Goetz and colleagues [362] (and see [206]) who purified recombinant NGAL from a particular strain of $E$. coli and found that its structure contained a negatively charged ferric siderophore with a subnanomolar dissociation constant that it had extracted from its bacterial host, and that the apo form of this molecule could also act as a potent bacteriostatic agent by sequestering iron (see also [363-367]). A companion paper [368] showed that the iron-delivering activity was expressed in mammalian cells. The structure of NGAL is now known [369] and one of its interaction partners is a matrix metalloproteinase [370] to which it can presumably donate a metal ion and in the complex decrease its degradation [371].

The finding that NGAL was one of the most highly expressed proteins following ischaemia-reperfusion injury in kidney cells [372-374], and prognostic of kidney damage long before the more traditional marker creatinine was raised significantly, has led to considerable interest in this protein, especially as a marker of renal injury [375-389], and perhaps as a therapeutic [375]. Devireddy and colleagues [390] identified a receptor that internalizes $24 \mathrm{p} 3$, and internalization of iron bound to $24 \mathrm{p} 3$ prevents apoptosis. In contrast, internalization of the apo form of $24 \mathrm{p} 3$ that does not contain iron led to cellular iron efflux and apoptosis via the proapoptotic protein Bim [391]. In humans the megalin receptor can bind siderocalin (and its siderophore payload) and mediate its intracellular uptake [392]. Oxidative stress can also induce its expression [393], and it is protective against it [394].

Exogenously administered NGAL also markedly upregulates heme oxygenase-1, a proven multifunctional protective agent in experimental Acute Kidney Injury (AKI) that is thought to work by limiting iron uptake, promoting intracellular iron release, enhancing production of antioxidants such as biliverdin and carbon monoxide, and inducing the cell cycle regulatory protein p21 [279, 395, 396]. Because of this multifaceted protective action, NGAL has emerged as a prime therapeutic target in ischaemic AKI [379].

Structural and direct binding studies have suggested that siderocalin tends (although not exclusively) to bind catecholate-type ligands, rather than hydroxamate- or carboxylate-based siderophores, at least when tested with microbially derived siderophores [362, 363, 365] (but cf. [369] for claims, disputed [360] and not now accepted, as to the binding of bacterially derived formyl peptides!). The role of NGAL, as a siderophore-binding agent, is thus consistent with the widespread recognition that ironinduced radical generation is intimately involved in a variety of renal and other diseases [397, 398]. However, while it is certainly the case that siderocalin can reduce the virulence of bacteria when it binds the relevant bacterial siderophores [362-367] and that bacteria can 'evade' this by synthesising siderophores that siderocalin cannot bind (e.g. $[186,187,399-401])$, it is questionable whether the only role of siderocalin lies in fact in its antibacterial activity. Rather we would suggest that its main role is in sequestrating iron via a human siderophore to stop inappropriately liganded iron from producing damaging oxygen radicals. Consistent with this iron-liganding role for human biology is the fact that the tissue most highly expressing NGAL under normal conditions is bone marrow [360, 402], the site of erythropoiesis. The liganding can be extensive; as Goetz and colleagues [362] note, "During inflammation, concentrations of NGAL can increase to levels, with concentrations approaching 20-30 $\mathrm{nM}$ in the serum [403], presumably adequate to bind all available iron as ferric siderophore complexes".

Significant changes in NGAL expression have also been observed, for instance, during kinase-mediated signalling [404, 405], in cardiovascular disease [406-409] and in cancer [410-412].

These findings on the kidney and the role of NGAL, together with the important knowledge that its chief ligand is probably an unknown human siderophore (Figs 2, 4), thus lead us to consider the role of this

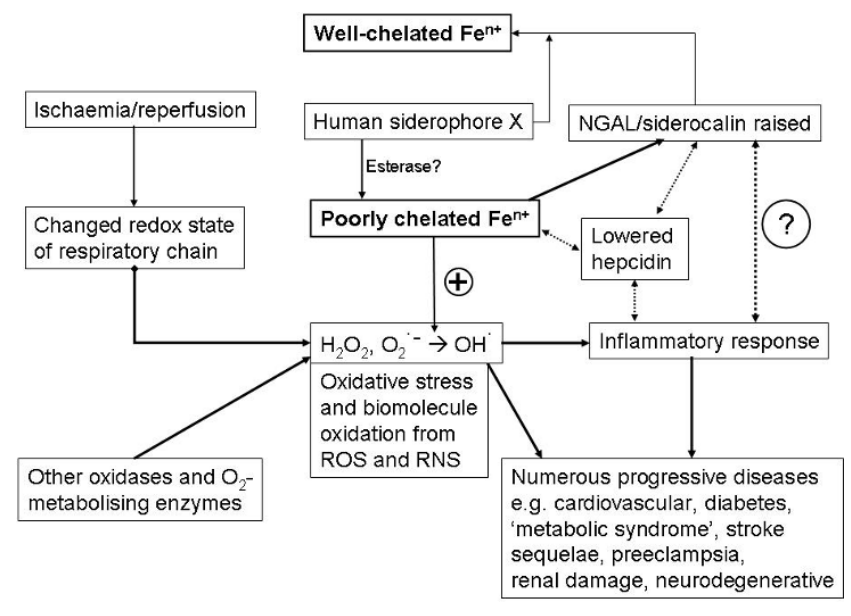

Figure 4

Overview of the roles of ischaemia, ROSs, poorly liganded iron and the iron metabolism regulators HGAL and hepicidin in effecting inflammation as a physiological level. 
system (and unliganded iron generally) in a whole series of other diseases that all share many characteristics of oxidative stress and inflammation (see also [413]). A similar thesis, albeit with comparatively little stress on iron, is the leitmotif of Finch's recent detailed monograph [163]. The theme of these sections is thus to stress the fact that while the role of ROSs in general in such syndromes has been pointed up previously, that of iron as a major culprit has not so generally yet been stressed, notwithstanding that there is in fact a great deal of pertinent literature that we here highlight as the focus of this review.

\section{Some disease manifestations in which iron may be implicated \\ Preeclampsia (PE)}

Another important disease that shares many of the same properties (or at least sequelae) of renal impairment, and may have the same fundamental aetiology, is preeclampsia. This is the most significant cause of morbidity and mortality in pregnant women [414]. The chief clinical manifestations at time of diagnosis are a raised blood pressure (hypertension) [415] and proteinuria, together with raised creatinine, consistent with the reversible existence (since it is relieved upon delivery of the baby) of renal impairment. However, prognostic markers that might manifest early in pregnancy are lacking, and would be highly desirable. There is widespread agreement [416] that a poor connection of the placenta to the uterus leads to ischaemia and thus oxidative stress, with a substantial involvement of apoptosis during the placental remodelling [417-423]. Since preeclampsia-like syndromes can be induced in pregnant animals by surgical restriction of the uteroplacental blood supply [424], it is presumed that bloodborne agents arising from the ischaemic placenta are the cause of the generalized endothelial cell damage and inflammatory responses that give rise to the symptoms of hypertension, proteinuria, and sudden oedema characteristic of preeclampsia [70]. Indeed, many studies implicate oxidative stress as a substantial contributor to this [425-489], while some have noted the importance of iron status $[70,133,450,490-511]$, and so far as is known the transporters of iron in the placenta are similar to those in other cells [512]. Oxidative stress of this type is of course inflammatory in nature and inflammation is observed in PE [472, 476, 484, 486, 513-519]. We suggest strongly that it is the combination of inadequately liganded $\mathrm{Fe}$ (II or III) and superoxide/peroxide leading to $\mathrm{OH}^{\bullet}$ formation that is the chief mechanistic cause of the downstream events that manifest in $\mathrm{PE}$, and that appropriate removal by liganding/chelation or otherwise of these ions would prove of therapeutic benefit. (Iron status has also been implicated in other pregnancy and neonatal disorders [520-524].) There is evidence too for the involvement of the radical NO $[456,525]$.

We note that it is quite common nevertheless for iron to be prescribed during pregnancy, especially during its latter stages $[526,527]$, and that this does of course lead to oxidative stress $[528,529]$.

Oxidative stress is caused both by the initial rate of production of superoxide and the rate of their conversion into $\mathrm{OH}^{\bullet}$ radicals. The former can be induced by hypoxic conditions such as occur at high altitude, and one prediction, that is borne out $[487,530]$, is that $\mathrm{PE}$ should therefore be more prevalent at high altitude. Erythropoietin may be a marker for oxidative stress in pre-eclampsia [531].

Regarding the second stage, predictions include that PE should be more common in those suffering from diseases of iron metabolism. Although such mothers are of course less well $a$ priori, this prediction is borne out for $\alpha$-thalassemia $[532,533]$ although not, interestingly, for haemochromatosis [534]. We note in this context that thalassaemia not only predisposes towards PE but is known in general to cause hepcidin to decrease and NGAL to increase [352, 353, 356, 535], with consequent and inevitable iron dysregulation.

Another prediction is then that hepcidin should be changed in pre-eclampsia. Although no serum measurements have been reported to date, it is of extreme interest that - while they took it to be an antimicrobial peptide rather than an iron regulator - a recent study by Knox and colleagues of placental gene expression in a mouse model of PE showed that hepcidin expression increased by a greater factor than that of any other gene save one [536], consistent with the view that major changes in the regulation of iron liganding and metabolism underpin PE.

Finally, we note that NGAL is significantly implicated in pregnancy, and was even named uterocalin in mice to reflect its high expression in the uterus [361, 537-539]. A very recent study [540] suggests that it may be a useful second trimester biomarker for pre-eclampsia.

\section{Diabetes}

Type 2 diabetes and insulin resistance are known complications of pregnancy (e.g. [541-545]), and also predispose towards PE. In a similar vein, various types of pregnancy-related intrauterine growth restriction predispose towards diabetes in later life [546, 547], pointing up the progressive nature of these syndromes. Metabolic biomarkers for the one can thus be predictive of the other [548], consistent with a common cause. Certainly 
ROSs are known to play a substantive role in both insulin resistance [549-556] and in a variety of diabetic sequelae [95, 557-559], and mitochondrial dysfunction may be an early step in this [560]. Some anti-diabetic drugs, such as the 'glitazones' that are considered to act on Peroxisome Proliferator Activated Receptor (PPAR) $\gamma$, may also act by decreasing ROS production (e.g. [561-565]), and are even active aganst cerebral ischaemia and stroke [566-569]. As with most if not all of the other diseases we review here, studies of pro-inflammatory markers (such as TNF- $\alpha$, IL- 1 and C-reactive protein [570]) during the development of diabetes show its aetiology to be inflammatory in nature [553, 571-590]. Iron 'excess' is also a known feature of gestational diabetes [591-593], and is a clear risk factor for the disease even in 'normal' populations [594-603], and indeed diabetes is a classical consequence of iron overloading as seen in hereditary haemochromatosis [604]. Serum ferritin and body iron stores are strongly associated with diabetes [603, 605-609], including prospectively [610], while changes in visfatin are also intimately involved in changes of iron metabolism (with pro-hepcidin being elevated) [611]. Most importantly, lipocalin 2 (siderocalin/NGAL) is strongly associated with the development of diabetes [612, 613]. Lowering iron improves insulin sensitivity [598, 614], and metallothionein is also protective [615-619]. There seems little doubt that iron status is a major determinant of the development of type 2 diabetes [620].

Non-transferrin-bound iron is also considerably elevated in type 2 diabetes [621], and this too is exacerbated by vitamin C. Iron metabolism is substantially deranged in type 2 diabetes and the metabolism of glucose (a reducing sugar) interacts significantly with iron metabolism [598]. Iron is also strongly implicated in nonalcoholic steatohepatitis, considered an early marker of insulin resistance [622-624]. Well-known diabetic complications include retinopathies, and it is noteworthy that elevated levels of ferritin can lead to cataract formation $[625,626]$.

\section{The metabolic syndrome}

Although some of its origins may be pre-natal [547], many of the features of these diseases are also seen in the (so-called) Metabolic Syndrome [627-631]. Thus, serum ferritin is also related to insulin resistance [606, 632, $633]$ and iron levels are raised [624, 634, 635]. Of course diabetes and the Metabolic Syndrome are also closely coupled, so it is reasonable that features observed in the one may be observed during the development of the other. The metabolic syndrome is also an independent indicator for chronic kidney disease [636] and may be related to liver steatosis [637]. Metabolic disorders of this type too are closely intertwined with inflammation $[575,581,587,638]$, that is of course stimulated by ROSs whose generation is increased by high-fat diets [639]. Thus, our role here is to point up the existence of a considerable body of more-than-circumstantial evidence that here too the progressive and damaging nature of these diseases may be caused, in part, by inappropriately chelated iron.

\section{Obesity}

"As previously pointed out by Booth et al. [640], 100\% of the increase in the prevalence of Type 2 diabetes and obesity in the United States during the latter half of the 20th century must be attributed to a changing environment interacting with genes, because $0 \%$ of the human genome has changed during this time period." [629]

It is well known that there has been a staggering increase in the prevalence of obesity, diabetes, and especially type 2 diabetes, in the last 50 years or so, and that this increase is expected to continue (e.g. [641-643] and http://www.who. int/diabetes/). Equally, it is now well known that obesity, metabolic syndrome, diabetes and cardiovascular diseases are all more or less related to each other [643], and the question arises here as to whether dysfunctional iron metabolism might be a feature of each of them. In the case of obesity per se, however, we see no major evidence as yet for a causative role of deranged iron metabolism or chelation in causing obesity. Indeed, while they are related [644], what little evidence there is $[645,646]$ suggests that the converse may be true, i.e. that changes in iron metabolism might be consequent upon obesity (possibly via peroxide generation [639]). Importantly, considerable evidence suggests that obesity and inflammation are significantly related [163, 486, 575, 581, 642, 647-664], not least because adipocytes produce and release various adipokines including pro-inflammatory cytokines such as IL-6 and TNF- $\alpha[575,649,650,665-671]$. It is likely that it is the combination of overfeeding-induced obesity and inflammation (partly induced by the obesity itself [672]) that leads to diabetes [673]. Certainly there is evidence for increased ROS production in obese mice, possibly mediated in part via the fatty acid-induced activation of NAPH oxidase [674], while obesity is linked $[675,676]$ to urinary levels of 8-epi-PGF $2 \alpha$, a well established marker of oxidative stress (qv). Fig 5 summarises the above in a manner that stresses the roles of iron, overfeeding and inflammation in the genesis of these processes, and notes that interference in several of these steps is likely to be required to limit their progression to best advantage.

\section{Hypertension}

As well its significance in pre-eclampsia (see above), hypertension is a well known risk factor for many 


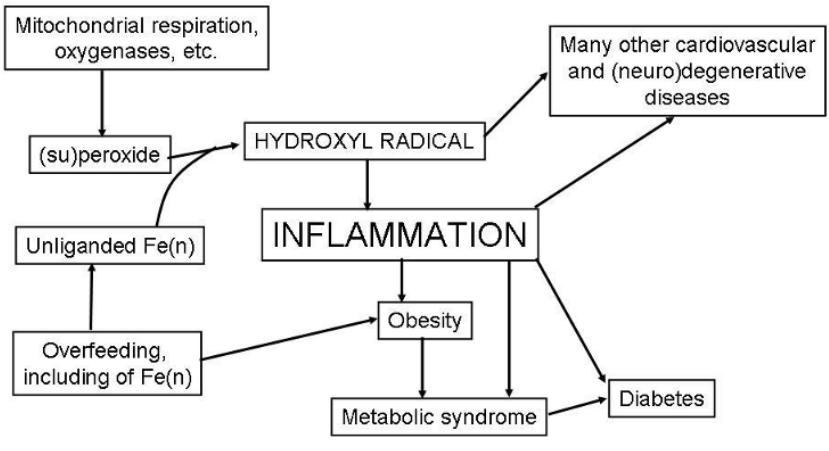

Figure 5

Role of inflammation caused by hydroxyl radical formation in the interactive development of obesity, the metabolic syndrome and diabetes. Intervention at multiple steps is likely to be most beneficial in alleviating this kind of progression.

cardiovascular and related disease (e.g. [677]), and there is considerable evidence that its underlying cause is inflammatory in nature [678-686], is related to the metabolic syndrome and obesity (e.g. [648, 687-691]), and may be mediated mainly via ROSs [692]. There is evidence that some of its sequelae may be mediated via iron [693, 694].

\section{Cardiovascular diseases}

It is well known that elevated iron stores can predispose to coronary artery disease and thence myocardial infarction. The 'iron hypothesis' of the benefits of some iron depletion due to menstruation was devised to account for the lowering of heart-disease risk in young women (that disappears in those post-menopause) and was proposed by Jerome Sullivan in 1981 [695-698] (and see also [699, 700]). (In this sense, the lack of menstruation during pregnancy would predispose to a comparative abundance of iron, as is indeed found - see above.) It is of particular interest that the well-known adverse vascular effects of homocysteine (in inhibiting flow-mediated dilatation) are in fact iron-dependent [701-703], and that reducing homocysteine (e.g. by folate supplementation) in the absence of lowering iron has shown no clinical benefit to date [704], thereby suggestion iron mediation. By contrast, iron stores represent an established risk factor for cardiovascular disease [705].

Of course many factors such as lipid levels, stress, smoking and so are well-known risk factors for cardiovascular, coronary artery disease and related diseases. Indeed kidney disease is well established as a risk factor for cardiovascular disease [706-708] (and indeed stroke [709]), all consistent with their having in part a common cause - we believe inflammation). Our purpose here, within the spirit of this review, is to indicate the evidence for the involvement of inappropriately chelated iron in cardiovascular diseases too. There is no doubt that the iron-mediated causal chain of ischaemia $\rightarrow$ (su)peroxide $\rightarrow \mathrm{OH}^{\bullet}$ radical formation occurs during the development of heart disease, especially during reperfusion injury [710-713], and suitable iron chelators inhibit this [714, 715] (see also [716, 717], and for thalassaemia [718]). Iron is also involved in the protection that can be produced by ischaemic preconditioning [719, 720]. Erythropoietin, a hormone with multiple effects that may involve iron metabolism, is also protective [721, 722].

\section{Heart failure}

The sequelae of heart failure are complex, and involve a chronic and continuing worsening of a variety of physiological properties. ROSs are certainly involved here, since allopurinol (a potent inhibitor of xanthine oxidase) improves prognosis considerably [723], and uric acid is a well known biomarker for heart failure (see e.g. [724, 725]). Biopyrrins, degradation products of bilirubin and thus markers of oxidative stress are also considerably increased [726]. Anaemia is a common occurrence (and risk factor) in heart failure [727-729], again implying a role for dysregulated iron metabolism and a need to understand the exact speciation of iron in chronic anaemias linked to inflammatory diseases [730].

It is next on the formation of atherosclerotic plaques that our attention is here focussed.

\section{Atherosclerosis}

Atherosclerosis is a progressive inflammatory disease [731-762] characterized by the accumulation of both oxidised lipids and various fibrous elements in arteries, often as plaques [763, 764]. Iron and oxidised lipids are both found in atherosclerotic lesions [141, 765-777], and iron depletion by dietary or other means delays this [778-782]. There is a correlation between iron status and atherosclerosis [766, 776, 783-794], evidently caused in part by the known ability of poorly liganded iron to effect lipid $[765,784]$ and protein peroxidation, and by the effects of primed neutrophils [795] and transferrin [758]. In this context, exogenous ferric iron is deleterious to endothelial function [796], while iron chelation improves it [797-800]. However, phlebotomy provided no clinical benefit here [801]. Note that iron levels in plaques correlate with the amount of oxidised proteins therein [771], and that in one study [767], the EPRdetectable iron (essentially $\mathrm{Fe}(\mathrm{III})$ ) in atherosclerotic tissue was seventeen times greater than that in the equivalent healthy tissue; this is not a small effect. (Iron, 
as part of the general ROS cascade, has also been implicated in gallstone formation, where melatonin has proved protective $[802,803]$.)

Statins, typically developed on the basis of their ability to inhibit the enzyme HMG-CoA redutase and thus decrease serum cholesterol, are well established to have benefits in terms of decreasing the adverse events of various types of cardiovascular disease [804], albeit that in many populations (e.g. [805-807]) cholesterol alone is a poor predictor of cardiovascular disease, especially in the normal range. However, a known target of statins different from HMGCoA reductase is the $\beta_{2}$-integrin leukocyte function antigen-1 (LFA-1) $[808,809]$ and in this context, it is important to note that the clinical benefits of the statins are certainly not due solely to their cholesterol-lowering ability via the inhibition of HMGCoA reductase (see e.g. [163, 281, 650, 807, 808, 810-840]), and different statins can cause a variety of distinct expression profiles [841] that are inconsistent with a unitary mode of action. The apparent paradox [842] that lipid-lowering statins do indeed exhibit epidemiological disease-lowering benefit, while having little effect on plaques, is arguably well explained, especially within the context of the present review, via their additional anti-inflammatory effects $[817,819$, 843-863, 163, 807, 823-825, 830, 836, 837, 864-892], acting upstream of the nuclear transcription factor NF- $\kappa \mathrm{B}$ (and see later). It is also extremely relevant, for instance, that some statins have metal chelating properties [893].

It has been pointed out that many measures of iron stress are inappropriate, since it is only the redox-active form of iron that is likely involved in oxidative stress. Serum ferritin is considered by some to be the most reliable marker of iron status in general [894], although it is not well correlated with iron distribution in the heart [895], for instance. What is clear, however, from the above is that deranged iron metabolism is intimately and causally involved in the formation of atherosclerotic lesions, and that appropriate iron chelation can help both to prevent and to reverse this.

Iron status is also closely involved in other chronic vascular diseases, and in the behaviour of wounds [896-899].

\section{Stroke}

Stroke is caused by ischaemia, leading to inflammation [900] and to the formation of ROS and other damaging free radicals [901] in the brain (which is high in metal ions [902]), and is exacerbated by existing inflammation see e.g. $[903,904]$. Thus, another prediction is that iron excess should also aggravate the sequelae of stroke, and that appropriate chelation or free radical trapping agents should mitigate these effects. These predictions are indeed borne out [138, 905-914]. It is also of considerable interest that plasma NGAL levels are increased in stroke [406]; it is noteworthy that this can be seen in plasma despite the localised origin of the disease.

A variety of other studies have shown the beneficial treatments in stroke models of anti-inflammatory and antioxidant treatment, i.e. treatments that lower the amount of ROSs (e.g. [915-922]), as well as of preconditioning [923]. Given its role in iron metabolism, it is of considerable interest that erythropoietin also seems to be very effective in protecting against brain ischaemia/reperfusion injury and stroke [924-939], by a mechanism independent of erythropoiesis [940-942], and one that appears to involve anti-inflammatory activity [943].

\section{Alzheimer's, Parkinson's and other major neurodegenerative diseases}

Oxidative stress and inflammation are early events of neurodegenerative diseases [920, 944-957] such as Alzheimer's disease (e.g. [958-973]), where plaque formation precedes neurodegeneration [974]. Iron (and in some cases copper) is also strongly implicated in a variety of neurodegenerative diseases [944, 981, 945, 958, 982-985, 962, 986-989, 950, 990-1021, 917, 1022-1034, 278, 1035-1055, 43, 1056-1059, 285, 1060, 972, 1061-1065, 141, 975, 1066-1087].

Indeed Thompson and colleagues comment [136] that "The underlying pathogenic event in oxidative stress is cellular iron mismanagement" and stress that "Multiple lines of evidence implicate redox-active transition metals, such as iron and copper, as mediators of oxidative stress and ROS production in neurodegenerative diseases". There is also ample evidence for its presence in the plaques characteristic of Alzheimer's disease [1004, 1013, 1027, 1088], just as in those of atherosclerosis (see above). Note too that iron can catalyse the oxidation of dopamine to a quinine form that can bind covalently to and then aggregate proteins [1089]. Kostoff [1090] has used a very interesting literature-based discovery approach to highlight the role of oxidative stress in the development of Parkinson's disease.

Other papers highlight the role of iron in multiple sclerosis [899, 991, 1091-1098] and in prion diseases $[967,1099,1100]$. However, a particularly clear example of iron-mediated neurodegeneration is given by the sequelae consequent upon lesions in a protein known as frataxin involved in the disease Friedreich's ataxia (FA). 


\section{Friedreich's ataxia}

A number of repiratory chain components contain nonheme iron, and the question arises as to how they acquire it [1101]. Frataxin is a mitochondrial iron chaperone protein [1102-1109], involved in the safe insertion of $\mathrm{Fe}(\mathrm{II})$ during the production of $\mathrm{Fe}-\mathrm{S}$ centres in the mitochondrial respiratory chain [1110]. As are some other aspects of iron metabolism [1111], it is highly conserved in eukaryotes from yeast to humans [1112, 1113], a fact that made the unravelling of its function considerably easier [1114-1123]. Friedreich's ataxia (FA) is a neurodegenerative disorder that arises from a genetic deficit of frataxin activity, whether by a missense mutation or, much more commonly, via the addition of GAA trinucleotide repeats [1107, 1124-1127]. As well as the neurodegeneration and measurable iron deposition, clinical symptoms include cardiac hypertrophy [1128] and (pre-)diabetes [1129], consistent with the general thesis described here that all are in part manifestations of iron dysregulation, and in which suitable chelation may be beneficial [1130, 1131] (but cf. [1132]).

ROSs are undoubtedly involved in FA [1133, 1134], specifically via Fenton chemistry [1135, 1136], since the attenuation of $\mathrm{H}_{2} \mathrm{O}_{2}$ production (but not of superoxide) [1110] ameliorates the disease [1137]. The deficit in frataxin causes both an increase in $\operatorname{ROS}\left(\mathrm{H}_{2} \mathrm{O}_{2}\right)$ production via the mitochondrial electron transport deficiency [1138] as well as a dysregulation in iron metabolism, potentially a very damaging synergistic combination (see later). Elements of its (in)activity that are seen as paradoxical [1139] are in fact easily explained when one recognises that it is the combination of free iron with $\mathrm{H}_{2} \mathrm{O}_{2}$ that is especially damaging. The neonatally lethal GRACILE syndrome is also caused by a failure of iron chaperoning into mitochondrial complex III due to mutations in the BCS1L gene [1140, 1141].

\section{Amyotrophic lateral sclerosis (ALS) or Lou Gehrig's disease}

ALS is another progressive inflammatory [1142] disease in which motor neuron death causes irreversible wasting of skeletal muscles. It has largely defied efforts to uncover the genetic basis of any predisposition [1143], save for a very clear association with defects in a $\mathrm{Cu} / \mathrm{Zn}$ superoxide dismutase [33, 1144-1146] that can obviously lead to an increase in the steady-state levels of superoxide (and hence hydroxyl radical formation). There is also significant evidence for the involvement of iron [1147, 1148]. Drug therapies have to date shown rather limited benefits, and more in mouse models of $\mathrm{Cu} / \mathrm{Zn}$ SOD deficiencies than in humans, though iron chelation therapy does not seem to have figured heavily, and it is recognised that combination therapies might offer better prognoses [1149].

\section{Aging}

Aging or senescence is defined as a decline in performance and fitness with advancing age [1150]. Iron stores tend to increase with age [1151-1155], partly due to dietary reasons [1156] (and see [1157, 1158]), as does anaemia [1159, 1160]. So too does the expression of NGAL/Lcn2/siderocalin, a process that can be reversed by melatonin [1161]. Mainstream theories of aging [163, $165,1162-1180$ ] recognise the relationship between progressive inflammation, cellular damage and repair and the higher-level manifestations of the aging process, and ('the free radical theory of aging' [1163, 1181]) ROSs are of course strongly implicated as partial contributors to the aging process (e.g. [31, 43, 980, $1163,1182-1206])$. Needless to say, not least because of the low steady-state net rate of generation of the various ROSs [1207], few studies have managed to be very specific mechanistically [1208], but it should be clear that all ROSs are not created equal and we need here to concentrate mainly on the 'nasty' parts of ROS metabolism, and in particular on the hydroxyl radical as generated via poorly liganded iron and on peroxynitrite, and to have the greatest effects we need to inhibit both their generation and their reactivity (see Systems Biology section, below). The iron content of cells also increases as cells age normally [1209]. As many diseases increase with age, probably via mechanisms highlighted herein, treating aging can thereby treat disease [162], and it is important to recognise that most 'diseases' are in fact consequences of aging (despite the considerably greater historical focus on the former).

\section{Frailty}

One issue of aging is not that just it happens but that it can manifest in a series of essentially undesirable physiological changes, referred to as frailty [1210], in which ROSs have also been strongly implicated. Indeed, there are many parts of physiology and metabolism that lose functionality during aging (e.g. the cardiovascular system [1211] and of course cognitive function [1212]), and iron metabolism is known to change considerably as humans age [980, 1213], with anaemia a typical accompaniment of aging [1214]. The question then arises as to how much of this deranged iron metabolism is causal in accelerated aging, and this is not easy to state at this time. However, lowering iron does increase the lifespan of Drosophila [1215] and yeast [1105]. At all events, the purpose of this rather brief section is to point out to researchers in aging, frailty and gerontology generally the relevance of inadequately controlled iron 
metabolism as a major part of ROS-induced injuries that may accelerate the aging process.

\section{Longevity}

Although aging and longevity are not of course the same thing, and longevity is not a 'disease', studies of aging are often performed with the intention of improving our understanding of longevity [1216], and certainly longevity is linked to age-related disease [1217]. However, the longevity of many organisms can be varied by the manipulation of any number of diseases or processes. This said, caloric or dietary restriction is a well-known contributor to longevity (indeed the only reliable one in pretty well all species [1218-1223] - although possibly not $H$. sapiens [1180]), and appears to act at the root of the processes involved [1224]. Caloric restriction appears to be associated with a considerably lowered rate of production of ROSs and accrual of ROS-induced damage $[1207,1225-1228]$, and this is to be expected on general grounds in that a restriction of substrate supply will make the redox poise of mitochondria [1229] more oxiding and thereby minimise the amount of 1- and 2electron reductions of $\mathrm{O}_{2}$ to form peroxide and superoxide. It is therefore also of great interest that caloric restriction also benefits iron status [1230] and that it is this improved iron status that in part promotes longevity [1231]. Caloric restriction has also been shown to effect a differential stress response between normal and tumour cells [1232], although this study did not look at relative iron status.

Antioxidants can also influence lifespan. Thus (rather high doses of) melatonin extended the lifespan (and stress resistance) of Drosophila [1191, 1233-1235] while that of Caenorhadbitis elegans could be extended by mimics [1236] of SOD and catalase [1187], and by a variety of antioxidant and other pharmacological agents [1237].

As an example, let us consider C. elegans. Mutants with a decreased activity of the insulin-like growth factor signalling pathway (e.g. daf2 mutants that have a greater amount of the DAF16 FOXO-like transcription factor) can live for nearly twice as long as wild types [1238, 1239] and produce more catalase, superoxide dismutase (sod-3) [1240] and glutathione-S-transferase.

Overall, it is the potent combination of oxidative stress, already leading to damaging peroxides and radicals, and its catalysis and further reactions caused by inappropriately chelated iron, that causes a 'double whammy'. Indeed, iron, copper and $\mathrm{H}_{2} \mathrm{O}_{2}$ have been referred to as the 'toxic triad' [1032]. While there is comparatively little that we can do about the production of superoxide and peroxide, we can (by pharmacological or dietary means) try and improve the speciation of iron ions.

\section{Rheumatoid arthritis}

One disease whose aetiology is well known to be bound up with ROSs is rheumatoid arthritis (RA) [36, 1241-1246]. What is known of the role of iron metabolism? Generally an overall low iron status anaemia - is a characteristic of rheumatoid arthritis [1247-1251], whereas by contrast iron is elevated in the synovial fluid of arthritic joints [1252-1255]. This suggests a significant derangement of iron metabolism in RA as well, and a mechanism [1256-1259] in which elevated superoxide liberates free iron from ferritin in synovial fluid (and elsewhere [1260]), thereby catalysing further the damaging production of hydroxyl radicals. This autocatalytic process (Fig 6) is, even in principle, especially destructive (and may account for species differences in sensitivity to iron loading [1261]). Note that erythrocytes when oxidized can also release free iron [524]. Natural antioxidants such as vitamin E are also lowered [1262]. There is some evidence that appropriate iron chelators can ameliorate the symptoms of RA [1263], though membrane-impermeant chelators such as desferroxamine cannot [1264].

One interesting feature of RA is that in $75 \%$ of women it is strongly ameliorated during pregnancy [1265-1267]; although the multifactorial nature of this observation has made a mechanistic interpretation difficult, from everything that we have seen so far it would be surprising if changes in iron metabolism were not strongly involved.

An interesting related feature is the 'restless legs syndrome' [1268-1270], that is often associated with

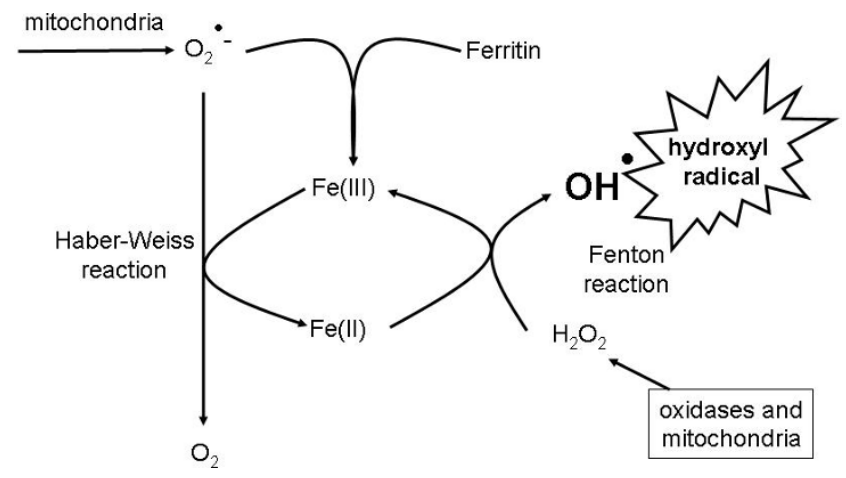

Figure 6

Catalysis and autocatalysis in the Haber-Weiss and Fenton reactions leading to the production of the hydroxyl radical, including the liberation by superoxide of free iron from ferritin. 
iron deficiency and pregnancy. Serum transferrin receptor seems to be a rather sensitive measure of this iron deficiency [273, 1271, 1272]. There are relationships with other syndromes that we discuss here, such as cardiovascular disease [1273], but in the present review it is of especial interest that a dysregulation of iron metabolism appears to play a significant role [1274]

\section{Lupus (Systemic Lupus Erythematosus)}

Lupus, or Systemic Lupus Erythematosus (SLE) [1275, 1276] describes a syndrome, somewhat related to arthritis and rheumatism, of broadly auto-immunological or inflammatory origin, and with a large variety of manifestations (e.g. [1277-1285]), but characterised in particular by fatigue [1286], often as a result of anaemia. This of course points to a certain level of iron dysregulation (of any number of causes), and there is certain some evidence for this [1274, 1287]. Thus, while anaemia is a feature of the disease, serum ferritin may be raised in SLE [1288], and some of the usual lipid markers of oxidative stress (that can be a result of hydroxyl radical production catalysed by poorly liganded iron) are also present [1289].

There is also a very interesting linkage between SLE and vitamin D metabolism [1290], something that has also come up in relation to the statins and atherosclerosis ([832, 1291], but cf. [1292]), and indeed it there is evidence that statins may be of benefit in the treatment and even reversal of lupus [1293, 1294]. Indeed, from a much more general point of view, there is precedent for these kinds of linkages being used to uncover unknown mediators in disease states that may be worth pursuing (e.g. $[6,19,20,1295,1296])$.

\section{Asthma}

Asthma is a well-known inflammatory disease, and has been linked with ROS generation [1297] catalysed by iron [276, 1298-1300].

\section{Inflammatory bowel diseases (IBD)}

By definition, IBD such as Crohn's disease and ulcerative colitis are inflammatory diseases, and while the inflammation and ROS production are well established here, their origins are somewhat uncertain [1301-1304]. They are frequently accompanied by anaemia, implying a derangement in iron absorption and/or metabolism [1305, 1306], and very probably absorption [1307, 1308]. The anaemia may be monitored by ferritin and transferrin receptor levels, and its correction is possible by iron supplementation plus erythropoietin [1309-1315]. One may suppose that some of the issues here relate to iron speciation, that is usually not measured in these studies.

\section{Age-related macular degeneration}

Age-related macular degeneration (AMD) [1316] is now the leading cause of blindness and visual disability in the elderly in developed countries [1317-1320]. Many components of atherosclerotic plaques have also been demonstrated in drusen [1321], a characteristic of AMD and, as here, it is reasonable to propose a common mechanism of pathogenesis between AMD and atherosclerosis. Retinal iron levels increase with age [1322], iron is significantly implicated in AMD [1323-1329], and iron chelation may help to reverse the process [1330]. Dietary antioxidants are also protective [1331]. The source of the iron appears to be excess angiogenesis and leakage from blood vessels catalysed by VEGF, and a PEGylated aptamer [1332-1334] against VEGF (pegaptanib) or a monoclonal antibody (ranibizumab) have shown significant promise in the treatment of macular degeneration [1335-1340]. Plausibly a combination therapy with one of these plus a suitable iron chelator might be even more effective.

\section{Psoriasis}

Psoriasis is an anflammatory disease in which the production of free radicals and ROSs are strongly implicated [1341-1343]. Here too there is clear evidence for the involvement of a deranged iron metabolism [1343-1345]. Early attempts at therapy with a series of unusual iron chelators (that unfortunately had side effects) [1346] do not seem to have been followed up.

\section{Gout}

Gout is another important inflammatory disease, characterised by the accumulation of uric acid [1347]. There is considerable evidence that this too is a disease of iron overload, and that uric acid accumulation - as both an antioxidant and an iron chelator [1348] - is in response to the iron overload [1349, 1350] and with highly beneficial remission of gouty symptoms occurring on depletion of iron by phlebotomy [1351].

\section{Alcoholic and other forms of liver disease}

It is known that with chronic excess, either iron or alcohol alone may individually injure the liver and other organs, and that in combination, each exaggerates the adverse effects of the other. Specifically, in alcoholic liver disease, both iron and alcohol contribute to the production of hepatic fibrosis [1352-1358]. Iron overload is well known to lead to hepatotoxicity [1359-1362] and liver cancer [1363, 1364], and lowering or chelating it is protective [1365, 1366]. Hepcidin may be involved here [1367].

\section{Chronic obstructive pulmonary disorder (COPD) and related lung diseases}

Chronic obstructive pulmonary disease (COPD) is a progressive and chronic disease which is characterised by 
an apparently inexorable decline in respiratory function, exercise capacity, and health status. It is also characterised by periods in which the symptoms are considerably exacerbated [1368-1371]. Such an acute exacerbation of COPD (AECOPD) is defined [1372] as "a sustained worsening of the patient's condition from the stable state and beyond normal day to day variations, that is acute in onset and necessitates a change in regular medication in a patient with underlying COPD". Smoking is a major source of free radicals (and indeed metals [1373]), and is a major cause of COPD. Consequently, there is considerable evidence for the evidence for the involvement of inflammation and ROSs in both the 'stable' and 'exacerbated' stages [1374-1381].

Needless to say, there is also considerable evidence for the significance of exposure to iron [1382] (as well as exposure to other toxic metals [1383]) in the development of COPD and other lung diseases [1384]. Haem oxygenase also appears to be a significant culprit [280], and lung (lavage) iron is increased [1385] while transferrin levels can be considerably lower [1386].

Other lung diseases in which ROS and iron have been implicated include Adult Respiratory Distress Syndrome [1387-1390] and cystic fibrosis [1391].

\section{Smoking}

Tobacco smoke contains many unpleasant and carcinogenic compounds, and that tobacco smoking is a leading cause of carcinoma of the lung and indeed of other organs has become well known since the pioneering epidemiological studies of Doll, Peto and colleagues (e.g. [1392-1395]). Our purpose here is to point out that many of the particles associated with smoking (and also ingested from other sources) are heavily laden with micro-particulate iron, which, as a major catalyst of hydroxyl radical production, undoubtedly is a substantial contributor as well (see e.g. [1373, 1384, 1396-1400]).

\section{Cancer and oncology}

In addition to the issues of smoking, the development of cancer can certainly contain an inflammatory component (e.g. [1401-1425]), and indeed the long-term use of prophylactic anti-inflammatory aspirin lowers colon cancer incidence by $40 \%$ (age-adjusted relative risk = 0.6) [1426] (though note that this may have other sideeffects [1427]). (The well-known association between infectious agents such as $H$. pylori [1428-1430] and e.g. bowel cancer is probably initiated by chronic inflammation.) Given that cells require iron, restricting its supply can also limit the growth of cells, including tumour cells [1431-1442]. Conversely the iron carrier NGAL is overexpressed in tumours [410, 411, 1443], a process mediated via NF- $\kappa$ B [1444] (and see later). Further, the roles of iron, not least in the mutagenic effects of metalcatalysed Fenton chemistry, are also of significance in promoting oncogenesis [1445-1461]. Iron chelators [1438, 1462] are thus a doubly attractive component of anti-cancer therapeutics. The mutagenic, carcinogenic and disease-causing actions of asbestos and related fibres may also be due in significant measure to the ability of the $\mathrm{Fe}(\mathrm{n})$ that they contain to catalyse hydroxyl radical production [1463-1475], while there seem to be complex relations between the likelihood of apoptosis and the differential concentrations of superoxide and $\mathrm{H}_{2} \mathrm{O}_{2}$ [1476, 1477]. Overall, it is becoming increasingly widely recognised that anti-inflammatory agents have a role to play in the treatment of cancers; we would suggest that iron chelation may be a useful component of such treatments.

\section{Malaria}

Just as do tumour cells, the malarial parasite Plasmodium falciparum requires considerable iron for growth, and there is evidence that lowering the amount of available iron provides a promising route to antimalarials (e.g. [1478-1494], but cf. [1495]). Note in this context that iron-catalysed radical formation is also significantly involved in the antimalarial (i.e. cytotoxic) mode of action of artemisinin [1494, 1496-1504], and this reaction is in fact inhibited by iron chelators [1505] such that a combined artemisinin-chelator therapy would be contraindicated.

\section{Antimicrobials}

Lower down the evolutionary scale, and as presaged earlier in the section on bacterial siderophores, microbes require iron for growth, its presence may be limiting even at the scale of global $\mathrm{CO}_{2}$ fixation [1506-1509], its excess can in some circumstances [1510] correlate with infectivity or virulence (see above and e.g. [180, 193, 1493, 1511-1531]), and its chelation in a form not available to bacteria offers a route to at least a bacteriostatic kind of antibiotic or to novel therapies based on the lowering of iron available to microbes by using hepcidin [1532] or NGAL [1533]. Iron chelators are also inhibitory to trypanosomes [1534, 1535], and changes in iron metabolism are also associated with viral infections [1536].

\section{Sepsis leading to organ failure and death: severe inflammatory response syndrome}

It is well known that one consequence of bacterial infection (sepsis) can be septic shock, that this can be mimicked by the Gram-negative bacterial outer membrane component LPS (lipopolysaccharide), and that in 
the worst cases this leads via multiple organ failure to death. However, whatever LPS does it is quite independent of the present of viable (i.e. growing or culturable see $[1537,1538])$ bacteria as the same phenomena leading to multiple organ failure (MOF) are seen without infection [1539, 1540]. Consequently, the recognition of a series of symptoms contingent on this initial inflammatory response has led to the development of the idea of a Systemic Inflammatory Response Syndrome (SIRS) [1541-1551] that leads to the MOF, both via apoptosis [1552] and necrosis [1553, 1554]. There is by now little doubt that these phenomena too are associated with the hyperproduction of ROSs [1546, 1551, 1555-1570]. Circulating free iron is raised in sepsis and related conditions [1400, 1571-1573]. Direct assays of oxidant induced cell death indicate that most 'free' iron is concentrated in lysosomes [1574-1577], that its decompartmentation is substantially involved [1570], and that its chelation can thus prevent cell death [1578-1581].

Many circulating inflammatory factors have been identified as important in the development of septic shock, including cytokines such as Tissue Necrosis Factor (TNF) [1582], and cellular responses via the Toll-Like Receptor are clearly involved in this process [1583, 1584]. However, we would argue that since antibodies against TNF do not inhibit the sequelae of septic shock such as multiple organ failure, the truly damaging agents are caused elsewhere and are likely to involve the iron-mediated production of damaging hydroxyl radicals (see also [1563]).

In this regard, it is especially interesting that the antioxidant melatonin is particularly effective in preventing septic shock [1585-1587], and a variety of suitable antioxidants have shown potential here [1573, 1588-1591], notably in combination with iron chelators [1592, 1593] (and see also [1594, 1595]). As with quite a number of the indications given above, a further link with Fe metabolism is seen in the protective effects of erythropoietin [1596, 1597].

\section{Pro- and anti-oxidants and their contributions to cellular physiology}

A very great many cellular metabolites are redox active within the range of redox potentials realistically accessible to biology (including some molecules such as proline [1598] that are not commonly considered to be redox-active), and it is not our purpose here to list them extensively. Not only their redox potential and status but even their absolute amounts can have profound effects on metabolism [1599]). Our chief point here is that it is the intersection of iron metabolism and oxygen reduction that needs to be the focus, with the 'iron'-catalysed production of hydroxyl radical being the nexus, with the standard redox potential of a redox couple per se being less significant in absolute terms, and the redox potential that a particular redox couple 'feels' being dependent in a complex manner on a variety of thermodynamic and kinetic factors [1229]. Thus, although ascorbate is 'reducing' and an 'antioxidant', its reaction with $\mathrm{O}_{2}$, especially when catalysed by $\mathrm{Fe}(\mathrm{II})$, produces superoxide and thence $\mathrm{OH}^{\bullet}$ radicals that may be pro-oxidant. It is this kind of stepwise multi-electron-transfer phenomenon that explains the otherwise possibly puzzling observation of the oxidant-induced reduction of respiratory chain components (see e.g. [1600, 1601]). Consequently, it is extremely unwise to make pronouncements on the role of 'ROSs' without being quite explicit about which ones are meant.

Thus anything - even an antioxidant - that e.g. by reaction with $\mathrm{O}_{2}$ produces superoxide, peroxide and hydroxyl radicals will turn out to be a pro-oxidant if the flux to superoxide and in particular to hydroxyl radicals is stimulated. Thermodynamically, the 1-electron reduction by ascorbate of dioxygen is disfavoured, with the 2-electron reduction to peroxide being the thermodynamically preferred route. However, such reactions are heavily restricted kinetically in the absence of any catalysts [130]. It is an unfortunate fact that the oxygen-mediated "autoxidation" of ascorbate does in fact occur at considerable rates when it is accelerated by the presence of iron or other transition metal ions [130, 1602-1606]. In a similar vein, 'free' or inadequately liganded $\mathrm{Fe}(\mathrm{II})$ catalyses the production of hydroxyl radicals from oxygen plus a variety of natural biomolecules, including adrenaline (epinephrine) [1607], haemin [1608], and even peptides such as the amyloid- $\beta$ involved in the development of Alzheimer's disease [1009, 1027]. Dietary antioxidants (see below) can therefore act in complex and synergistic ways depending on iron status [1609]. In this regard, the idea of using elemental iron plus ascorbate in food supplements [1610] does not seem a good one.

It should be noted that there are also occasions, e.g. in the decomposition of refractory polymers such as lignin, where such radical production is involved beneficially [1611].

Finally, a variety of molecules can trap hydroxyl radicals, including hippurate [1612], melatonin [1233, 1585, $1586,1613-1631]$ and salicylate [1632].

\section{Antioxidants as therapeutic agents? Should we be including iron chelators in such clinical trials?}

Given the wide recognition of the importance of ROSs in a variety of diseases as described above, many investigators have considered the use of known antioxidants such as vitamins $\mathrm{C}$ (ascorbate) and $\mathrm{E}$ ( $\alpha$-tocopherol) in 
preventative therapy. Although there have been some successes (e.g. [1633]), the results have generally been decidedly mixed, with little clinical benefit (or even actual disbenefit) following from their administration [692, 1588, 1634-1639], e.g. for ALS [1640], atherosclerosis [106, 764], cardiovascular disease [1641-1645], neuroprotection [1646], macular degeneration [1647], pre-eclampsia [1648-1652], critical care medicine [1653], aging [1165, 1168, 1654, 1655], lung disease [1656], elective surgery involving ischaemia-reperfusion [1657], all-cause mortality [1637, 1658], etc. One interpretation for these disappointing results, that is consistent with the general theme of this review, involves the recognition that a variety of antioxidants can in fact act as pro-oxidants and thus actually promote the production of $\mathrm{OH}^{\bullet}$ radicals in the presence of inappropriately or inadequately liganded $\mathrm{Fe}(\mathrm{II})[130,528,1602$, $1604,1659,1660$ ). (One might also comment that the intracellular location of the antioxidants may be an issue, and that the view that targeting them to mitochondria may well have considerable merit [1661-1663].) Thus any predictions about the utility or otherwise of antioxidants need to take into account the amount of 'free' iron present. In particular, we would suggest that future trials of this type might beneficially include appropriate iron chelators, whether alone or with antioxidants.

\section{Liganding and reactivity of $\mathrm{Fe}(\mathrm{n})$}

Given the damage that iron-mediated $\mathrm{OH}^{\bullet}$ radical can create, the question arises as to whether appropriate chelators can inhibit this by inhibiting the production of $\mathrm{OH}^{\circ}$, and while the answer is 'yes' the interpretation of the relevant experiments is not always as clear cut as one would wish [43]. This is because the $\mathrm{OH}^{\bullet}$ radical is so reactive that its production is normally assessed by addition of the putative chelator and observation of its effect on the rate of reaction of a target molecule such as salicylate with the $\mathrm{OH}^{\bullet}$ generated. The ability of a chelator to inhibit such a reaction can then occur not only via a reduction in the rate of $\mathrm{OH}^{\bullet}$ production but by trapping the $\mathrm{OH}^{\bullet}$ itself, as well as by other mechanisms [1664]. This said, there is little doubt that iron chelators can be highly protective, and it is many ways very surprising that their use is not more widespread.

We begin by noting that the reactivity of iron does vary greatly depending upon its liganding environment [71]. Cheng et al. state [72] "Oxygen ligands prefer $\mathrm{Fe}(\mathrm{III})$; thus, the reduction potential of the iron is decreased. Conversely, nitrogen and sulfur ligands stabilize $\mathrm{Fe}(\mathrm{II})$; thus, the reduction potential of the iron is increased. Therefore, chelators with oxygen ligands, such as citrate, promote the oxidation of $\mathrm{Fe}(\mathrm{II})$ to $\mathrm{Fe}(\mathrm{III})$, while chelators that contain nitrogen ligands, such as phenanthroline, inhibit the oxidation of $\mathrm{Fe}(\mathrm{II})$. Many chelators, such as EDTA and Desferal (DFO), will bind both $\mathrm{Fe}(\mathrm{II})$ and Fe- (III); however, the stability constants are much greater for the $\mathrm{Fe}(\mathrm{III})$ chelator complexes. Therefore, these chelators will bind $\mathrm{Fe}$ (II) and subsequently promote the oxidation of the $\mathrm{Fe}(\mathrm{II})$ to $\mathrm{Fe}(\mathrm{III})$ with the concomitant reduction of molecular oxygen to partially reduced oxygen species. Since the maximal coordination number of iron is six, the hexadentate chelators can provide more consistently inert complexes due to their ability to completely saturate the coordination sphere of the iron atom and, consequently, deactivate the "free iron" completely. For example, DFO is a very effective antioxidant in clinical application because of its potential to markedly decrease the redox activity of iron [137]." However, it is not easy to make hexadentate ligands orally active [1665].

Iron typically can coordinate 6 ligands in an octahedral arrangement. Preferential chelation of the $\mathrm{Fe}(\mathrm{II})$ or the Fe (III) form necessarily changes its redox potential as a result of Le Chatelier's principle, and from Marcus theory [1666-1669] the rate of outer-sphere electron transfer reactions is typically related to differences in the free energy change, i.e. the differences in redox potentials of the interacting partners. In addition, it widely recognised that [137] "The tight binding of low molecular \{weight chelators via coordinating ligands such as $\mathrm{O}, \mathrm{N}, \mathrm{S}$ to iron blocks the iron's ability to catalyze redox reactions. Since the maximal coordination number of iron is six, it is often argued that the hexadentate chelators can provide more consistently inert complexes due to their ability to completely saturate the coordination sphere of the $\mathrm{Fe}$ atom. Consequently, a chelator molecule that binds to all six sites of the Fe ion completely deactivates the "free iron". Such chelators are termed "hexidentate" $\{$ sic $\}$, of which desferrioxamine is an example. There are many $\mathrm{Fe}$ chelators that inhibit the reactions of $\mathrm{Fe}$, oxygen, and their metabolites. For example, desferrioxamine ... (DFO) markedly decreases the redox activity of $\mathrm{Fe}$ (III) and is a very effective antioxidant through its ability to bind Fe."

By contrast, bidentate or tridentate chelators that bind to only 2 or 3 of the available iron chelation sites, especially when they bind to both $\mathrm{Fe}(\mathrm{II})$ and $\mathrm{Fe}(\mathrm{III})$, can in fact catalyse redox cycling and thereby promote free radical generation [1437, 1665, 1670, 1671]. Thus, the most potent iron chelators will normally be hexadentate (but may consequently strip iron from iron-containing enzyme and thereby have deleterious side effects). Bi- or tri-dentate ligands should therefore be at saturating concentrations for maximum effect.

Generally, the harder ligands that favour $\mathrm{Fe}(\mathrm{III})$ involve O whereas softer ligands that bind $\mathrm{Fe}(\mathrm{II})$ involve $\mathrm{N}$ and $\mathrm{S}$. The type of ligand also influences the absorption spectrum of 
the ferric form of the chelator, such that conclusions can be drawn about the types of group involved in the complex. These charge transfer bands that appear on ligand binding are at around $340 \mathrm{~nm}$ for carboxylates, around $425 \mathrm{~nm}$ for trishydroxamates, $470 \mathrm{~nm}$ for bis-hydroxamates, $515 \mathrm{~nm}$ for monohydroxamates, around $480 \mathrm{~nm}$ for tris-catecholates, $560 \mathrm{~nm}$ for bis-catecholates and $680 \mathrm{~nm}$ for monocatecholates [173]. In addition, for tris-bidentate complexes the complex can, on an octahedral arrangement, have two different configurations, a left-handed propeller, termed the $\Lambda$-configuration, and a righthanded propeller, the $\Delta$-configuration [173].

\section{Iron chelators - those approved and used clinically}

A number of reviews (e.g. [1437, 1438, 1672-1678]) cover aspects of iron chelators that have had or may have utility clinically.

Whitnall and Richardson [1062] list a number of useful features of an experimentally (and clinically) useful iron chelator. Thus, "A compound suitable for the treatment of neurodegenerative disease should possess a number of qualities, namely (1) strong affinity for FeIII, (2) low molecular weight, (3) lipophilicity high enough to accommodate permeation of cell membranes and the BBB, (4) oral activity, and (5) minimal toxicity [1062]. Also, partly because there are few trivalent ions other than $\mathrm{Fe}(\mathrm{III})$ that the body actually needs, the major synthetic focus has been on the design of FeIII-selective chelators which feature "hard" oxygen donor atoms. Additionally, under aerobic conditions, high-affinity FeIII chelators will tend to chelate FeII to facilitate autoxidation, such that high-affinity FeIII-selective compounds will beneficially bind both FeIII and FeII under most physiological conditions" [1062]. (Note that liophilicity per se may not be relevant, as drugs require carriers to cross membranes [18], and promiscuity and off-target effects increase with lipophilicity [1679].)

Desferrioxamines are nonpeptide hydroxamate siderophores composed of alternating dicarboxylic acid and diamine units. linked by amide bonds. They are produced by many Streptomyces species [1680]. Desferrioxamine $\mathrm{B}$ is a linear (acyclic) substance produced (industrially) by the actinobacterium Streptomyces pilosus [1681], and is widely used as an iron chelator for the prevention and treatment of the effects of iron overload. It is commercially available as desferal (desferrioxamine methane sulphonate), also known as deferoxamine in the USA. It has been very effective in the treatment of a number of diseases, leading to the view that such molecules should have considerable therapeutic potential. A significant disadvantage of DFO is that it does not seem to cross the intestine intact (despite the rather catholic substrate specificity of intestinal peptide transporters [1682-1685]) and must therefore be given intravenously or subcutaneously. By contrast, another chelator known as Deferriprone or L1 does appear to cross cell membranes, but it is only bidentate.

Those with approval for clinical use are few in number and we deal with them first. Table 1 compares them with the 'ideal' properties of a clinically useful iron chelator, while Fig 7 gives the structure of the three most

Table I: Comparison of the main available iron chelators to an ideal chelation drug (modified from [2469])

\begin{tabular}{|c|c|c|c|c|}
\hline & "Ideal chelator" & Deferoxamine & Deferiprone & Deferasirox \\
\hline Route of administration & Oral & $\begin{array}{l}\text { Parenteral, usually } \\
\text { subcutaneous or } \\
\text { intravenous }\end{array}$ & Oral & Oral \\
\hline Plasma half-life & $\begin{array}{l}\text { Long enough to give } \\
\text { constant protection from } \\
\text { labile plasma iron }\end{array}$ & $\begin{array}{l}\text { Short (minutes); requires } \\
\text { constant delivery }\end{array}$ & $\begin{array}{l}\text { Moderate ( }<2 \text { hours). } \\
\text { Requires at least } 3 \text {-times- } \\
\text { per-day dosing }\end{array}$ & $\begin{array}{l}\text { Long, } 8-16 \text { hours; remains } \\
\text { in plasma at } 24 \mathrm{~h}\end{array}$ \\
\hline Therapeutic index & High & $\begin{array}{l}\text { High at moderate doses in } \\
\text { iron-overloaded subjects }\end{array}$ & $\begin{array}{l}\text { Idiosyncratic side effects are } \\
\text { most important }\end{array}$ & $\begin{array}{l}\text { Probably high in iron } \\
\text { overloaded subjects* }\end{array}$ \\
\hline $\begin{array}{l}\text { Molar iron chelating } \\
\text { efficiency; charge of iron (III) } \\
\text { complex }\end{array}$ & High, uncharged & High (hexadentate); charged & Low (bidentate); uncharged & $\begin{array}{l}\text { Moderate (tridentate); } \\
\text { uncharged }\end{array}$ \\
\hline Important side effects & $\begin{array}{l}\text { None or only in } \\
\text { iron-depleted subjects }\end{array}$ & $\begin{array}{l}\text { Auditory and retinal } \\
\text { toxicity; effects on bones } \\
\text { and growth; potential lung } \\
\text { toxicity, all at high doses; } \\
\text { local skin reactions at } \\
\text { infusion sites }\end{array}$ & $\begin{array}{l}\text { Rare but severe } \\
\text { agranulocytosis; mild } \\
\text { neutropenia; common } \\
\text { abdominal discomfort; } \\
\text { erosive arthritis }\end{array}$ & $\begin{array}{l}\text { Abdominal discomfort; } \\
\text { rash or mild diarrhoea } \\
\text { upon initiation of therapy; } \\
\text { mild increased creatinine } \\
\text { level }\end{array}$ \\
\hline $\begin{array}{l}\text { Ability to chelate } \\
\text { intracellular cardiac and } \\
\text { other tissue iron in humans }\end{array}$ & High & $\begin{array}{l}\text { Probably lower than } \\
\text { deferiprone and deferasirox } \\
\text { (it is not clear why) }\end{array}$ & $\begin{array}{l}\text { High in clinical and in in vitro } \\
\text { studies }\end{array}$ & $\begin{array}{l}\text { Insufficient clinical data } \\
\text { available; promising in } \\
\text { laboratory studies }\end{array}$ \\
\hline
\end{tabular}

*Nephrotoxicity that has been observed in non-iron-loaded animals has been minimal in iron-overloaded humans, but effectiveness is demonstrated only at higher end of tested doses, as discussed in [1693]. 


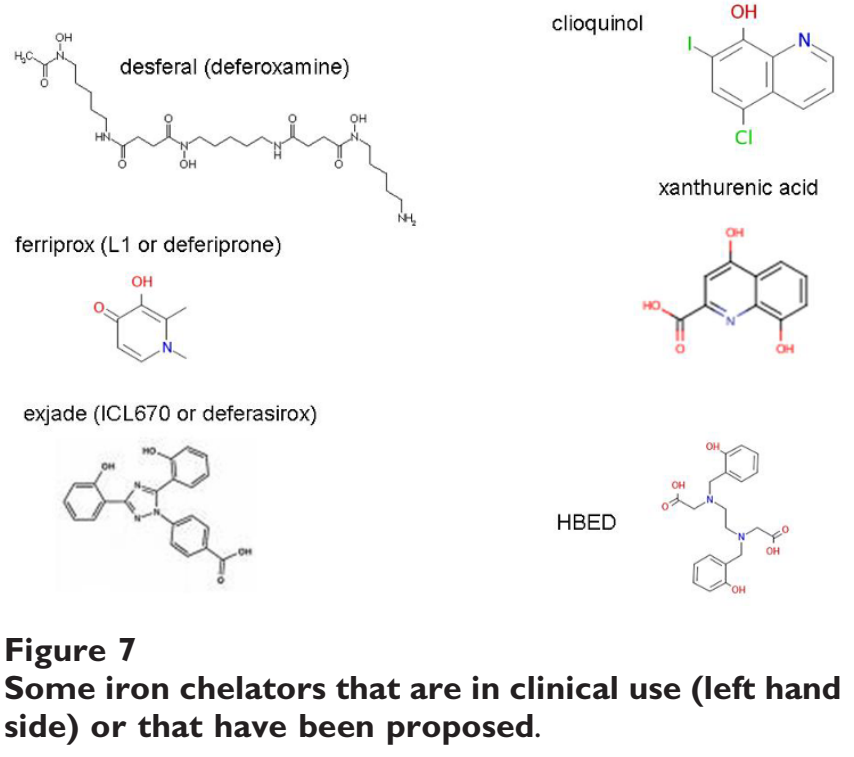

common, viz. desferal (deferoxamine), ferriprox (L1 or deferiprone) and exjade (ICL670 or deferasirox). (Dexrazoxane, a hexadentate chelator, is also marketed [1686].)

Desferal (deferoxamine) is the most used chelator for historical reasons. It is hexadentate but is not orally bioavailable. Ferriprox (deferiprone) is a bidentate ligand (1,2-dimethyl, 3-hydroxypyridin-4-one). It is orally bioavailable although comparatively high doses are required, and it postdates desferal. "Deferiprone has high affinity for iron and interacts with almost all the iron pools at the molecular, cellular, tissue and organ levels. Doses of 50-120 mg/kg/day appear to be effective in bringing patients to negative iron balance" [1687]. It can have somewhat better properties than desferal [1688]. Finally, Exjade (ICL670) (deferasirox) [1689-1702] is the most recent chelator approved for clinical use, and is tridentate. It is orally active, and there is a large bibliography at http://www.exjade.com/utils/ bibliography.jsp?disclaimer=Y. The recommended initial daily dose of Exjade is $20 \mathrm{mg} / \mathrm{kg}$ body weight.

It is clear from Table 1 that in the time evolution from deferoxamine through deferiprone to deferasirox there has been a noticeable improvement in the general properties of iron chelators, although there are few published data on the quantitative structure-activity relationships of candidate molecules that might allow one to design future ones rationally. What is certainly clear is that there is a trade-off in properties, and that appropriate chelators will keep iron levels intermediate, i.e. not too low and not too high (a 'Goldilocks' strategy, if you will), and that hexadentate molecules may correspondingly be too tightly binding and strip iron from important molecules that need it. What is particularly important, as well as a good plasma halflife, is the ability to cross cell membranes, as this is necessary both for oral administration and for ensuring that the chelator in question actually accesses the intracellular 'free' iron pools of interest. Which carriers are used for this in humans in vivo is presently uncertain $[18,1703]$.

Drugs that have been approved for clinical use for other purposes, but that also happen to be iron chelators

The high investment of time, money and intellectual activity necessary to get a drug approved clinically has led to a number of strategies to exploit those that already have been approved and are thus considered 'safe'. One such strategy is the combination therapy of approved drugs that can yet serve for novel indications (e.g. [1704-1708]). Another strategy is to look for antioxidant or iron-binding chemical motifs in drugs that have already been approved for other purposes [1709] (or to measure such properties directly).

Clioquinol (CQ) [1062, 1674, 1710, 1711] (Fig 7) is one existing (anti-parasitic) drug that has been proposed for use as an iron chelator, as it contains the known ironchelating 8-hydroxyquinoline moiety. It has indeed enjoyed some success in this role. However, clioquinol toxicity has been reported if it is used over an extended period [1712] and this may be due to the formation of a Zn-clioquinol chelate [1713].

A particular attraction of such existing drugs is that they are likely to have favourable pharmacokinetics and pharmacodynamics, and in particular are likely to be cell-permeable. Note that despite a widespread assumption that lipophilicity or $\log \mathrm{P}$ is sufficient to account for drug distribution this is not in fact the case, as there are hundreds of natural transporters that drugs can use (e.g. $[18,1714])$. For instance, the iron-chelating 8-hydroxy quinoline motif contained in molecules such as clioquinol is also present in the tryptophan catabolite xanthurenic acid (Fig 7), and it is likely that transmembrane transport of the synthetic drug molecule occurs via natural carriers whose 'normal' role is to transport endogenous but structurally related molecules [18, 1703].

\section{Iron chelators that have been studied but not yet approved}

Given the importance of the field, many academic investigators have sought to develop their own iron chelators that might exhibit the desirable properties listed above. One class of molecule includes 
isonicotinylhydrazones. Thus, pyridoxal isonicotinyl hydrazone (PIH) [1672, 1715-1720] is a promising molecule (also proposed in anti-cancer therapy), although it is hydrolysed both in vivo and in vitro [1721]. Other analogues include salicylaldehyde (SIH) [1722] and 2-hydroxy-1-napthylaldehyde (NIH) isonicotinyl hydrazones. PIH was disclosed before being patented, and is thus seen as having no pharmaceutical (company) interest. Various other derivatives are therefore being considered [1035, 1462], including pyrazinylketone isonicotinoyl hydrazones [1723].

A variety of 8-hydroxyquinolines (8HQs) [1724] have been considered, although as with other bidentate and tridentate ligands that cannot necessarily effect complete liganding of iron there is always a danger that inadequate concentrations might be pro-oxidant (e.g. $[1725,1726])$. One molecule, VK-28, combines various pertinent moieties and has shown some promise in the treatment of neurological disorders [1727-1730]. This strategy of combining drug elements that can hit multiple targets ('polypharmacology' [807, 1731-1734]) has much to commend it, including on theoretical grounds, and we discuss these in the section on systems biology below. Another 8HQ that has elicited interest is O-trensox [1431, 1735-1742].

Other ligands or motifs that might be considered include di-2-pyridylketone-4,4,-dimethyl-3-thiosemicarbazone (Dp44mT), that has been shown to be effective against tumours [1743], 2,2'-dipyridyl, 1,10-phenanthroline [1744, 1745], 2-benzoylpyridine thiosemicarbazones [1746] and thiohydrazones [1747]. HBED (Fig 7) (N, N'-bis-(2-hydroxybenzyl)ethylenediamine- $\mathrm{N}, \mathrm{N}^{\prime}$-diacetic acid) forms a $1: 1$ complex with $\mathrm{Fe}(\mathrm{III})$ but is probably only tetradentate [1748]. It seems not to be very orally active [1749] but may be more effective than is DFO [1750-1752]. Poly-hydroxylated 1,4-naphthoquinones occur as sea urchin pigments and have shown protective effects [1753].

Continuing the theme of polypharmacology, R- $(\alpha)$ lipoic acid [1754-1757] is also an antioxidant, that may in addition act by stimulating other anti-oxidant pathways [1758]. Finally, one interesting area is that of prochelators (e.g. [1759]) in which the oxidant itself triggers the formation of a chelator able to inhibit the Fenton reaction.

\section{Utility of iron chelators in disease amelioration} Therapeutic uses of iron chelators have been widely and usefully reviewed (e.g. [1437, 1489, 1676, 1760-1767]). Many problems remain, such as bioavailability, misdosing [1768] (too little iron as well as too much of it can be bad), toxicity, selectivity and so on, and their design is consequently highly non-trivial [1665, 1670]. Nevertheless, iron chelators have demonstrated therapeutic benefits in Alzheimer's [1674, 1710, 1769-1771], Parkinson's [1037, 1729, 1772], cold-induced brain injury [1773, 1774], coronary disease [714, 797], renal diseases [1775], various kinds of infection [1763] and of course in iron overload diseases [1762, 1767].

As mentioned above, one interesting strategy is to devise chelators that are only activated by oxidative stress $[1760,1776-1779]$. Another is to seek to combine different kinds of functionality in the same molecule. To this end, Youdim and colleagues in particular have developed a series of multifunctional 8-hydroxyquinoline [1740] derivatives that are effective bidentate iron chelators and that seem to show considerable promise in the treatment of a variety of neurodegenerative diseases [1037, 1727-1729, 1780-1784] (see also US Patent 20060234927). In this case the antioxidative mechanism is clearly via chelation since such (8-hydroxyquinoline) molecules are poor scavengers of radicals directly [1785], a fact that also makes them useful scientific tools. As bidentate ligands they cross both cell membranes and the BBB fairly easily (though lipophilicity per se seems not to be important for the biological activity of 8 hydroxyquinoline chelators [1737, 1739]). Importantly, the comparatively weak bidentate binders seem not to have major long-term effects if used carefully [1483, $1762,1786,1787]$

\section{Interaction of xenobiotics with iron metabolism}

As Cherny and colleagues point out [1710], there are many US Pharmacopaeia-registered drugs that, while not being termed chelators, do in fact have both chelating properties and favourable toxicity profiles. Thus we need to recognise potentially both positive and negative interactions between drugs in general and iron metabolism. Any drug that can bind iron can also catalyse the formation of free radicals. Thus, gentamicin can form a gentamicin-iron complex that can lead to toxic symptoms such as hearing loss; this is reversed by iron chelators [1788, 1789]. Existing drugs other than iron chelators may also have effects on iron metabolism [1790], and iron can catalyse their oxidation [1791]. It is not, of course, news that drugs have multiple effects. In this context, we reiterate that some statins, for instance, have chelating properties [893].

Other toxicants might also mediate their damaging effects through iron-catalysed radical formation [1792, 1793]. This in addition to the well-known iron-catalysed, radical-mediated mechanism of toxicity of the viologens such as diquat and paraquat [1794-1799] (whose 
herbicidal activity is in fact inhibited by iron chelators [1800]) and of adriamycin [1801, 1802]. As mentioned above, the carcinogenic action of asbestos may also be due to the ability of the $\mathrm{Fe}(\mathrm{n})$ that it contains to catalyse hydroxyl radical production [1469, 1475], while carcinogenic mycotoxins such as aflatoxin may interact synergistically with iron [1803].

\section{Dietary sources of iron chelators}

There is also a considerable and positive role for nutrients in terms of their chelation of iron. Indeed, polyphenolic compounds, many of which have known health benefits [1804-1813], are widely used as food antioxidants [1814, 1815]. There is of course considerable epidemiological evidence for the benefits of consuming fruit and vegetables that are likely to contain such antioxidants (e.g. [1816-1819]), and - although possibly a minimum this has been popularised as the 'five a day' message (e.g. http://www.fruitsandveggiesmatter.gov/ and http:// www.5aday.nhs.uk/). Even though elements of the 'Mediterranean' diet that are considered to be beneficial are usually assumed to be so on the basis of their antioxidant capabilities (but cf. [1820]), many of the polyphenolic compounds (e.g. flavones, isoflavones, stilbenes, flavanones, catechins (flavan-3-ols), chalcones, tannins and anthocyanidins) [1821-1828] so implicated may also act to chelate iron as well [1073, 1829-1843]. This is reasonable given that many of these polyphenols and flavonoid compounds [1821, 1844-1853] have groups such as the catechol moiety that are part of the known iron-binding elements of microbial siderophores. Examples include flavones such as quercetin [914, 1813, 1829, 1854-1864], rutin [1829, 1857, 1858, 1865, 1866], baicalin [1860, 1867], curcumin [1813, 1868-1872], kolaviron [1873], flavonol [1874], floranol [1875], xanthones such as mangiferin [1876-1879], morin [1876], catechins [1073, 1807, 1838, 1854, 1880, 1881] and theaflavins [1882], as well as procyanidins [1835, $1883]$ and melatonin [1628, 1884-1887]. However, the celebrated (trans-)-resveratrol molecule [1888-1902] may act mainly via other pathways.

A considerable number of studies with non-purified dietary constituents containing the above polyphenolic components have also shown promise in inhibiting diseases in which oxidative stress is implicated [1825, 1903-1906]. For instance in stroke and related neuronal aging and stress conditions, preventative activity can be found in blueberries [1907-1913] (and see [1914]), Ginkgo biloba extract (EGb 761) [1910, 1915, 1916], grapes [1917], green tea [1807, 1918-1921], Mangifera indica extract [1879], strawberries [1907], spinach [1907] and Crataegus [922], while combinations of some these components ('protandim') have been claimed to reduce
ROS levels by stimulating the production of catalase and SOD [1922]. As with pharmaceutical drugs [18, 1923-1925], there are significant problems with bioavailability [1926, 1927], although the necessary measurements are starting to come forward [1804, 1809, 1926-1932]. There is now increasing evidence for the mechanisms with which these dietary components and related natural products and derivatives (often with antiinflammatory, anti-mutagenic or anti-carcinogenic properties) interact with well recognised cellular signalling pathways (e.g. [1402, 1935-1944, 1895, 1945-1952, 1896, 1410, 1953-1960, 1413, 1961-1981, 1913, 1982, 1900, 1933, 1983-1990]).

\section{Role of iron-generated ROSs in cellular signalling and oxidative stress}

Thus, although this is not the focus of the present more physiologically based review, we recognise that many of relationships between ROSs and oxidative stress and overt progressive diseases may be mediated via the inflammatory signalling pathways involved in 'innate immunity' [900, 1991-1993]. NF- $\kappa$ B is an important transcription factor, and the NF- $\kappa$ B system is intimately involved in this signalling [588, 672, 719, 1408, 1409, 1454, 1994-2019].

In the NF- $\kappa$ B system (e.g. [2020-2024]) (Fig 8), NF- $\kappa$ B is normally held inactive in the cytoplasm by binding to an inhibitor $\mathrm{I} \kappa \mathrm{B}$ (often $\mathrm{I} \kappa \mathrm{B} \alpha$ ). Pro-inflammatory cytokines such as TNF- $\alpha$, LPS [2025-2030] and IL-1 [2031] act by binding to receptors at the cell surface and initiating signalling pathways that lead to the activation of a particular kinase, I $\kappa$ B kinase or IKK. This kinase phosphorylates the $\mathrm{I} \kappa \mathrm{B}$ causing it to be released (and ubiquitinated and degraded by the proteasome), allowing the NF- $\kappa \mathrm{B}$ to be translocated to the nucleus where it can activate as many as 300 genes. Simple models of the NF- $\kappa$ B system show the main control elements [2032, 2033] and their synergistic interaction [2034]. The NF- $\kappa \mathrm{B}$ system is implicated in apoptosis [2035, 2036], aging [1199], and in diseases such as cancer $[1405,1444,1454,1808,2037-2040]$, arthritis [2040-2043] and a variety of other diseases [2044]. Antioxidants such as vitamin E $[552,2017]$ and melatonin [2045-2049] are at least partially protective. Oxidative stress seems to act upstream of IKK [2014], on I $\kappa \mathrm{B} \alpha$ directly [2050] and in the p38 MAP kinase pathway [1993, 2014, 2051], and there is also evidence that at least some of the statins act on the PI3K-akt and NF- $\kappa \mathrm{B}$ pathways too [819, 883, 2052-2060]. A considerable number of inhibitors of the NF- $\kappa$ B system exist [2055], many exhibiting cross-reactivity [1734].

The induction of NF- $\kappa \mathrm{B}$ by ROSs appears to involve a coupling via the glutathione system [2007, 2035, 2036, 2061-2078] (and see also [2079, 2080]). 


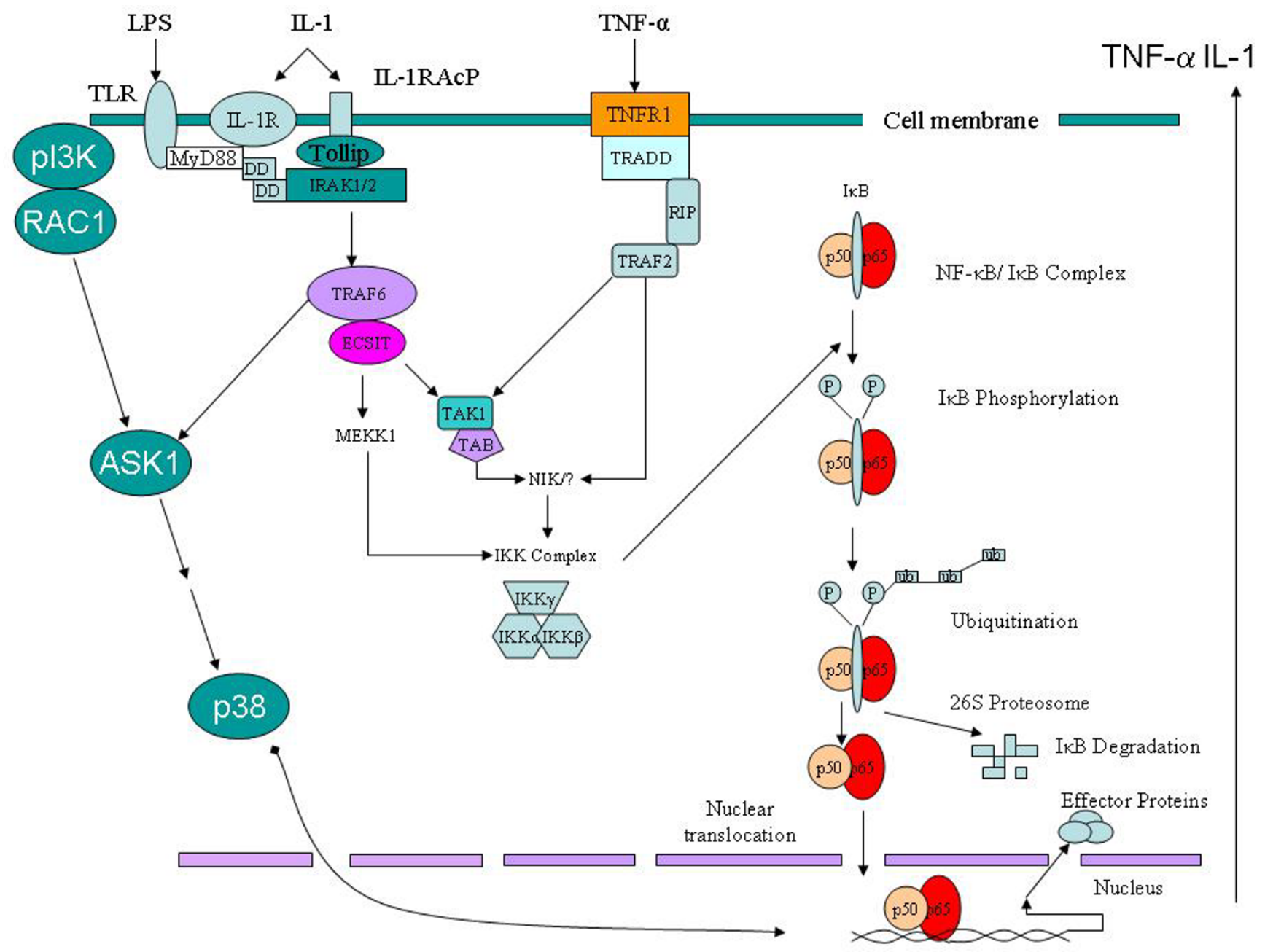

\section{Figure 8}

An overview of cellular signaling using the NF- $\kappa$ B and p38 systems. Note that some of the extracellular effectors that mediate NF- $\kappa$ B activation are themselves produced and secreted as a result of the activation, potentially creating an autocatalytic system.

A variety of studies have shown that iron is involved in these signalling processes [1839, 1996, 2015, 2081-2085], probably again acting upstream of the IKK [557, 2083, 2086, 2087].

Interestingly, there is interplay between the NF- $\kappa \mathrm{B}$ pathway and the regulation of NGAL [404, 405, 2088, 2089], ferritin [2030] and hepcidin [2090], presumably acting as a negative feedback as the cell tries to control and ligand its free $\mathrm{Fe}(\mathrm{n})$ in the face of oxidative stress caused by the release of free iron [2091].

\section{The systems biology of ROS generation and activity}

It is not news that most major changes in physiological states have multigenic or multifactorial origins (e.g.
[2092, 2093]). This means, as an inevitable consequence, that we need to recognize that their observation requires a systems approach, and that most diseases are therefore in fact to be seen as systems or network diseases [631, 2094-2102]. Changes in individual reaction steps (or even single manipulations) can change the levels of scores or hundreds of transcripts [2103], proteins [2104] or metabolites [725]. In this regard, small molecule (metabolomics) measurements have especial advantages for capturing network organisation, including on theoretical grounds [2105-2112].

If we consider just one variable of present relevance, the quantity of hydroxyl radical, the amount that is able to react with proteins, lipids and DNA is clearly determined by a huge number of reactions, whether directly or 
otherwise - not only the concentrations of reagents and enzyme that directly catalyse its formation and destruction but by everything else that affects their concentration and activity, and so on. This is of course well established in biology in the formalism of metabolic control analysis (MCA) (see e.g. [2113-2120]), and was recognized over 30 years ago in Morris' prescient review on anaerobes [2121]. Modern systems theories of aging (e.g. [1175, 1180, 2120, 2122] and above) (Fig 9) also recognize physiological progression as being determined in terms of a balance between 'good' and 'bad' factors. MCA and related formalisms can be seen as theories of sensitivity analysis, which in many cases can be normalized such that an overall output function can be described quantitatively in terms of the relative contributions of each of its component steps (e. g. [2123-2127]). In MCA the normalized local sensitivities are known as control coefficients, and the sum of the concentration-control coefficients $=0$, in other words in the steady state the rate of production and consumption of a particular entity is in balance and all reactions can contribute to it to some degree. The concentration-control coefficient describes this degree quantitatively. It is now possible to produce appropriate quantitative representations of metabolic networks using quite sparse kinds of information (in fact just the stoichiometry and network structure [2128]), and thereby provide initial estimates for more sophisticated fitting algorithms (e.g. [2129-2132]. Indeed, the analysis of the properties and behaviour of networks is at the core of modern systems biology (e.g. [2095, 2133-2140]).

A corollary of such considerations is that to decrease the amount of damage caused by $\mathrm{OH}^{\bullet}$ (or any other) radicals we need both to decrease their production and

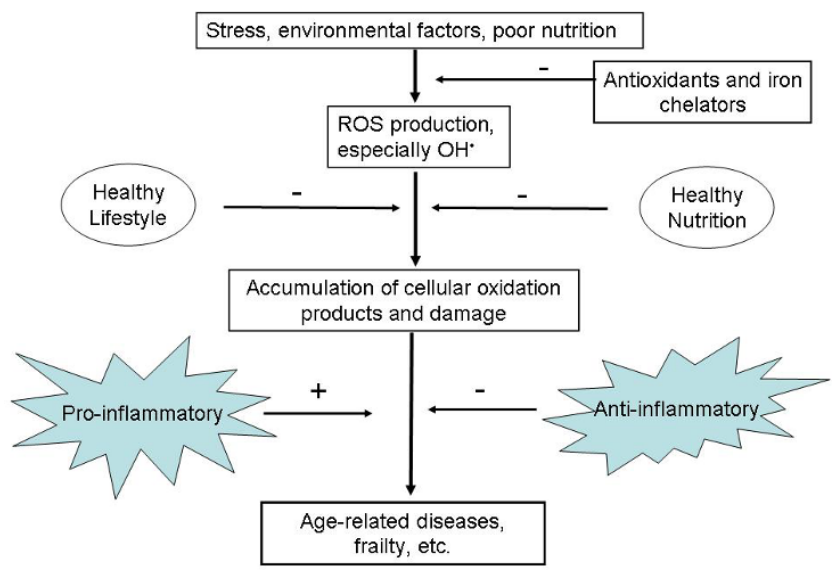

Figure 9

General view of the role of iron, antioxidants and ROSs in aging and degenerative processes. Some of the decay may be ameliorated by lifestyle and dietary means. Based in part on [II75]. increase their removal to harmless substances [2141], and that on general grounds [1706-1708, 2142] such a strategy (for instance of combining a cell-permeable iron chelator with a cell-permeable antioxidant) might be expected to give a synergistic response. Even determining the means of cell permeability and tissue distribution turns out to be a systems biology problem in which we need to know the nature and activity of all the carriers that are involved [18, 1703, 1714, 2143]. At all events, it is undoubtedly the case that the steady-state rate of production of a molecule such as the hydroxyl radical is controlled or affected by a considerable number of steps. These minimally include the multiple reactions of the mitochondrial respiratory chain and the various oxidases producing superoxide and peroxide, the activities of catalase and SOD enzymes that together can remove them, protective reactions such as heat-shock proteins, and most pertinently to the present review a large number of reactions involved in the metabolism and safe liganding of iron that help determine the rate at which $\mathrm{OH}^{\bullet}$ is produced.

It is also pertinent to enquire as to why we are now seeing so many of these progressive diseases, and as to what may be their causes. Undoubtedly the simple fact of improved longevity is one [165] as damage accumulates. However, we note that anything that decreases the amount of unliganded iron, such as decreasing the total dietary iron intake e.g. from red meat, must be helpful [1156, 2144].

\section{Anti-inflammatory cytokines; the example of erythropoietin}

We have above adduced considerable evidence that decreasing the amount of hydroxyl radical by any means is valuable, whether by removing initially generated ROSs such as superoxide and peroxide or by chelating poorly liganded iron in a way that stops these ROSs forming the hydroxyl radical. While pro-inflammatory cytokines can themselves increase ROS production and modulate the activities of signaling pathways such as NF$\kappa \mathrm{B}$ and p38, there are also anti-inflammatory cytokines. A particularly interesting example is that of erythropoietin (also discussed above as being protective in a number of iron-mediated diseases).

Erythropoietin was originally recognized via its role in erythropoiesis [2145-2147] (hence its name, of course), but it has become evident that it has many other roles, and in particular it is observed phenomenologically that erythropoietin (and non-erthyropoetic derivatives) is protective in a number of inflammatory conditions that accompany many diseases such as those listed above [940, 942, 2148-2155]. These included 
cardiovascular disease [721, 722, 2156-2175], stroke and other related neurological diseases [924-926, 928, 929, 933, 937, 938, 2155, 2176-2204], diabetic neuropathy [2205], kidney injury [2173, 2206-2212], intestinal injury [2213] and shock (both septic and non-septic) [1596, 1597, 2214].

The question then arises as to how it is doing this mechanistically, and the proximate answer is that it (and other anti-inflammatory agents, e.g. [1808, 2215, 2216]) seem to act via many of the same signalling pathways as do inflammatory agents [943, 2150, 2217-2226]. There is evidence that it can help maintain superoxide dismutase activity [2214, 2227], invoke haem oxygenase [2228], and in particular - from the perspective of this review - that it may remove poorly liganded iron [2229] and interact with hydroxyl radical directly [2230-2233].

It is notable that appropriate levels of erythropoietin appear not only to be efficacious but to be safe, even in pregnancy [2234-2241]. Erythropoietin may itself be a marker of hypoxia and oxidative stress in pregnancy [531, 2242-2245], consistent with a view that the body is attempting to deal with these problems by creating antiinflammatory cytokines.

\section{Hypoxia-inducible factor (HIF)}

Although I am mainly not concentrating on genetic regulatory aspects in this review, the HIF [2246, 2247] does deserve some mention, since many of the syndromes described above are accompanied by hypoxia, and this causes levels of the HIF to increase. HIF is a transcription factor that can activate a considerable number of genes, including VEGF [1951, 2246-2250]. In contrast to the constitutive expression of HIF- $1 \alpha$, HIF$1 \beta$ protein levels are regulated in response to the cellular oxygen concentration [2251]. The active HIF is the HIF$1 \alpha \beta$ heterodimer [2252]. HIF couples anoxia to innate immunity via the NF- $\kappa \mathrm{B}$ system [2253]. In particular, HIF effects (via hepcidin) the mobilisation or iron and can cause the expression of inflammatory cytokines such as IL-1, IL-6 and TNF- $\alpha$ [2254-2256] under conditions (hypoxia) where superoxide and peroxide production are likely to be increased, and consequently increases sepsis (in that HIF-knockout mice are resistant to LPSinduced sepsis [2254, 2255]). By contrast, induction of HIF (and the genes that it activates) can effect neuroprotection [2252, 2257]. HIF also appears to have a significant role in placental development, and defective HIF expression may be involved in preeclampsia and intra-uterine growth retardation [435, 2246, 2258]. Qutub and colleagues provide useful models [2259, 2260] of HIF activation under a variety of conditions of iron, $\mathrm{O}_{2}, 2$-oxoglutarate and other factors.

\section{Autocatalysis, positive feedback and Systems Biology}

What has emerged in recent years is a recognition that the structure (i.e. topology) of the modules of metabolic and signalling networks, somewhat independent of the individual activities of their components, can have a profound controlling influence on their behaviour (e.g. [2107, 2134, $2135,2261,2262]$ ). Classically, negative feedback structures are considered to confer stability, while positive feedbacks tend to have the opposite effect. However, negative feedbacks with delay loops can cause oscillations [2022, 2109] while some kinds of positive feedback loops can confer stability $[2262,2263]$. However, there is no doubt that structures in which a damaging agent causes the production of a second damaging agent that itself catalyses the production of the first or a separate damaging agent can exhibit a runaway kind of damage. This is exactly what can happen with iron and superoxide since $\mathrm{Fe}(\mathrm{n})$ can be liberated from ferritin by superoxide radicals and then catalyse the production of further hydroxyl radical by increasing the amount of free iron (Fig 6). A similar effect can occur with $\mathrm{Fe}-\mathrm{S}$ proteins in SOD-deficiency [1148], with the degradation of mitochondria by radical damage leading to further production of radicals [28, 30, 2264], and the effects of oxidative stress on iron storage [2265]. This again illustrates the importance of acting at multiple points in a network to control these kinds of damage. Exactly the same is true of the IL- 1 and TNF- $\alpha$ systems in which IL- 1 or TNF- $\alpha$ (oxidative stress) acting on one cell can effect the secretion of further IL-1/TNF- $\alpha$ that can act on adjacent cells (Fig 8), of the hypoxia-dependent increase in both ROSs and serum iron mediated by hepcidin (Fig 3), the autocatalytic synergy between overfeeding, inflammation and (pre-)diabetes, and of the peroxide/iron pair that are liberated when frataxin is deficient (see above). It is these kinds of synergistic effects and autocatalytic cycles that are the hallmark of the major and progressive effects on human physiology that are seen in these kinds of system. Indeed, one might comment that such multi-site and autocatalytic effects are required to overwhelm normal defences precisely because human metabolic and signalling systems are 'robust' systems that have evolved topologies that are resistant or robust to parameter changes (see e.g. [2266-2291]).

\section{Predictive biology}

It is often considered (e.g. [2292, 2293]) that a desirable feature of a scientific idea is its ability to make useful predictions, and while this is not in fact a particularly well founded philosophical principle, it probably is of value to set out a couple of 'predictions' that follow from the present analysis. One prominent feature of the above is the primacy of the iron-catalysed production of the damaging hydroxyl radical, and thus a test of the involvement of these kinds of reactions in the various physiological and pathological states to which we allude 
is the prediction that they should be accompanied by markers of oxidative stress characteristic of reactions of endogenous metabolites with the hydroxyl radical. While it is not that easy to disentangle the complex reactions of ROSs with biomolecules [43, 1055], at least the following appear to be a result of reactions involving $\mathrm{OH}^{\bullet}[2294,2295]:$ 8-oxo-2'-deoxyguanosine (oxo ${ }^{8} \mathrm{dG}$ ) [98, 2296, 2297], 8-oxo-7,8- dihydro-2'-deoxyguanosine $[90,92]$ and thymine glycol $[92,2294]$.

Another set of predictions from the systems biology perspective [1704-1708, 1731, 2138, 2291, 2298-2310] is that combinations of chemical agents (or manipulations such as those of transcription factors that affect multiple steps in a pathway [1906] or modulation of multiple gene products [2311], or both [2312, 2313]) will be far more efficacious, for instance in modulating iron-catalysed oxidative stress and its sequelae, than will be the use of 'magic bullet' single agents. Such combinations of 'causes' do not have to be guessed $a$ priori but can be obtained via inferencing techniques ( $e$. g. [2314-2320]) - for a recent example see [2321]. The nonlinear behaviour of biochemical networks also serves to explain the bell-shaped dose-response curves underpinning the hormesis [2322-2326] often observed.

Iron-mediated oxidative stress is arguably the cause of much of the inflammation typically observed in biological systems, often further mediated via pro-inflammatory cytokines. Another major prediction that comes from the above then is that molecules that are anti-inflammatory, whether widely recognised as such or not, should have beneficial effects in syndromes for which they have not necessarily been tested. An obvious set of candidates in this regard are to be found among the statins, since it is now clear that they have important anti-inflammatory properties (see above). Thus, there are already indications that as well as their established benefits in cardiovascular disease (e.g. [804, 2327]) they may exert benefit in a huge variety of syndromes [838], including sepsis [839, 2060, 2328-2340], heart failure [2341], pain perception [2342], lupus and related diseases [1293, 2343], diabetes [877, 2344], rheumatoid arthritis [866, 869, 890, 2345-2350], kidney disease [2351-2353], inflammatory skin disease [2354], emphysema [2355], ischaemia-reperfusion injury [2356], stroke [864, 872, 2357-2364], traumatic brain injury [2365-2367], neurodegenerative diseases [860-862, 920, 1294, 2059, 2368-2384], neurotoxicity [2385] and cancer [2386-2400].

\section{Concluding remarks and quo vadis}

"Actually, the orgy of fact extraction in which everybody is currently engaged has, like most consumer economies, accumulated a vast debt. This is a debt of theory and some of us are soon going to have an exciting time paying it back - with interest, I hope." [2401].

"But one thing is certain: to understand the whole you must look at the whole" [2402]

"If you are not thinking about your experiments on a whole-genome level you are going to be a dinosaur". J. Stamatoyannopoulos, quoted in [2403].

While it is less common for scientists to publish 'negative' results ('there was no effect of some agent on some process'), and there has been a tendency to seek to falsify specific hypotheses rather than to paint a big picture [2404], there is no doubt that the sheer weight of positive evidence can be persuasive in leading one to a view. As Bertrand Russell put it [2405], "When one admits that nothing is certain one must, I think, also admit that some things are much more nearly certain than others." However, as mentioned above, the huge volume of scientific activity has in many ways led to a 'balkanisation' of the literature [2406] in which scientists deal with the problem of the deluge of published papers by necessarily ignoring most of them. This is no longer realistic (nor necessary) in an age of post-genomics, the internet, Web 2.0 and systems biology, and when we are starting to move to integrative (if distributed) models of organisms (including humans) at a whole organ, genome or whole organism scale $[118,2135$, 2407-2416]. The 'digital human' is thus an important research goal [2410, 2415-2417]. Expression profiling atlases are becoming increasingly widespread (e.g. [2418-2423]), and one can anticipate using these straightforwardly to extend these 'generalised' (sub) cellular network models in a tissue-specific manner [2424]. With the ability to exchange models of biochemical networks in a principled way [2425-2428], when they are marked up appropriately (e.g. $[3,4])$, we can expect to begin to reason about them automatically $[118,2429]$, such that we may soon look forward to an era in which we can recognise the commonalities across a variety of different subfields - a specific message of the present overview. Thus, while iron and metabolism should be considered in the context of other processes that may be contributing to the disorders discussed, and it is evident that they are intimately involved in many disease processes, therapies derived for one of the inflammatory diseases listed above may well have benefit in some of the others where their underlying 'causes' are the same. The 'mass collaboration' agenda (e.g. [2430-2435]), in which dispersed agents contribute their different skills to the solution of a complex problem, may well help this happen effectively. Developments in distributed workflow technology, such as the Taverna [22-26, 2436, 2437]http://taverna.sourceforge.net/ and the 
myExperiment [2438]http://www.myexperiment.org/ environments, represent an intellectually important approach. Important too to this endeavour will be Open Access initiatives $[1,2439,2440]$ and institutional and other repositories [2441,2442] of full-text papers. This will help to build an accurate picture of the biochemical networks operating in both normal and diseased states (and see [2443]), preferably marked up semantically as in [118], and hence, by modelling them [2263], where best to consider interventions. In order to develop and exploit this distributed approach, it will also be necessary for those generating them to make their data and metadata available (preferably in a semantically marked up way), probably as Web Services (e.g. [24, 2444-2452]), and to give greater scientific weight to those involved in bio-curation [119] as they will be an increasingly important part of the scientific landscape. Modern sequencing instruments, for instance (e.g. [2453-2457]) are generating quality data at truly enormous rates [2458], and innovative but computationally demanding algorithms are required to deal with them (e.g. [2459]). In particular, however, we need tools for manipulating and visualising biochemical models [2110, 2410, 2429, 2460]. As well as storing these models (e.g. as SBML or CellML [2461]) in a file format, it is also convenient to store them in a database format, such as the B-net database developed by Mendes and colleagues $[118,2462]$. Federated annotation protocols such as the Distributed Annotaton Scheme (see e.g. [2463]) allow data from heterogeneous sources to be combined, while other integrative/distributed architectures such as ONDEX [2464] and Utopia [2465-2468] are similarly showing considerable promise for integrative systems biology.

Given the widely dispersed communities that have been referenced herein, and the future requirement for integrating knowledge generated throughput the world, a programme for understanding the combinatorial roles of poorly liganded iron and reactive oxygen species in the aetiology of many diseases, as set out in the above, appears to be prototypical for the kinds of new approaches to doing science that we may anticipate in the eras of Web 2.0 and the Semantic Web.

\section{Abbreviations}

AECOPD: Acute Exacerbation of Chronic Obstructive Pulmonary Disorder; AKI: Acute Kidney Injury; ALS: Amyotrophic Lateral Sclerosis; AMD: Age-related Macular Degeneration; BBB: Blood-brain barrier; ChEBI: Chemical Entities of Biological Interest; CQ: Clioquinol; COPD: Chronic Obstructive Pulmonary Disorder; DFO: Desferrioxamine; Dp44mT: di-2-pyridylketone4,4,-dimethyl-3-thiosemicarbazone; EDTA:
Ethylenediamine-tetraacetic Acid; FA: Friedrich's ataxia; FPN1: Ferroportin-1; HBED: N,N'-bis-(2-hydroxybenzyl) ethylenediamine-N, $\mathrm{N}^{\prime}$-diacetic acid; HCP1: Heme Carrier Protein-1; HIF: Hypoxia-Inducible Factor; HO1: Heme Oxygenase-1; HMG-CoA: Hydroxymethyl glutaryl Coenzyme A; Hp: Hephaestin; 8HQs: A variety of 8-hydroxyquinolines; IBD: Inflammatory Bowel Disease; InChI: International Chemical Identifier; IKK: I $\kappa$ B kinase; KEGG: Kyoto Encyclopedia of Genes and Genomes; L1: Deferriprone; LFA-1: Leukocyte Function Antigen-1; LIP: Labile Iron Pool; LIPID: Long-Term Intervention with Pravastatin in Ischaemic Disease; LPS: Lipopolysaccharide; MOF: Multiple organ failure; NF- $\kappa \mathrm{B}$ : Nuclear Factor $\kappa \mathrm{B}$; NGAL: Neutrophil Gelatinase-Associated Lipocalin (Also known as lipocalin-2 or siderocalin); NIH: 2-hydroxy-1-napthylaldehyde isonicotinyl hydrazone; PE: Pre-eclampsia; PIH: Pyridoxal isonicotinyl hydrazone; PPAR: Peroxisome Proliferator Activated Receptor; RA: Rheumatoid Arthritis; ROS: Reactive Oxygen Species; SBML: Systems Biology Markup Language; SIH: Salicylaldehyde isonicotinyl hydrazone; SIRS: Systemic Inflammatory Response Syndrome; SLE: Systemic Lupus Erythematosus; SMILES: Simplified Molecular Input Line Entry Specification; SOD: Superoxide Dismutase; Tf: Transferrin; TNF: Tissue Necrosis Factor; VEGF: Vascular Endothelial Growth Factor.

\section{Competing interests}

The author declares that he has no competing interests.

\section{Acknowledgements}

I thank Jon Barasch (Columbia University) for drawing my attention to the role of NGAL in human physiology, and Phil Baker, David Brough and Louise Kenny for many further useful and enjoyable discussions. I thank Katya Tarasova for considerable assistance with literature gathering and Julie Cowley for assistance with the proofs. Much of my work when this was written has been supported by the UK BBSRC and EPSRC, for which I am most grateful, and I am also a recipient of funding from the British Heart Foundation. The funders had no role in study design, data collection and analysis, decision to publish, or preparation of the manuscript. This is a contribution from the Manchester Centre for Integrative Systems Biology http://www.mcisb.org.

\section{References}

I. Harnad S, Brody T, Vallieres F, Carr L, Hitchcock S, Gingras Y, Oppenheim C, Hajjem C and Hilf ER: The access/impact problem and the green and gold roads to open access: An update. Serials Review 2008, 34:36-40.

2. Hull D, Pettifer SR and Kell DB: Defrosting the digital library: bibliographic tools for the next generation web. PLoS Comput Biol 2008, 4:el000204, doi:I0.I37|/journal.pcbi.1000204.

3. Ananiadou $\mathrm{S}$ and McNaught $\mathrm{J}$ : Text mining in biology and biomedicine.Artech House, London; 2006.

4. Ananiadou S, Kell DB and Tsujii Ji: Text Mining and its potential applications in Systems Biology. Trends Biotechnol 2006, 24:57|-579.

5. Jensen LJ, Saric J and Bork P: Literature mining for the biologist: from information retrieval to biological discovery. Nat Rev Genet 2006, 7:119-29.

6. Smalheiser NR: The Arrowsmith project: 2005 status report. LNCS 2005, 3735:26-43. 
7. Hristovski D, Peterlin B, Mitchell JA and Humphrey SM: Using literature-based discovery to identify disease candidate genes. Int J Med Inform 2005, 74:289-98.

8. Kostoff RN: Systematic acceleration of radical discovery and innovation in science and technology. Technol Forecasting Soc Change 2006, 73:923-936.

9. Yetisgen-Yildiz $M$ and Pratt W: Using statistical and knowledgebased approaches for literature-based discovery. J Biomed Informatics 2006, 39:600-6I I.

10. Kostoff RN: Validating discovery in literature-based discovery. I Biomed Inform 2007, 40:448-50, author reply 450-2.

II. Kostoff RN, Briggs MB, Solka JL and Rushenberg RL: Literaturerelated discovery (LRD): Methodology. Technol Forecast Soc Change 2008.

12. Cronin B: Bibliometrics and beyond: Some thoughts on webbased citation analysis. Journal of Information Science 200 I, 27: I-7.

13. Kasztler $\mathrm{A}$ and Leitner $\mathrm{KH}$ : Bibliometric analysis and visualisation of intellectual capital. J Universal Comp Sci 2002, 8:5I 6-525.

14. Tabah AN: Literature dynamics: Studies on growth, diffusion, and epidemics. Annu Rev Inf Sci Technol 1999, 34:249-286.

15. Börner K, Chen CM and Boyack KW: Visualizing knowledge domains. Annual Review of Information Science and Technology 2003, 37: 179-255

16. Errami M, Hicks JM, Fisher W, Trusty D, Wren JD, Long TC and Garner HR: Déjà vu: a study of duplicate citations in Medline. Bioinformatics 2008, 24:243-9.

17. Kell DB: Iron behaving badly: inappropriate iron chelation as a major contributor to the aetiology of vascular and other progressive inflammatory and degenerative diseases.2008 http://arxiv.org/ftp/arxiv/papers/0808/0808.1371.pdf.

18. Dobson PD and Kell DB: Carrier-mediated cellular uptake of pharmaceutical drugs: an exception or the rule? Nat Rev Drug Discov 2008, 7:205-220.

19. Smalheiser NR, Torvik VI, Bischoff-Grethe A, Burhans LB, Gabriel M, Homayouni R, Kashef A, Martone ME, Perkins GA, Price DL, Talk AC and West R: Collaborative development of the Arrowsmith two node search interface designed for laboratory investigators. J Biomed Discov Collab 2006, I:8.

20. Smalheiser NR and Swanson DR: Using ARROWSMITH: a computer-assisted approach to formulating and assessing scientific hypotheses. Comput Methods Programs Biomed 1998, 57:149-53.

21. Doms A and Schroeder M: GoPubMed: exploring PubMed with the Gene Ontology. Nucleic Acids Res 2005, 33:W783-6.

22. Oinn T, Addis M, Ferris J, Marvin D, Senger M, Greenwood M Carver T, Glover K, Pocock MR, Wipat A and Li P: Taverna: a tool for the composition and enactment of bioinformatics workflows. Bioinformatics 2004, 20:3045-54.

23. Oinn T, Li P, Kell DB, Goble C, Goderis A, Greenwood M, Hull D, Stevens R, Turi D and Zhao J: Taverna/myGrid: aligning a workflow system with the life sciences community. Workflows for e-Science: scientific workflows for Grids Springer, Guildford: Taylor IJ, Deelman E, Gannon DB, Shields M 2007, 300-319.

24. Hull D, Wolstencroft K, Stevens R, Goble C, Pocock MR, Li P and Oinn T: Taverna: a tool for building and running workflows of services. Nucleic Acids Res 2006, 34:W729-32.

25. Oinn T, Greenwood M, Addis M, Alpdemir MN, Ferris J, Glover K, Goble C, Goderis A, Hull D, Marvin D, Li P, Lord P, Pocock MR, Senger $M$, Stevens R, Wipat $A$ and Wroe $C$ : Taverna: lessons in creating a workflow environment for the life sciences. Concurrency and Computation: Practice \& Experience 2006, I 8: 1067-I I00.

26. Goble C, Wolstencroft K, Goderis A, Hull D, Zhao J, Alper P, Lord P, Wroe C, Belhajiame K, Turi D, Stevens R, Oinn T and De Roure D: Knowledge discovery for biology with Taverna: producing and consuming semantics in the Web of Science. Semantic Web: revolutionising knowledge discovery in the life sciences Springer, New York: Baker CJO, Cheung K-H 2007.

27. Chance $B$, Sies $\mathrm{H}$ and Boveris A: Hydroperoxide metabolism in mammalian organs. Physiol Rev 1979, 59:527-605.

28. Lenaz G: Role of mitochondria in oxidative stress and ageing. Biochim Biophys Acta 1998, I366:53-67.

29. Barja G: Mitochondrial oxygen radical generation and leak: sites of production in states 4 and 3, organ specificity, and relation to aging and longevity. J Bioenerg Biomembr I999, 3 I:347-66.

30. Kowaltowski AJ and Vercesi AE: Mitochondrial damage induced by conditions of oxidative stress. Free Radic Biol Med 1999, 26:463-7I.
31. Cadenas $\mathrm{E}$ and Davies $\mathrm{KJ}$ : Mitochondrial free radical generation, oxidative stress, and aging. Free Radic Biol Med 2000, 29:222-30

32. Lenaz G, D'Aurelio M, Merlo Pich M, Genova ML, Ventura B, Bovina C, Formiggini G and Parenti Castelli G: Mitochondria bioenergetics in aging. Biochim Biophys Acta 2000, I 459:397-404.

33. Raha $\mathrm{S}$ and Robinson $\mathrm{BH}$ : Mitochondria, oxygen free radicals, disease and ageing. Trends Biochem Sci 2000, 25:502-8.

34. Lenaz G: The mitochondrial production of reactive oxygen species: mechanisms and implications in human pathology. IUBMB Life 200I, 52:159-64.

35. Raha $\mathrm{S}$ and Robinson $\mathrm{BH}$ : Mitochondria, oxygen free radicals, and apoptosis. Am J Med Genet 200I, 106:62-70.

36. Dröge W: Free radicals in the physiological control of cell function. Physiol Rev 2002, 82:47-95.

37. Taylor ER, Hurrell F, Shannon RJ, Lin TK, Hirst J and Murphy MP: Reversible glutathionylation of complex I increases mitochondrial superoxide formation. I Biol Chem 2003 278: $19603-10$.

38. Turrens JF: Mitochondrial formation of reactive oxygen species. J Physiol 2003, 552:335-44.

39. Adam-Vizi $\mathrm{V}$ : Production of reactive oxygen species in brain mitochondria: Contribution by electron transport chain and non-electron transport chain sources. Antioxidants \& Redox Signaling 2005, 7: I |40-I| 49.

40. Andreyev AY, Kushnareva YE and Starkov AA: Mitochondrial metabolism of reactive oxygen species. Biochemistry (Mosc) 2005, 70:200-14

4I. Balaban RS, Nemoto S and Finkel T: Mitochondria, oxidants, and aging. Cell 2005, I 20:483-495.

42. Adam-Vizi $V$ and Chinopoulos $C$ : Bioenergetics and the formation of mitochondrial reactive oxygen species. Trends Pharmacol Sci 2006, 27:639-45.

43. Halliwell B and Gutteridge JMC: Free Radicals in Biology and Medicine Oxford University Press, Oxford; 42006.

44. Orrenius S, Gogvadze A and Zhivotovsky B: Mitochondrial oxidative stress: Implications for cell death. Annual Review of Pharmacology and Toxicology 2007, 47:143-I83.

45. Fato R, Bergamini C, Leoni S, Strocchi P and Lenaz G: Generation of reactive oxygen species by mitochondrial complex I: implications in neurodegeneration. Neurochem Res 2008 , 33:2487-501

46. Babior BM: Phagocytes and oxidative stress. Am J Med 2000, 109:33-44

47. Cave AC, Brewer AC, Narayanapanicker A, Ray R, Grieve DJ, Walker $S$ and Shah AM: NADPH oxidases in cardiovascular health and disease. Antioxidants \& Redox Signaling 2006, 8:69l-728.

48. Bedard $\mathrm{K}$ and Krause KH: The NOX family of ROS-generating NADPH oxidases: physiology and pathophysiology. Physiol Rev 2007, 87:245-3।3.

49. Brown JM, Terada LS, Grosso MA, Whitmann GJ, Velasco SE, Patt A Harken $\mathrm{AH}$ and Repine JE: Xanthine oxidase produces hydrogen peroxide which contributes to reperfusion injury of ischemic, isolated, perfused rat hearts. J Clin Invest 1988, 8 I:|297-|30|

50. Granger DN: Role of xanthine oxidase and granulocytes in ischemia reperfusion injury. Amer J Physiol I988, 255: $\mathrm{HI} 269-\mathrm{HI} 275$

5I. Linas SL, Whittenburg D and Repine JE: Role of xanthine oxidase in ischemia reperfusion injury. Amer J Physiol 1990, 258: F7II-F7I6.

52. Thompson-Gorman SL and Zweier JL: Evaluation of the role of xanthine oxidase in myocardial reperfusion injury. J Biol Chem 1990, 265:6656-6663.

53. Adkins WK and Taylor AE: Role of xanthine oxidase and neutrophils in ischemia-reperfusion injury in rabbit lung. J Appl Physiol 1990, 69:2012-2018.

54. Müller MJ, Vollmar B, FriedI HP and Menger MD: Xanthine oxidase and superoxide radicals in portal triad crossclampinginduced microvascular reperfusion injury of the liver. Free Radic Biol Med 1996, 21:189-97.

55. Imlay JA: Cellular defenses against superoxide and hydrogen peroxide. Annu Rev Biochem 2008, 77:755-76.

56. Sundaresan M, Yu ZX, Ferrans VJ, Irani K and Finkel T: Requirement for generation of $\mathrm{H}_{2} \mathrm{O}_{2}$ for platelet-derived growth factor signal transduction. Science 1995, 270:296-9.

57. Finkel T: Oxidant signals and oxidative stress. Curr Opin Cell Biol 2003, I 5:247-54.

58. Rhee SG: Cell signaling. H2O2, a necessary evil for cell signaling. Science 2006, 3i 2: I882-3. 
59. Miller EW, Tulyathan O, Isacoff EY and Chang CJ: Molecular imaging of hydrogen peroxide produced for cell signaling. Nat Chem Biol 2007, 3:263-7.

60. Forman HJ, Fukuto JM, Miller T, Zhang H, Rinna A and Levy S: The chemistry of cell signaling by reactive oxygen and nitrogen species and 4-hydroxynonenal. Arch Biochem Biophys 2008, 477: 183-95.

6I. Halliwell B and Gutteridge JM: Oxygen toxicity, oxygen radicals, transition metals and disease. Biochem J 1984, 2 19:|-|4.

62. Halliwell $B$ and Gutteridge JMC: Role of free radicals and catalytic metal ions in human disease - an overview. Meth Enzymol 1990, I86: I-85.

63. Galaris D and Pantopoulos $K$ : Oxidative stress and iron homeostasis: mechanistic and health aspects. Crit Rev Clin Lab Sci 2008, 45: I-23.

64. Buzan T: How to mind map Thorsons, London; 2002.

65. Fridovich I: Superoxide radical and superoxide dismutases. Annu Rev Biochem 1995, 64:97-II2.

66. Wardman P and Candeias LP: Fenton chemistry: An introduction. Rad Res 1996, |45:523-53|.

67. Kehrer JP: The Haber-Weiss reaction and mechanisms of toxicity. Toxicology 2000, |49:43-50.

68. Hershko $C$ and Weatherall DJ: Iron-chelating therapy. Crit Rev Clin Lab Sci 1988, 26:303-45.

69. Minotti G: Sources and role of iron in lipid peroxidation. Chem Res Toxicol 1993, 6:134-146.

70. Rayman MP, Barlis J, Evans RW, Redman CW and King LJ: Abnormal iron parameters in the pregnancy syndrome preeclampsia. Am J Obstet Gynecol 2002, 187:4| 2-8.

7I. Welch KD, Davis TZ, Van Eden ME and Aust SD: Deleterious ironmediated oxidation of biomolecules. Free Radic Biol Med 2002, 32:577-83.

72. Cheng $\mathrm{Z}$ and $\mathrm{Li} \mathrm{Y}$ : What is responsible for the initiating chemistry of iron-mediated lipid peroxidation: an update. Chem Rev 2007, 107:748-66.

73. Tsukahara $\mathrm{H}$ : Biomarkers for oxidative stress: Clinical application in pediatric medicine. Curr Med Chem 2007, |4:339-35|

74. Evans MD, Dizdaroglu M and Cooke MS: Oxidative DNA damage and disease: induction, repair and significance. Mut Res 2004, 567: $|-6|$.

75. Valko M, Izakovic M, Mazur M, Rhodes CJ and Telser J: Role of oxygen radicals in DNA damage and cancer incidence. Mol Cell Biochem 2004, 266:37-56.

76. David SS, O'Shea VL and Kundu S: Base-excision repair of oxidative DNA damage. Nature 2007, 447:94I-50.

77. Stadtman ER and Oliver CN: Metal-catalyzed oxidation of proteins - physiological consequences. J Biol Chem I991, 266:2005-2008.

78. Davies MJ, Fu S, Wang H and Dean RT: Stable markers of oxidant damage to proteins and their application in the study of human disease. Free Radic Biol Med I999, 27:I I5I-63.

79. Davies MJ: The oxidative environment and protein damage. Biochim Biophys Acta 2005, 1703:93-109.

80. Terman A and Brunk UT: Lipofuscin. Int J Biochem Cell Biol 2004, 36: $1400-1404$.

81. Jung $T$, Bader $N$ and Grune $T$ : Lipofuscin: formation, distribution, and metabolic consequences. Ann N Y Acad Sci 2007, I I | 9:97-I I I.

82. Halliwell B: Free radicals and antioxidants: a personal view. Nutr Rev 1994, 52:253-65.

83. De Zwart LL, Meerman JHN, Commandeur JNM and Vermeulen NPE: Biomarkers of free radical damage applications in experimental animals and in humans. Free Rad Biol Med 1999, 26:202-226.

84. Kohen $R$ and Nyska A: Oxidation of biological systems: oxidative stress phenomena, antioxidants, redox reactions, and methods for their quantification. Toxicol Pathol 2002, 30:620-50.

85. Tarpey MM, Wink DA and Grisham MB: Methods for detection of reactive metabolites of oxygen and nitrogen: in vitro and in vivo considerations. Am J Physiol Regul Integr Comp Physiol 2004, 286:R43 I-44.

86. Halliwell $B$ and Whiteman $M$ : Measuring reactive species and oxidative damage in vivo and in cell culture: how should you do it and what do the results mean? Br J Pharmacol 2004, 142:23|-55.

87. Cherubini A, Ruggiero C, Polidori MC and Mecocci P: Potential markers of oxidative stress in stroke. Free Radical Biology and Medicine 2005, 39:84I-852.
88. Shulaev $\mathrm{V}$ and Oliver DJ: Metabolic and proteomic markers for oxidative stress. New tools for reactive oxygen species research. Plant Physiol 2006, I41:367-72.

89. Serkova NJ, Reisdorph NA and van Patot MCT: Metabolic markers of hypoxia: Systems biology application in biomedicine. Toxicology Mechanisms and Methods 2008, I 8:8I-95.

90. Aust AE and Eveleigh JF: Mechanisms of DNA oxidation. Proc Soc Exp Biol Med 1999, 222:246-52.

91. Cooke MS, Lunec $J$ and Evans MD: Progress in the analysis of urinary oxidative DNA damage. Free Radic Biol Med 2002, 33: $1601-1614$.

92. Dizdaroglu $M$, Jaruga $P$, Birincioglu $M$ and Rodriguez $H$ : Free radical-induced damage to DNA: Mechanisms and measurement. Free Radical Biology and Medicine 2002, 32: I I02-III5.

93. Neeley WL and Essigmann JM: Mechanisms of formation, genotoxicity, and mutation of guanine oxidation products. Chem Res Toxicol 2006, 19:491-505.

94. Hwang ES and Bowen PE: DNA damage, a biomarker of carcinogenesis: Its measurement and modulation by diet and environment. Crit Rev Food Sci Nutrition 2007, 47:27-50.

95. Shin CS, Moon BS, Park KS, Kim SY, Park SJ, Chung MH and Lee HK: Serum 8-hydroxy-guanine levels are increased in diabetic patients. Diabetes Care 200I, 24:733-7.

96. Loft S, Fischer-Nielsen A, Jeding IB, Vistisen $K$ and Poulsen HE: 8-Hydroxydeoxyguanosine as a urinary biomarker of oxidative DNA damage. J Toxicol Environ Health 1993, 40:391-404.

97. Migliore L, Fontana I, Colognato R, Coppede F, Siciliano G and Murri L: Searching for the role and the most suitable biomarkers of oxidative stress in Alzheimer's disease and in other neurodegenerative diseases. Neurobiol Aging 2005, 26:587-95.

98. Bolin $\mathrm{C}$ and Cardozo-Pelaez F: Assessing biomarkers of oxidative stress: Analysis of guanosine and oxidized guanosine nucleotide triphosphates by high performance liquid chromatography with electrochemical detection. J Chromatogr B Analyt Technol Biomed Life Sci 2007.

99. Yamada S, Funada T, Shibata N, Kobayashi M, Kawai Y, Tatsuda E, Furuhata $A$ and Uchida K: Protein-bound 4-hydroxy-2-hexenal as a marker of oxidized n-3 polyunsaturated fatty acids. J Lipid Res 2004, 45:626-634.

100. Petersen DR and Doorn JA: Reactions of 4-hydroxynonenal with proteins and cellular targets. Free Radic Biol Med 2004 37:937-45.

101. Montuschi P, Ciabattoni G, Paredi P, Pantelidis P, du Bois RM, Kharitonov SA and Barnes PJ: 8-Isoprostane as a biomarker of oxidative stress in interstitial lung diseases. Amer J Resp Crit Care Med 1998, 158:1524-1527.

102. Montuschi P, Collins JV, Ciabattoni G, Lazzeri N, Corradi M, Kharitonov SA and Barnes PJ: Exhaled 8-isoprostane as an in vivo biomarker of lung oxidative stress in patients with COPD and healthy smokers. Amer J Resp Crit Care Med 2000, 162: I 175-I I77.

103. Schwedhelm E and Boger RH: Application of gas chromatography-mass spectrometry for analysis of isoprostanes: their role in cardiovascular disease. Clin Chem Lab Med 2003, 4|:|552-6I.

104. Davì G, Falco A and Patrono C: Determinants of F2-isoprostane biosynthesis and inhibition in man. Chem Phys Lipids 2004 128: 149-63.

105. Montuschi P, Barnes PJ and Roberts LJ 2nd: Isoprostanes: markers and mediators of oxidative stress. FASEB ] 2004, | 8: |79|-800.

106. Morrow JD: Quantification of isoprostanes as indices of oxidant stress and the risk of atherosclerosis in humans. Arterioscler Thromb Vasc Biol 2005, 25:279-86.

107. Montuschi P, Barnes P and Roberts LJ 2nd: Insights into oxidative stress: the isoprostanes. Curr Med Chem 2007, I4:703-I7.

108. Mori TA, Croft KD, Puddey IB and Beilin LJ: Analysis of native and oxidized low-density lipoprotein oxysterols using gas chromatography mass spectrometry with selective ion monitoring. Redox Report 1996, 2:25-34.

109. Murphy RC and Johnson KM: Cholesterol, reactive oxygen species, and the formation of biologically active mediators. J Biol Chem 2008, 283: I552I-5.

I I0. Janero DR: Malondialdehyde and thiobarbituric acid reactivity as diagnostic indexes of lipid peroxidation and peroxidative tissue injury. Free Radic Biol Med 1990, 9:5 I 5-540.

III. Gieseg SP, Crone EM, Flavall EA and Amit Z: Potential to inhibit growth of atherosclerotic plaque development through modulation of macrophage neopterin/7,8-dihydroneopterin synthesis. Br J Pharmacol 2008, I 53:627-35. 
I 12. Herce-Pagliai C, Kotecha S and Shuker DEG: Analytical methods for 3-nitrotyrosine as a marker of exposure to reactive nitrogen species: A review. Nitric Oxide-Biology and Chemistry 1998, 2:324-336.

113. Bian K, Gao Z, Weisbrodt N and Murad F: The nature of hemel iron-induced protein tyrosine nitration. Proc Natl Acad Sci USA 2003, I00:5712-7.

114. Duncan MW: A review of approaches to the analysis of 3-nitrotyrosine. Amino Acids 2003, 25:35I-36I.

II5. Ryberg $\mathrm{H}$ and Caidahl K: Chromatographic and mass spectrometric methods for quantitative determination of 3-nitrotyrosine in biological samples and their application to human samples. J Chromatogr B 2007, 85 I: |60-I7|

I 16. Cao EH and Wang J): Oxidative Damage to DNA - Levels of Thymine Glycol and Thymidine Glycol in Neoplastic Human Urines. Carcinogenesis 1993, I4:1359-1362.

I I7. Makropoulos W, Kocher K, Heintz B, Schwarz ER, Mertens PR and Stefanidis I: Urinary thymidine glycol as a biomarker for oxidative stress after kidney transplantation. Renal Failure 2000, 22:499-510.

I I8. Herrgård MJ, Swainston N, Dobson P, Dunn WB, Arga KY, Arvas M, Blüthgen N, Borger S, Costenoble R, Heinemann M, Hucka M, Le Novère $N$, Li $P$, Liebermeister $W$, Mo ML, Oliveira AP, Petranovic $D$, Pettifer S, Simeonidis E, Smallbone K, Spasić I, Weichart D, Brent R, Broomhead DS, Westerhoff HV, Kirdar B, Penttilä M, Klipp E, Palsson B $\varnothing$, Sauer U, Oliver SG, Mendes P, Nielsen J and Kell DB: A consensus yeast metabolic network obtained from a community approach to systems biology. Nature Biotechnol 2008, 26: I|55-I|60.

1 19. Howe D, Costanzo M, Fey P, Gojobori T, Hannick L, Hide W, Hill DP, Kania R, Schaeffer M, St Pierre S, Twigger S, White $O$ and Yon Rhee S: Big data: The future of biocuration. Nature 2008, 455:47-50.

120. Brooksbank C, Cameron G and Thornton J: The European Bioinformatics Institute's data resources: towards systems biology. Nucleic Acids Res 2005, 33:D46-53.

121. Kanehisa M, Goto S, Hattori M, Aoki-Kinoshita KF, Itoh M, Kawashima S, Katayama T, Araki $M$ and Hirakawa $M$ : From genomics to chemical genomics: new developments in KEGG. Nucleic Acids Res 2006, 34:D354-7.

122. Weininger D: SMILES, a chemical language and information system .I. Introduction to methodology and encoding rules. J Chem Inf Comput Sci 1988, 28:31-36.

123. Stein SE, Heller SR and Tchekhovski D: An open standard for chemical structure representation - the IUPAC chemical identifier. Proc 2003 Nimes Int Chem Info Conf 2003, I3 I-I43.

124. Coles SJ, Day NE, Murray-Rust P, Rzepa HS and Zhang Y: Enhancement of the chemical semantic web through the use of InChI identifiers. Organic \& Biomolecular Chemistry 2005, 3:1832-1834.

125. Casher O and Rzepa HS: SemanticEye: a semantic web application to rationalize and enhance chemical electronic publishing. J Chem Inf Model 2006, 46:2396-4II

126. Monge A, Arrault A, Marot $C$ and Morin-Allory L: Managing, profiling and analyzing a library of $\mathbf{2 . 6}$ million compounds gathered from 32 chemical providers. Mol Divers 2006, 10:389-403.

127. Knox C, Shrivastava S, Sothard P, Eisner R and Wishart DS: Biospider: a web server for automating metabolome annotations. Pac Symp Biocomputing 2007, I 2: |45-156.

128. Klinger R, Kolářik C, Fluck J, Hofmann-Apitius $M$ and Friedrich CM: Detection of IUPAC and IUPAC-like chemical names. Bioinformatics 2008, 24:i268-76.

129. Chavarria ME, Lara-Gonzalez L, Gonzalez-Gleason A, GarciaPaleta Y, Vital-Reyes VS and Reyes A: Prostacyclin/thromboxane early changes in pregnancies that are complicated by preeclampsia. Am J Obstet Gynecol 2003, I 88:986-92.

130. Miller DM, Buettner GR and Aust SD: Transition metals as catalysts of "autoxidation" reactions. Free Radic Biol Med I990, 8:95-108.

131. Stohs SJ and Bagchi D: Oxidative mechanisms in the toxicity of metal ions. Free Rad Biol Med 1995, 18:321-336.

132. Meneghini R: Iron homeostasis, oxidative stress, and DNA damage. Free Radical Biology and Medicine 1997, 23:783-792.

133. Hubel CA: Dyslipidemia, iron, and oxidative stress in preeclampsia: assessment of maternal and feto-placental interactions. Semin Reprod Endocrinol 1998, 16:75-92.

134. Aisen P, Enns C and Wessling-Resnick M: Chemistry and biology of eukaryotic iron metabolism. Int J Biochem Cell Biol 200I, 33:940-959.
135. Reddy MB and Clark L: Iron, oxidative stress, and disease risk. Nutr Rev 2004, 62:120-4.

136. Thompson KJ, Shoham S and Connor JR: Iron and neurodegenerative disorders. Brain Res Bull 200 I, 55: I55-64.

137. Valko M, Morris $H$ and Cronin MTD: Metals, toxicity and oxidative stress. Curr Med Chem 2005, I 2: I I6I-I 208.

138. Carbonell $T$ and Rama R: Iron, oxidative stress and early neurological deterioration in ischemic stroke. Current Medicinal Chemistry 2007, |4:857-874.

139. Dunn LL, Rahmanto YS and Richardson DR: Iron uptake and metabolism in the new millennium. Trends Cell Biol 2007, 17:93-100.

140. Weinberg ED: Exposing the hidden dangers of iron: what every medical professional should know about the impact of iron on the disease process Cumberland House; 2004

141. Brewer GJ: Iron and copper toxicity in diseases of aging, particularly atherosclerosis and Alzheimer's disease. Exp Biol Med 2007, 232:323-335.

142. Valko M, Leibfritz D, Moncol J, Cronin MTD, Mazur M and Telser J: Free radicals and antioxidants in normal physiological functions and human disease. Int J Biochem Cell Biol 2007, 39:44-84

143. Winterbourn CC: Reconciling the chemistry and biology of reactive oxygen species. Nat Chem Biol 2008, 4:278-86.

144. Qian SY and Buettner GR: Iron and dioxygen chemistry is an important route to initiation of biological free radical oxidations: an electron paramagnetic resonance spin trapping study. Free Radic Biol Med 1999, 26: |447-56.

145. Brown GC and Borutaite V: Nitric oxide, mitochondria, and cell death. IUBMB Life 200I, 52:189-95.

146. Gomes A, Fernandes $E$ and Lima J: Use of fluorescence probes for detection of reactive nitrogen species: A review. J Fluorescence 2006, 16:1 19-139.

147. Yokoyama H, Kuroiwa H, Yano $R$ and Araki T: Targeting reactive oxygen species, reactive nitrogen species and inflammation in MPTP neurotoxicity and Parkinson's disease. Neurol SCi 2008, 29:293-301.

148. Moncada S, Palmer RMJ and Higgs EA: Nitric-Oxide - Physiology, Pathophysiology, and Pharmacology. Pharmacological Reviews 1991, 43:109-142.

149. Beckman JS, Beckman TW, Chen J, Marshall PA and Freeman BA: Apparent hydroxyl radical production by peroxynitrite: implications for endothelial injury from nitric oxide and superoxide. Proc Natl Acad Sci USA 1990, 87: 1620-4.

150. Radi R, Cassina A, Hodara R, Quijano C and Castro L: Peroxynitrite reactions and formation in mitochondria. Free Radic Bio Med 2002, 33:|45|-64.

I5I. Rubbo $H$ and O'Donnell V: Nitric oxide, peroxynitrite and lipoxygenase in atherogenesis: mechanistic insights. Toxicology 2005, 208:305-17.

152. Zimmet JM and Hare JM: Nitroso-redox interactions in the cardiovascular system. Circulation 2006, I | 4: | 53 |-44.

153. Pacher P, Beckman JS and Liaudet L: Nitric oxide and peroxynitrite in health and disease. Physiol Rev 2007, 87:3 I5-424.

154. Pavlovic R and Santaniello E: Peroxynitrite and nitrosoperoxycarbonate, a tightly connected oxidizing-nitrating couple in the reactive nitrogen-oxygen species family: new perspectives for protection from radical-promoted injury by flavonoids. J Pharm Pharmacol 2007, 59: 1687-95.

155. Aslan M, Ryan TM, Adler B, Townes TM, Parks DA, Thompson JA Tousson A, Gladwin MT, Patel RP, Tarpey MM, Batinic-Haberle I, White $C R$ and Freeman BA: Oxygen radical inhibition of nitric oxide-dependent vascular function in sickle cell disease. Proc Natl Acad Sci 2001, 98: I5215-15220.

156. O'Donnell VB and Freeman BA: Interactions between nitric oxide and lipid oxidation pathways - Implications for vascular disease. Circulation Research 200I, 88:|2-2|.

157. Lancaster JR Jr: Protein cysteine thiol nitrosation: maker or marker of reactive nitrogen species-induced nonerythroid cellular signaling? Nitric Oxide 2008, 19:68-72.

158. Vaz SM and Augusto $O$ : Inhibition of myeloperoxidasemediated protein nitration by tempol: Kinetics, mechanism, and implications. Proc Natl Acad Sci USA 2008, I 05:8I 9I-6.

159. Brown GC and Bal-Price A: Inflammatory neurodegeneration mediated by nitric oxide, glutamate, and mitochondria. Mol Neurobiol 2003, 27:325-55

160. Kim YM, Chung HT, Simmons RL and Billiar TR: Cellular nonheme iron content is a determinant of nitric oxide-mediated apoptosis, necrosis, and caspase inhibition. J Biol Chem 2000 , 275: |0954-6| 
16I. Galli SJ, Tsai M and Piliponsky AM: The development of allergic inflammation. Nature 2008, 454:445-54.

162. Finkel T: Radical medicine: treating ageing to cure disease. Nat Rev Mol Cell Biol 2005, 6:97I-6.

163. Finch CE: The biology of human longevity: inflammation, nutrition and aging in the evolution of lifespans Academic Press, Amsterdam; 2007.

164. Serrano $M$ and Blasco MA: Cancer and ageing: convergent and divergent mechanisms. Nat Rev Mol Cell Biol 2007, 8:715-22.

165. Brown GM: The living end: the future of death, aging and immortality Macmillan, London; 2008.

166. Renshaw JC, Robson GD, Trinci APJ, Wiebe MG, Livens FR, Collison D and Taylor RJ: Fungal siderophores: structures, functions and applications. Mycol Res 2002, I 06: I I 23-I | 42.

167. Haas H: Molecular genetics of fungal siderophore biosynthesis and uptake: the role of siderophores in iron uptake and storage. Applied Microbiology and Biotechnology 2003, 62:3 I6-330.

168. Haas H, Eisendle $M$ and Turgeon BG: Siderophores in fungal physiology and virulence. Annu Rev Phytopathol 2008, 46: |49-87.

169. Johnson L: Iron and siderophores in fungal-host interactions. Mycol Res 2008, I I 2: I 70-83

170. Raymond KN, Muller G and Matzanke BF: Complexation of iron by siderophores - a review of their solution and structural chemistry and biological function. Top Curr Chem 1984, 1 23:49-102.

17I. Guerinot ML: Microbial iron transport. Annu Rev Microbiol I994, 48:743-72.

172. Jurado RL: Iron, infections, and anemia of inflammation. Clin Infect Dis 1997, 25:888-95.

173. Drechsel H and Jung G: Peptide siderophores. Journal of Peptide Science 1998, 4:|47-|8|.

174. Ratledge $C$ and Dover LG: Iron metabolism in pathogenic bacteria. Annu Rev Microbiol 2000, 54:88I-94I.

175. Boukhalfa $\mathrm{H}$ and Crumbliss $\mathrm{AL}$ : Chemical aspects of siderophore mediated iron transport. Biometals 2002, I 5:325-339.

176. Braun $V$ and Braun $M$ : Active transport of iron and siderophore antibiotics. Curr Opin Microbiol 2002, 5:194-201.

177. Brown JS and Holden DW: Iron acquisition by Gram-positive bacterial pathogens. Microbes Infect 2002, 4: I|49-56.

178. Cornelis P and Matthijs S: Diversity of siderophore-mediated iron uptake systems in fluorescent pseudomonads: not only pyoverdines. Environ Microbiol 2002, 4:787-798.

179. Crosa JH and Walsh CT: Genetics and assembly line enzymology of siderophore biosynthesis in bacteria. Microbiology and Molecular Biology Reviews 2002, 66:223.

180. Marx J]: Iron and infection: competition between host and microbes for a precious element. Best Pract Res Clin Haematol 2002, I 5:4II-26.

18I. Winkelmann G: Microbial siderophore-mediated transport. Biochem Soc Trans 2002, 30:691-6.

182. Andrews SC, Robinson AK and Rodriguez-Quinones F: Bacterial iron homeostasis. FEMS Microbiol Rev 2003, 27:215-37.

183. Faraldo-Gómez JD and Sansom MSP: Acquisition of siderophores in Gram-negative bacteria. Nat Rev Mol Cell Biol 2003, 4:105-116.

184. Raymond KN, Dertz EA and Kim SS: Enterobactin: an archetype for microbial iron transport. Proc Natl Acad Sci USA 2003, 100:3584-8.

185. Wandersman C and Delepelaire P: Bacterial iron sources: From siderophores to hemophores. Annual Review of Microbiology 2004, 58:61 I-647.

186. Abergel RJ, Wilson MK, Arceneaux JE, Hoette TM, Strong RK, Byers BR and Raymond KN: Anthrax pathogen evades the mammalian immune system through stealth siderophore production. Proc Natl Acad Sci USA 2006, I 03: 18499-503.

187. Fischbach MA, Lin HN, Liu DR and Walsh CT: How pathogenic bacteria evade mammalian sabotage in the battle for iron. Nature Chemical Biology 2006, 2: 132-138.

188. Grass G: Iron transport in Escherichia coli: All has not been said and done. Biometals 2006, 19:159-172.

189. Miethke $M$ and Marahiel MA: Siderophore-based iron acquisition and pathogen control. Microbiol Mol Biol Rev 2007 , 7|:4|3-5|.

190. Genco CA and Dixon DW: Emerging strategies in microbial haem capture. Molecular Microbiology 2001, 39: I-II.

191. Loomis LD and Raymond KN: Solution equilibria of enterobactin and metal enterobactin complexes. Inorganic Chemistry |991, 30:906-91|.

192. Stintzi A, Barnes C, Xu J and Raymond KN: Microbial iron transport via a siderophore shuttle: a membrane ion transport paradigm. Proc Natl Acad Sci USA 2000, 97:10691-6.
193. Braun V: Iron uptake mechanisms and their regulation in pathogenic bacteria. Int J Med Microbiol 200I, 29 I:67-79.

194. Cendrowski S, MacArthur $W$ and Hanna P: Bacillus anthracis requires siderophore biosynthesis for growth in macrophages and mouse virulence. Mol Microbiol 2004, 5 I:407-4I7.

195. Schaible ME and Kaufmann SHE: Iron and microbial infection. Nat Rev Microbiol 2004, 2:946-953.

196. Ong ST, Ho JZS, Ho B and Ding JL: Iron-withholding strategy in innate immunity. Immunobiology 2006, 2 I I:295-3 I4.

197. Challis GL and Ravel J: Coelichelin, a new peptide siderophore encoded by the Streptomyces coelicolor genome: structure prediction from the sequence of its non-ribosomal peptide synthetase. FEMS Microbiol Lett 2000, I87: I I I-4.

198. Fernandez-Pol JA: Isolation and characterization of a siderophore-like growth factor from mutants of SV40-transformed cells adapted to picolinic acid. Cell I978, I4:489-99.

199. Jones RL, Peterson CM, Grady RW and Cerami A: Low molecular weight iron-binding factor from mammalian tissue that potentiates bacterial growth. J Exp Med 1980, I 5 I:4 I8-28.

200. Primosigh JV and Thomas ED: Studies on the partition of iron in bone marrow cells. J Clin Invest 1968, 47:|473-82.

20I. Jacobs A: Low molecular weight intracellular iron transport compounds. Blood 1977, 50:433-9.

202. Hershko C, Graham G, Bates GW and Rachmilewitz EA: Nonspecific serum iron in thalassaemia: an abnormal serum iron fraction of potential toxicity. Br J Haematol 1978, 40:255-63.

203. Graham G, Bates GW, Rachmilewitz EA and Hershko C: Nonspecific serum iron in thalassemia: quantitation and chemical reactivity. Am J Hematol 1979, 6:207-I7.

204. Craven CM, Alexander J, Eldridge M, Kushner JP, Bernstein S and Kaplan J: Tissue distribution and clearance kinetics of nontransferrin-bound iron in the hypotransferrinemic mouse: a rodent model for hemochromatosis. Proc Natl Acad Sci 1987. 84:3457-6I.

205. Fontecave $M$ and Pierre JL: Iron metabolism - the low molecular mass iron pool. Biology of Metals 1991, 4:133-135.

206. Kaplan J: Mechanisms of cellular iron acquisition: another iron in the fire. Cell 2002, I I I:603-6.

207. Ganz T: Hepcidin, a key regulator of iron metabolism and mediator of anemia of inflammation. Blood 2003, 1 02:783-788.

208. Finch C: Regulators of iron balance in humans. Blood 1994 84: $1697-702$.

209. Kohgo Y, Ikuta K, Ohtake T, Torimoto $Y$ and Kato J: Body iron metabolism and pathophysiology of iron overload. Int J Hematol 2008, 88:7-I5.

210. Crichton RR and Ward RJ: Iron metabolism-new perspectives in view. Biochemistry 1992, 3 I: I I 255-64.

211. Andrews NC: Medical progress: Disorders of iron metabolism. New England Journal of Medicine 1999, 341:|986-1995.

212. Beard JL, Dawson $H$ and Piñero DJ: Iron metabolism: a comprehensive review. Nutr Rev 1996, 54:295-317.

213. Lieu PT, Heiskala M, Peterson PA and Yang Y: The roles of iron in health and disease. Mol Aspects Med 200I, 22:I-87.

214. Chung JY and Wessling-Resnick M: Molecular mechanisms and regulation of iron transport. Crit Rev Clin Lab Sci 2003, 40: $151-182$.

215. Frazer DM and Anderson G]: The orchestration of body iron intake: how and where do enterocytes receive their cues? Blood Cells Molecules and Diseases 2003, 30:288-297.

216. Knutson $M$ and Wessling-Resnick $M$ : Iron metabolism in the reticuloendothelial system. Crit Rev Biochem Mol Biol 2003, 38:6I-88.

217. McArdle HJ, Danzeisen R, Fosset $C$ and Gambling L: The role of the placenta in iron transfer from mother to fetus and the relationship between iron status and fetal outcome. Biometals 2003, I 6: $161-7$.

218. Hentze MW, Muckenthaler MU and Andrews NC: Balancing acts: molecular control of mammalian iron metabolism. Cell 2004, I I 7:285-97.

219. Andrews NC: Molecular control of iron metabolism. Best Pract Res Clin Haematol 2005, I 8: 159-69.

220. Papanikolaou G and Pantopoulos K: Iron metabolism and toxicity. Toxicol Appl Pharmacol 2005, 202:199-2II.

22I. Taketani S: Aquisition, mobilization and utilization of cellular iron and heme: Endless findings and growing evidence of tight regulation. Tohoku J Exp Med 2005, 205:297-3।8.

222. Donovan A, Roy $C N$ and Andrews NC: The ins and outs of iron homeostasis. Physiology (Bethesda) 2006, 21:I I5-23. 
223. Ganz $T$ and Nemeth E: Regulation of iron acquisition and iron distribution in mammals. Biochim Biophys Acta 2006, I 763:690-699.

224. Huang XP, O'Brien PJ and Templeton DM: Mitochondrial involvement in genetically determined transition metal toxicity I. Iron toxicity. Chemico-Biological Interactions 2006, 163:68-76.

225. Nairz $M$ and Weiss G: Molecular and clinical aspects of iron homeostasis: from anemia to hemochromatosis. Wiener Klinische Wochenschrift 2006, I I 8:442-462.

226. Anderson GJ: Mechanisms of iron loading and toxicity. Am J Hematol 2007, 82: | |28-3|

227. Andrews NC and Schmidt PJ: Iron homeostasis. Annu Rev Physiol 2007, 69:69-85.

228. Harrison-Findik DD: Role of alcohol in the regulation of iron metabolism. World J Gastroenterol 2007, I 3:4925-30.

229. Jones BC, Beard JL, Gibson JN, Unger EL, Allen RP, McCarthy KA and Earley C]: Systems genetic analysis of peripheral iron parameters in the mouse. Am J Physiol Regul Integr Comp Physiol 2007, 293:RI I6-24.

230. Mithen R: Effect of genotype on micronutrient absorption and metabolism: a review of iron, copper, iodine and selenium, and folates. Int J Vitam Nutr Res 2007, 77:205-I6.

231. Vallerio LG: Mammalian iron metabolism. Toxicology Mechanisms and Methods 2007, I7:497-5I7.

232. Andrews NC: Forging a field: the golden age of iron biology. Blood 2008, I I 2:219-30.

233. Beard J and Han O: Systemic iron status. Biochim Biophys Acta 2008 in press.

234. Deugnier $Y$, Brissot $P$ and Loréal $O$ : Iron and the liver: update 2008. I Hepatol 2008, 48(Suppl I):SII3-23.

235. Ganz T: Iron homeostasis: fitting the puzzle pieces together. Cell Metab 2008, 7:288-90.

236. Handelman G] and Levin NW: Iron and anemia in human biology: a review of mechanisms. Heart Fail Rev 2008, 1 3:393-404.

237. Hower V, Mendes P, Torti FM, Laubenbacher R, Akman S, Shulaev V and Torti SV: A general map of iron metabolism and tissuespecific subnetworks. Mol Biosyst 2008.

238. Kemna EH, Tjalsma H, Willems HL and Swinkels DW: Hepcidin: from discovery to differential diagnosis. Haematologica 2008, 93:90-7

239. Kong $W$, Duan $X$, Shi $Z$ and Chang $Y$ : Iron metabolism in the mononuclear phagocyte system. Progr Nat Sci 2008, I 8: I 197-1202.

240. Levi $S$ and Rovida $E:$ The role of iron in mitochondrial function. Biochim Biophys Acta 2008 in press.

24l. Wrighting DM and Andrews NC: Iron homeostasis and erythropoiesis. Red Cell Development 2008, 82:14I-I67.

242. Frazer DM and Anderson GJ: Iron Imports. I. Intestinal iron absorption and its regulation. Amer J Physiol 2005, 289: G63I-G635

243. Mackenzie B and Garrick MD: Iron Imports. II. Iron uptake at the apical membrane in the intestine. American Journal of Physiology-Gastrointestinal and Liver Physiology 2005, 289:G98I-G986.

244. Wessling-Resnick M: Iron Imports. III. Transfer of iron from the mucosa into circulation. American Journal of PhysiologyGastrointestinal and Liver Physiology 2006, 290:GI-G6.

245. Ganz T and Nemeth E: Iron imports. IV. Hepcidin and regulation of body iron metabolism. Amer J Physiol 2006, 290: G199-G203.

246. Fleming RE and Britton RS: Iron Imports. VI. HFE and regulation of intestinal iron absorption. American Journal of Physiology-Gastrointestinal and Liver Physiology 2006, 290:G590-G594.

247. Ma YX, Yeh M, Yeh KY and Glass J: Iron imports. V. Transport of iron through the intestinal epithelium. American Journal of Physiology-Gastrointestinal and Liver Physiology 2006, 290:G4I7-G422.

248. De Domenico I, Ward DM and Kaplan J: Regulation of iron acquisition and storage: consequences for iron-linked disorders. Nature Reviews Molecular Cell Biology 2008, 9:72-8I.

249. Gunshin H, Mackenzie B, Berger UV, Gunshin Y, Romero MF Boron WF, Nussberger S, Gollan JL and Hediger MA: Cloning and characterization of a mammalian proton-coupled metal-ion transporter. Nature 1997, 388:482-8.

250. Forbes JR and Gros P: Divalent-metal transport by NRAMP proteins at the interface of host-pathogen interactions. Trends Microbiol 2001, 9:397-403.

25I. Shayeghi M, Latunde-Dada GO, Oakhill JS, Laftah AH, Takeuchi K, Halliday N, Khan Y, Warley A, McCann FE, Hider RC, Frazer DM,
Anderson GJ, Vulpe CD, Simpson RJ and McKie AT: Identification of an intestinal heme transporter. Cell 2005, I 22:789-80I.

252. Ryter SW, Alam J and Choi AM: Heme oxygenase-I/carbon monoxide: from basic science to therapeutic applications. Physiol Rev 2006, 86:583-650.

253. Vítek $L$ and Schwertner HA: The heme catabolic pathway and its protective effects on oxidative stress-mediated diseases. Adv Clin Chem 2007, 43: I-57.

254. Abraham NG and Kappas A: Pharmacological and clinical aspects of heme oxygenase. Pharmacol Rev 2008, 60:79-127.

255. Ponka P: Tissue-specific regulation of iron metabolism and heme synthesis: Distinct control mechanisms in erythroid cells. Blood 1997, 89: I-25.

256. Linder MC, Mortya M, Whon A, Kassa A and Gilley C: Vesicular transport of $\mathrm{Fe}$ and interaction with other metal ions in polarized Caco2 Cell monolayers. Biological Research 2006, 39: $143-156$.

257. Moriya $M$ and Linder MC: Vesicular transport and apotransferrin in intestinal iron absorption, as shown in the Caco-2 cell model. Amer J Physiol 2006, 290:G30I-G309.

258. Petrat F, de Groot H, Sustmann R and Rauen U: The chelatable iron pool in living cells: A methodically defined quantity. Biological Chemistry 2002, 383:489-502.

259. Tenopoulou M, Kurz T, Doulias PT, Galaris D and Brunk UT: Does the calcein-AM method assay the total cellular 'labile iron pool' or only a fraction of it? Biochem J 2007, 403:26I-6.

260. Shvartsman M, Kikkeri R, Shanzer A and Cabantchik Zl: Nontransferrin-bound iron reaches mitochondria by a chelatorinaccessible mechanism: biological and clinical implications. Am J Physiol Cell Physiol 2007, 293:CI383-94.

26I. Breuer W, Shvartsman $M$ and Cabantchik ZI: Intracellular labile iron. Int J Biochem Cell Biol 2008, 40:350-4

262. Fakih S, Podinovskaia $M$, Kong $X$, Collins HL, Schaible UE and Hider RC: Targeting the lysosome: fluorescent iron(III) chelators to selectively monitor endosomal/lysosomal labile iron pools. I Med Chem 2008, 5 I:4539-52.

263. Arosio P, Ingrassia $R$ and Cavadini P: Ferritins: A family of molecules for iron storage, antioxidation and more. Biochim Biophys Acta 2008.

264. Shi H, Bencze KZ, Stemmler TL and Philpott CC: A cytosolic iron chaperone that delivers iron to ferritin. Science 2008 , 320: $1207-10$

265. Hallberg L and Hulthen L: Perspectives on iron absorption. Blood Cells Molecules and Diseases 2002, 29:562-573.

266. Arosio $P$ and Levi S: Ferritin, iron homeostasis, and oxidative damage. Free Radic Biol Med 2002, 33:457-63.

267. Lee DH, Zacharski LR and Jacobs DR Jr: Comparison of the serum ferritin and percentage of transferrin saturation as exposure markers of iron-driven oxidative stress-related disease outcomes. Am Heart J 2006, I 5 I: I 247 el-7.

268. McKie AT and Barlow DJ: The SLC40 basolateral iron transporter family (IREGi/ferroportin/MTPI). Pflugers Arch 2004, 447:80I-6

269. Donovan A, Lima CA, Pinkus JL, Pinkus GS, Zon LI, Robine S and Andrews NC: The iron exporter ferroportin/SIc40al is essential for iron homeostasis. Cell Metabolism 2005, I: I91-200.

270. Ganz T: Cellular iron: ferroportin is the only way out. Cell Metab 2005, I:I55-7.

271. Oates PS: The role of hepcidin and ferroportin in iron absorption. Histol Histopathol 2007, 22:79|-804

272. Vulpe CD, Kuo YM, Murphy TL, Cowley L, Askwith C, Libina N, Gitschier J and Anderson GJ: Hephaestin, a ceruloplasmin homologue implicated in intestinal iron transport, is defective in the sla mouse. Nature Genetics 1999, 21:195-199.

273. Worwood M: Serum transferrin receptor assays and their application. Annals of Clinical Biochemistry 2002, 39:22 I-230.

274. Mense SM and Zhang L: Herne: a versatile signaling molecule controlling the activities of diverse regulators ranging from transcription factors to MAP kinases. Cell Res 2006, 16:681-692.

275. Balla J, Vercellotti GM, Nath K, Yachie A, Nagy E, Eaton JW and Balla G: Haem, haem oxygenase and ferritin in vascular endothelial cell injury. Nephrology Dialysis Transplantation 2003, 1 8:8-12.

276. Choi AM and Alam J: Heme oxygenase-I: function, regulation, and implication of a novel stress-inducible protein in oxidant-induced lung injury. Am J Respir Cell Mol Biol I996, 15:9-19.

277. Foresti R, Green CJ and Motterlini R: Generation of bile pigments by haem oxygenase: a refined cellular strategy in 
response to stressful insults. Biochem Soc Symp 2004, 71:177-192.

278. Poon HF, Calabrese V, Scapagnini G and Butterfield DA: Free radicals: key to brain aging and heme oxygenase as a cellular response to oxidative stress. J Gerontol A Biol Sci Med Sci 2004, 59:478-93.

279. Akagi R, Takahashi T and Sassa S: Cytoprotective effects of heme oxygenase in acute renal failure. Cellular Stress Responses in Renal Diseases, Contrib Nephrol 2005, 148:70-85.

280. Fredenburgh LE, Perrella MA and Mitsialis SA: The role of heme oxygenase-I in pulmonary disease. Amer J Resp Cell Mol Biol 2007, 36: 158-165.

28I. Gueler F, Park JK, Rong S, Kirsch T, Lindschau C, Zheng W, Elger M, Fiebeler A, Fliser D, Luft FC and Haller H: Statins attenuate ischemia-reperfusion injury by inducing heme oxygenase-I in infiltrating macrophages. Am J Pathol 2007, 170:1 192-1199.

282. Koizumi S: Human heme oxygenase-I deficiency: a lesson on serendipity in the discovery of the novel disease. Pediatr Int 2007, 49: 125-32.

283. Horváth I, Donnelly LE, Kiss A, Paredi P, Kharitonov SA and Barnes PJ: Raised levels of exhaled carbon monoxide are associated with an increased expression of heme oxygenase$I$ in airway macrophages in asthma: a new marker of oxidative stress. Thorax 1998, 53:668-72.

284. Uhlen M, Bjorling E, Agaton C, Szigyarto CA, Amini B, Andersen E, Andersson AC, Angelidou P, Asplund A, Asplund C, Berglund L, Bergstrom K, Brumer $H$, Cerjan D, Ekstrom M, Elobeid A, Eriksson C, Fagerberg L, Falk R, Fall J, Forsberg M, Bjorklund MG, Gumbel K, Halimi A, Hallin I, Hamsten C, Hansson M, Hedhammar M, Hercules G, Kampf C, Larsson K, Lindskog M, Lodewyckx W, Lund J, Lundeberg J, Magnusson K, Malm E, Nilsson P, Odling J, Oksvold P, Olsson I, Oster E, Ottosson J, Paavilainen L, Persson A, Rimini R, Rockberg J, Runeson M, Sivertsson A, Skollermo A, Steen J, Stenvall M, Sterky F, Stromberg S, Sundberg M, Tegel H, Tourle S, Wahlund E, Walden A, Wan J, Wernerus H, Westberg J, Wester K, Wrethagen U, Xu LL, Hober S and Ponten F: A human protein atlas for normal and cancer tissues based on antibody proteomics. Mol Cell Proteomics 2005, 4:1920-32.

285. Lee DW and Andersen JK: Role of HIF-I in iron regulation: potential therapeutic strategy for neurodegenerative disorders. Curr Mol Med 2006, 6:883-893.

286. Rouault TA: The role of iron regulatory proteins in mammalian iron homeostasis and disease. Nature Chem Biol 2006, 2:406-4I4

287. Wallander ML, Leibold EA and Eisenstein RS: Molecular control of vertebrate iron homeostasis by iron regulatory proteins. Biochim Biophys Acta 2006, 1763:668-689.

288. Lee K, Roth RA and LaPres JJ: Hypoxia, drug therapy and toxicity. Pharmacology \& Therapeutics 2007, I | 3:229-246.

289. Pigeon C, llyin G, Courselaud B, Leroyer P, Turlin B, Brissot P and Loreal O: A new mouse liver-specific gene, encoding a protein homologous to human antimicrobial peptide hepcidin, is overexpressed during iron overload. J Biol Chem 200I, 276:78||-78|9.

290. Fleming RE and Sly WS: Hepcidin: A putative iron-regulatory hormone relevant to hereditary hemochromatosis and the anemia of chronic disease. Proc Nal Acad Sci 200I, 98:8I60-8I62.

291. Nicolas G, Bennoun M, Devaux I, Beaumont C, Grandchamp B, Kahn $A$ and Vaulont S: Lack of hepcidin gene expression and severe tissue iron overload in upstream stimulatory factor 2 (USF2) knockout mice. Proc Natl Acad Sci 200I, 98:8780-8785.

292. Weinstein DA, Roy CN, Fleming MD, Loda MF, Wolfsdorf JI and Andrews NC: Inappropriate expression of hepcidin is associated with iron refractory anemia: implications for the anemia of chronic disease. Blood 2002, 100:3776-81.

293. Bridle KR, Frazer DM, Wilkins SJ, Dixon JL, Purdie DM, Crawford DHG, Subramaniam VN, Powell LW, Anderson G] and Ramm GA: Disrupted hepcidin regulation in HFE-associated haemochromatosis and the liver as a regulator of body iron homoeostasis. Lancet 2003, 36 1:669-673.

294. Nicolas G, Viatte L, Lou DQ, Bennoun M, Beaumont C, Kahn A, Andrews NC and Vaulont S: Constitutive hepcidin expression prevents iron overload in a mouse model of hemochromatosis. Nature Genetics 2003, 34:97-I0I.

295. Roetto A, Papanikolaou G, Politou M, Alberti F, Girelli D, Christakis J, Loukopoulos D and Camaschella C: Mutant antimicrobial peptide hepcidin is associated with severe juvenile hemochromatosis. Nature Genetics 2003, 33:21-22.
296. Deicher R and Horl WH: Hepcidin: a molecular link between inflammation and anaemia. Nephrol Dial Transplant 2004, 19:521-4.

297. Leong WI and Lönnerdal B: Hepcidin, the recently identified peptide that appears to regulate iron absorption. J Nutr 2004, 134: $1-4$.

298. Robson KJ: Hepcidin and its role in iron absorption. Gut 2004, 53:617-9.

299. Camaschella C: Understanding iron homeostasis through genetic analysis of hemochromatosis and related disorders. Blood 2005, 106:3710-7.

300. Loréal O, Haziza-Pigeon C, Troadec MB, Detivaud L, Turlin B, Courselaud B, llyin G and Brissot P: Hepcidin in iron metabolism. Curr Protein Pept Sci 2005, 6:279-9I.

30I. Roetto A and Camaschella C: New insights into iron homeostasis through the study of non-HFE hereditary haemochromatosis. Best Pract Res Clin Haematol 2005, 1 8:235-50.

302. Roy CN and Andrews NC: Anemia of inflammation: the hepcidin link. Curr Opin Hematol 2005, I 2:107-II.

303. Beutler E: Hemochromatosis: Genetics and pathophysiology. Annu Rev Med 2006, 57:33I-347.

304. Deicher R and Hörl WH: New insights into the regulation of iron homeostasis. Eur J Clin Invest 2006, 36:30I-309.

305. Farnaud S, Patel A and Evans RW: Modelling of a metalcontaining hepcidin. Biometals 2006, 19:527-533.

306. Ganz T: Hepcidin - A peptide hormone at the interface of innate immunity and iron metabolism. Antimicrobial Peptides and Human Disease, Current Topics in Microbiology and Immunology 2006, 306: 183-198.

307. Harrison-Findik DD, Schafer D, Klein E, Timchenko NA, Kulaksiz H, Clemens D, Fein E, Andriopoulos B, Pantopoulos K and Gollan I: Alcohol metabolism-mediated oxidative stress down-regulates hepcidin transcription and leads to increased duodenal iron transporter expression. J Biol Chem 2006, 28I:22974-22982.

308. Hugman A: Hepcidin: an important new regulator of iron homeostasis. Clin Lab Haematol 2006, 28:75-83.

309. Mena NP, Esparza AL and Núñez MT: Regulation of transepithelial transport of iron by hepcidin. Biol Res 2006, 39:19I-193.

310. Mok H, Mlodnicka AE, Hentze MW, Muckenthaler $M$ and Schumacher A: The molecular circuitry regulating the switch between iron deficiency and overload in mice. J Biol Chem 2006, 28I:7946-5I.

3II. Nemeth E and Ganz T: Regulation of iron metabolism by hepcidin. Annual Review of Nutrition 2006, 26:323-342.

312. Atanasiu V, Manolescu B and Stoian I: Hepcidin - central regulator of iron metabolism. European Journal of Haematology 2007, 78: $1-10$.

313. Malyszko J and Mysliwiec M: Hepcidin in anemia and inflammation in chronic kidney disease. Kidney \& Blood Pressure Research 2007, 30: I5-30.

314. Oates PS and Ahmed U: Molecular regulation of hepatic expression of iron regulatory hormone hepcidin. J Gastroenterol Hepatol 2007, 22:1378-87.

315. Barisani D, Pelucchi S, Mariani R, Galimberti S, Trombini P, Fumagalli D, Meneveri R, Nemeth E, Ganz T and Piperno A: Hepcidin and iron-related gene expression in subjects with Dysmetabolic Hepatic Iron Overload. J Hepatol 2008, 49: $123-33$

316. Camaschella $C$ and Silvestri L: New and old players in the hepcidin pathway. Haematologica 2008, 93:|44|-4

317. De Domenico I, Nemeth E, Nelson JM, Phillips JD, Ajioka RS, Kay MS, Kushner JP, Ganz T, Ward DM and Kaplan J: The hepcidinbinding site on ferroportin is evolutionarily conserved. Cell Metab 2008, 8: 146-56.

318. Farnaud S, Rapisarda C, Bui T, Drake A, Cammack R and Evans RW: Identification of an iron-hepcidin complex. Biochem J 2008, 413:553-7.

319. Kartikasari AE, Roelofs R, Schaeps RM, Kemna EH, Peters WH, Swinkels DW and Tjalsma $H$ : Secretion of bioactive hepcidin-25 by liver cells correlates with its gene transcription and points towards synergism between iron and inflammation signaling pathways. Biochim Biophys Acta 2008, I 784:2029-37.

320. Muckenthaler MU, Galy B and Hentze MW: Systemic iron homeostasis and the iron-responsive element/iron-regulatory protein (IRE/IRP) regulatory network. Annu Rev Nutr 2008, 28:197-2I3.

321. Nemeth E: Iron regulation and erythropoiesis. Curr Opin Hematol 2008, 15:169-75. 
322. Nishina S, Hino K, Korenaga M, Vecchi C, Pietrangelo A, Mizukami Y, Furutani T, Sakai A, Okuda M, Hidaka I, Okita K and Sakaida I: Hepatitis $C$ virus-induced reactive oxygen species raise hepatic iron level in mice by reducing hepcidin transcription. Gastroenterology 2008, I34:226-38.

323. Peeling $P$, Dawson B, Goodman C, Landers $G$ and Trinder D: Athletic induced iron deficiency: new insights into the role of inflammation, cytokines and hormones. Eur J Appl Physiol 2008, I03:38I-9I.

324. Swinkels DW, Girelli D, Laarakkers C, Kroot J, Campostrini N, Kemna $\mathrm{EH}$ and Tjalsma $\mathrm{H}$ : Advances in quantitative hepcidin measurements by time-of-flight mass spectrometry. PLoS ONE 2008, 3:e2706.

325. Swinkels DW and Wetzels JF: Hepcidin: a new tool in the management of anaemia in patients with chronic kidney disease? Nephrol Dial Transplant 2008, 23:2450-3.

326. Toblli JE, Cao G, Rivas C and Kulaksiz H: Heart and iron deficiency anaemia in rats with renal insufficiency: The role of hepcidin. Nephrology (Carlton) 2008, I 3:636-645.

327. Trinder D, Ayonrinde OT and Olynyk JK: HCV, iron, and oxidative stress: the new choreography of hepcidin. Gastroenterology 2008, I34:348-5I.

328. Knutson MD, Oukka M, Koss LM, Aydemir F and WesslingResnick M: Iron release from macrophages after erythrophagocytosis is up-regulated by ferroportin I overexpression and down-regulated by hepcidin. Proc Natl Acad Sci USA 2005, 102: 1324-8.

329. Nemeth E, Tuttle MS, Powelson J, Vaughn MB, Donovan A, Ward DM, Ganz T and Kaplan J: Hepcidin regulates cellular iron efflux by binding to ferroportin and inducing its internalization. Science 2004, 306:2090-2093.

330. Delaby C, Pilard N, Goncalves AS, Beaumont C and CanonneHergaux F: Presence of the iron exporter ferroportin at the plasma membrane of macrophages is enhanced by iron loading and down-regulated by hepcidin. Blood 2005, I06:3979-84.

331. Ganz T: Hepcidin - a regulator of intestinal iron absorption and iron recycling by macrophages. Best Practice \& Research Clinical Haematology 2005, I 8: I7I-182.

332. Papanikolaou G, Tzilianos M, Christakis Jl, Bogdanos D, Tsimirika K, MacFarlane J, Goldberg YP, Sakellaropoulos N, Ganz T and Nemeth E: Hepcidin in iron overload disorders. Blood 2005, 105:4103-4105.

333. Lesbordes-Brion IC, Viatte L, Bennoun M, Lou DO, Ramey G, Houbron C, Hamard G, Kahn A and Vaulont S: Targeted disruption of the hepcidin I gene results in severe hemochromatosis. Blood 2006, I08:|402-|405.

334. Anderson GJ and Frazer DM: Iron metabolism meets signal transduction. Nature Genetics 2006, 38:503-504.

335. Kulaksiz H, Gehrke SG, Janetzko A, Rost D, Bruckner T, Kallinowski B and Stremmel W: Pro-hepcidin: expression and cell specific localisation in the liver and its regulation in hereditary haemochromatosis, chronic renal insufficiency, and renal anaemia. Gut 2004, 53:735-743.

336. Kulaksiz H, Theilig F, Bachmann S, Gehrke SG, Rost D, Janetzko A, Cetin $Y$ and Stremmel $W$ : The iron-regulatory peptide hormone hepcidin: expression and cellular localization in the mammalian kidney. Journal of Endocrinology 2005, I 84:36I-370.

337. Tiker F, Celik B, Tarcan A, Kilicdag H, Ozbek N and Gurakan B: Serum pro-hepcidin levels and relationships with iron parameters in healthy preterm and term newborns. Pediatr Hematol Oncol 2006, 23:293-297.

338. Roe MA, Spinks C, Heath ALM, Harvey LJ, Foxall R, Wimperis J, Wolf $C$ and Fairweather-Tait SJ: Serum prohepcidin concentration: no association with iron absorption in healthy men; and no relationship with iron status in men carrying. HFE mutations, hereditary haemochromatosis patients undergoing phlebotomy treatment, or pregnant women. $\mathrm{Br} J \mathrm{Nutr}$ 2007, 97:544-549.

339. Nicolas G, Chauvet C, Viatte L, Danan JL, Bigard X, Devaux I, Beaumont $C$, Kahn $A$ and Vaulont $S$ : The gene encoding the iron regulatory peptide hepcidin is regulated by anemia, hypoxia, and inflammation. I Clin Invest 2002, I I 0:1037-1044.

340. Pak M, Lopez MA, Gabayan V, Ganz T and Rivera S: Suppression of hepcidin during anemia requires erythropoietic activity. Blood 2006, 108:3730-5.

34I. Nemeth E, Valore EV, Territo M, Schiller G, Lichtenstein A and Ganz T: Hepcidin, a putative mediator of anemia of inflammation, is a type II acute-phase protein. Blood 2003, I 0 I:246I-2463.

342. Nemeth E, Rivera S, Gabayan V, Keller C, Taudorf S, Pedersen BK and Ganz T: IL-6 mediates hypoferremia of inflammation by inducing the synthesis of the iron regulatory hormone hepcidin. J Clin Invest 2004, I I 3: I 27 I-I 276.

343. Kemna E, Pickkers P, Nemeth E, Hoeven van der $H$ and Swinkels D: Time-course analysis of hepcidin, serum iron, and plasma cytokine levels in humans injected with LPS. Blood 2005, 106: | 864-1866.

344. Fleming RE: Iron and inflammation: cross-talk between pathways regulating hepcidin. J Mol Med 2008, 86:49I-4.

345. Malyszko J, Malyszko JS, Pawlak K, Drozdowska-Rams L, Brzosko S and Mysliwiec M: Hepcidin is linked to anemia and inflammation in peritoneal dialysis patients. Perit Dial Int 2008, 28:4I8-2I.

346. Wang RH, Li CL, Xu XL, Zheng Y, Xiao CY, Zerfas P, Cooperman S, Eckhaus $M$, Rouault T, Mishra $L$ and Deng $C X$ : A role of SMAD4 in iron metabolism through the positive regulation of hepicidin expression. Cell Metabolism 2005, 2:399-409.

347. Falzacappa MVV, Spasic MV, Kessler R, Stolte J, Hentze MW and Muckenthaler MU: STAT3 mediates hepatic hepcidin expression and its inflammatory stimulation. Blood 2007, 109:353-358.

348. Pietrangelo A, Dierssen U, Valli L, Garuti C, Rump A, Corradini E, Ernst $M$, Klein $C$ and Trautwein C: STAT3 is required for IL-6gp I30-dependent activation of hepcidin in vivo. Gastroenterol 2007, 132:294-300.

349. Choi SO, Cho YS, Kim HL and Park JW: ROS mediate the hypoxic repression of the hepcidin gene by inhibiting C/EBP alpha and STAT-3. Biochem Biophys Res Comm 2007, 356:3 I2-3 I 7.

350. Flanagan JM, Truksa J, Peng HF, Lee $P$ and Beutler E: In vivo imaging of hepcidin promoter stimulation by iron and inflammation. Blood Cells Mols Dis 2007, 38:253-257.

35I. Détivaud L, Nemeth E, Boudjema K, Turlin B, Troadec MB, Leroyer P, Ropert M, Jacquelinet S, Courselaud B, Ganz T, Brissot $P$ and Loréal $O$ : Hepcidin levels in humans are correlated with hepatic iron stores, hemoglobin levels, and hepatic function. Blood 2005, 106:746-748.

352. Weizer-Stern O, Adamsky K, Amariglio N, Rachmilewitz E, Breda L, Rivella $S$ and Rechavi G: mRNA expression of iron regulatory genes in beta-thalassemia intermedia and beta-thalassemia major mouse models. Amer J Hematol 2006, 81:479-483.

353. Weizer-Stern O, Adamsky K, Amariglio N, Levin C, Koren A, Breuer W, Rachmilewitz E, Breda L, Rivella S, Cabantchik ZI and Rechavi G: Downregulation of hepcidin and haemojuvelin expression in the hepatocyte cell-line HepG2 induced by thalassaemic sera. $\mathrm{Br} J$ Haematol 2006, I35:I29-I38.

354. Weizer-Stern O, Adamsky K, Margalit O, Ashur-Fabian O, Givol D, Amariglio $\mathrm{N}$ and Rechavi G: Hepcidin, a key regulator of iron metabolism, is transcriptionally activated by p53. $\mathrm{Br} J$ Haematol 2007, 138:253-62.

355. Sullivan JL: Macrophage iron, hepcidin, and atherosclerotic plaque stability. Exp Biol Med (Maywood) 2007, 232:1014-20.

356. Adamsky K, Weizer O, Amariglio N, Breda L, Harmelin A, Rivella S, Rachmilewitz $E$ and Rechavi G: Decreased hepcidin mRNA expression in thalassemic mice. $\mathrm{Br} J$ Haematol 2004, 124:123-124.

357. Flower DR, North AC and Sansom CE: The lipocalin protein family: structural and sequence overview. Biochim Biophys Acta 2000, I 482:9-24.

358. Kjeldsen L, Johnsen $A H$, Sengelov $H$ and Borregaard $N$ : Isolation and primary structure of NGAL, a novel protein associated with human neutrophil gelatinase. J Biol Chem 1993, 268: 10425-32.

359. Stoesz SP and Gould MN: Overexpression of neu-related lipocalin (NRL) in neu-initiated but not ras or chemically initiated rat mammary carcinomas. Oncogene 1995, I I:2233-4l.

360. Kjeldsen L, Cowland JB and Borregaard N: Human neutrophil gelatinase-associated lipocalin and homologous proteins in rat and mouse. Biochim Biophys Acta 2000, I 482:272-83.

361. Liu Q, Ryon J and Nilsen-Hamilton M: Uterocalin: a mouse acute phase protein expressed in the uterus around birth. Mol Reprod Dev 1997, 46:507-14.

362. Goetz DH, Holmes MA, Borregaard N, Bluhm ME, Raymond KN and Strong RK: The neutrophil lipocalin NGAL is a bacteriostatic agent that interferes with siderophore-mediated iron acquisition. Mol Cell 2002, I 0: I033-43. 
363. Flo TH, Smith KD, Sato S, Rodriguez DJ, Holmes MA, Strong RK, Akira $S$ and Aderem A: Lipocalin 2 mediates an innate immune response to bacterial infection by sequestrating iron. Nature 2004, 432:9|7-2I.

364. Fluckinger $M$, Haas $H$, Merschak $P$, Glasgow BJ and Redl B: Human tear lipocalin exhibits antimicrobial activity by scavenging microbial siderophores. Antimicrob Agents Chemother 2004, 48:3367-72.

365. Holmes MA, Paulsene W, jide $X$, Ratledge $C$ and Strong RK: Siderocalin (Lcn 2) also binds carboxymycobactins, potentially defending against mycobacterial infections through iron sequestration. Structure 2005, | 3:29-4I.

366. Berger T, Togawa A, Duncan GS, Elia AJ, You-Ten A, Wakeham A, Fong HE, Cheung CC and Mak TW: Lipocalin 2-deficient mice exhibit increased sensitivity to Escherichia coli infection but not to ischemia-reperfusion injury. Proc Natl Acad Sci USA 2006, 103:1834-9.

367. Borregaard $\mathrm{N}$ and Cowland JB: Neutrophil gelatinase-associated lipocalin, a siderophore-binding eukaryotic protein. Biometals 2006, I9:2III-2I5.

368. Yang J, Goetz D, Li JY, Wang W, Mori K, Setlik D, Du T, ErdjumentBromage $\mathrm{H}$, Tempst $\mathrm{P}$, Strong $\mathrm{R}$ and Barasch J: An iron delivery pathway mediated by a lipocalin. Mol Cell 2002, I 0:1045-56.

369. Coles M, Diercks T, Muehlenweg B, Bartsch S, Zolzer V, Tschesche $H$ and Kessler $H$ : The solution structure and dynamics of human neutrophil gelatinase-associated lipocalin. J Mol Biol 1999, 289:139-57.

370. Tschesche H, Zolzer V, Triebel S and Bartsch S: The human neutrophil lipocalin supports the allosteric activation of matrix metalloproteinases. Eur J Biochem 200I, 268:1918-1928.

37I. Yan L, Borregaard N, Kjeldsen $L$ and Moses MA: The high molecular weight urinary matrix metalloproteinase (MMP) activity is a complex of gelatinase B/MMP-9 and neutrophil gelatinase-associated lipocalin (NGAL). Modulation of MMP-9 activity by NGAL. J Biol Chem 200I, 276:37258-65.

372. Devarajan P, Mishra J, Supavekin S, Patterson LT and Steven Potter S: Gene expression in early ischemic renal injury: clues towards pathogenesis, biomarker discovery, and novel therapeutics. Mol Genet Metab 2003, 80:365-76.

373. Mishra J, Ma Q, Prada A, Mitsnefes M, Zahedi K, Yang J, Barasch J and Devarajan P: Identification of neutrophil gelatinase-associated lipocalin as a novel early urinary biomarker for ischemic renal injury. I Am Soc Nephrol 2003, I 4:2534-43.

374. Mishra J, Dent C, Tarabishi R, Mitsnefes MM, Ma Q, Kelly C, Ruff SM, Zahedi K, Shao M, Bean J, Mori K, Barasch J and Devarajan P: Neutrophil gelatinase-associated lipocalin (NGAL) as a biomarker for acute renal injury after cardiac surgery. Lancet 2005, 365: $|23|-8$.

375. Mori K, Lee HT, Rapoport D, Drexler IR, Foster K, Yang J, SchmidtOtt KM, Chen X, Li JY, Weiss S, Mishra J, Cheema FH, Markowitz G, Suganami T, Sawai K, Mukoyama M, Kunis C, D'Agati V, Devarajan P and Barasch J: Endocytic delivery of lipoccalin-siderophoreiron complex rescues the kidney from ischemia-reperfusion injury. Journal of Clinical Investigation 2005, I I 5:6 I 0-62 I.

376. Herget-Rosenthal S: One step forward in the early detection of acute renal failure. Lancet 2005, 365:I205-I206.

377. Trachtman H, Christen E, Cnaan A, Patrick J, Mai V, Mishra J, Jain A, Bullington $N$ and Devarajan $P$ : Urinary neutrophil gelatinaseassociated lipocalcin in D+HUS: a novel marker of renal injury. Pediatric Nephrology 2006, 21 1:989-994.

378. Dent CL, Ma O, Dastrala S, Bennett M, Mitsnefes MM, Barasch J and Devarajan P: Plasma neutrophil gelatinase-associated lipocalin predicts acute kidney injury, morbidity and mortality after pediatric cardiac surgery: a prospective uncontrolled cohort study. Critical Care 2007, I I:RI 27.

379. Devarajan P: Update on mechanisms of ischemic acute kidney injury. Journal of the American Society of Nephrology 2006, I 7:1503-1520.

380. Schmidt-Ott KM, Mori K, Kalandadze A, Li JY, Paragas N, Nicholas T, Devarajan $P$ and Barasch J: Neutrophil gelatinase-associated lipocalin-mediated iron traffic in kidney epithelia. Curr Opin Nephrol Hypertens 2006, I 5:442-9.

381. Schmidt-Ott KM, Mori K, Li JY, Kalandadze A, Cohen DJ, Devarajan $P$ and Barasch J: Dual action of neutrophil gelatinase-associated lipocalin. J Am Soc Nephrol 2007, I 8:407-I3.

382. Mori $\mathrm{K}$ and Nakao K: Neutrophil gelatinase-associated lipocalin as the real-time indicator of active kidney damage. Kidney Int 2007, 71:967-70.

383. Mitsnefes MM, Kathman TS, Mishra J, Kartal J, Khoury PR, Nickolas TL, Barasch J and Devarajan P: Serum neutrophil gelatinase-associated lipocalin as a marker of renal function in children with chronic kidney disease. Pediatr Nephrol 2007 22: $101-8$.

384. Zappitelli M, Washburn KK, Arikan AA, Loftis L, Ma Q, Devarajan P, Parikh CR and Goldstein SL: Urine neutrophil gelatinaseassociated lipocalin is an early marker of acute kidney injury in critically ill children: a prospective cohort study. Critical Care 2007, I I:

385. Molitoris BA, Melnikov VY, Okusa MD and Himmelfarb J: Technology Insight: biomarker development in acute kidney injurywhat can we anticipate? Nat Clin Pract Nephrol 2008, 4:I54-65.

386. Nickolas TL, O'Rourke MJ, Yang J, Sise ME, Canetta PA, Barasch N, Buchen C, Khan F, Mori K, Giglio J, Devarajan P and Barasch J: Sensitivity and specificity of a single emergency department measurement of urinary neutrophil gelatinase-associated lipocalin for diagnosing acute kidney injury. Ann Intern Med 2008, I 48:810-9.

387. Malyszko J, Bachorzewska-Gajewska H, Sitniewska E, Malyszko JS, Poniatowski B and Dobrzycki S: Serum neutrophil gelatinaseassociated lipocalin as a marker of renal function in nondiabetic patients with stage 2-4 chronic kidney disease. Ren Fail 2008, 30:625-8.

388. Devarajan P: NGAL in acute kidney injury: from serendipity to utility. Am J Kidney Dis 2008, 52:395-9.

389. Devarajan P: Neutrophil gelatinase-associated lipocalin (NGAL): a new marker of kidney disease. Scand J Clin Lab Invest Suppl 2008, 241:89-94.

390. Devireddy LR, Gazin C, Zhu X and Green MR: A cell-surface receptor for lipocalin $24 \mathrm{p} 3$ selectively mediates apoptosis and iron uptake. Cell 2005, I 23: I 293-305.

391. Richardson DR: $24 \mathrm{p} 3$ and its receptor: dawn of a new iron age? Cell 2005, I 23: I 175-7.

392. Hvidberg V, Jacobsen C, Strong RK, Cowland JB, Moestrup SK and Borregaard $\mathrm{N}$ : The endocytic receptor megalin binds the iron transporting neutrophil-gelatinase-associated lipocalin with high affinity and mediates its cellular uptake. FEBS Lett 2005, 579:773-7.

393. Roudkenar MH, Kuwahara Y, Baba T, Roushandeh AM, Ebishima S Abe $S$, Ohkubo $Y$ and Fukumoto $M$ : Oxidative stress induced lipocalin 2 gene expression: Addressing its expression under the harmful conditions. Journal of Radiation Research 2007, 48:39-44.

394. Roudkenar MH, Halabian R, Ghasemipour Z, Roushandeh AM, Rouhbakhsh $M$, Nekogoftar $M$, Kuwahara $Y$, Fukumoto $M$ and Shokrgozar MA: Neutrophil Gelatinase-associated Lipocalin acts as a protective factor against $\mathbf{H}_{\mathbf{2}} \mathbf{O}_{\mathbf{2}}$ toxicity. Arch Med Res 2008, 39:560-6.

395. Gueler F, Gwinner W, Schwarz A and Haller H: Long-term effects of acute ischemia and reperfusion injury. Kidney International 2004, 66:523-527.

396. Sikorski EM, Hock T, Hill-Kapturczak N and Agarwal A: The story so far: molecular regulation of the heme oxygenase-I gene in renal injury. Am J Physiol 2004, 286:F425-F44I.

397. Shah S: Role of iron in progressive renal disease. Am J Kidney Dis 200I, 37:S30-3.

398. Aigner F, Maier HT, Schwelberger HG, Wallnofer EA, Amberger A, Obrist $P$, Berger T, Mak TW, Maglione M, Margreiter R, Schneeberger $S$ and Troppmair J: Lipocalin-2 regulates the inflammatory response during ischemia and reperfusion of the transplanted heart. Amer J Transplantation 2007, 7:779-788.

399. Abergel RJ, Moore EG, Strong RK and Raymond KN: Microbial evasion of the immune system: structural modifications of enterobactin impair siderocalin recognition. J Am Chem Soc 2006, 128:10998-9.

400. Fischbach MA, Lin H, Zhou L, Yu Y, Abergel RJ, Liu DR, Raymond KN, Wanner BL, Strong RK, Walsh CT, Aderem A and Smith KD: The pathogen-associated iroA gene cluster mediates bacterial evasion of lipocalin 2. Proc Natl Acad Sci USA 2006, I03: 16502-7.

40I. Abergel RJ, Clifton MC, Pizarro JC, Warner JA, Shuh DK, Strong RK and Raymond KN: The siderocalin/enterobactin interaction: a link between mammalian immunity and bacterial iron transport. J Am Chem Soc 2008, I30: I I524-34.

402. Cowland JB and Borregaard N: Molecular characterization and pattern of tissue expression of the gene for neutrophil gelatinase-associated lipocalin from humans. Genomics 1997 45: $17-23$

403. Xu S and Venge P: Lipocalins as biochemical markers of disease. Biochim Biophys Acta 2000, I 482:298-307. 
404. Cowland JB, Sorensen OE, Sehested $M$ and Borregaard $N$ : Neutrophil gelatinase-associated lipocalin is up-regulated in human epithelial cells by IL-I beta, but not by TNF-alpha. J Immunol 2003, I 7 I:6630-9.

405. Cowland JB, Muta T and Borregaard N: IL-Ibeta-specific upregulation of neutrophil gelatinase-associated lipocalin is controlled by IkappaB-zeta. J Immunol 2006, I 76:5559-66.

406. Elneihoum AM, Falke $P$, Axelsson L, Lundberg $E$, Lindgarde $F$ and Ohlsson K: Leukocyte activation detected by increased plasma levels of inflammatory mediators in patients with ischemic cerebrovascular diseases. Stroke 1996, 27:1734-8.

407. Elneihoum AM, Falke $P$, Hedblad B, Lindgarde F and Ohlsson $K$ : Leukocyte activation in atherosclerosis: correlation with risk factors. Atherosclerosis 1997, I 3 I:79-84.

408. Falke $\mathrm{P}$, Elneihoum $\mathrm{AM}$ and Ohlsson $\mathrm{K}$ : Leukocyte activation: relation to cardiovascular mortality after cerebrovascular ischemia. Cerebrovasc Dis 2000, 10:97-101.

409. Hemdahl AL, Gabrielsen A, Zhu C, Eriksson P, Hedin U, Kastrup J, Thoren P and Hansson GK: Expression of neutrophil gelatinaseassociated lipocalin in atherosclerosis and myocardial infarction. Arterioscler Thromb Vasc Biol 2006, 26: I 36-42.

410. Stoesz SP, Friedl A, Haag JD, Lindstrom MJ, Clark GM and Gould MN: Heterogeneous expression of the lipocalin NGAL in primary breast cancers. Int J Cancer 1998, 79:565-72.

4II. Tong Z, Wu X, Ovcharenko D, Zhu J, Chen CS and Kehrer JP. Neutrophil gelatinase-associated lipocalin as a survival factor. Biochem J 2005, 39I:44I-8.

412. Tong Z, Kunnumakkara AB, Wang H, Matsuo $Y$, Diagaradjane $P$ Harikumar KB, Ramachandran V, Sung B, Chakraborty A Bresalier RS, Logsdon C, Aggarwal BB, Krishnan S and Guha S: Neutrophil gelatinase-associated lipocalin: a novel suppressor of invasion and angiogenesis in pancreatic cancer. Cancer Res 2008, 68:6100-8.

413. Weinberg ED: Iron out-of-balance: a risk factor for acute and chronic diseases. Hemoglobin 2008, 32: I I7-22.

414. CESDI: Confidential Enquiry into Stillbirths and Deaths in Infancy. 5th Annual Report Maternal and Child Health Research Consortium; 1998.

4I5. LaMarca BD, Gilbert J and Granger JP: Recent progress toward the understanding of the pathophysiology of hypertension during preeclampsia. Hypertension 2008, 51:982-8.

4I6. Redman CW and Sargent IL: Placental debris, oxidative stress and pre-eclampsia. Placenta 2000, $21: 597-602$.

417. DiFederico E, Genbacev $O$ and Fisher SJ: Preeclampsia is associated with widespread apoptosis of placental cytotrophoblasts within the uterine wall. Am J Pathol 1999, | 55:293-301.

418. Leung DN, Smith SC, To KF, Sahota DS and Baker PN: Increased placental apoptosis in pregnancies complicated by preeclampsia. Am J Obstet Gynecol 200 I, I84:1249-50.

419. Huppertz B and Kingdom JC: Apoptosis in the trophoblast-role of apoptosis in placental morphogenesis. J Soc Gynecol Investig 2004, I I:353-62.

420. Harris LK, Keogh RJ, Wareing M, Baker PN, Cartwright JE, Aplin JD and Whitley GS: Invasive trophoblasts stimulate vascular smooth muscle cell apoptosis by a fas ligand-dependent mechanism. Am J Pathol 2006, 169:1863-74.

42I. Heazell AE, Moll Sj, Jones CJ, Baker PN and Crocker IP: Formation of syncytial knots is increased by hyperoxia, hypoxia and reactive oxygen species. Placenta 2007, 28(Suppl A):S33-40.

422. Keogh RJ, Harris LK, Freeman A, Baker PN, Aplin JD, Whitley GS and Cartwright JE: Fetal-derived trophoblast use the apoptotic cytokine tumor necrosis factor-alpha-related apoptosisinducing ligand to induce smooth muscle cell death. Circ Res 2007, 100:834-41.

423. Heazell AE, Lacey HA, Jones CJ, Huppertz B, Baker PN and Crocker IP: Effects of oxygen on cell turnover and expression of regulators of apoptosis in human placental trophoblast. Placenta 2008, 29:175-86.

424. Combs CA, Katz MA, Kitzmiller JL and Brescia RJ: Experimental preeclampsia produced by chronic constriction of the lower aorta: validation with longitudinal blood pressure measurements in conscious rhesus monkeys. Am J Obstet Gynecol 1993, 169:215-23.

425. Wisdom SJ, Wilson R, McKillop JH and Walker Jl: Antioxidant systems in normal pregnancy and in pregnancy-induced hypertension. Am J Obs Gynecol I99I, I65:I70I-I704.

426. Genbacev $\mathrm{O}$, Joslin $\mathrm{R}$, Damsky $\mathrm{CH}$, Polliotti $\mathrm{BM}$ and Fisher SJ: Hypoxia alters early gestation human cytotrophoblast differentiation/invasion in vitro and models the placental defects that occur in preeclampsia. I Clin Invest 1996 , 97:540-50.

427. Benyo DF, Miles TM and Conrad KP: Hypoxia stimulates cytokine production by villous explants from the human placenta. J Clin Endocrinol Metab 1997, 82:I582-8.

428. Conrad KP and Benyo DF: Placental cytokines and the pathogenesis of preeclampsia. Am J Reprod Immunol 1997, 37:240-9.

429. Lefevre G, Berkane N, Uzan S and Etienne J: Preeclampsia and oxygen free radicals. Annales De Biologie Clinique 1997, 55:443-450.

430. Conrad KP, Miles TM and Benyo DF: Circulating levels of immunoreactive cytokines in women with preeclampsia. Am J Reprod Immunol 1998, 40:102-II.

431. Davidge ST: Oxidative stress and altered endothelial cell function in preeclampsia. Semin Reprod Endocrinol 1998, 16:65-73

432. Mise H, Sagawa N, Matsumoto T, Yura S, Nanno H, Itoh H, Mori T, Masuzaki H, Hosoda K, Ogawa $Y$ and Nakao K: Augmented placental production of leptin in preeclampsia: possible involvement of placental hypoxia. J Clin Endocrinol Metab I998, 83:3225-9.

433. Walker J]: Antioxidants and inflammatory cell response in preeclampsia. Semin Reprod Endocrinol 1998, 16:47-55.

434. Walsh SW: Maternal-placental interactions of oxidative stress and antioxidants in preeclampsia. Seminars in Reproductive Endocrinology 1998, 16:93-104

435. Caniggia I, Grisaru-Gravnosky S, Kuliszewsky M, Post M and Lye S]: Inhibition of TGF-beta 3 restores the invasive capability of extravillous trophoblasts in preeclamptic pregnancies. J Clin Invest 1999, 103:164I-50.

436. Hubel CA: Oxidative stress in the pathogenesis of preeclampsia. Proc Soc Exp Biol Med 1999, 222:222-35.

437. Rinehart BK, Terrone DA, Lagoo-Deenadayalan S, Barber WH, Hale EA, Martin JN Jr and Bennett WA: Expression of the placental cytokines tumor necrosis factor alpha, interleukin I beta, and interleukin 10 is increased in preeclampsia. Am J Obstet Gynecol 1999, 181:915-20.

438. Caniggia I, Mostachfi H, Winter J, Gassmann M, Lye SJ, Kuliszewski M and Post M: Hypoxia-inducible factor-I mediates the biological effects of oxygen on human trophoblast differentiation through TGFbeta(3). J Clin Invest 2000, I 05:577-87.

439. Kharb S: Total free radical trapping antioxidant potential in pre-eclampsia. Int J Gynaecol Obstet 2000, 69:23-6.

440. Tálosi G, Endreffy E, Túri S and Németh I: Molecular and genetic aspects of preeclampsia: State of the art. Mol Genet Metab $2000,71: 565-572$

44I. Walsh SW, Vaughan JE, Wang $Y$ and Roberts LJ 2nd: Placental isoprostane is significantly increased in preeclampsia. FASEB 2000, I4:1289-96

442. Wolf M, Kettyle E, Sandler L, Ecker JL, Roberts J and Thadhani R: Obesity and preeclampsia: the potential role of inflammation. Obstet Gynecol 2001, 98:757-62

443. Benyo DF, Smarason A, Redman CW Sims C and Conrad KP. Expression of inflammatory cytokines in placentas from women with preeclampsia. J Clin Endocrinol Metab 2001, 86:2505-12.

444. Hung $\mathrm{TH}$, Skepper $\mathrm{JN}$ and Burton $\mathrm{GJ}$ : In vitro ischemiareperfusion injury in term human placenta as a model for oxidative stress in pathological pregnancies. Am J Pathol $200 \mathrm{I}$, | 59: $103 \mid-43$.

445. López-Jaramillo P, Casas JP and Serrano N: Preeclampsia: from epidemiological observations to molecular mechanisms. Braz J Med Biol Res 200I, 34: 1227-35.

446. Malek A, Sager R and Schneider H: Effect of hypoxia, oxidative stress and lipopolysaccharides on the release of prostaglandins and cytokines from human term placental explants. Placenta 2001, 22(Suppl A):S45-50.

447. Gitto E, Reiter RJ, Karbownik M, Tan DX, Gitto P, Barberi S and Barberi I: Causes of oxidative stress in the pre- and perinatal period. Biology of the Neonate 2002, 8|:|46-|57.

448. Granger JP, Alexander BT, Llinas MT, Bennett WA and Khalil RA: Pathophysiology of preeclampsia: linking placental ische$\mathrm{mia} / \mathrm{hypoxia}$ with microvascular dysfunction. Microcirculation 2002, 9:147-60.

449. Roberts JM and Lain KY: Recent Insights into the pathogenesis of pre-eclampsia. Placenta 2002, 23:359-72.

450. Vaughan JE and Walsh SW: Oxidative stress reproduces placental abnormalities of preeclampsia. Hypertens Preg 2002, 2 I:205-223. 
45I. Burton G] and Jauniaux E: Placental oxidative stress: from miscarriage to preeclampsia. I Soc Gynecol Investig 2004, I I:342-352.

452. Connors $\mathrm{N}$ and Merrill D: Antioxidants for prevention of preterm delivery. Clin Obs Gynecol 2004, 47:822-832.

453. Raijmakers MT, Peters WH, Steegers EA and Poston L: Amino thiols, detoxification and oxidative stress in pre-eclampsia and other disorders of pregnancy. Curr Pharm Des 2005, I I:7| I-34.

454. Redman CW and Sargent IL: Pre-eclampsia, the placenta and the maternal systemic inflammatory response-a review. Placenta 2003, 24(Suppl A):S2I-7.

455. Downing JW, Ramasubramanian R, Johnson RF, Minzter $B H$, Paschall RL, Sundell HW, Engelhardt B and Lewis R: Hypothesis: selective phosphodiesterase- 5 inhibition improves outcome in preeclampsia. Med Hypotheses 2004, 63:1057-64.

456. Myatt $L$ and Cui $X L$ : Oxidative stress in the placenta. Histochem Cell Biol 2004, I 22:369-382.

457. Touyz RM and Schiffrin EL: Reactive oxygen species in vascular biology: implications in hypertension. Histochem Cell Biol 2004, I 22:339-52.

458. Touyz RM: Reactive oxygen species, vascular oxidative stress, and redox signaling in hypertension: what is the clinical significance? Hypertension 2004, 44:248-52.

459. Tsukahara H, Ohta N, Sato S, Hiraoka M, Shukunami K, Uchiyama M, Kawakami H, Sekine $K$ and Mayumi M: Concentrations of pentosidine, an advanced glycation end-product, in umbilical cord blood. Free Radic Res 2004, 38:69l-5.

460. Bdolah $Y$, Karumanchi SA and Sachs BP: Recent advances in understanding of preeclampsia. Croat Med J 2005, 46:728-36.

46I. Biondi C, Pavan B, Lunghi L, Florini S and Vesce F: The role and modulation of the oxidative balance in pregnancy. Curr Pharmaceut Design 2005, I I:2075-2089.

462. Engin-Üstün $Y$, Üstün $Y$, Kamaci $M$ and Şekeroğlu R: Maternal serum ceruloplasmin in preectampsia. Int J Gynecol Obs 2005, 89:51-52.

463. Gupta S, Agarwal A and Sharma RK: The role of placental oxidative stress and lipid peroxidation in preeclampsia. Obstet Gynecol Surv 2005, 60:807-16

464. Noris M, Perico N and Remuzzi G: Mechanisms of disease: preeclampsia. Nature Clin Pract Nephrol 2005, I:98-I I4.

465. Redman CW and Sargent IL: Latest advances in understanding preeclampsia. Science 2005, 308: 1592-1594.

466. Roberts JM and Gammill HS: Preeclampsia: recent insights. Hypertension 2005, 46:1243-9.

467. Rodrigo R, Parra M, Bosco C, Fernandez V, Barja P, Guajardo I and Messina R: Pathophysiological basis for the prophylaxis of preeclampsia through early supplementation with antioxidant vitamins. Pharmacology \& Therapeutics 2005, I07:177-197.

468. Scholl TO, Leskiw $M$, Chen $X$, Sims $M$ and Stein TP: Oxidative stress, diet, and the etiology of preeclampsia. Am J Clin Nutr 2005, 8 I: I390-6.

469. Soleymanlou N, Jurisica I, Nevo O, letta F, Zhang X, Zamudio S, Post $M$ and Caniggia I: Molecular evidence of placental hypoxia in preeclampsia. J Clin Endocrinol Metab 2005, 90:4299-4308.

470. Spinnato JA and Livingston JC: Prevention of preeclampsia with antioxidants: Evidence from randomized trials. Clinical Obstetrics and Gynecology 2005, 48:416-429.

47I. Blackburn S: Free radicals in perinatal and neonatal care, part 2 - Oxidative stress during the perinatal and neonatal period. Journal of Perinatal \& Neonatal Nursing 2006, 20:|25-|27.

472. Borzychowski AM, Sargent IL and Redman CW: Inflammation and pre-eclampsia. Semin Fetal Neonatal Med 2006, I I:309-16.

473. Hauguel-de Mouzon S and Guerre-Millo M: The placenta cytokine network and inflammatory signals. Placenta 2006, 27:794-8.

474. Perkins AV: Endogenous anti-oxidants in pregnancy and preeclampsia. Aus New Zeal J Obs Gynaecol 2006, 46:77-83.

475. Rogers MS, Wang CC, Tam WH, Li CY, Chu KO and Chu CY. Oxidative stress in midpregnancy as a predictor of gestational hypertension and pre-eclampsia. BJOG 2006, I 13:1053-9.

476. Sankaralingam S, Arenas IA, Lalu MM and Davidge ST: Preeclampsia: current understanding of the molecular basis of vascular dysfunction. Expert Rev Mol Med 2006, 8: I-20.

477. Sargent IL, Borzychowski AM and Redman CW: Immunoregulation in normal pregnancy and pre-eclampsia: an overview. Reprod Biomed Online 2006, 13:680-6.

478. Sharma JB, Sharma A, Bahadur A, Vimala N, Satyam A and Mittal S: Oxidative stress markers and antioxidant levels in normal pregnancy and pre-eclampsia. Int J Gynecol Obs 2006, 94:23-27.
479. Webster RP, Brockman D and Myatt L: Nitration of p38 MAPK in the placenta: association of nitration with reduced catalytic activity of p38 MAPK in pre-eclampsia. Molecular Human Reproduction 2006, I 2:677-685.

480. Burdon C Mann C Cindrova-Davies T, Ferguson-Smith AC and Burton GJ: Oxidative stress and the induction of cyclooxygenase enzymes and apoptosis in the murine placenta. Placenta 2007, 28:724-33.

48I. Crocker I: Gabor Than Award Lecture 2006: pre-eclampsia and villous trophoblast turnover: perspectives and possibilities. Placenta 2007, 28(Suppl A):S4-I3.

482. Keelan JA and Mitchell MD: Placental cytokines and preeclampsia. Front Biosci 2007, I 2:2706-27

483. Milczarek R, Sokolowska E, Hallmann A and Klimek J: The NADPH- and iron-dependent lipid peroxidation in human placental microsomes. Molecular and Cellular Biochemistry 2007, 295: $105-111$.

484. Schiessl B: Inflammatory response in preeclampsia. Mol Aspects Med 2007, 28:210-9.

485. Shah TJ and Walsh SW: Activation of NF-kappaB and expression of COX-2 in association with neutrophil infiltration in systemic vascular tissue of women with preeclampsia. Am J Obstet Gynecol 2007, 196:48 el-8.

486. Walsh SW: Obesity: a risk factor for preeclampsia. Trends Endocrinol Metab 2007, I 8:365-70.

487. Zamudio S: High-altitude hypoxia and preeclampsia. Frontiers in Bioscience 2007, I 2:2967-2977.

488. Gilbert JS, Ryan MJ, LaMarca BB, Sedeek M, Murphy SR and Granger JP: Pathophysiology of hypertension during preeclampsia: linking placental ischemia with endothelial dysfunction. Am J Physiol Heart Circ Physiol 2008, 294:H54I-50.

489. Robinson NJ, Wareing M, Hudson NK, Blankley RT, Baker PN, Aplin JD and Crocker IP: Oxygen and the liberation of placental factors responsible for vascular compromise. Lab Invest 2008.

490. Entman SS, Richardson LD and Killam AP: Elevated serum ferritin in the altered ferrokinetics of toxemia of pregnancy. AmJ Obs Gynecol 1982, 144:418-422.

49I. Entman SS and Richardson LD: Clinical applications of the altered iron kinetics of toxemia of pregnancy. Am J Obs Gynecol 1983, | 46:568-574.

492. Entman SS, Richardson LD and Killam AP: Altered ferrokinetics in toxemia of pregnancy - a possible indicator of decreased red cell survival. Clin Exp Hypertens B 1983, 2: I7I-178.

493. Entman SS, Kambam JR, Bradley CA and Cousar JB: Increased levels of carboxyhemoglobin and serum iron as an indicator of increased red cell turnover in preeclampsia. Am J Obstet Gynecol 1987, I 56: I I69-73.

494. Samuels P, Main EK, Mennuti MT and Gabbe SG: The origin of increased serum iron in pregnancy-induced hypertension. Am J Obstet Gynecol 1987, I 57:72I-5.

495. Lindheimer MD and Katz Al: Preeclampsia - Patho-physiology, diagnosis, and management. Annual Review of Medicine 1989, 40:233-250.

496. Raman L, Pawashe $A B$ and Yasodhara P: Hyperferritinemia in pregnancy induced hypertension and eclampsia. J Postgrad Med 1992, 38:65-7.

497. Das SS, Dhall GI, Dhall K and Dash S: Significance of serum iron levels as a biochemical marker in pregnancy-induced hypertension. Int J Gynecol Obs 1994, 45:3-9.

498. Hubel CA Kozlov AV, Kagan VE, Evans RW, Davidge ST, McLaughlin MK and Roberts JM: Decreased transferrin and increased transferrin saturation in sera of women with preeclampsia: implications for oxidative stress. Am J Obstet Gynecol 1996, I 75:692-700.

499. Sizoo BB, Paarlberg MM, Bouman AA and Dekker GA: The role of serum iron levels in diagnostic hypertensive disorders in pregnancy. Hypertension in Pregnancy 1997, 16:425-433.

500. Vitoratos N, Salamalekis E, Dalamaga N, Kassanos D and Creatsas G: Defective antioxidant mechanisms via changes in serum ceruloplasmin and total iron binding capacity of serum in women with pre-eclampsia. Eur J Obstet Gynecol Reprod Biol 1999, 84:63-7.

50I. Lao TT, Tam KF and Chan LY: Third trimester iron status and pregnancy outcome in non-anaemic women; pregnancy unfavourably affected by maternal iron excess. Hum Reprod 2000, I 5: 1843-8.

502. Waterstone $M$, Bewley $S$ and Wolfe $C$ : Incidence and predictors of severe obstetric morbidity: case-control study. BMJ $200 \mathrm{I}$, 322: 1089-93. 
503. Mello G, Parretti E, Cioni R, Lagozio C, Mealli F and Pratesi M: Individual longitudinal patterns in biochemical and hematological markers for the early prediction of pre-eclampsia. J Matern Fetal Neonatal Med 2002, I I:93-9.

504. Casanueva E and Viteri FE: Iron and oxidative stress in pregnancy. J Nutr 2003, I33: I700S-I708S.

505. Hubel CA, Bodnar LM, Many A, Harger G, Ness RB and Roberts JM: Nonglycosylated ferritin predominates in the circulation of women with preeclampsia but not intrauterine growth restriction. Clin Chem 2004, 50:948-951.

506. Norwitz ER, Tsen LC, Park JS, Fitzpatrick PA, Dorfman DM, Saade GR, Buhimschi CS and Buhimschi IA: Discriminatory proteomic biomarker analysis identifies free hemoglobin in the cerebrospinal fluid of women with severe preeclampsia. Amer J Obs Gynecol 2005, 193:957-964.

507. Scholl TO: Iron status during pregnancy: setting the stage for mother and infant. Am J Clin Nutr 2005, 81: I $218 \mathrm{~S}-1222 \mathrm{~S}$.

508. Serdar Z, Gür E and Develioğlu O: Serum iron and copper status and oxidative stress in severe and mild preeclampsia. Cell Biochem Funct 2006, 24:209-I5.

509. Smith TG and Robbins PA: Iron, pre-eclampsia and hypoxiainducible factor. Bjog 2007, I |4:|58|-2.

510. Ziaei S, Norrozi M, Faghihzadeh S and Jafarbegloo E: A randomised placebo-controlled trial to determine the effect of iron supplementation on pregnancy outcome in pregnant women with haemoglobin $>$ or $=13.2 \mathrm{~g} / \mathrm{dl}$. BJOG 2007, I | 4:684-8.

5II. Guller S, Buhimschi CS, Ma YY, Huang ST, Yang L, Kuczynski E, Zambrano E, Lockwood CJ and Buhimschi IA: Placental expression of ceruloplasmin in pregnancies complicated by severe preeclampsia. Lab Invest 2008, 88: 1057-67.

512. Gambling L, Danzeisen R, Gair S, Lea RG, Charania Z, Solanky N, Joory KD, Srai SKS and McArdle HJ: Effect of iron deficiency on placental transfer of iron and expression of iron transport proteins in vivo and in vitro. Biochem J 200I, 356:883-889.

513. Freeman DJ, McManus F, Brown EA, Cherry L, Norrie J, Ramsay JE, Clark P, Walker ID, Sattar N and Greer IA: Short- and long-term changes in plasma inflammatory markers associated with preeclampsia. Hypertension 2004, 44:708-I4.

514. Bodnar LM, Ness RB, Harger GF and Roberts JM: Inflammation and triglycerides partially mediate the effect of prepregnancy body mass index on the risk of preeclampsia. $\mathrm{Am} \mathrm{J}$ Epidemiol 2005, I 62: I 198-206.

515. Braekke K, Holthe MR, Harsem NK, Fagerhol MK and Staff AC: Calprotectin, a marker of inflammation, is elevated in the maternal but not in the fetal circulation in preeclampsia. Am J Obstet Gynecol 2005, I 93:227-33.

5I6. Hu W, Wang $\mathrm{H}$, Wang $\mathrm{Z}$, Huang $\mathrm{H}$ and Dong $M$ : Elevated serum levels of interleukin- I5 and interleukin- I 6 in preeclampsia. J Reprod Immunol 2007, 73:166-7I.

517. Keelan JA and Mitchell MD: Cytokines, hypoxia, and preeclampsia. J Soc Gynecol Investig 2005, I 2:385-7.

518. Elovitz MA: Anti-inflammatory interventions in pregnancy: now and the future. Semin Fetal Neonatal Med 2006, II:327-32

519. Paternoster DM, Fantinato S, Stella A, Nanhorngue KN, Milani M, Plebani $M$, Nicolini $U$ and Girolami A: C-reactive protein in hypertensive disorders in pregnancy. Clin Appl Thromb Hemost 2006, I 2:330-7.

520. Dorrepaal CA, Berger HM, Benders M, vanZoerenGrobben D, VanDeBor $M$ and VanBel F: Nonprotein-bound iron in postasphyxial reperfusion injury of the newborn. Pediatrics 1996 , 98:883-889.

521. Goldenberg RL, Tamura T, DuBard M, Johnston KE, Copper RL and Neggers Y: Plasma ferritin and pregnancy outcome. Am J Obs Gynecol 1996, I 75:1356-1359.

522. Tamura T, Goldenberg RL, Johnston KE, Cliver SP and Hickey CA: Serum ferritin: A predictor of early spontaneous preterm delivery. Obs Gynecol 1996, 87:360-365.

523. Buhimschi IA, Buhimschi CS and Weiner CP: Protective effect of $\mathbf{N}$-acetylcysteine against fetal death and preterm labor induced by maternal inflammation. American Journal of Obstetrics and Gynecology 2003, I88:203-208.

524. Ciccoli L, Rossi V, Leoncini S, Signorini C, Paffetti P, Bracci R, Buonocore $\mathrm{G}$ and Comporti $\mathrm{M}$ : Iron release in erythrocytes and plasma non protein-bound iron in hypoxic and non hypoxic newborns. Free Radical Research 2003, 37:5I-58.

525. Buhimschi IA, Saade GR, Chwalisz K and Garfield RE: The nitric oxide pathway in pre-eclampsia: pathophysiological implications. Human Reproduction Update 1998, 4:25-42.
526. Milman N: Iron and pregnancy - a delicate balance. Annals of Hematology 2006, 85:559-565.

527. Milman N: Iron prophylaxis in pregnancy-general or individual and in which dose? Annals of Hematology 2006, 85:82I-828.

528. Lachili B, Hininger I, Faure H, Arnaud J, Richard MJ, Favier A and Roussel AM: Increased lipid peroxidation in pregnant women after iron and vitamin $\mathbf{C}$ supplementation. Biol Trace Elem Res 200।, 83: $103-10$

529. Devrim E, Tarhan I, Ergüder IB and Durak I: Oxidant/antioxidant status of placenta, blood, and cord blood samples from pregnant women supplemented with iron. J Soc Gynecol Investig 2006, I3:502-5.

530. Keyes LE, Armaza JF, Niermeyer S, Vargas E, Young DA and Moore LG: Intrauterine growth restriction, preeclampsia, and intrauterine mortality at high altitude in Bolivia. Pediatric Research 2003, 54:20-25.

531. Teramo KA, Hiilesmaa VK, Schwartz R, Clemons GK and Widness JA: Amniotic fluid and cord plasma erythropoietin levels in pregnancies complicated by preeclampsia, pregnancy-induced hypertension and chronic hypertension. J Perinat Med 2004, 32:240-7.

532. Liang ST, Wong VC, So WW, Ma HK, Chan V and Todd D: Homozygous alpha-thalassaemia: clinical presentation, diagnosis and management. A review of 46 cases. $\mathrm{Br} J$ Obstet Gynaecol 1985, 92:680-4.

533. Tungwiwat $W$, Fucharoen $S$, Fucharoen $G$, Ratanasiri $T$ and Sanchaisuriya K: Development and application of a real-time quantitative PCR for prenatal detection of fetal alpha(0)thalassemia from maternal plasma. Ann N Y Acad Sci 2006, 1075: 103-7.

534. Senden IP, de Groot CJ, Steegers EA, Bertina RM and Swinkels DW: Preeclampsia and the C282Y mutation in the hemochromatosis (HFE) gene. Clin Chem 2004, 50:973-4.

535. Quek L and Thein SL: Molecular therapies in beta-thalassaemia. British Journal of Haematology 2007, I36:353-365.

536. Knox KS and Baker JC: Genome-wide expression profiling of placentas in the p57Kip2 model of pre-eclampsia. Mol Hum Reprod 2007, I3:251-63.

537. Suire S, Stewart F, Beauchamp J and Kennedy MW: Uterocalin, a lipocalin provisioning the preattachment equine conceptus: fatty acid and retinol binding properties, and structural characterization. Biochem J 200I, 356:369-76.

538. Ryon J, Bendickson L and Nilsen-Hamilton M: High expression in involuting reproductive tissues of uterocalin $/ 24 \mathrm{p} 3$, a lipocalin and acute phase protein. Biochem J 2002, 367:27I-7.

539. Nilsen-Hamilton M, Liu Q, Ryon J, Bendickson L, Lepont $P$ and Chang Q: Tissue involution and the acute phase response. Ann N Y Acad Sci 2003, 995:94-108.

540. D'Anna R, Baviera G, Giordano D, Todarello G, Corrado F and Buemi M: Second trimester neutrophil gelatinase-associated lipocalin as a potential prediagnostic marker of preeclampsia. Acta Obstet Gynecol Scand 2008, I-4.

54I. Coughlan MT, Permezel M, Georgiou HM and Rice GE: Repression of oxidant-induced nuclear factor-kappaB activity mediates placental cytokine responses in gestational diabetes. J Clin Endocrinol Metab 2004, 89:3585-94.

542. Coughlan MT, Vervaart PP, Permezel M, Georgiou HM and Rice GE: Altered placental oxidative stress status in gestational diabetes mellitus. Placenta 2004, 25:78-84.

543. King JC: Maternal obesity, metabolism and pregnancy outcomes. Annu Rev Nutrition 2006, 26:27I-29I.

544. Mazar RM, Srinivas SK, Sammel MD, Andrela CM and Elovitz MA: Metabolic score as a novel approach to assessing preeclampsia risk. Am J Obstet Gynecol 2007, I97:4 I I el-5.

545. Rademacher TW, Gumaa K and Scioscia M: Preeclampsia, insulin signalling and immunological dysfunction: a fetal, maternal or placental disorder? J Reprod Immunol 2007, 76:78-84.

546. Hales CN and Ozanne SE: For Debate: Fetal and early postnatal growth restriction lead to diabetes, the metabolic syndrome and renal failure. Diabetologia 2003, 46:1013-1019.

547. McMillen IC and Robinson JS: Developmental origins of the metabolic syndrome: prediction, plasticity, and programming. Physiol Rev 2005, 85:57I-633.

548. Williams PJ, Gumaa K, Scioscia M, Redman CW and Rademacher TW: Inositol phosphoglycan P-type in preeclampsia: a novel marker? Hypertension 2007, 49:84-9.

549. West IC: Radicals and oxidative stress in diabetes. Diabet Med 2000, 17:171-80.

550. Lipinski B: Pathophysiology of oxidative stress in diabetes mellitus. J Diabetes Complications 200I, I5:203-I0. 
55I. Maddux BA, See W, Lawrence JC Jr, Goldfine AL, Goldfine ID and Evans JL: Protection against oxidative stress-induced insulin resistance in rat L6 muscle cells by mircomolar concentrations of alpha-lipoic acid. Diabetes 2001, 50:404-10.

552. Evans JL, Maddux BA and Goldfine ID: The molecular basis for oxidative stress-induced insulin resistance. Antioxid Redox Signal 2005, 7:1040-52.

553. Houstis N, Rosen ED and Lander ES: Reactive oxygen species have a causal role in multiple forms of insulin resistance. Nature 2006, 440:944-8.

554. Meigs JB, Larson MG, Fox CS, Keaney JF Jr, Vasan RS and Benjamin EJ: Association of oxidative stress, insulin resistance, and diabetes risk phenotypes: the Framingham Offspring Study. Diabetes Care 2007, 30:2529-35.

555. Shah S, Iqbal M, Karam J, Salifu M and McFarlane SI: Oxidative stress, glucose metabolism, and the prevention of type 2 diabetes: Pathophysiological insights. Antioxid Redox Signal 2007, 9:91।-929.

556. Choi SW, Benzie IF, Ma SW, Strain J J and Hannigan BM: Acute hyperglycemia and oxidative stress: direct cause and effect? Free Radic Biol Med 2008, 44:|2 I7-3|.

557. Nishikawa T, Edelstein D, Du XL, Yamagishi S, Matsumura $T$, Kaneda Y, Yorek MA, Beebe D, Oates PJ, Hammes HP, Giardino I and Brownlee $M$ : Normalizing mitochondrial superoxide production blocks three pathways of hyperglycaemic damage. Nature 2000, 404:787-90.

558. Evans JL, Goldfine ID, Maddux BA and Grodsky GM: Oxidative stress and stress-activated signaling pathways: A unifying hypothesis of type 2 diabetes. Endocrine Reviews 2002 , 23:599-622.

559. Niedowicz DM and Daleke DL: The role of oxidative stress in diabetic complications. Cell Biochem Biophys 2005, 43:289-330.

560. Kelley DE, He J, Menshikova EV and Ritov VB: Dysfunction of mitochondria in human skeletal muscle in type 2 diabetes. Diabetes 2002, 5 1:2944-50.

56I. Inoue I, Katayama S, Takahashi K, Negishi K, Miyazaki T, Sonoda M and Komoda T: Troglitazone has a scavenging effect on reactive oxygen species. Biochem Biophys Res Commun 1997, 235: I 13-6.

562. Fukui T, Noma T, Mizushige K, Aki Y, Kimura S and Abe Y: Dietary troglitazone decreases oxidative stress in early stage type II diabetic rats. Life Sci 2000, 66:2043-9.

563. Bao Y, Jia RH, Yuan J and Li J: Rosiglitazone ameliorates diabetic nephropathy by inhibiting reactive oxygen species and its downstream-signaling pathways. Pharmacology 2007, 80:57-64.

564. Jung TW, Lee JY, Shim WS, Kang ES, Kim SK, Ahn CW, Lee HC and Cha BS: Rosiglitazone protects human neuroblastoma $\mathrm{SH}$ SY5Y cells against MPP+ induced cytotoxicity via inhibition of mitochondrial dysfunction and ROS production. J Neurol Sci 2007, 253:53-60.

565. Jung Y, Song S and Choi C: Peroxisome proliferator activated receptor gamma agonists suppress TNFalpha-induced ICAM-I expression by endothelial cells in a manner potentially dependent on inhibition of reactive oxygen species. Immunol Lett 2008, I I 7:63-9.

566. Allahtavakoli M, Shabanzadeh AP, Sadr SS, Parviz M and Djahanguiri B: Rosiglitazone, a peroxisome proliferatoractivated receptor-gamma ligand, reduces infarction volume and neurological deficits in an embolic model of stroke. Clin Exp Pharmacol Physiol 2006, 33:1052-8.

567. Collino M, Aragno M, Mastrocola R, Benetti E, Gallicchio M, Dianzani C, Danni O, Thiemermann $C$ and Fantozzi R: Oxidative stress and inflammatory response evoked by transient cerebral ischemia/reperfusion: effects of the PPAR-alpha agonist WY 14643. Free Radic Biol Med 2006, 41:579-89.

568. Collino M, Aragno M, Mastrocola R, Gallicchio M, Rosa AC, Dianzani C, Danni O, Thiemermann C and Fantozzi R: Modulation of the oxidative stress and inflammatory response by PPAR gamma agonists in the hippocampus of rats exposed to cerebral ischemia/reperfusion. Eur J Pharmacol 2006, 530:70-80.

569. Allahtavakoli M, Shabanzadeh A, Roohbakhsh A and Pourshanazari A Combination therapy of rosiglitazone, a peroxisome proliferator-activated receptor-gamma ligand, and NMDA receptor antagonist (MK-80I) on experimental embolic stroke in rats. Basic Clin Pharmacol Toxicol 2007, 10 I:309-14.

570. Wessel J, Moratorio G, Rao F, Mahata M, Zhang L, Greene W, Rana BK, Kennedy BP, Khandrika S, Huang P, Lillie EO, Shih PA Smith DW, Wen G, Hamilton BA, Ziegler MG, Witztum JL, Schork NJ, Schmid-Schonbein GW and O'Connor DT: C-reactive protein, an 'intermediate phenotype' for inflammation: human twin studies reveal heritability, association with blood pressure and the metabolic syndrome, and the influence of common polymorphism at catecholaminergic/ beta-adrenergic pathway loci. J Hypertens 2007, 25:329-43.

57I. Baynes JW and Thorpe SR: Role of oxidative stress in diabetic complications: a new perspective on an old paradigm. Diabetes 1999, 48: I-9.

572. Hu FB, Meigs JB, Li TY, Rifai $N$ and Manson JE: Inflammatory markers and risk of developing type 2 diabetes in women. Diabetes 2004, 53:693-700.

573. Pickup JC: Inflammation and activated innate immunity in the pathogenesis of type 2 diabetes. Diabetes Care 2004, 27:8I3-23.

574. Kolb $\mathrm{H}$ and Mandrup-Poulsen T: An immune origin of type 2 diabetes? Diabetologia 2005, 48: I038-50.

575. Lee $\mathrm{YH}$ and Pratley RE: The evolving role of inflammation in obesity and the metabolic syndrome. Curr Diab Rep 2005, 5:70-5.

576. Tataranni PA and Ortega E: A burning question: does an adipokine-induced activation of the immune system mediate the effect of overnutrition on type $\mathbf{2}$ diabetes? Diabetes 2005, 54:917-27

577. Wellen KE and Hotamisligil GS: Inflammation, stress, and diabetes. I Clin Invest 2005, I I 5: I I II-9.

578. Alexandraki K, Piperi C, Kalofoutis C, Singh J, Alaveras A and Kalofoutis A: Inflammatory process in type 2 diabetes: The role of cytokines. Ann N Y Acad Sci 2006, 1084:89-II7.

579. Chen $\mathrm{H}$ : Cellular inflammatory responses: novel insights for obesity and insulin resistance. Pharmacol Res 2006, 53:469-77.

580. Herder C, Peltonen M, Koenig W, Kraft I, Muller-Scholze S, Martin S, Lakka T, llanne-Parikka P, Eriksson JG, Hamalainen H, KeinanenKiukaanniemi S, Valle TT, Uusitupa M, Lindstrom J, Kolb $\mathrm{H}$ and Tuomilehto J: Systemic immune mediators and lifestyle changes in the prevention of type 2 diabetes: results from the Finnish Diabetes Prevention Study. Diabetes 2006, 55:2340-6

581. Hotamisligil GS: Inflammation and metabolic disorders. Nature 2006, 444:860-7.

582. Kempf K, Rose B, Herder C, Kleophas U, Martin S and Kolb H: Inflammation in metabolic syndrome and type 2 diabetes: Impact of dietary glucose. Ann N Y Acad Sci 2006, 1084:30-48.

583. Neels JG and Olefsky JM: Inflamed fat: what starts the fire? J Clin Invest 2006, I 1 6:33-5.

584. Shoelson SE, Lee J and Goldfine AB: Inflammation and insulin resistance. I Clin Invest 2006, I I 6: |793-80 I.

585. Weisberg SP, Hunter D, Huber R, Lemieux J, Slaymaker S, Vaddi K, Charo I, Leibel RL and Ferrante AW Jr: CCR2 modulates inflammatory and metabolic effects of high-fat feeding. J Clin Invest 2006, I | 6: I I 5-24.

586. Zozulinska D and Wierusz-Wysocka B: Type 2 diabetes mellitus as inflammatory disease. Diabetes Research and Clinical Practice 2006, 74:SI2-S16.

587. Pradhan A: Obesity, metabolic syndrome, and type 2 diabetes: inflammatory basis of glucose metabolic disorders. Nutr Rev 2007, 65:SI52-6.

588. Shoelson SE, Herrero $L$ and Naaz A: Obesity, inflammation, and insulin resistance. Gastroenterology 2007, I32:2 I69-2180.

589. Thorand B, Baumert J, Kolb H, Meisinger C, Chambless L, Koenig W and Herder C: Sex differences in the prediction of type 2 diabetes by inflammatory markers: results from the MONICA/KORA Augsburg case-cohort study, 1984-2002. Diabetes Care 2007, 30:854-60.

590. Wei Y, Chen K, Whaley-Connell AT, Stump CS, Ibdah JA and Sowers JR: Skeletal muscle insulin resistance: role of inflammatory cytokines and reactive oxygen species. Am Physiol Regul Integr Comp Physiol 2008, 294:R673-80.

591. Lao TT, Chan PL and Tam KF: Gestational diabetes mellitus in the last trimester - a feature of maternal iron excess? Diabet Med 200I, I 8:218-23.

592. Lao TT, Tse KY, Chan LY, Tam KF and Ho LF: NBsAg carrier status and the association between gestational diabetes with increased serum ferritin concentration in Chinese women. Diabetes Care 2003, 26:3011-3016.

593. Chen X, Scholl TO and Stein TP: Association of elevated serum ferritin levels and the risk of gestational diabetes mellitus in pregnant women: The Camden study. Diabetes Care 2006, 29: $1077-82$.

594. Oberley LW: Free radicals and diabetes. Free Radic Biol Med 1988, 5:113-24.

595. Wolff SP: Diabetes mellitus and free radicals. Free radicals, transition metals and oxidative stress in the aetiology of 
diabetes mellitus and complications. Br Med Bull 1993, 49:642-52.

596. Tuomainen TP, Nyyssonen K, Salonen R, Tervahauta A, Korpela H, Lakka T, Kaplan GA and Salonen IT: Body iron stores are associated with serum insulin and blood glucose concentrations. Population study in I,0 I 3 eastern Finnish men. Diabetes Care 1997, 20:426-8.

597. Salonen JT, Tuomainen TP, Nyyssonen K, Lakka HM and Punnonen K: Relation between iron stores and non-insulin dependent diabetes in men: case-control study. BMJ I998, 3 1 7:727.

598. Fernández-Real JM, López-Bermejo A and Ricart W: Cross-talk between iron metabolism and diabetes. Diabetes 2002, 5 I:2348-54

599. Fernández-Real JM and Ricart W: Insulin resistance and chronic cardiovascular inflammatory syndrome. Endocr Rev 2003, 24:278-301.

600. Wilson JG, Lindquist JH, Grambow SC, Crook ED and Maher JF: Potential role of increased iron stores in diabetes. Am J Med Sci 2003, 325:332-9.

60I. Lee DH, Folsom AR and Jacobs DR Jr: Dietary iron intake and Type 2 diabetes incidence in postmenopausal women: the lowa Women's Health Study. Diabetologia 2004, 47: I85-94.

602. Kazi TG, Afridi HI, Kazi N, Jamali MK, Arain MB, Jalbani N and Kandhro GA: Copper, chromium, manganese, iron, nickel, and zinc levels in biological samples of diabetes mellitus patients. Biol Trace Elem Res 2008, I 22:1-18.

603. Rajpathak SN, Crandall JP, Wylie-Rosett J, Kabat GC, Rohan TE and Hu FB: The role of iron in type 2 diabetes in humans. Biochim Biophys Acta 2008 in press.

604. Adams PC, Kertesz AE and Valberg LS: Clinical presentation of hemochromatosis: a changing scene. Am J Med I991, 90:445-9.

605. Kaye TB, Guay AT and Simonson DC: Non-insulin-dependent diabetes mellitus and elevated serum ferritin level. J Diabetes Complic 1993, 7:246-249.

606. Fernández-Real JM, Ricart-Engel W, Arroyo E, Balanca R, Casamitjana-Abella R, Cabrero D, Fernandez-Castaner $M$ and Soler J: Serum ferritin as a component of the insulin resistance syndrome. Diabetes Care 1998, 21:62-68.

607. Ford ES and Cogswell ME: Diabetes and serum ferritin concentration among U.S. adults. Diabetes Care 1999, 22: 1978-83.

608. Jiang R, Manson JE, Meigs JB, Ma J, Rifai $\mathrm{N}$ and Hu FB: Body iron stores in relation to risk of type 2 diabetes in apparently healthy women. JAMA 2004, 291:71I-7.

609. Mojiminiyi OA, Marouf $R$ and Abdella NA: Body iron stores in relation to the metabolic syndrome, glycemic control and complications in female patients with type 2 diabetes. Nutr Metab Cardiovasc Dis 2008, 18:559-66.

610. Jehn ML, Guallar E, Clark JM, Couper D, Duncan BB, Ballantyne CM, Hoogeveen RC, Harris ZL and Pankow JS: A prospective study of plasma ferritin level and incident diabetes: the Atherosclerosis Risk in Communities (ARIC) Study. Am J Epidemiol 2007, 1 65:1047-54.

6II. Fernández-Real JM, Moreno JM, Chico B, Lopez-Bermejo A and Ricart W: Circulating visfatin is associated with parameters of iron metabolism in subjects with altered glucose tolerance. Diabetes Care 2007, 30:616-21.

612. Wang Y, Lam KSL, Kraegen EW, Sweeney G, Zhang JL, Tso AWK, Chow WS, Wat NMS, Xu JY, Hoo RLC and Xu AM: Lipocalin-2 is an inflammatory marker closely associated with obesity, insulin resistance, and hyperglycemia in humans. Clinical Chemistry 2007, 53:34-4I.

613. van Dam RM and Hu FB: Lipocalins and insulin resistance: Etiological role of retinol-binding protein 4 and lipocalin-2? Clinical Chemistry 2007, 53:5-7.

614. Hua NW, Stoohs RA and Facchini FS: Low iron status and enhanced insulin sensitivity in lacto-ovo vegetarians. $\mathrm{Br}$ 」 Nutr 200I, 86:5I5-9.

615. Liang Q, Carlson EC, Donthi RV, Kralik PM, Shen X and Epstein PN: Overexpression of metallothionein reduces diabetic cardiomyopathy. Diabetes 2002, 5 I:I74-81.

616. Ye G, Metreveli NS, Ren J and Epstein PN: Metallothionein prevents diabetes-induced deficits in cardiomyocytes by inhibiting reactive oxygen species production. Diabetes 2003 , 52:777-83.

617. Cai L, Wang J, Li Y, Sun X, Wang L, Zhou Z and Kang YJ: Inhibition of superoxide generation and associated nitrosative damage is involved in metallothionein prevention of diabetic cardiomyopathy. Diabetes 2005, 54:1829-37.
618. Islam MS and Loots du T: Diabetes, metallothionein, and zinc interactions: a review. Biofactors 2007, 29:203-12.

619. Li X, Cai L and Feng W: Diabetes and metallothionein. Mini Rev Med Chem 2007, 7:76I-8.

620. Zheng Y, Li XK, Wang Y and Cai L: The role of zinc, copper and iron in the pathogenesis of diabetes and diabetic complications: therapeutic effects by chelators. Hemoglobin 2008 , 32: I 35-45.

621. Lee DH, Liu DY, Jacobs DR Jr, Shin HR, Song K, Lee IK, Kim B and Hider RC: Common presence of non-transferrin-bound iron among patients with type 2 diabetes. Diabetes Care 2006, 29:1090-5.

622. Chittum HS and Champney WS: Erythromycin inhibits the assembly of the large ribosomal subunit in growing Escherichia coli cells. Curr Microbiol 1995, 30:273-9.

623. Facchini FS, Hua NW and Stoohs RA: Effect of iron depletion in carbohydrate-intolerant patients with clinical evidence of nonalcoholic fatty liver disease. Gastroenterology 2002, I 22:93 I-9.

624. Machado $M$ and Cortez-Pinto $H$ : Nash, insulin resistance and iron. Liver International 2006, 26: I|59-1|62.

625. Allerson $C R$, Cazzola $M$ and Rouault TA: Clinical severity and thermodynamic effects of iron-responsive element mutations in hereditary hyperferritinemia-cataract syndrome. Journal of Biological Chemistry 1999, 274:26439-26447.

626. Beaumont C, Leneuve P, Devaux I, Scoazec JY, Berthier M, Loiseau MN, Grandchamp B and Bonneau D: Mutation in the Iron-Responsive Element of the L-Ferritin Messenger-Rna in a Family with Dominant Hyperferritinemia and Cataract. Nature Genetics 1995, I I:444-446.

627. Dandona P, Aljada A, Chaudhuri A, Mohanty P and Garg R. Metabolic syndrome - A comprehensive perspective based on interactions between obesity, diabetes, and inflammation. Circulation 2005, I I I:|448-1454.

628. Eckel RH, Grundy SM and Zimmet PZ: The metabolic syndrome. Lancet 2005, 365:1415-28.

629. Roberts CK and Barnard RJ: Effects of exercise and diet on chronic disease. I Appl Physiol 2005, 98:3-30.

630. Nicolson GL: Metabolic syndrome and mitochondrial function: Molecular replacement and antioxidant supplements to prevent membrane peroxidation and restore mitochondrial function. Journal of Cellular Biochemistry 2007, I 00: I352-1369.

631. Yudkin JS: Insulin resistance and the metabolic syndrome-or the pitfalls of epidemiology. Diabetologia 2007, 50: I576-86.

632. Jehn M, Clark JM and Guallar E: Serum ferritin and risk of the metabolic syndrome in U.S. adults. Diabetes Care 2004, 27:2422-8.

633. Wrede CE, Buettner R, Bollheimer LC, Scholmerich J, Palitzsch KD and Hellerbrand $C$ : Association between serum ferritin and the insulin resistance syndrome in a representative population. Eur J Endocrinol 2006, I 54:333-340.

634. Bozzini C, Girelli D, Olivieri O, Martinelli N, Bassi A, De Matteis G, Tenuti I, Lotto V, Friso S, Pizzolo F and Corrocher R: Prevalence of body iron excess in the metabolic syndrome. Diabetes Care 2005, 28:206I-3.

635. Choi KM, Lee KW, Kim HY, Seo JA, Kim SG, Kim NH, Choi DS and Baik SH: Association among serum ferritin, alanine aminotransferase levels, and metabolic syndrome in Korean postmenopausal women. Metabolism 2005, 54:1510-4.

636. Kurella M, Lo JC and Chertow GM: Metabolic syndrome and the risk for chronic kidney disease among nondiabetic adults. J Am Soc Nephrol 2005, I 6:2 I34-2|40.

637. den Boer M, Voshol PJ, Kuipers F, Havekes LM and Romijn JA: Hepatic steatosis: a mediator of the metabolic syndrome. Lessons from animal models. Arterioscler Thromb Vasc Biol 2004, 24:644-9.

638. Mittra S, Bansal VS and Bhatnagar PK: From a glucocentric to a lipocentric approach towards metabolic syndrome. Drug Discov Today 2008, I3:21 I-8.

639. Roberts CK, Barnard RJ, Sindhu RK, Jurczak M, Ehdaie A and Vaziri ND: Oxidative stress and dysregulation of NAD(P)H oxidase and antioxidant enzymes in diet-induced metabolic syndrome. Metabolism 2006, 55:928-34.

640. Booth FW, Gordon SE, Carlson Cl and Hamilton MT: Waging war on modern chronic diseases: primary prevention through exercise biology. J Appl Physiol 2000, 88:774-87.

64I. Wild S, Roglic G, Green A, Sicree R and King H: Global prevalence of diabetes: estimates for the year 2000 and projections for 2030. Diabetes Care 2004, 27:1047-53 
642. Kahn SE, Hull RL and Utzschneider KM: Mechanisms linking obesity to insulin resistance and type 2 diabetes. Nature 2006, 444:840-6.

643. Walker CG, Zariwala MG, Holness MJ and Sugden MC: Diet, obesity and diabetes: a current update. Clin Sci (Lond) 2007, I | 2:93-III.

644. Lecube A, Hernandez C, Pelegri D and Simo R: Factors accounting for high ferritin levels in obesity. Int J Obes (Lond) 2008.

645. Fricker J, Lemoel G and Apfelbaum M: Obesity and iron status in menstruating women. Am J Clin Nutrition 1990, 52:863-866.

646. Farahani P, Chiu S, Bowlus CL, Boffelli D, Lee E, Fisler JS, Krauss RM and Warden $\mathrm{CH}$ : Obesity in BSB mice is correlated with expression of genes for iron homeostasis and leptin. Obesity Research 2004, I2:19|-204.

647. Das UN: Is obesity an inflammatory condition? Nutrition 200I, I 7:953-66.

648. Hall JE, Crook ED, Jones DW, Wofford MR and Dubbert PM: Mechanisms of obesity-associated cardiovascular and renal disease. Am J Med Sci 2002, 324: I27-37.

649. Trayhurn $P$ and Wood IS: Adipokines: inflammation and the pleiotropic role of white adipose tissue. Br J Nutr 2004, 92:347-55.

650. Berg $\mathrm{AH}$ and Scherer PE: Adipose tissue, inflammation, and cardiovascular disease. Circ Res 2005, 96:939-49.

65I. Hukshorn CJ, Lindeman JH, Toet KH, Saris WH, Eilers PH, Westerterp-Plantenga MS and Kooistra $\mathrm{T}$ : Leptin and the proinflammatory state associated with human obesity. J Clin Endocrinol Metab 2004, 89:1773-8.

652. Toni R, Malaguti A, Castorina S, Roti E and Lechan RM: New paradigms in neuroendocrinology: relationships between obesity, systemic inflammation and the neuroendocrine system. J Endocrinol Invest 2004, 27: 182-6.

653. Koh KK, Han SH and Quon MJ: Inflammatory markers and the metabolic syndrome: insights from therapeutic interventions. J Am Coll Cardiol 2005, 46:1978-85.

654. Bastard IP, Maachi M, Lagathu C, Kim MJ, Caron M, Vidal H, Capeau J and Feve $B$ : Recent advances in the relationship between obesity, inflammation, and insulin resistance. Eur Cytokine Netw 2006, I7:4-12.

655. Kapiotis S, Holzer G, Schaller G, Haumer $M$, Widhalm $H$, Weghuber D, Jilma B, Roggla G, Wolzt $M$, Widhalm $K$ and Wagner OF: A proinflammatory state is detectable in obese children and is accompanied by functional and morphological vascular changes. Arterioscler Thromb Vasc Biol 2006, 26:254I-6.

656. Tilg $\mathrm{H}$ and Moschen AR: Adipocytokines: mediators linking adipose tissue, inflammation and immunity. Nat Rev Immunol 2006, 6:772-83.

657. Vincent HK and Taylor AG: Biomarkers and potential mechanisms of obesity-induced oxidant stress in humans. Int J Obes (Lond) 2006, 30:400-18.

658. Wang B and Trayhurn P: Acute and prolonged effects of TNF. alpha on the expression and secretion of inflammationrelated adipokines by human adipocytes differentiated in culture. Pflugers Arch 2006, 452:418-27.

659. Chandalia $M$ and Abate $N$ : Metabolic complications of obesity: inflated or inflamed? J Diabetes Complications 2007, 2 I: |28-36.

660. Lumeng CN, Bodzin JL and Saltiel AR: Obesity induces a phenotypic switch in adipose tissue macrophage polarization. J Clin Invest 2007, I I 7: I75-84.

661. Chen Y, Zhu J, Lum PY, Yang X, Pinto S, Macneil DJ, Zhang C Lamb J, Edwards S, Sieberts SK, Leonardson A, Castellini LW, Wang S, Champy MF, Zhang B, Emilsson V, Doss S, Ghazalpour A, Horvath S, Drake TA, Lusis AJ and Schadt EE: Variations in DNA elucidate molecular networks that cause disease. Nature 2008, 452:429-435.

662. Chung J, Nguyen AK, Henstridge DC, Holmes AG, Chan MH, Mesa JL, Lancaster GI, Southgate RJ, Bruce CR, Duffy SJ, Horvath I, Mestril R, Watt MJ, Hooper PL, Kingwell BA, Vigh L, Hevener A and Febbraio MA: HSP72 protects against obesity-induced insulin resistance. Proc Natl Acad Sci USA 2008, I 05: I 739-44.

663. Emilsson V, Thorleifsson G, Zhang B, Leonardson AS, Zink F, Zhu J, Carlson S, Helgason A, Walters GB, Gunnarsdottir S, Mouy M, Steinthorsdottir V, Eiriksdottir GH, Bjornsdottir G, Reynisdottir I, Gudbjartsson D, Helgadottir A, Jonasdottir A, Jonasdottir A, Styrkarsdottir U, Gretarsdottir S, Magnusson KP, Stefansson $H$ Fossdal R, Kristjansson K, Gislason HG. Stefansson'T, Leifsson BG, Thorsteinsdottir U, Lamb JR, Gulcher JR, Reitman ML, Kong A,
Schadt EE and Stefansson K: Genetics of gene expression and its effect on disease. Nature 2008.

664. Wärnberg J and Marcos A: Low-grade inflammation and the metabolic syndrome in children and adolescents. Curr Opin Lipidol 2008, 19: I I-5.

665. Rajala MW and Scherer PE: Minireview: The adipocyte-at the crossroads of energy homeostasis, inflammation, and atherosclerosis. Endocrinology 2003, 144:3765-73.

666. Vendrell J, Broch M, Vilarrasa N, Molina A, Gomez JM, Gutierrez C, Simon I, Soler J and Richart C: Resistin, adiponectin, ghrelin, leptin, and proinflammatory cytokines: relationships in obesity. Obes Res 2004, | 2:962-7|

667. Arner P: Insulin resistance in type $\mathbf{2}$ diabetes - role of the adipokines. Curr Mol Med 2005, 5:333-9.

668. Hutley $L$ and Prins JB: Fat as an endocrine organ: relationship to the metabolic syndrome. Am J Med Sci 2005, 330:280-9.

669. Kougias $\mathrm{P}$, Chai H, Lin PH, Yao Q, Lumsden AB and Chen C: Effects of adipocyte-derived cytokines on endothelial functions: implication of vascular disease. J Surg Res 2005, I 26: I 2 I-9.

670. Lau DC, Dhillon B, Yan H, Szmitko PE and Verma S: Adipokines: molecular links between obesity and atheroslcerosis. Am Physiol Heart Circ Physiol 2005, 288:H203 I-4I.

67I. Rosen ED and Spiegelman BM: Adipocytes as regulators of energy balance and glucose homeostasis. Nature 2006, 444:847-53.

672. Kim F, Pham M, Luttrell I, Bannerman DD, Tupper J, Thaler J, Hawn TR, Raines EW and Schwartz MW: Toll-like receptor-4 mediates vascular inflammation and insulin resistance in diet-induced obesity. Circ Res 2007, 100:1589-96.

673. Shah PK: Innate immune pathway links obesity to insulin resistance. Circ Res 2007, 100:1531-3.

674. Furukawa S, Fujita T, Shimabukuro M, Iwaki M, Yamada $Y$, Nakajima $Y$, Nakayama $O$, Makishima $M$, Matsuda $M$ and Shimomura I: Increased oxidative stress in obesity and its impact on metabolic syndrome. J Clin Invest 2004, I | 4: I 752-6 I.

675. Keaney JF, Larson MG Jr, Vasan RS, Wilson PW, Lipinska I, Corey D, Massaro JM, Sutherland P, Vita JA and Benjamin EJ: Obesity and systemic oxidative stress: clinical correlates of oxidative stress in the Framingham Study. Arterioscler Thromb Vasc Biol 2003, 23:434-9.

676. Higdon JV and Frei B: Obesity and oxidative stress: a direct link to CVD? Arterioscler Thromb Vasc Biol 2003, 23:365-7.

677. Whitworth JA: 2003 World Health Organization (WHO)/ International Society of Hypertension (ISH) statement on management of hypertension. J Hypertens 2003, 21:1983-92.

678. Tuder RM and Voelkel NF: Pulmonary hypertension and inflammation. J Lab Clin Med 1998, I32:16-24.

679. Intengan HD and Schiffrin EL: Vascular remodeling in hypertension: roles of apoptosis, inflammation, and fibrosis. Hypertension 200I, 38:58I-7.

680. Grundy SM: Inflammation, hypertension, and the metabolic syndrome. JAMA 2003, 290:3000-2.

681. Virdis A and Schiffrin EL: Vascular inflammation: a role in vascular disease in hypertension? Curr Opin Nephrol Hypertens 2003, 12: $181-7$

682. Boos CJ and Lip GY: Elevated high-sensitive C-reactive protein, large arterial stiffness and atherosclerosis: a relationship between inflammation and hypertension? J Hum Hypertens 2005, I 9:5 II-3.

683. Varughese GI and Lip GY: Hypertension in patients with type-II diabetes: relation to urinary albumin excretion, endothelial function and inflammation. J Hum Hypertens 2005, 19:42 I-4.

684. Li JJ: Inflammation in hypertension: primary evidence. Chin Med I (Engl) 2006, I1 9:1215-21.

685. Savoia $C$ and Schiffrin EL: Inflammation in hypertension. Curr Opin Nephrol Hypertens 2006, I 5: I52-8.

686. Savoia C and Schiffrin EL: Vascular inflammation in hypertension and diabetes: molecular mechanisms and therapeutic interventions. Clin Sci (Lond) 2007, I I 2:375-84.

687. Kannel WB, Brand N, Skinner J Jr, Dawber TR and McNamara PM: The relation of adiposity to blood pressure and development of hypertension. The Framingham study. Ann Intern Med 1967, 67:48-59.

688. Mark AL, Correia M, Morgan DA, Shaffer RA and Haynes WG: State-of-the-art-lecture: Obesity-induced hypertension: new concepts from the emerging biology of obesity. Hypertension 1999, 33:537-41.

689. Aizawa-Abe M, Ogawa Y, Masuzaki H, Ebihara K, Satoh N, Iwai H, Matsuoka N, Hayashi T, Hosoda K, Inoue G, Yoshimasa $Y$ and 
Nakao K: Pathophysiological role of leptin in obesity-related hypertension. J Clin Invest 2000, 105: I 243-52.

690. Hall JE: The kidney, hypertension, and obesity. Hypertension 2003, 41:625-33.

69l. Yanai H, Tomono Y, Ito K, Furutani N, Yoshida H and Tada N: The underlying mechanisms for development of hypertension in the metabolic syndrome. Nutr J 2008, 7:10.

692. Vaziri ND and Rodriguez-Iturbe B: Mechanisms of disease: oxidative stress and inflammation in the pathogenesis of hypertension. Nat Clin Pract Nephrol 2006, 2:582-93.

693. Yakobson MG, Antonov AR, Golovatyuk AV, Efremov AV, Markel AL and Yakobson GS: Iron content and parameters of blood antioxidant activity in rats with hereditary arterial hypertension during experimental myocardial infarction. Bull Exp Biol Med 200I, I32:104I-4.

694. Peterson JR, Sharma RV and Davisson RL: Reactive oxygen species in the neuropathogenesis of hypertension. Curr Hypertens Rep 2006, 8:232-4I.

695. Sullivan JL: Iron and the sex difference in heart-disease risk. Lancet I98I, I:1293-1294.

696. Sullivan JL: Misconceptions in the debate on the iron hypothesis. Journal of Nutritional Biochemistry 200 I, I 2:33-37.

697. Sullivan JL: Stored iron and vascular reactivity. Arteriosclerosis Thrombosis and Vascular Biology 2005, 25:1532-1535.

698. Sullivan JL: The big idea: the coxib crisis iron, aspirin and heart disease risk revisited. J R Soc Med 2007, 100:346-9.

699. Meyers DG: The iron hypothesis: does iron play a role in atherosclerosis? Transfusion 2000, 40:1023-1029.

700. Yuan XM and Li W: The iron hypothesis of atherosclerosis and its clinical impact. Ann Med 2003, 35:578-91.

70I. Zheng $H$, Dimayuga $C$, Hudaihed A and Katz SD: Effect of dexrazoxane on homocysteine-induced endothelial dysfunction in normal subjects. Arterioscler Thromb Vasc Biol 2002, 22: EI5-8.

702. Zheng $\mathrm{H}$, Huang $\mathrm{X}$, Zhang $\mathrm{Q}$ and Katz SD: Iron sucrose augments homocysteine-induced endothelial dysfunction in normal subjects. Kidney Int 2006, 69:679-84.

703. Sullivan JL: Is homocysteine an iron-dependent cardiovascular risk factor? Kidney Int 2006, 69:642-4.

704. Clarke R: Homocysteine-lowering trials for prevention of heart disease and stroke. Semin Vasc Med 2005, 5:2I 5-22.

705. Tuomainen TP, Punnonen K, Nyyssonen K and Salonen JT: Association between body iron stores and the risk of acute myocardial infarction in men. Circulation 1998, 97:|46|-6.

706. Sarnak MJ, Levey AS, Schoolwerth AC, Coresh J, Culleton B, Hamm LL, McCullough PA, Kasiske BL, Kelepouris E, Klag MJ, Parfrey P, Pfeffer M, Raij L, Spinosa DJ and Wilson PW: Kidney disease as a risk factor for development of cardiovascular disease: a statement from the American Heart Association Councils on Kidney in Cardiovascular Disease, High Blood Pressure Research, Clinical Cardiology, and Epidemiology and Prevention. Circulation 2003, 108:2154-69.

707. Oberg BP, McMenamin E, Lucas FL, McMonagle E, Morrow J, Ikizler TA and Himmelfarb J: Increased prevalence of oxidant stress and inflammation in patients with moderate to severe chronic kidney disease. Kidney Int 2004, 65:1009-16.

708. Vaziri ND: Roles of oxidative stress and antioxidant therapy in chronic kidney disease and hypertension. Curr Opin Nephrol Hypertens 2004, 13:93-9.

709. Abramson JL, Jurkovitz CT, Vaccarino V, Weintraub WS and McClellan W: Chronic kidney disease, anemia, and incident stroke in a middle-aged, community-based population: the ARIC Study. Kidney Int 2003, 64:610-5.

7I0. Smith JK, Carden DL, Grisham MB, Granger DN and Korthuis RJ: Role of iron in postischemic microvascular injury. Am J Physiol 1989, 256:HI472-7.

7II. Kukreja RC and Hess ML: The oxygen free radical system: from equations through membrane protein interactions to cardiovascular injury and protection. Cardiovasc Res 1992, 26:64I-655

712. Lesnefsky $E$ and $Y e$ J: Exogenous intracellular, but not extracellular, iron augments myocardial reperfusion injury. Amer J Physiol 1994, 266: $\mathrm{H} 384-\mathrm{H} 392$.

713. Berenshtein E, Mayer B, Goldberg C, Kitrossky N and Chevion M: Patterns of mobilization of copper and iron following myocardial ischemia: Possible predictive criteria for tissue injury. I Mol Cell Cardiol 1997, 29:3025-3034.

714. Horwitz LD, Sherman NA, Kong YN, Pike AW, Gobin J, Fennessey PV and Horwitz MA: Lipophilic siderophores Myco- bacterium tuberculosis prevent cardiac reperfusion injury. Proc Natl Acad Sci 1998, 95:5263-5268.

715. Horwitz LD and Rosenthal EA: Iron-mediated cardiovascular injury. Vasc Med 1999, 4:93-9.

7I6. Pucheu S, Coudray C, Tresallet N, Favier A and Deleiris J: Effect of iron overload in the isolated ischemic and reperfused rat heart. Cardiovascular Drugs and Therapy 1993, 7:70I-7II.

717. Amersi F, Dulkanchainun T, Nelson SK, Farmer DG, Kato H, Zaky I Melinek J, Shaw GD, Kupiec-Weglinski JW, Horwitz LD, Horwitz MA and Busuttil RW: Novel iron chelator in combination with a P-selectin antagonist prevents ischemia/reperfusion injury in a rat liver model. Transplantation 200I, 7I:112-118.

718. Mamtani $M$ and Kulkarni $\mathrm{H}$ : Influence of iron chelators on myocardial iron and cardiac function in transfusion-dependent thalassaemia: a systematic review and meta-analysis. $\mathrm{Br}$ J Haematol 2008, 141:882-90.

719. Ravati A, Ahlemeyer B, Becker A, Klumpp S and Krieglstein J: Preconditioning-induced neuroprotection is mediated by reactive oxygen species and activation of the transcription factor nuclear factor-kappa B. Journal of Neurochemistry 200I, 78:909-919.

720. Berenshtein E, Vaisman B, Goldberg-Langerman C, Kitrossky N, Konijn AM and Chevion M: Roles of ferritin and iron in ischemic preconditioning of the heart. Mol Cell Biochem 2002, 234:283-292

72I. Calvillo L, Latini R, Kajstura J, Leri A, Anversa P, Ghezzi P, Salio M, Cerami $A$ and Brines $M$ : Recombinant human erythropoietin protects the myocardium from ischemia-reperfusion injury and promotes beneficial remodeling. ProcNatl Acad Sci 2003 100:4802-4806.

722. Parsa CJ, Matsumoto A, Kim J, Riel RU, Pascal LS, Walton GB, Thompson RB, Petrofski JA, Annex BH, Stamler JS and Koch WJ: A novel protective effect of erythropoietin in the infarcted heart. I Clin Invest 2003, I I 2:999-1007.

723. Engberding N, Spiekermann S, Schaefer A, Heineke A, Wiencke A, Muller M, Fuchs M, Hilfiker-Kleiner D, Hornig B, Drexler $H$ and Landmesser U: Allopurinol attenuates left ventricular remodeling and dysfunction after experimental myocardial infarction: a new action for an old drug? Circulation 2004, II 0:2175-9.

724. Reyes AJ: Cardiovascular drugs and serum uric acid. Cardiovasc Drugs Ther 2003, I7:397-4I4.

725. Dunn WB, Broadhurst DI, Sasalu D, Buch M, McDowell G, Spasic I, Ellis DI, Brooks N, Kell DB and Neyses L. Serum metabolomics reveals many novel metabolic markers of heart failure, including pseudouridine and 2-oxoglutarate. Metabolomics 2007, 3:413-426.

726. Hokamaki J, Kawano H, Yoshimura M, Soejima H, Miyamoto S, Kajiwara I, Kojima S, Sakamoto T, Sugiyama S, Hirai N, Shimomura $H$, Nagayoshi Y, Tsujita K, Shioji I, Sasaki S and Ogawa H: Urinary biopyrrins levels are elevated in relation to severity of heart failure. J Am Coll Cardiol 2004, 43: I880-5.

727. Mozaffarian D, Nye R and Levy WC: Anemia predicts mortality in severe heart failure: the prospective randomized amlodipine survival evaluation (PRAISE). J Am Coll Cardiol 2003, $41: 1933-9$

728. Felker GM, Adams KF Jr, Gattis WA and O'Connor CM: Anemia as a risk factor and therapeutic target in heart failure. J Am Coll Cardiol 2004, 44:959-66.

729. Bolger AP, Bartlett FR, Penston HS, O'Leary J, Pollock N, Kaprielian R and Chapman CM: Intravenous iron alone for the treatment of anemia in patients with chronic heart failure. $J$ Am Coll Cardiol 2006, 48: I225-7.

730. Weiss G and Goodnough LT: Anemia of chronic disease. N Engl 」 Med 2005, 352:101।-23.

73I. Kunsch $C$ and Medford RM: Oxidative stress as a regulator of gene expression in the vasculature. Circ Res 1999, 85:753-66.

732. Ross R: Atherosclerosis: an inflammatory disease. N Engl J Med 1999, 340: II5-26.

733. Blake G] and Ridker PM: Novel clinical markers of vascular wall inflammation. Circ Res 200I, 89:763-7I.

734. Hansson GK: Immune mechanisms in atherosclerosis. Arterioscler Thromb Vasc Biol 200 I, 21: | 876-90

735. Forrester JS: Prevention of plaque rupture: a new paradigm of therapy. Ann Intern Med 2002, 137:823-33.

736. Himmelfarb J, Stenvinkel P, Ikizler TA and Hakim RM: The elephant in uremia: oxidant stress as a unifying concept of cardiovascular disease in uremia. Kidney Int 2002, 62:1524-38. 
737. Libby P: Inflammation in atherosclerosis. Nature 2002, 420:868-74.

738. Libby $P$, Ridker $P M$ and Maseri A: Inflammation and atherosclerosis. Circulation 2002, 105: I |35-43.

739. Young JL, Libby $P$ and Schonbeck U: Cytokines in the pathogenesis of atherosclerosis. Thromb Haemost 2002, 88:554-67.

740. Binder CJ, Chang MK, Shaw PX, Miller YI, Hartvigsen K, Dewan A and Witztum JL: Innate and acquired immunity in atherogenesis. Nat Med 2002, 8:1218-26.

74I. Altman R: Risk factors in coronary atherosclerosis atheroinflammation: the meeting point. Thromb / 2003, I:4.

742. Dwyer JH, Allayee H, Dwyer KM, Fan J, Wu H, Mar R, Lusis Al and Mehrabian M: Arachidonate 5-lipoxygenase promoter genotype, dietary arachidonic acid, and atherosclerosis. $N$ Engl J Med 2004, 350:29-37.

743. Forrester JS: Common ancestors: chronic progressive diseases have the same pathogenesis. Clin Cardiol 2004, 27: I86-90.

744. Paoletti R, Gotto AM Jr and Hajjar DP: Inflammation in atherosclerosis and implications for therapy. Circulation 2004, I09:III20-6.

745. Ridker PM, Brown NJ, Vaughan DE, Harrison DG and Mehta JL: Established and emerging plasma biomarkers in the prediction of first atherothrombotic events. Circulation 2004, 109 (Suppl I):6-19.

746. van Oostrom AJ, van Wijk J and Cabezas MC: Lipaemia, inflammation and atherosclerosis: novel opportunities in the understanding and treatment of atherosclerosis. Drugs 2004, 64(Suppl 2): I9-4I.

747. Willerson JT and Ridker PM: Inflammation as a cardiovascular risk factor. Circulation 2004, I09:||2-10.

748. Hansson GK: Inflammation, atherosclerosis, and coronary artery disease. N Engl J Med 2005, 352: I685-95.

749. Madamanchi NR, Hakim ZS and Runge MS. Oxidative stress in atherogenesis and arterial thrombosis: the disconnect between cellular studies and clinical outcomes. J Thromb Haemost 2005, 3:254-67.

750. Madamanchi NR, Vendrov A and Runge MS: Oxidative stress and vascular disease. Arterioscler Thromb Vasc Biol 2005, 25:29-38.

751. Mullenix PS, Andersen CA and Starnes BW: Atherosclerosis as inflammation. Ann Vasc Surg 2005, I 9: | 30-8.

752. Nigro J, Osman N, Dart AM and Little PJ: Insulin resistance and atherosclerosis. Endocr Rev 2006, 27:242-59.

753. Taqueti VR, Mitchell RN and Lichtman AH: Protecting the pump: controlling myocardial inflammatory responses. Annu Rev Physiol 2006, 68:67-95.

754. Tedgui A and Mallat Z: Cytokines in atherosclerosis: pathogenic and regulatory pathways. Physiol Rev 2006, 86:5I5-8I.

755. Grainger DJ: TGF-beta and atherosclerosis in man. Cardiovasc Res 2007, 74:213-22.

756. Popa C, Netea MG, van Riel PL, Meer van der JW and Stalenhoef AF: The role of TNF-alpha in chronic inflammatory conditions, intermediary metabolism, and cardiovascular risk. J Lipid Res 2007, 48:75I-62.

757. Schleicher E and Friess U: Oxidative stress, AGE, and atherosclerosis. Kidney Int Suppl 2007, SI7-26.

758. Kibel A, Belovari $T$ and Drenjančević-Perić I: The role of transferrin in atherosclerosis. Med Hypotheses 2008, 70:793-7.

759. Packard RR and Libby P: Inflammation in atherosclerosis: from vascular biology to biomarker discovery and risk prediction. Clin Chem 2008, 54:24-38.

760. Rader DJ and Daugherty A: Translating molecular discoveries into new therapies for atherosclerosis. Nature 2008, 45I:904-13

76I. Tan KT and Lip GY: Imaging of the unstable plaque. Int J Cardiol 2008, I 27: I57-65.

762. van Leuven SI, Franssen R, Kastelein J], Levi M, Stroes ES and Tak PP: Systemic inflammation as a risk factor for atherothrombosis. Rheumatology (Oxford) 2008, 47:3-7.

763. Lusis AJ: Atherosclerosis. Nature 2000, 407:233-24I.

764. Stocker $R$ and Keaney JF: Role of oxidative modifications in atherosclerosis. Physiol Rev 2004, 84: |38I-1478.

765. Smith C, Mitchinson MJ, Aruoma OI and Halliwell B: Stimulation of lipid peroxidation and hydroxyl radical generation by the contents of human atherosclerotic lesions. Biochem J 1992, 286:90I-905.

766. Ramakrishna G, Rooke TW and Cooper LT: Iron and peripheral arterial disease: revisiting the iron hypothesis in a different light. Vasc Med 2003, 8:203-210.

767. Stadler N, Lindner RA and Davies MJ: Direct detection and quantification of transition metal ions in human athero- sclerotic plaques: evidence for the presence of elevated levels of iron and copper. Arterioscler Thromb Vasc Biol 2004 24:949-54.

768. Wolff B, Volzke H, Ludemann J, Robinson D, Vogelgesang D, Staudt A, Kessler C, Dahm JB, John U and Felix SB: Association between high serum ferritin levels and carotid atherosclerosis in the study of health in Pomerania (SHIP). Stroke 2004, 35:453-7.

769. Roijers RB, Dutta RK, Mutsaers PHA, Gijbels MJJ, de Winther MPJ, de Goeij JJM and Vusse van der GJ: Spatial correlation of trace elements with morphological features of atherosclerotic plaques. Nuclear Instruments \& Methods in Physics Research Section BBeam Interactions with Materials and Atoms 2005, 23 I:239-244.

770. Stocker R and Keaney JF: New insights on oxidative stress in the artery wall. Journal of Thrombosis and Haemostasis 2005, 3:1825-1834

77I. Stanley N, Stadler N, Woods AA, Bannon PG and Davies MJ: Concentrations of iron correlate with the extent of protein, but not lipid, oxidation in advanced human atherosclerotic lesions. Free Radical Biology and Medicine 2006, 40:1636-I643.

772. Watt F, Rajendran R, Ren MQ, Tan BKH and Halliwell B: A nuclear microscopy study of trace elements $\mathrm{Ca}, \mathrm{Fe}, \mathrm{Zn}$ and $\mathrm{Cu}$ in atherosclerosis. Nucl Instr Meth Phys Res B 2006, 249:646-652.

773. Fernandes de Godoy M, Takakura IT, Machado RD, Grassi LV and Nogueira PR: Serum ferritin and obstructive coronary artery disease: Angiographic correlation. Arquivos Brasileiros De Cardiologia 2007, 88:430-433.

774. Rajendran R, Ren M, Ning $P$, Tan Kwong Huat B, Halliwell B and Watt F: Promotion of atherogenesis by copper or iron-which is more likely? Biochem Biophys Res Commun 2007, 353:6-10.

775. Kazi TG, Afridi $H I$, Kazi N, Jamali MK, Arain MB, Sarfraz RA Jalbani N, Ansari R, Shah AQ, Memon AU and Khandhro GA: Distribution of zinc, copper and iron in biological samples of Pakistani myocardial infarction (Ist, 2nd and 3rd heart attack) patients and controls. Clin Chim Acta 2008, 389:।|4-9.

776. Sullivan JL: Iron in arterial plaque: A modifiable risk factor for atherosclerosis. Biochim Biophys Acta 2008 in press.

777. Yuan $\mathrm{XM}$ and $\mathrm{Li} \mathrm{W}$ : Iron involvement in multiple signaling pathways of atherosclerosis: a revisited hypothesis. Curr Med Chem 2008, 15:2157-72.

778. Matthews AJ, Vercellotti GM, Menchaca HJ, Bloch PH, Michalek VN, Marker PH, Murar J and Buchwald $\mathrm{H}$ : Iron and atherosclerosis: inhibition by the iron chelator deferiprone (LI). J Surg Res 1997, 73:35-40.

779. Ponraj D, Makjanic J, Thong PSP, Tan BKH and Watt F: The onset of atherosclerotic lesion formation in hypercholesterolemic rabbits is delayed by iron depletion. FEBS Lett 1999, 459:218-222.

780. Lee TS, Shiao MS, Pan CC and Chau LY: Iron-deficient diet reduces atherosclerotic lesions in ApoE-deficient mice. Circulation 1999, 99:1222-1229.

78I. Ren MQ, Rajendran R, Pan N, Tan BKH, Ong WY, Watt F and Halliwell $B$ : The iron chelator desferrioxamine inhibits atherosclerotic lesion development and decreases lesion iron concentrations in the cholesterol-fed rabbit. Free Radical Biology and Medicine 2005, 38:1206-1211.

782. Ferrara DE and Taylor WR: Iron chelation and vascular function: in search of the mechanisms. Arterioscler Thromb Vasc Biol 2005, 25:2235-7.

783. Kiechl S, Willeit J, Egger G, Poewe W and Oberhollenzer F: Body iron stores and the risk of carotid atherosclerosis: prospective results from the Bruneck study. Circulation I997, 96:3300-7.

784. de Valk B and Marx JJM: Iron, atherosclerosis, and ischemic heart disease. Arch Internal Med 1999, I 59: I542-I548.

785. Zacharski LR, Chow B, Lavori PW, Howes PS, Bell MR, DiTommaso MA, Carnegie NM, Bech F, Amidi M and Muluk S: The Iron (Fe) and Atherosclerosis Study (FeAST): A pilot study of reduction of body iron stores in atherosclerotic peripheral vascular disease. American Heart Journal 2000, 139:337-345.

786. Day SM, Duquaine D, Mundada LV, Menon RG, Khan BV, Rajagopalan S and Fay WP: Chronic iron administration increases vascular oxidative stress and accelerates arterial thrombosis. Circulation 2003, 107:260I-2606.

787. Ren MQ, Watt F, Huat BTK and Halliwell B: Trace elemental distributions in induced atherosclerotic lesions using nuclear microscopy. Nuclear Instr Meth Phys Res B 2003 , $210: 336-342$. 
788. Minqin R, Watt F, Huat BTK and Halliwell B: Correlation of iron and zinc levels with lesion depth in newly formed atherosclerotic lesions. Free Radical Biology and Medicine 2003, 34:746-752.

789. You SA, Archacki SR, Angheloiu G, Moravec CS, Rao S, Kinter M, Topol EJ and Wang Q: Proteomic approach to coronary atherosclerosis shows ferritin light chain as a significant marker: evidence consistent with iron hypothesis in atherosclerosis. Physiol Genomics 2003, 13:25-30.

790. Zacharski LR and Gerhard GS: Atherosclerosis: a manifestation of chronic iron toxicity? Vascular Medicine 2003, 8: I53-I55.

791. Kallianpur AR: Iron and oxidative injury - A commentary on "Fatty acid-mediated iron translocation: A synergistic mechanism of oxidative injury" by D. Yao et al. Free Radical Biology and Medicine 2005, 39:1305-1309.

792. You SA and Wang Q: Ferritin in atherosclerosis. Clin Chim Acta 2005, 357:1-16.

793. Lapenna D, Pierdomenico SD, Ciofani G, Ucchino S, Neri M, Giamberardino MA and Cuccurullo F: Association of body iron stores with low molecular weight iron and oxidant damage of human atherosclerotic plaques. Free Rad Biol Med 2007, 42:492-498.

794. Marx J], Kartikasari AE and Georgiou NA: Can iron chelators influence the progression of atherosclerosis? Hemoglobin 2008, 32: $123-34$.

795. Paulsson J, Dadfar E, Held C, Jacobson SH and Lundahl J: Activation of peripheral and in vivo transmigrated neutrophils in patients with stable coronary artery disease. Atherosclerosis 2007, 1 92:328-34.

796. Rooyakkers TM, Stroes ES, Kooistra MP, van Faassen EE, Hider RC, Rabelink TJ and Marx J): Ferric saccharate induces oxygen radical stress and endothelial dysfunction in vivo. Eur $J$ Clin Invest 2002, 32(Suppl I):9-16.

797. Duffy SJ, Biegelsen ES, Holbrook M, Russell JD, Gokce N, Keaney JF Jr and Vita JA: Iron chelation improves endothelial function in patients with coronary artery disease. Circulation 2001, 103:2799-804.

798. Ishizaka N, Saito K, Mori I, Matsuzaki G, Ohno M and Nagai R: Iron chelation suppresses ferritin upregulation and attenuates vascular dysfunction in the aorta of angiotensin II-infused rats. Arterioscler Thromb Vasc Biol 2005, 25:2282-8.

799. Saito K, Ishizaka N, Aizawa T, Sata M, Iso-o N, Noiri E, Mori I, Ohno $M$ and Nagai $R$ : Iron chelation and a free radical scavenger suppress angiotensin II-induced upregulation of TGF-betal in the heart. Am J Physiol Heart Circ Physiol 2005, 288: HI836-43.

800. Thomas SR, Schulz E and Keaney JF: Hydrogen peroxide restrains endothelium-derived nitric oxide bioactivity Role for iron-dependent oxidative stress. Free Radical Biology and Medicine 2006, 41:681-688.

80I. Zacharski LR, Chow BK, Howes PS, Shamayeva G, Baron JA, Dalman RL, Malenka DJ, Ozaki CK and Lavori PW: Reduction of iron stores and cardiovascular outcomes in patients with peripheral arterial disease - A randomized controlled trial. JAMA 2007, 297:603-6I0.

802. Shiesh SC, Chen CY, Lin XZ, Liu ZA and Tsao HC: Melatonin prevents pigment gallstone formation induced by bile duct ligation in guinea pigs. Hepatology 2000, 32:455-60.

803. Koppisetti S, Jenigiri B, Terron MP, Tengattini S, Tamura H, Flores LJ, Tan DX and Reiter RJ: Reactive oxygen species and the hypomotility of the gall bladder as targets for the treatment of gallstones with melatonin: a review. Dig Dis Sci 2008 , 53:2592-603.

804. Robinson JG: Models for describing relations among the various statin drugs, low-density lipoprotein cholesterol lowering, pleiotropic effects, and cardiovascular risk. Am Cardiol 2008, I01:1009-15.

805. Prentice RL, Langer RD, Stefanick ML, Howard BV, Pettinger M, Anderson GL, Barad D, Curb JD, Kotchen J, Kuller L, Limacher M and Wactawski-Wende J: Combined analysis of Women's Health Initiative observational and clinical trial data on postmenopausal hormone treatment and cardiovascular disease. Am J Epidemiol 2006, 163:589-99.

806. Couzin J: Cholesterol veers off script. Science 2008, 322:220-3.

807. Peterson RT: Chemical biology and the limits of reductionism. Nat Chem Biol 2008, 4:635-8.

808. Weitz-Schmidt G, Welzenbach K, Brinkmann V, Kamata T, Kallen J, Bruns C, Cottens S, Takada $Y$ and Hommel U: Statins selectively inhibit leukocyte function antigen-I by binding to a novel regulatory integrin site. Nat Med 2001, 7:687-92.
809. Arkin MR and Wells JA: Small-molecule inhibitors of proteinprotein interactions: progressing towards the dream. Nat Rev Drug Discov 2004, 3:301-I7.

810. Bellosta S, Ferri N, Arnaboldi L, Bernini F, Paoletti R and Corsini A: Pleiotropic effects of statins in atherosclerosis and diabetes. Diabetes Care 2000, 23:B72-U5.

8II. Kwak B, Mulhaupt F, Myit S and Mach F: Statins as a newly recognized type of immunomodulator. Nat Med 2000, 6:1399-402.

812. Albert MA, Danielson E, Rifai $N$ and Ridker PM: Effect of statin therapy on C-reactive protein levels: the pravastatin inflammation/CRP evaluation (PRINCE): a randomized trial and cohort study. JAMA 200I, 286:64-70.

8I3. LaRosa JC: Pleiotropic effects of statins and their clinical significance. American Journal of Cardiology 200I, 88:29I.

8I4. Takemoto $M$, Node K, Nakagami H, Liao YL, Grimm M, Takemoto $Y$, Kitakaze $M$ and Liao JK: Statins as antioxidant therapy for preventing cardiac myocyte hypertrophy. Journal of Clinical Investigation 2001, 108: |429-|437.

8I5. Liao JK: Beyond lipid lowering: the role of statins in vascular protection. Int J Cardiol 2002, 86:5-18.

816. McFarlane SI, Muniyappa R, Francisco R and Sowers JR: Clinica review I45 - Pleiotropic effects of statins: Lipid reduction and beyond. J Clin Endocrinol Metab 2002, 87:|45|-|458.

817. Undas A, Brozek J and Musial J: Anti-inflammatory and antithrombotic effects of statins in the management of coronary artery disease. Clin Lab 2002, 48:287-96.

818. Bonetti PO, Lerman LO, Napoli C and Lerman A: Statin effects beyond lipid lowering-are they clinically relevant? Eur Heart J 2003, 24:225-48.

819. Dichtl W, Dulak J, Frick M, Alber HF, Schwarzacher SP, Ares MPS, Nilsson J, Pachinger $O$ and Weidinger F: HMG-CoA reductase inhibitors regulate inflammatory transcription factors in human endothelial and vascular smooth muscle cells. Arteriosclerosis Thrombosis and Vascular Biology 2003, 23:58-63.

820. Mulhaupt F, Matter CM, Kwak BR, Pelli G, Veillard NR, Burger F, Graber P, Luscher TF and Mach F: Statins (HMG-CoA reductase inhibitors) reduce CD40 expression in human vascular cells. Cardiovasc Res 2003, 59:755-66.

821. Wierzbicki AS, Poston R and Ferro A: The lipid and non-lipid effects of statins. Pharmacol Ther 2003, 99:95-II 2 .

822. Davignon J: Beneficial cardiovascular pleiotropic effects of statins. Circulation 2004, 109:39-43.

823. Halcox JP and Deanfield JE: Beyond the laboratory: clinical implications for statin pleiotropy. Circulation 2004, I09:I142-8.

824. Liao JK: Statins: potent vascular anti-inflammatory agents. Int I Clin Pract Suppl 2004, 4I-8.

825. Schönbeck $U$ and Libby P: Inflammation, immunity, and HMGCoA reductase inhibitors - Statins as antiinflammatory agents? Circulation 2004, 109:18-26.

826. Liao JK and Laufs U: Pleiotropic effects of statins. Annu Rev Pharmacol Toxicol 2005, 45:89-I I8.

827. Elrod JW and Lefer DJ: The effects of statins on endothelium, inflammation and cardioprotection. Drug News Perspect 2005, 18:229-36

828. Robinson JG, Smith B, Maheshwari N and Schrott H: Pleiotropic effects of statins: Benefit beyond cholesterol reduction? A meta-regression analysis. J Amer Coll Cardiol 2005, 46: I855-I 862.

829. Tsubouchi H, Inoguchi T, Sonta T, Sato N, Sekiguchi N, Kobayashi K, Sumimoto $H$, Utsumi $H$ and Nawata $H$ : Statin attenuates high glucose-induced and diabetes-induced oxidative stress in vitro and in vivo evaluated by electron spin resonance measurement. Free Radical Biology and Medicine 2005, 39:444-452.

830. Beckman JA and Creager MA: The nonlipid effects of statins on endothelial function. Trends Cardiovasc Med 2006, 16: I56-62.

831. Carloni S, Mazzoni E, Cimino M, De Simoni MG, Perego C, Scopa C and Balduini W: Simvastatin reduces caspase- 3 activation and inflammatory markers induced by hypoxia-ischemia in the newborn rat. Neurobiol Dis 2006, 2 I: I I9-26.

832. Grimes DS: Are statins analogues of vitamin D? Lancet 2006 368:83-6.

833. Paumelle R, Blanquart C, Briand O, Barbier O, Duhem C, Woerly G, Percevault F, Fruchart JC, Dombrowicz D, Glineur C and Staels B: Acute antiinflammatory properties of statins involve peroxisome proliferator-activated receptor-alpha via inhibition of the protein kinase C signaling pathway. Circ Res 2006, 98:36I-9.

834. Alegret $M$ and Silvestre JS: Pleiotropic effects of statins and related pharmacological experimental approaches. Timely Top Med Cardiovasc Dis 2007, I I:EIO. 
835. Farooqui AA, Ong WY, Horrocks LA, Chen $P$ and Farooqui $T$ : Comparison of biochemical effects of statins and fish oil in brain: the battle of the titans. Brain Res Rev 2007, 56:443-7I.

836. Kuipers HF and Elsen van den PJ: Immunomodulation by statins: inhibition of cholesterol vs. isoprenoid biosynthesis. Biomed Pharmacother 2007, 6 I:400-7.

837. Lahera V, Goicoechea M, de Vinuesa SG, Miana M, de las Heras N, Cachofeiro $V$ and Luno J: Endothelial dysfunction, oxidative stress and inflammation in atherosclerosis: beneficial effects of statins. Curr Med Chem 2007, 14:243-8.

838. Paraskevas KI, Tzovaras AA, Briana DD and Mikhailidis DP: Emerging indications for statins: a pluripotent family of agents with several potential applications. Curr Pharm Des 2007, 13:3622-36.

839. Terblanche M, Almog Y, Rosenson R, Smith TS and Hackam DG: Statins and sepsis: multiple modifications at multiple levels. Lancet Infectious Diseases 2007, 7:358-368.

840. Wang CY, Liu PY and Liao JK: Pleiotropic effects of statin therapy: molecular mechanisms and clinical results. Trends Mol Med 2008, I 4:37-44.

84I. Wagner BK, Kitami T, Gilbert TJ, Peck D, Ramanathan A, Schreiber SL, Golub TR and Mootha VK: Large-scale chemical dissection of mitochondrial function. Nat Biotechnol 2008, 26:343-351.

842. Libby $P$ and Aikawa M: Stabilization of atherosclerotic plaques: new mechanisms and clinical targets. Nat Med 2002, 8: $1257-62$.

843. Blake GJ and Ridker PM: Are statins anti-inflammatory? Curr Control Trials Cardiovasc Med 2000, I:I6I-165.

844. Diomede L, Albani D, Sottocorno M, Donati MB, Bianchi $M$, Fruscella $P$ and Salmona $M$ : In vivo anti-inflammatory effect of statins is mediated by nonsterol mevalonate products. Arterioscler Thromb Vasc Biol 2001, 2 I: I 327-32.

845. Laufs U, Gertz K, Dirnagl U, Bohm M, Nickenig G and Endres M: Rosuvastatin, a new HMG-CoA reductase inhibitor, upregulates endothelial nitric oxide synthase and protects from ischemic stroke in mice. Brain Res 2002, 942:23-30.

846. Neuhaus O, Strasser-Fuchs S, Fazekas F, Kieseier BC, Niederwieser G, Hartung HP and Archelos J]: Statins as immunomodulators: comparison with interferon-beta Ib in MS. Neurology 2002, 59:990-7.

847. Rasmussen LM, Hansen PR, Nabipour MT, Olesen P, Kristiansen MT and Ledet $T$ : Diverse effects of inhibition of 3-hydroxy-3. methylglutaryl-CoA reductase on the expression of VCAMI and E-selectin in endothelial cells. Biochem J 200I, 360:363-70

848. Takemoto $M$ and Liao JK: Pleiotropic effects of 3-hydroxy-3methylglutaryl coenzyme a reductase inhibitors. Arterioscler Thromb Vasc Biol 2001, 21: I7I2-9.

849. Sukhova GK, Williams JK and Libby P: Statins reduce inflammation in atheroma of nonhuman primates independent of effects on serum cholesterol. Arterioscler Thromb Vasc Biol 2002, 22: 1452-8.

850. Scalia R and Stalker TJ: Microcirculation as a target for the antiinflammatory properties of statins. Microcirculation 2002, 9:431-42.

85I. Weitz-Schmidt G: Statins as anti-inflammatory agents. Trends Pharmacol Sci 2002, 23:482-6.

852. Youssef S, Stuve O, Patarroyo JC, Ruiz PJ, Radosevich JL, Hur EM, Bravo M, Mitchell DJ, Sobel RA, Steinman L and Zamvil SS: The HMG-CoA reductase inhibitor, atorvastatin, promotes a Th2 bias and reverses paralysis in central nervous system autoimmune disease. Nature 2002, 420:78-84.

853. Balk EM, Lau J, Goudas LC, Jordan HS, Kupelnick B, Kim LU and Karas RH: Effects of statins on nonlipid serum markers associated with cardiovascular disease: a systematic review. Ann Intern Med 2003, 139:670-82.

854. Blanco-Colio LM, Tuñon J, Martin-Ventura JL and Egido J: Antiinflammatory and immunomodulatory effects of statins. Kidney Int 2003, 63:12-23.

855. Kwak BR, Mulhaupt $F$ and Mach F: Atherosclerosis: antiinflammatory and immunomodulatory activities of statins. Autoimmun Rev 2003, 2:332-8.

856. Mason IC: Statins and their role in vascular protection. Clin Sci (Lond) 2003, 105:25I-66.

857. Naidu BV, Woolley SM, Farivar AS, Thomas R, Fraga C and Mulligan MS: Simvastatin ameliorates injury in an experimental model of lung ischemia-reperfusion. J Thorac Cardiovasc Surg 2003, I 26:482-9.
858. Pate GE, Tahir MN, Murphy RT and Foley JB: Anti-inflammatory effects of statins in patients with aortic stenosis. I Cardiovasc Pharmacol Ther 2003, 8:201-6.

859. Shishehbor MH, Aviles RJ, Brennan ML, Fu XM, Goormastic M, Pearce GL, Gokce N, Keaney JF, Penn MS, Sprecher DL, Vita JA and Hazen SL: Association of nitrotyrosine levels with cardiovascular disease and modulation by statin therapy. JAMA 2003, 289: |675-|680.

860. Stüve O, Youssef S, Dunn S, Slavin AJ, Steinman L and Zamvil SS: The potential therapeutic role of statins in central nervous system autoimmune disorders. Cell Mol Life Sci 2003, 60:2483-91.

861. Stüve O, Prod'homme T, Slavin A, Youssef S, Dunn S, Steinman L and Zamvil SS: Statins and their potential targets in multiple sclerosis therapy. Expert Opin Ther Targets 2003, 7:613-22.

862. Stüve O, Youssef S, Steinman L and Zamvil SS: Statins as potential therapeutic agents in neuroinflammatory disorders. Curr Opin Neurol 2003, 16:393-401.

863. Durant R, Klouche K, Delbosc S, Morena M, Amigues L, Beraud JJ, Canaud B and Cristol JP: Superoxide anion overproduction in sepsis: effects of vitamin e and simvastatin. Shock 2004, 22:34-9.

864. Endres $M$ and Laufs U: Effects of statins on endothelium and signaling mechanisms. Stroke 2004, 35:2708-II.

865. Kunkel EJ, Dea M, Ebens A, Hytopoulos E, Melrose J, Nguyen D, Ota KS, Plavec I, Wang Y, Watson SR, Butcher EC and Berg EL: An integrative biology approach for analysis of drug action in models of human vascular inflammation. FASEB J 2004, I 8: | 279-8|.

866. McCarey DW, Mclnnes IB, Madhok R, Hampson R, Scherbakov O, Ford I, Capell HA and Sattar N: Trial of Atorvastatin in Rheumatoid Arthritis (TARA): double-blind, randomised placebo-controlled trial. Lancet 2004, 363:20 I5-2I.

867. Rosenson RS: Statins in atherosclerosis: lipid-lowering agents with antioxidant capabilities. Atherosclerosis 2004, I 73:1-12.

868. Mclnnes IB, McCarey DW and Sattar N: Do statins offer therapeutic potential in inflammatory arthritis? Ann Rheum Dis 2004, 63: I535-7.

869. Steffens S and Mach F: Anti-inflammatory properties of statins. Semin Vasc Med 2004, 4:4I7-22.

870. Stoll LL, McCormick ML, Denning GM and Weintraub NL. Antioxidant effects of statins. Drugs Today (Barc) 2004, 40:975-90.

87I. Arnaud C, Braunersreuther $V$ and Mach F: Toward immunomodulatory and anti-inflammatory properties of statins. Trends Cardiovasc Med 2005, I 5:202-6.

872. Endres M: Statins and stroke. J Cereb Blood Flow Metab 2005, 25:1093-110.

873. Gaugler MH, Vereycken-Holler V, Squiban C, Vandamme M, Vozenin-Brotons $M C$ and Benderitter $M$ : Pravastatin limits endothelial activation after irradiation and decreases the resulting inflammatory and thrombotic responses. Radiat Res 2005, 163:479-87.

874. Jain MK and Ridker PM: Anti-inflammatory effects of statins: clinical evidence and basic mechanisms. Nat Rev Drug Discov 2005, 4:977-87.

875. Macin SM, Perna ER, Farias EF, Franciosi V, Cialzeta JR, Brizuela M, Medina F, Tajer C, Doval H and Badaracco R: Atorvastatin has an important acute anti-inflammatory effect in patients with acute coronary syndrome: results of a randomized, doubleblind, placebo-controlled study. Am Heart J 2005, I49:45 I-7.

876. Methe $H$, Kim JO, Kofler S, Nabauer $M$ and Weis $M$ : Statins decrease Toll-like receptor 4 expression and downstream signaling in human CD 14+ monocytes. Arterioscler Thromb VasC Biol 2005, 25: 1439-45.

877. Schultz Johansen J, Harris AK, Rychly DJ and Ergul A: Oxidative stress and the use of antioxidants in diabetes: linking basic science to clinical practice. Cardiovasc Diabetol 2005, 4:5.

878. Tonelli M, Sacks F, Pfeffer M, Jhangri GS and Curhan G: Biomarkers of inflammation and progression of chronic kidney disease. Kidney Int 2005, 68:237-45.

879. Abeles $A M$ and Pillinger $M H$ : Statins as antiinflammatory and immunomodulatory agents: a future in rheumatologic therapy? Arthritis Rheum 2006, 54:393-407.

880. Endres $M$ : Statins: potential new indications in inflammatory conditions. Atheroscler Suppl 2006, 7:31-5.

88I. Yang J, Li XP, Zhao SP, Li J, Li JD and Xie XM: The effect of different doses of fluvastatin on inflammatory markers in the early phase of acute coronary syndrome. Clin Chim Acto 2006, 368: 183-7. 
882. Ahn KS, Sethi G and Aggarwal BB: Simvastatin potentiates TNFalpha-induced apoptosis through the down-regulation of NF-kappaB-dependent antiapoptotic gene products: role of IkappaBalpha kinase and TGF-beta-activated kinase-I. J Immunol 2007, I 78:2507-16.

883. Becker RC: Off-target properties of pharmacotherapy and the importance of mechanistic investigations in early clinical phase drug development. I Thromb Thrombolysis 2007, 23: I59-6I .

884. Greenwood J and Mason JC: Statins and the vascular endothelial inflammatory response. Trends Immunol 2007, 28:88-98.

885. Li JJ, Zheng $X$ and $\mathrm{Li} \mathrm{J:} \mathrm{Statins} \mathrm{may} \mathrm{be} \mathrm{beneficial} \mathrm{for} \mathrm{patients}$ with slow coronary flow syndrome due to its anti-inflammatory property. Med Hypotheses 2007, 69:333-7.

886. Sandek A, Utchill S and Rauchhaus M: The endotoxin-lipoprotein hypothesis - an update. Archives of Medical Science 2007, 3: S8I-S90.

887. Siffrin V, Brandt AU, Herz J and Zipp F: New insights into adaptive immunity in chronic neuroinflammation. Adv Immunol 2007, 96: I-40.

888. Souza-Costa DC, Sandrim VC, Lopes LF, Gerlach RF, Rego EM and Tanus-Santos JE: Anti-inflammatory effects of atorvastatin: modulation by the T-786C polymorphism in the endothelial nitric oxide synthase gene. Atherosclerosis 2007, 193:438-44.

889. Hernández-Romero MdC, Argüelles S, Villaran RF, de Pablos RM, Delgado-Cortés MJ, Santiago M, Herrera AJ, Cano J and Machado A: Simvastatin prevents the inflammatory process and the dopaminergic degeneration induced by the intranigral injection of lipopolysaccharide. I Neurochem 2008, I 05:445-59.

890. Paraskevas KI: Statin treatment for rheumatoid arthritis: a promising novel indication. Clin Rheumatol 2008, 27:28I-7.

89l. Prinz V, Laufs U, Gertz K, Kronenberg G, Balkaya M, Leithner C, Lindauer $U$ and Endres $M$ : Intravenous rosuvastatin for acute stroke treatment: an animal study. Stroke 2008, 39:433-8.

892. Xu SZ, Zhong W, Watson NM, Dickerson E, Wake JD, Lindow SW, Newton CJ and Atkin SL: Fluvastatin reduces oxidative damage in human vascular endothelial cells by upregulating Bcl-2. J Thromb Haemost 2008, 6:692-700.

893. Dombrecht EJ, De Tollenaere CB, Aerts K, Cos P, Schuerwegh AJ, Bridts CH, Van Offel JF, Ebo DG, Stevens WJ and De Clerck LS: Antioxidant effect of bisphosphonates and simvastatin on chondrocyte lipid peroxidation. Biochem Biophys Res Commun 2006, 348:459-64.

894. Lee DH, Zacharski LR and Jacobs DR: Comparison of the serum ferritin and percentage of transferrin saturation as exposure markers of iron-driven oxidative stress-related disease outcomes. American Heart Journal 2006, I 5 I:.

895. Anderson LJ, Holden S, Davis B, Prescott E, Charrier CC, Bunce NH, Firmin DN, Wonke B, Porter J, Walker JM and Pennell DJ: Cardiovascular T2-star (T2*) magnetic resonance for the early diagnosis of myocardial iron overload. Eur Heart J 200I,

896. Yeoh-Ellerton S and Stacey MC: Iron and 8-isoprostane levels in acute and chronic wounds. J Invest Dermatol 2003, I 21:918-925.

897. Bergan J, Schmid-Schonbein GW, Smith PDC, Nicolaides AN, Boisseau MR and Eklof B: Mechanisms of disease: Chronic venous disease. New England Journal of Medicine 2006, 355:488-498.

898. Zamboni P, Izzo M, Fogato L, Carandina S and Lanzara V: Urine hemosiderin: a novel marker to assess the severity of chronic venous disease. J Vasc Surg 2003, 37:132-6.

899. Zamboni P: The Big Idea: Iron-dependent inflammation in venous disease and proposed parallels in multiple sclerosis. J R Soc Med 2006, 99:589-593.

900. Lucas SM, Rothwell NJ and Gibson RM: The role of inflammation in CNS injury and disease. Br J Pharmacol 2006, I47(Suppl I): S232-40.

901. Lewén $\mathrm{A}$, Matz $\mathrm{P}$ and $\mathrm{Chan} \mathrm{PH}$ : Free radical pathways in CNS injury. J Neurotrauma 2000, I 7:87|-90.

902. Que EL, Domaille DW and Chang CJ: Metals in neurobiology: probing their chemistry and biology with molecular imaging. Chem Rev 2008, 108:1517-49.

903. Kent TA, Soukup VM and Fabian RH: Heterogeneity affecting outcome from acute stroke therapy: making reperfusion worse. Stroke 200I, 32:2318-27.

904. Emsley HC and Tyrrell PJ: Inflammation and infection in clinical stroke. J Cereb Blood Flow Metab 2002, 22:1399-419.

905. Patt A, Horesh IR, Berger EM, Harken AH and Repine JE: Iron depletion or chelation reduces ischemia/reperfusioninduced edema in gerbil brains. J Pediatr Surg 1990, 25:224-7.
906. Dávalos A, Fernandezreal JM, Ricart W, Soler S, Molins A, Planas E and Genis D: Iron-related damage in acute ischemic stroke. Stroke 1994, 25:1543-1546.

907. Bishop GM and Robinson SR: Quantitative analysis of cell death and ferritin expression in response to cortical iron: implications for hypoxia-ischemia and stroke. Brain Research 200I, 907:175-187.

908. Demougeot C, Van Hoecke M, Bertrand N, Prigent-Tessier A Mossiat C, Beley A and Marie C: Cytoprotective efficacy and mechanisms of the liposoluble iron chelator 2,2 '-dipyridyl in the rat photothrombotic ischemic stroke model. J Pharm Exp Therapeut 2004, 3 I I: 1080-1087

909. Mehta SH, Webb RC, Ergul A, Tawak A and Dorrance AM: Neuroprotection by tempol in a model of iron-induced oxidative stress in acute ischemic stroke. Am J Physiol 2004, 286:R283-R288.

910. Selim $\mathrm{MH}$ and Ratan RR: The role of iron neurotoxicity in ischemic stroke. Ageing Research Reviews 2004, 3:345-353.

911. van der ADL, Grobbee DE, Roest M, Marx JJM, Voorbij HA and Schouw van der YT: Serum ferritin is a risk factor for stroke in postmenopausal women. Stroke 2005, 36: I637-|64I.

912. Hua $Y$, Keep RF, Hoff $J T$ and $X i$ G: Brain injury after intracerebral hemorrhage: the role of thrombin and iron. Stroke 2007, 38:759-62.

913. Millan M, Sobrino T, Castellanos M, Nombela F, Arenillas JF, Riva E, Cristobo I, Garcia MM, Vivancos J, Serena J, Moro MA, Castillo J and Dávalos A: Increased body iron stores are associated with poor outcome after thrombolytic treatment in acute stroke. Stroke 2007, 38:90-95.

914. Slemmer JE, Shacka JJ, Sweeney MI and Weber JT: Antioxidants and free radical scavengers for the treatment of stroke, traumatic brain injury and aging. Curr Med Chem 2008 I5:404-14

915. Baker K, Marcus CB, Huffman K, Kruk H, Malfroy B and Doctrow SR: Synthetic combined superoxide dismutase/ catalase mimetics are protective as a delayed treatment in a rat stroke model: a key role for reactive oxygen species in ischemic brain injury. J Pharmacol Exp Ther 1998, 284:2 I5-2I.

916. Sun $A Y$ and Chen $Y M$ : Oxidative stress and neurodegenerative disorders. Journal of Biomedical Science 1998, 5:40I-4I4.

917. Pong K: Oxidative stress in neurodegenerative diseases: therapeutic implications for superoxide dismutase mimetics. Expert Opinion on Biological Therapy 2003, 3:127-139.

918. Bastianetto $S$ and Quirion R: Natural antioxidants and neurodegenerative diseases. Frontiers in Bioscience 2004, 9:3447-3452.

919. Warner DS, Sheng HX and Batinic-Haberle I: Oxidants, antioxidants and the ischemic brain. J Exp Biol 2004, 207:322I-323I.

920. Craft JM, Watterson DM and Van Eldik LJ: Neuroinflammation: a potential therapeutic target. Expert Opin Ther Targets 2005 , 9:887-900.

921. Margaill I, Plotkine $M$ and Lerouet D: Antioxidant strategies in the treatment of stroke. Free Radical Biology and Medicine 2005, 39:429-443.

922. Zhao BL: Natural antioxidants for neurodegenerative diseases. Mol Neurobiol 2005, 3 I:283-293.

923. Obrenovitch TP: Molecular physiology of preconditioninginduced brain tolerance to ischemia. Physiol Rev 2008 , 88:2 I I-47.

924. Sirén AL, Fratelli M, Brines M, Goemans C, Casagrande S, Lewczuk P, Keenan S, Gleiter C, Pasquali C, Capobianco A, Mennini T, Heumann R, Cerami A, Ehrenreich $\mathrm{H}$ and Ghezzi P: Erythropoietin prevents neuronal apoptosis after cerebral ischemia and metabolic stress. Proc Natl Acad Sci USA 2001, 98:4044-9.

925. Brines ML, Ghezzi P, Keenan S, Agnello D, de Lanerolle NC, Cerami C, Itri LM and Cerami A: Erythropoietin crosses the blood-brain barrier to protect against experimental brain injury. Proc Natl Acad Sci USA 2000, 97:|0526-31.

926. Digicaylioglu $M$ and Lipton SA: Erythropoietin-mediated neuroprotection involves cross-talk between Jak2 and NF-kappaB signalling cascades. Nature 200 I, 4I 2:64I-7.

927. Dawson TM: Preconditioning-mediated neuroprotection through erythropoietin? Lancet 2002, 359:96-7.

928. Ehrenreich H, Hasselblatt M, Dembowski C Cepek L, Lewczuk P Stiefel M, Rustenbeck HH, Breiter N, Jacob S, Knerlich F, Bohn M, Poser W, Ruther E, Kochen M, Gefeller O, Gleiter C, Wessel TC, De Ryck $M$, Itri L, Prange $H$, Cerami $A$, Brines $M$ and Sirén AL: Erythropoietin therapy for acute stroke is both safe and beneficial. Mol Med 2002, 8:495-505. 
929. Leker RR and Shohami E: Cerebral ischemia and traumadifferent etiologies yet similar mechanisms: neuroprotective opportunities. Brain Res Rev 2002, 39:55-73.

930. Aydin A, Genc K, Akhisaroglu M, Yorukoglu K, Gokmen N and Gonullu E: Erythropoietin exerts neuroprotective effect in neonatal rat model of hypoxic-ischemic brain injury. Brain Dev 2003, 25:494-8.

931. Kumral A, Ozer E, Yilmaz O, Akhisaroglu M, Gokmen N, Duman N, Ulukus C, Genc S and Ozkan H: Neuroprotective effect of erythropoietin on hypoxic-ischemic brain injury in neonatal rats. Biol Neonate 2003, 83:224-8.

932. Matsushita $\mathrm{H}$, Johnston MV, Lange MS and Wilson MA: Protective effect of erythropoietin in neonatal hypoxic ischemia in mice. Neuroreport 2003, |4:|757-6|.

933. Grasso G, Sfacteria A, Cerami A and Brines M: Erythropoietin as a tissue-protective cytokine in brain injury: what do we know and where do we go? Neuroscientist 2004, 10:93-8.

934. Sun $Y$, Zhou C, Polk P, Nanda A and Zhang JH: Mechanisms of erythropoietin-induced brain protection in neonatal hypoxia-ischemia rat model. J Cereb Blood Flow Metab 2004, 24:259-70.

935. Wang CH, Liang CL, Huang LT, Liu JK, Hung PH, Sun A and Hung KS: Single intravenous injection of naked plasmid DNA encoding erythropoietin provides neuroprotection in hypoxiaischemia rats. Biochem Biophys Res Commun 2004, 3 I 4: I 064-7I.

936. Bailey DM, Robach P, Thomsen JJ and Lundby C: Erythropoietin depletes iron stores: antioxidant neuroprotection for ischemic stroke? Stroke 2006, 37:2453.

937. Grasso G, Sfacteria A, Meli F, Passalacqua M, Fodale V, Buemi M, Giambartino F, lacopino DG and Tomasello $F$ : The role of erythropoietin in neuroprotection: therapeutic perspectives. Drug News Perspect 2007, 20:315-20.

938. McPherson RJ and Juul SE: Recent trends in erythropoietinmediated neuroprotection. Int I Dev Neurosci 2008, 26:103-1 I.

939. Sola $A$, Peng $H$, Rogido $M$ and Wen TC: Animal models of neonatal stroke and response to erythropoietin and cardiotrophin-I. Int J Dev Neurosci 2008, 26:27-35.

940. Erbayraktar S, Yilmaz O, Gokmen N and Brines M: Erythropoietin is a multifunctional tissue-protective cytokine. Curr Hematol Rep 2003, 2:465-70.

94I. Leist M, Ghezzi P, Grasso G, Bianchi R, Villa P, Fratelli M, Savino C, Bianchi M, Nielsen J, Gerwien J, Kallunki P, Larsen AK, Helboe L, Christensen S, Pedersen LO, Nielsen M, Torup L, Sager T, Sfacteria A, Erbayraktar S, Erbayraktar Z, Gokmen N, Yilmaz O, Cerami-Hand C, Xie QW, Coleman T, Cerami A and Brines M: Derivatives of erythropoietin that are tissue protective but not erythropoietic. Science 2004, 305:239-42.

942. Arcasoy MO: The non-haematopoietic biological effects of erythropoietin. Br J Haematol 2008, |4|:|4-3|.

943. Agnello D, Bigini P, Villa P, Mennini T, Cerami A, Brines ML and Ghezzi P: Erythropoietin exerts an anti-inflammatory effect on the CNS in a model of experimental autoimmune encephalomyelitis. Brain Res 2002, 952: I28-34.

944. Fahn S and Cohen G: The oxidant stress hypothesis in Parkinson's disease: evidence supporting it. Ann Neurol 1992, 32:804-12.

945. Halliwell B: Reactive oxygen species and the central nervous system. I Neurochem 1992, 59:1609-1623.

946. Coyle JT and Puttfarcken P: Oxidative stress, glutamate, and neurodegenerative disorders. Science 1993, 262:689-95.

947. Behl C, Davis JB, Lesley R and Schubert D: Hydrogen peroxide mediates amyloid beta protein toxicity. Cell 1994, 77:817-27.

948. Good PF, Werner P, Hsu A, Olanow CW and Perl DP: Evidence of neuronal oxidative damage in Alzheimer's disease. $\mathrm{Am} J$ Pathol 1996, 149:21-8.

949. Jenner $P$ and Olanow CW: Oxidative stress and the pathogenesis of Parkinson's disease. Neurology 1996, 47:SI6I-70.

950. Simonian NA and Coyle JT: Oxidative stress in neurodegenerative diseases. Annu Rev Pharmacol Toxicol 1996, 36:83-106.

95I. Yoritaka A, Hattori N, Uchida K, Tanaka M, Stadtman ER and Mizuno Y: Immunohistochemical detection of 4-hydroxynonenal protein adducts in Parkinson disease. Proc Natl Acad Sci USA 1996, 93:2696-701.

952. Packer L, Tritschler HJ and Wessel K: Neuroprotection by the metabolic antioxidant alpha-lipoic acid. Free Radic Biol Med 1997, 22:359-78.

953. Gao HM, Liu B, Zhang W and Hong JS: Novel anti-inflammatory therapy for Parkinson's disease. Trends Pharmacol Sci 2003, 24:395-40I.
954. Mariani E, Polidori MC, Cherubini A and Mecocci P: Oxidative stress in brain aging, neurodegenerative and vascular diseases: an overview. J Chromatogr B Analyt Technol Biomed Life Sci 2005, 827:65-75.

955. Zeevalk GD, Bernard LP, Song C, Gluck $M$ and Ehrhart I: Mitochondrial inhibition and oxidative stress: reciprocating players in neurodegeneration. Antioxid Redox Signal 2005, 7: I I 17-39.

956. Lin MT and Beal MF: Mitochondrial dysfunction and oxidative stress in neurodegenerative diseases. Nature 2006, 443:787-95.

957. Trushina E and McMurray CT: Oxidative stress and mitochondrial dysfunction in neurodegenerative diseases. Neuroscience 2007, | 45: | 233-48.

958. Benzi $G$ and Moretti $A$ : Are reactive oxygen species involved in Alzheimer's disease? Neurobiol Aging 1995, I6:66 I-74.

959. Hensley K, Hall N, Subramaniam R, Cole P, Harris M, Aksenov M, Aksenova M, Gabbita SP, Wu JF and Carney JM, et al: Brain regional correspondence between Alzheimer's disease histopathology and biomarkers of protein oxidation. J Neurochem 1995, 65:2146-56.

960. Markesbery WR: Oxidative stress hypothesis in Alzheimer's disease. Free Radic Biol Med 1997, 23:134-47.

96I. Markesbery WR and Lovell MA: 4-hydroxynonenal, a product of lipid peroxidation, is increased in the brain in Alzheimer's disease. Neurobiol Aging 1998, 19:33-6.

962. Cassarino DS and Bennett JP Jr: An evaluation of the role of mitochondria in neurodegenerative diseases: mitochondrial mutations and oxidative pathology, protective nuclear responses, and cell death in neurodegeneration. Brain Res Brain Res Rev 1999, 29: I-25.

963. Akiyama H, Barger S, Barnum S, Bradt B, Bauer J, Cole GM, Cooper NR, Eikelenboom P, Emmerling M, Fiebich BL, Finch CE, Frautschy S, Griffin WS, Hampel H, Hull M, Landreth G, Lue L, Mrak R, Mackenzie IR, McGeer PL, O'Banion MK, Pachter J, Pasinetti G, Plata-Salaman C, Rogers J, Rydel R, Shen Y, Streit W, Strohmeyer R, Tooyoma I, Van Muiswinkel FL, Veerhuis R, Walker D, Webster S, Wegrzyniak B, Wenk G and WyssCoray T: Inflammation and Alzheimer's disease. Neurobiol Aging 2000, 21:383-421.

964. Nunomura A, Perry G Aliev G Hirai K, Takeda A, Balraj EK, Jones PK, Ghanbari H, Wataya T, Shimohama S, Chiba S, Atwood CS, Petersen RB and Smith MA: Oxidative damage is the earliest event in Alzheimer disease. J Neuropathol Exp Neurol 200I, 60:759-767.

965. McGeer PL and McGeer EG: Inflammation, autotoxicity and Alzheimer disease. Neurobiol Aging 2001, 22:799-809.

966. Butterfield DA and Lauderback CM: Lipid peroxidation and protein oxidation in Alzheimer's disease brain: potential causes and consequences involving amyloid beta-peptideassociated free radical oxidative stress. Free Radic Biol Med 2002, 32: 1050-60.

967. Eikelenboom P, Bate C, Van Gool WA, Hoozemans JJ, Rozemuller JM, Veerhuis R and Williams A: Neuroinflammation in Alzheimer's disease and prion disease. Glia 2002, 40:232-9.

968. Suh YH and Checler F: Amyloid precursor protein, presenilins, and alpha-synuclein: molecular pathogenesis and pharmacological applications in Alzheimer's disease. Pharmacol Rev 2002, 54:469-525

969. McGeer EG and McGeer PL: Inflammatory processes in Alzheimer's disease. Prog Neuropsychopharmacol Biol Psychiatry 2003, 27:74I-9.

970. Butterfield DA, Perluigi $M$ and Sultana R: Oxidative stress in Alzheimer's disease brain: new insights from redox proteomics. Eur J Pharmacol 2006, 545:39-50.

971. Jenner P: Oxidative stress in Parkinson's disease. Ann Neurol 2003, 53(Suppl 3):S26-36.

972. Nunomura A, Castellani RJ, Zhu X, Moreira PI, Perry G and Smith MA: Involvement of oxidative stress in Alzheimer disease. J Neuropathol Exp Neurol 2006, 65:63I-4I.

973. Licastro F, Porcellini E, Caruso C, Lio D and Corder EH: Genetic risk profiles for Alzheimer's disease: integration of APOE genotype and variants that up-regulate inflammation. Neurobiol Aging 2007, 28: 1637-43.

974. Meyer-Luehmann M, Spires-Jones TL, Prada C, Garcia-Alloza M, de Calignon A, Rozkalne A, Koenigsknecht-Talboo J, Holtzman DM, Bacskai BJ and Hyman BT: Rapid appearance and local toxicity of amyloid-beta plaques in a mouse model of Alzheimer's disease. Nature 2008, 45 I:720-4. 
975. Goodman L: Alzheimer's disease; a clinico-pathologic analysis of twenty-three cases with a theory on pathogenesis. J Nerv Ment Dis 1953, I I 8:97-130.

976. Dexter DT, Wells FR, Lees AJ, Agid F, Agid $Y$, Jenner $P$ and Marsden CD: Increased nigral iron content and alterations in other metal ions occurring in brain in Parkinsons disease. J Neurochem 1989, 52:1830-1836.

977. Riederer P, Sofic E, Rausch WD, Schmidt B, Reynolds GP, Jellinger K and Youdim MB: Transition metals, ferritin, glutathione, and ascorbic acid in parkinsonian brains. J Neurochem 1989, 52:515-20.

978. Jellinger K, Paulus W, Grundke-lqbal I, Riederer P and Youdim MB: Brain iron and ferritin in Parkinson's and Alzheimer's diseases. J Neural Transm Park Dis Dement Sect 1990, 2:327-40.

979. O'Brien PJ: Molecular mechanisms of quinone cytotoxicity. Chemico-Biol Interact I99I, 80:I-4I.

980. Connor JR, Snyder BS, Beard JL, Fine RE and Mufson EJ: Regional distribution of iron and iron-regulatory proteins in the brain in aging and Alzheimers disease. I Neuroscie Res 1992, 31:327-335.

981. Good PF, Perl DP, Bierer LM and Schmeidler J: Selective accumulation of aluminum and iron in the neurofibrillary tangles of Alzheimer's disease: a laser microprobe (LAMMA) study. Ann Neurol 1992, 31:286-92.

982. Montgomery EB Jr: Heavy metals and the etiology of Parkinson's disease and other movement disorders. Toxicology 1995, 97:3-9.

983. Koeppen $\mathrm{AH}$ : The history of iron in the brain. J Neurol Sci I995, I 34(Suppl): I-9.

984. Deibel MA, Ehmann WD and Markesbery WR: Copper, iron, and zinc imbalances in severely degenerated brain regions in Alzheimer's disease: possible relation to oxidative stress. J Neurol Sci 1996, I43: I37-42.

985. Gorell JM, Johnson CC, Rybicki BA, Peterson EL, Kortsha GX, Brown GG and Richardson RJ: Occupational exposures to metals as risk factors for Parkinson's disease. Neurology 1997, 48:650-8.

986. Koeppen $\mathrm{AH}$ : A brief history of brain iron research. J Neurol Sci 2003, 207:95-7.

987. Youdim $\mathrm{MBH}$, Benshachar $\mathrm{D}$ and Riederer $\mathrm{P}$ : The possible role of iron in the etiopathology of Parkinsons disease. Movement Disorders 1993, 8: I-12

988. Gerlach $M$, Benshachar D, Riederer $\mathrm{P}$ and Youdim $\mathrm{MBH}$ : Altered brain metabolism of iron as a cause of neurodegenerative diseases. I Neurochem 1994, 63:793-807.

989. Götz ME, Künig G, Riederer P and Youdim MBH: Oxidative stress - free radical production in neural degeneration. Pharmacol Therapeut 1994, 63:37-122.

990. Smith MA, Perry G, Richey PL, Sayre LM, Anderson VE, Beal MF and Kowall N: Oxidative damage in Alzheimer's. Nature 1996, 382: $|20-12|$.

991. LeVine SM: Iron deposits in multiple sclerosis and Alzheimer's disease brains. Brain Research 1997, 760:298-303.

992. Smith MA, Harris PLR, Sayre LM and Perry G: Iron accumulation in Alzheimer disease is a source of redox-generated free radicals. Proc Natl Acad Sci 1997, 94:9866-9868.

993. Lovell MA, Robertson JD, Teesdale WJ, Campbell JL and Markesbery WR: Copper, iron and zinc in Alzheimer's disease senile plaques. J Neurol Sci 1998, I 58:47-52.

994. Bartzokis G, Cummings J, Perlman S, Hance DB and Mintz J: Increased basal ganglia iron levels in Huntington disease. Arch Neurol 1999, 56:569-74.

995. Huang XD, Cuajungco MP, Atwood CS, Hartshorn MA, Tyndall JDA Hanson GR, Stokes KC, Leopold M, Multhaup G, Goldstein LE, Scarpa RC, Saunders AJ, Lim J, Moir RD, Glabe C, Bowden EF, Masters CL, Fairlie DP, Tanzi RE and Bush Al: Cu(II) potentiation of Alzheimer A beta neurotoxicity - Correlation with cellfree hydrogen peroxide production and metal reduction. J Biol Chem 1999, 274:371 II-371 I6.

996. Jellinger KA: The role of iron in neurodegeneration Prospects for pharmacotherapy of Parkinson's disease. Drugs \& Aging 1999, 14:115-|40.

997. Olanow CW and Tatton WG: Etiology and pathogenesis of Parkinson's disease. Annu Rev Neurosci 1999, 22:123-i 44.

998. Sayre LM, Perry G and Smith MA: Redox metals and neurodegenerative disease. Current Opinion in Chemical Biology I999, 3:220-225.

999. Bush Al: Metals and neuroscience. Curr Opin Chem Biol 2000, 4:|84-9|.
1000. Chiueh CC, Andoh T, Lai AR, Lai E and Krishna G: Neuroprotective strategies in Parkinson's disease: protection against progressive nigral damage induced by free radicals. Neurotox Res 2000, 2:293-310.

1001. Christen Y: Oxidative stress and Alzheimer disease. American Journal of Clinical Nutrition 2000, 71:62 IS-629S.

1002. Floor E: Iron as a vulnerability factor in nigrostriatal degeneration in aging and Parkinson's disease. Cell Mol Biol (Noisy-le-grand) 2000, 46:709-20.

1003. Smith MA, Rottkamp CA, Nunomura A, Raina AK and Perry G: Oxidative stress in Alzheimer's disease. Biochim Biophys Acta 2000, I 502: 139-44

1004. Varadarajan S, Yatin S, Aksenova M and Butterfield DA: Alzheimer's amyloid beta-peptide-associated free radical oxidative stress and neurotoxicity. J Struct Biol 2000, I30:184-208.

1005. Berg D, Gerlach M, Youdim MBH, Double KL, Zecca L, Riederer P and Becker G: Brain iron pathways and their relevance to Parkinson's disease. I Neurochem 200I, 79:225-236.

1006. Blum D, Torch S, Lambeng N, Nissou MF, Benabid AL, Sadoul R and Verna JM: Molecular pathways involved in the neurotoxicity of 6-OHDA, dopamine and MPTP: contribution to the apoptotic theory in Parkinson's disease. Progress in Neurobiology 2001, 65:135-I72.

1007. Campbell A, Smith MA, Sayre LM, Bondy SC and Perry G: Mechanisms by which metals promote events connected to neurodegenerative diseases. Brain Research Bulletin 2001, 55: $125-132$

1008. Halliwell B: Role of free radicals in the neurodegenerative diseases - Therapeutic implications for antioxidant treatment. Drugs \& Aging 200I, I8:685-7|6.

1009. HaMai D, Bondy SC, Becaria A and Campbell A: The chemistry of transition metals in relation to their potential role in neurodegenerative processes. Curr Top Med Chem 2001, I:54I-5I.

1010. Rottkamp CA, Raina AK, Zhu XW, Gaier E, Bush Al, Atwood CS, Chevion M, Perry G and Smith MA: Redox-active iron mediates amyloid-beta toxicity. Free Rad Biol Med 200I, 30:447-450.

I0II. Sayre LM, Smith MA and Perry G: Chemistry and biochemistry of oxidative stress in neurodegenerative disease. Current Medicinal Chemistry 200I, 8:72I-738.

1012. Bharath S, Hsu M, Kaur D, Rajagopalan S and Andersen JK: Glutathione, iron and Parkinson's disease. Biochem Pharmacol 2002, 64: 1037-48.

1013. Bishop GM, Robinson SR, Liu Q, Perry G, Atwood CS and Smith MA: Iron: a pathological mediator of Alzheimer disease? Dev Neurosci 2002, 24:184-7.

1014. Giasson BI, Ischiropoulos H, Lee VM and Trojanowski JQ: The relationship between oxidative/nitrative stress and pathological inclusions in Alzheimer's and Parkinson's diseases. Free Radic Biol Med 2002, 32:1264-75.

1015. Gnjec A, Fonte JA, Atwood C and Martins RN: Transition metal chelator therapy - A potential treatment for Alzheimer's disease? Frontiers in Bioscience 2002, 7:DI016-DI023.

1016. Perry G, Sayre LM, Atwood CS, Castellani RJ, Cash AD, Rottkamp CA and Smith MA: The role of iron and copper in the aetiology of neurodegenerative disorders - Therapeutic implications. CNS Drugs 2002, 16:339-352.

1017. Rao AV and Balachandran B: Role of oxidative stress and antioxidants in neurodegenerative diseases. Nutritional Neuroscience 2002, 5:291-309.

I018. Burdo JR and Connor JR: Brain iron uptake and homeostatic mechanisms: An overview. Biometals 2003, 16:63-75.

1019. Bush Al: The metallobiology of Alzheimer's disease. Trends Neurosci 2003, 26:207-214

1020. Finefrock AE, Bush $\mathrm{Al}$ and Doraiswamy PM: Current status of metals as therapeutic targets in Alzheimer's disease. J Am Geriatr Soc 2003, 5 I: I| 43-8.

I02I. Ke Y and Qian ZM: Iron misregulation in the brain: a primary cause of neurodegenerative disorders. Lancet Neurol 2003 , 2:246-253.

1022. Barnham KJ, Masters CL and Bush Al: Neurodegenerative diseases and oxidative stress. Nat Rev Drug Discov 2004, 3:205-14.

1023. Bondy SC, Lahiri DK, Perreau VM, Sharman KZ, Campbell A Zhou J and Sharman EH: Retardation of brain aging by chronic treatment with melatonin. Ann N Y Acad Sci 2004, 1035:197-215.

1024. Casadesus G, Smith MA, Zhu X, Aliev G, Cash AD, Honda K, Petersen RB and Perry G: Alzheimer disease: evidence for a 
central pathogenic role of iron-mediated reactive oxygen species. J Alzheimers Dis 2004, 6:165-9.

1025. Doraiswamy PM and Finefrock AE: Metals in our minds: therapeutic implications for neurodegenerative disorders. Lancet Neurology 2004, 3:43 I-434.

1026. Gotz ME, Double K, Gerlach M, Youdim MBH and Riederer P: The relevance of iron in the pathogenesis of Parkinson's disease. Redox-Active Metals in Neurological Disorders, Annals of the New York Academy of Sciences 2004, I 0 I 2: I 93-208.

1027. Huang X, Moir RD, Tanzi RE, Bush Al and Rogers JT: Redoxactive metals, oxidative stress, and Alzheimer's disease pathology. Ann N Y Acad Sci 2004, I 01 2: 153-63.

1028. Kalivendi SV, Cunningham S, Kotamraju S, Joseph J, Hillard CJ and Kalyanaraman B: alpha-synuclein up-regulation and aggregation during MPP+-induced apoptosis in neuroblastoma cells - Intermediacy of transferrin receptor iron and hydrogen peroxide. Journal of Biological Chemistry 2004, 279: I5240-15247.

1029. Kaur D and Andersen J: Does cellular iron dysregulation play a causative role in Parkinson's disease? Ageing Res Rev 2004, 3:327-343

1030. Mattson MP: Metal-catalyzed disruption of membrane protein and lipid signaling in the pathogenesis of neurodegenerative disorders. Ann N Y Acad Sci 2004, I 0 I 2:37-50.

103 I. Mattson MP: Pathways towards and away from Alzheimer's disease. Nature 2004, 430:631-9.

1032. Milton NGN: Role of hydrogen peroxide in the aetiology of Alzheimer's disease - Implications for treatment. Drugs \& Aging 2004, 21 :8I-100.

1033. Moos T and Morgan EH: The metabolism of neuronal iron and its pathogenic role in neurological disease - Review. Ann NY Acad Sci 2004, I 0 I 2: 14-26.

1034. Poon HF, Calabrese V, Scapagnini G and Butterfield DA: Free radicals and brain aging. Clin Geriatr Med 2004, 20:329-59.

1035. Richardson DR: Novel chelators for central nervous system disorders that involve alterations in the metabolism of iron and other metal ions. Ann N Y Acad Sci 2004, I 0 I 2:326-4I.

1036. Warner DS, Sheng $\mathrm{H}$ and Batinić-Haberle I: Oxidants, antioxidants and the ischemic brain. J Exp Biol 2004, 207:322I-3I.

1037. Youdim MBH, Fridkin $M$ and Zheng $\mathrm{H}$ : Novel bifunctional drugs targeting monoamine oxidase inhibition and iron chelation as an approach to neuroprotection in Parkinson's disease and other neurodegenerative diseases. J Neural Transmission 2004, I I I:|455-|47|.

1038. Zecca L, Youdim MBH, Riederer P, Connor JR and Crichton RR: Iron, brain ageing and neurodegenerative disorders. Nat Rev Neurosci 2004, 5:863-873.

1039. Zhu X, Raina AK, Lee HG, Casadesus G, Smith MA and Perry G: Oxidative stress signalling in Alzheimer's disease. Brain Res 2004, 1000:32-9.

1040. Calabrese V, Lodi R, Tonon C, D'Agata V, Sapienza M, Scapagnini G, Mangiameli A, Pennisi G, Stella AMG and Butterfield DA: Oxidative stress, mitochondrial dysfunction and cellular stress response in Friedreich's ataxia. Journal of the Neurological Sciences 2005, 233: |45-162.

104I. Gaeta A and Hider RC: The crucial role of metal ions in neurodegeneration: the basis for a promising therapeutic strategy. Br J Pharmacol 2005, I 46: 1041-59.

1042. Hald A and Lotharius J: Oxidative stress and inflammation in Parkinson's disease: Is there a causal link? Exp Neurol 2005, 193:279-290.

1043. Honda K, Smith MA, Zhu X, Baus D, Merrick WC, Tartakoff AM, Hattier T, Harris PL, Siedlak SL, Fujioka H, Liu Q, Moreira PI, Miller FP, Nunomura A, Shimohama S and Perry G: Ribosomal RNA in Alzheimer disease is oxidized by bound redoxactive iron. J Biol Chem 2005, 280:20978-86.

1044. Ide-Ektessabi $A$ and Rabionet $M$ : The role of trace metallic elements in neurodegenerative disorders: quantitative analysis using XRF and XANES spectroscopy. Anal Sci 2005, 2 I:885-92.

1045. Liu G, Garrett MR, Men P, Zhu XW, Perry G and Smith MA: Nanoparticle and other metal chelation therapeutics in Alzheimer disease. Biochimica Et Biophysica Acta-Molecular Basis of Disease 2005, I741:246-252.

1046. Maynard CJ, Bush Al, Masters CL, Cappai R and Li QX: Metals and amyloid-beta in Alzheimer's disease. Int J Exp Pathol 2005, 86: $147-159$.

1047. Ong WY and Farooqui AA: Iron, neuroinflammation, and Alzheimer's disease. J Alzheimers Dis 2005, 8: I83-200.
1048. Adlard PA and Bush Al: Metals and Alzheimer's disease. J Alzheimers Dis 2006, I0:|45-163.

1049. Aracena P, Aguirre P, Munoz P and Nunez MT: Iron and glutathione at the crossroad of redox metabolism in neurons. Biol Res 2006, 39:|57-65.

1050. Berg D and Hochstrasser H: Iron metabolism in parkinsonian syndromes. Movement Disorders 2006, 21:I299-1310.

I05I. Collingwood J and Dobson J: Mapping and characterization of iron compounds in Alzheimer's tissue. J Alzheimers Dis 2006, 10:215-222.

1052. Fasano M, Bergamasco B and Lopiano L: Modifications of the iron-neuromelanin system in Parkinson's disease. J Neurochem 2006, 96:909-916.

1053. Gaggelli E, Kozlowski H, Valensin D and Valensin G: Copper homeostasis and neurodegenerative disorders (Alzheimer's, prion, and Parkinson's diseases and amyotrophic lateral sclerosis). Chem Rev 2006, 106:1995-2044.

1054. Gerlach M, Double KL, Youdim MBH and Riederer P: Potential sources of increased iron in the substantia nigra of parkinsonian patients. J Neural Transmission - Suppl 2006, $133-142$.

1055. Halliwell B: Oxidative stress and neurodegeneration: where are we now? Journal of Neurochemistry 2006, 97:1634-1658.

1056. Huber A, Stuchbury G, Burkle A, Burnell J and Munch G: Neuroprotective therapies for Alzheimer's disease. Curr Pharmaceut Des 2006, I2:705-717.

1057. Liu GJ, Huang WD, Moir RD, Vanderburg CR, Lai B, Peng ZC, Tanzi RE, Rogers JT and Huang XD: Metal exposure and Alzheimer's pathogenesis. J Struct Biol 2006, I55:45-5 I.

1058. Lee DW, Andersen JK and Kaur D: Iron dysregulation and neurodegeneration - The molecular connection. Molecular Interventions 2006, 6:89-97.

1059. Mancuso M, Coppede F, Migliore L, Siciliano G and Murri L: Mitochondrial dysfunction, oxidative stress and neurodegeneration. Journal of Alzheimers Disease 2006, 10:59-73.

1060. Mosley RL, Benner EJ, Kadiu I, Thomas M, Boska MD, Hasan K, Laurie $C$ and Gendelman HE: Neuroinflammation, oxidative stress, and the pathogenesis of Parkinson's disease. Clinical Neuroscience Research 2006, 6:26I-28I.

106I. Quintana C, Bellefqih S, Laval JY, Guerquin-Kern JL, Wu TD, Avila J, Ferrer I, Arranz R and Patino C: Study of the localization of iron, ferritin, and hemosiderin in Alzheimer's disease hippocampus by analytical microscopy at the subcellular level. J Struct Biol 2006, I 53:42-54.

1062. Whitnall $M$ and Richardson DR: Iron: a new target for pharmacological intervention in neurodegenerative diseases. Semin Pediatr Neurol 2006, 13:186-97.

1063. Zhang HY: Same causes, same cures. Biochem Biophys Res Commun 2006, 351:578-581.

1064. Bartzokis G, Tishler TA, Lu PH, Villablanca P, Altshuler LL, Carter M, Huang D, Edwards $\mathrm{N}$ and Mintz J: Brain ferritin iron may influence age- and gender-related risks of neurodegeneration. Neurobiol Aging 2007, 28:4 I4-23

1065. Bondy SC and Sharman EH: Melatonin and the aging brain. Neurochem Int 2007, 50:57I-80.

1066. Cass WA, Grondin R, Andersen AH, Zhang Z, Hardy PA, HusseyAndersen LK, Rayens WS, Gerhardt GA and Gash DM: Iron accumulation in the striatum predicts aging-related decline in motor function in rhesus monkeys. Neurobiol Aging 2007, 28:258-7l.

1067. Castellani RJ, Moreira PI, Liu G, Dobson J, Perry G, Smith MA and Zhu X: Iron: the Redox-active center of oxidative stress in Alzheimer disease. Neurochem Res 2007, 32:1640-5.

1068. Crouch PJ, White AR and Bush Al: The modulation of metal bio-availability as a therapeutic strategy for the treatment of Alzheimer's disease. FEBS J 2007, 274:3775-83.

1069. Donnelly PS, Xiao Z and Wedd AG: Copper and Alzheimer's disease. Curr Opin Chem Biol 2007, I I:I28-33.

1070. Gaasch JA, Lockman PR, Geldenhuys WJ, Allen DD and Schyf Van der Cl: Brain iron toxicity: differential responses of astrocytes, neurons, and endothelial cells. Neurochem Res 2007 32: I 196-208.

107I. Ke Y and Qian ZM: Brain iron metabolism: neurobiology and neurochemistry. Prog Neurobiol 2007, 83:|49-73.

1072. Mancuso C, Scapagnini G, Curro D, Stella AMG, De Marco C, Butterfield DA and Calabrese V: Mitochondrial dysfunction, free radical generation and cellular stress response in neurodegenerative disorders. Frontiers in Bioscience 2007, I2: I 107-II23. 
1073. Mandel S, Amit T, Bar-Am O and Youdim MB: Iron dysregulation in Alzheimer's disease: multimodal brain permeable iron chelating drugs, possessing neuroprotective-neurorescue and amyloid precursor protein-processing regulatory activities as therapeutic agents. Prog Neurobiol 2007, 82:348-60

1074. Molina-Holgado F, Hider RC, Gaeta A, Williams R and Francis P: Metals ions and neurodegeneration. Biometals 2007, 20:639-54.

1075. Petersen RB, Nunomura A, Lee HG, Casadesus G, Perry G, Smith MA and Zhu X: Signal transduction cascades associated with oxidative stress in Alzheimer's disease. J Alzheimers Dis 2007, I I:|43-52.

1076. Reynolds A, Laurie C, Mosley RL and Gendelman HE: Oxidative stress and the pathogenesis of neurodegenerative disorders. Int Rev Neurobiol 2007, 82:297-325.

1077. Singh C, Ahmad I and Kumar A: Pesticides and metals induced Parkinson's disease: involvement of free radicals and oxidative stress. Cell Mol Biol (Noisy-le-grand) 2007, 53:19-28.

1078. Stankiewicz J, Panter SS, Neema M, Arora A, Batt CE and Bakshi R: Iron in chronic brain disorders: imaging and neurotherapeutic implications. Neurotherapeutics 2007, 4:37|-86.

1079. Zhu X, Su B, Wang X, Smith MA and Perry G: Causes of oxidative stress in Alzheimer disease. Cell Mol Life Sci 2007, 64:2202-10.

1080. Amit T, Avramovich-Tirosh Y, Youdim MB and Mandel S: Targeting multiple Alzheimer's disease etiologies with multimodal neuroprotective and neurorestorative iron chelators. FASEB J 2008, 22:1296-305.

$108 \mathrm{I}$. Bush Al and Curtain CC: Twenty years of metallo-neurobiology: where to now? Eur Biophys J 2008, 37:24 I-5.

1082. Drechsel DA and Patel M: Role of reactive oxygen species in the neurotoxicity of environmental agents implicated in Parkinson's disease. Free Radic Biol Med 2008, 44:I873-86.

1083. Pankhurst Q, Hautot D, Khan N and Dobson J: Increased levels of magnetic iron compounds in Alzheimer's disease. J Alzheimers Dis 2008, I 3:49-52.

1084. Rogers JT, Bush Al, Cho HH, Smith DH, Thomson AM, Friedlich AL, Lahiri DK, Leedman PJ, Huang $X$ and Cahill CM: Iron and the translation of the amyloid precursor protein (APP) and ferritin mRNAs: riboregulation against neural oxidative damage in Alzheimer's disease. Biochem Soc Trans 2008, 36: I282-7.

1085. Silvestri $L$ and Camaschella C: A potential pathogenetic role of iron in Alzheimer's Disease. J Cell Mol Med 2008, I 2: 1548-1550.

1086. Snyder AM and Connor JR: Iron, the substantia nigra and related neurological disorders. Biochim Biophys Acta 2008 in press.

1087. Szczerbowska-Boruchowska M: X-ray fluorescence spectrometry, an analytical tool in neurochemical research. $X-R a y$ Spectrometry 2008, 37:2I-3I.

1088. Sayre LM, Perry G, Harris PLR, Liu YH, Schubert KA and Smith MA: In situ oxidative catalysis by neurofibrillary tangles and senile plaques in Alzheimer's disease: A central role for bound transition metals. Journal of Neurochemistry 2000, 74:270-279.

1089. Vélez-Pardo C, Del Río MJ, Verschueren H, Ebinger $G$ and Vauquelin G: Dopamine and iron induce apoptosis in PCI2 cells. Pharmacol Toxicol 1997, 80:76-84.

1090. Kostoff RN and Briggs MB: Literature-Related Discovery (LRD): potential treatments for Parkinson's disease. Technol. Forecast. Soc. Change 2007.

1091. LeVine SM and Chakrabarty A: The role of iron in the pathogenesis of experimental allergic encephalomyelitis and multiple sclerosis. Redox-Active Metals in Neurological Disorders, Annals of the New York Academy of Sciences 2004, | 0 | 2:252-266.

1092. Kotze MJ, de Villiers JNP, Rooney RN, Grobbelaar JJ, Mansvelt EPG, Bouwens CSH, Carr J, Stander I and du Plessis L: Analysis of the NRAMPI gene implicated in iron transport: Association with multiple sclerosis and age effects. Blood Cells Molecules and Diseases 2001, 27:44-53.

1093. LeVine SM, Lynch SG, Ou CN, Wulser MJ, Tam E and Boo N: Ferritin, transferrin and iron concentrations in the cerebrospinal fluid of multiple sclerosis patients. Brain Research |999, 82 I:5I I-5 I 5.

1094. Valberg LS, Flanagan PR, Kertesz A and Ebers GC: Abnormalities in Iron-Metabolism in Multiple-Sclerosis. Canadian Journal of Neurological Sciences 1989, 16:184-186.
1095. Adams CWM: Perivascular Iron Deposition and Other Vascular Damage in Multiple-Sclerosis. Journal of Neurology Neurosurgery and Psychiatry 1988, 5 I:260-265.

1096. Drayer B, Burger P, Hurwitz B, Dawson D and Cain J: Reduced Signal Intensity on Mr Images of Thalamus and Putamen in Multiple-Sclerosis - Increased Iron Content. American Journal of Neuroradiology 1987, 8:413-419.

1097. Craelius W, Migdal MW, Luessenhop CP, Sugar A and Mihalakis I: Iron Deposits Surrounding Multiple-Sclerosis Plaques. Archives of Pathology \& Laboratory Medicine 1982, I06:397-399.

1098. Ge Y, Jensen JH, Lu H, Helpern JA, Miles L, Inglese M, Babb JS, Herbert $J$ and Grossman RI: Quantitative assessment of iron accumulation in the deep gray matter of multiple sclerosis by magnetic field correlation imaging. AJNR Am J Neuroradiol 2007, 28:1639-44.

1099. Kim NH, Choi JK, Jeong BH, Kim JI, Kwon MS, Carp RI and Kim YS: Effect of transition metals ( $\mathrm{Mn}, \mathrm{Cu}, \mathrm{Fe}$ ) and deoxycholic acid (DA) on the conversion of PrPC to PrPres. FASEB J 2005, I9:783-5.

1 100. Basu S, Mohan ML, Luo X, Kundu B, Kong $Q$ and Singh $N$ : Modulation of proteinase K-resistant prion protein in cells and infectious brain homogenate by redox iron: implications for prion replication and disease pathogenesis. Mol Biol Cell 2007, I8:3302-I2.

IIOI. Lill R and Muhlenhoff U: Maturation of iron-sulfur proteins in eukaryotes: mechanisms, connected processes, and diseases. Annu Rev Biochem 2008, 77:669-700.

I 102. Cavadini P, O'Neill HA, Benada $O$ and Isaya G: Assembly and iron-binding properties of human frataxin, the protein deficient in Friedreich ataxia. Hum Mol Genet 2002, I I:2 17-27.

I I03. Napier I, Ponka P and Richardson DR: Iron trafficking in the mitochondrion: novel pathways revealed by disease. Blood 2005, I05:1867-74

I 104. O'Neill HA, Gakh O, Park S, Cui J, Mooney SM, Sampson M, Ferreira GC and Isaya G: Assembly of human frataxin is a mechanism for detoxifying redox-active iron. Biochemistry 2005, 44:537-45.

I I05. Gakh O, Park S, Liu G, Macomber L, Imlay JA, Ferreira GC and Isaya G: Mitochondrial iron detoxification is a primary function of frataxin that limits oxidative damage and preserves cell Iongevity. Human Molecular Genetics 2006, I5:467-479.

I 106. Napoli E, Taroni F and Cortopassi GA: Frataxin, iron-sulfur clusters, heme, ROS, and aging. Antioxid Redox Signal 2006, 8:506-16.

I I07. Babady NE, Carelle N, Wells RD, Rouault TA, Hirano M, Lynch DR, Delatycki MB, Wilson RB, Isaya G and Puccio H: Advancements in the pathophysiology of Friedreich's Ataxia and new prospects for treatments. Mol Genet Metab 2007, 92:23-35.

1 108. Lu C and Cortopassi G: Frataxin knockdown causes loss of cytoplasmic iron-sulfur cluster functions, redox alterations and induction of heme transcripts. Arch Biochem Biophys 2007. 457: I ||-22.

I 109. Zanella I, Derosas M, Corrado M, Cocco E, Cavadini P, Biasiotto G, Poli M, Verardi R and Arosio P: The effects of frataxin silencing in HeLa cells are rescued by the expression of human mitochondrial ferritin. Biochim Biophys Acta 2008, I 782:90-8.

I I I0. Anderson PR, Kirby K, Hilliker AJ and Phillips JP: RNAi-mediated suppression of the mitochondrial iron chaperone, frataxin, in Drosophila. Hum Mol Genet 2005, I 4:3397-405.

IIII. Jo WJ, Loguinov A, Chang M, Wintz H, Nislow C, Arkin AP, Giaever $G$ and Vulpe $C D$ : Identification of genes involved in the toxic response of Saccharomyces cerevisiae against iron and copper overload by parallel analysis of deletion mutants. Toxicol Sci 2008, I0I:|40-5|.

III2. Gibson TJ, Koonin EV, Musco G, Pastore A and Bork P: Friedreich's ataxia protein: phylogenetic evidence for mitochondrial dysfunction. Trends Neurosci 1996, 19:465-8.

III3. Koutnikova H, Campuzano V, Foury F, Dolle P, Cazzalini $O$ and Koenig M: Studies of human, mouse and yeast homologues indicate a mitochondrial function for frataxin. Nat Genet 1997, 16:345-51.

I I 14. Foury F: Human genetic diseases: A cross-talk between man and yeast. Gene 1997, 195:I-10.

III5. Foury F and Cazzalini O: Deletion of the yeast homologue of the human gene associated with Friedreich's ataxia elicits iron accumulation in mitochondria. FEBS Lett 1997 , 4 I I:373-377. 
III6. Cavadini P, Gellera C, Patel PI and Isaya G: Human frataxin maintains mitochondrial iron homeostasis in Saccharomyces cerevisiae. Hum Mol Genet 2000, 9:2523-30.

III7. Becker E and Richardson DR: Frataxin: its role in iron metabolism and the pathogenesis of Friedreich's ataxia. Int J Biochem Cell Biol 200I, 33:I-10.

III8. Foury F and Talibi D: Mitochondrial control of iron homeostasis. A genome wide analysis of gene expression in a yeast frataxin-deficient strain. J Biol Chem 200I, 276:7762-8.

III9. De Freitas J, Wintz H, Kim JH, Poynton H, Fox T and Vulpe C: Yeast, a model organism for iron and copper metabolism studies. Biometals 2003, I 6: 185-197.

I120. González-Cabo P, Vázquez-Manrique RP, García-Gimeno MA, Sanz $P$ and Palau F: Frataxin interacts functionally with mitochondrial electron transport chain proteins. Hum $\mathrm{Mol}$ Genet 2005, |4:2091-8.

II2I. Irazusta V, Cabiscol E, Reverter-Branchat G, Ros J and Tamarit J: Manganese is the link between frataxin and iron-sulfur deficiency in the yeast model of Friedreich ataxia. J Biol Chem 2006, 28 I: | 2227-32.

I 122. Vázquez-Manrique RP, González-Cabo P, Ros S, Aziz H, Baylis HA and Palau F: Reduction of Caenorhabditis elegans frataxin increases sensitivity to oxidative stress, reduces lifespan, and causes lethality in a mitochondrial complex II mutant. Faseb J 2006, 20:172-4.

I 23. Irazusta V, Moreno-Cermeno A, Cabiscol E, Ros J and Tamarit ]: Major targets of iron-induced protein oxidative damage in frataxin-deficient yeasts are magnesium-binding proteins. Free Radic Biol Med 2008.

I 124. Campuzano V, Montermini L, Molto MD, Pianese L, Cossee M, Cavalcanti F, Monros E, Rodius F, Duclos F, Monticelli A, Zara F, Canizares ], Koutnikova H, Bidichandani SI, Gellera C, Brice A, Trouillas P, De Michele G, Filla A, De Frutos R, Palau F, Patel PI, Di Donato S, Mandel JL, Cocozza S, Koenig $M$ and Pandolfo $M$ : Friedreich's ataxia: autosomal recessive disease caused by an intronic GAA triplet repeat expansion. Science 1996, 27|:|423-7.

I I25. Palau F: Friedreich's ataxia and frataxin: molecular genetics, evolution and pathogenesis (Review). Int J Mol Med 200I, 7:581-9.

I 26. Patel PI and Isaya G: Friedreich ataxia: from GAA tripletrepeat expansion to frataxin deficiency. Am J Hum Genet 200 I, 69:15-24.

I I27. Wilson RB: Frataxin and frataxin deficiency in Friedreich's ataxia. J Neurol Sci 2003, 207:103-5.

II28. Michael S, Petrocine SV, Qian J, Lamarche JB, Knutson MD, Garrick $M D$ and Koeppen $A H$ : Iron and iron-responsive proteins in the cardiomyopathy of Friedreich's ataxia. Cerebellum 2006, 5:257-67.

I 129. Rötig A, Sidi D, Munnich A and Rustin P: Molecular insights into Friedreich's ataxia and antioxidant-based therapies. Trends Mol Med 2002, 8:22I-4.

I 130. Boddaert N, Le Quan Sang KH, Rotig A, Leroy-Willig A, Gallet S Brunelle F, Sidi D, Thalabard JC, Munnich A and Cabantchik ZI: Selective iron chelation in Friedreich ataxia: biologic and clinical implications. Blood 2007, I 1 0:40 I-8

I|3I. Sohn YS, Breuer W, Munnich A and Cabantchik ZI: Redistribution of accumulated cell iron: a modality of chelation with therapeutic implications. Blood 2008, I I I:I690-9.

I132. Besarab A, Bolton WK, Browne JK, Egrie JC, Nissenson AR, Okamoto DM, Schwab SJ and Goodkin DA: The effects of normal as compared with low hematocrit values in patients with cardiac disease who are receiving hemodialysis and epoetin. N Engl J Med 1998, 339:584-90.

I133. Campuzano V, Montermini L, Lutz Y, Cova L, Hindelang C, Jiralerspong S, Trottier Y, Kish SJ, Faucheux B, Trouillas P, Authier FJ, Durr A, Mandel JL, Vescovi A, Pandolfo $M$ and Koenig $M$ : Frataxin is reduced in Friedreich ataxia patients and is associated with mitochondrial membranes. Hum $\mathrm{Mol}$ Genet 1997, 6: I77|-80.

I 134. Wong A, Yang J, Cavadini P, Gellera C, Lonnerdal B, Taroni F and Cortopassi G: The Friedreich's ataxia mutation confers cellular sensitivity to oxidant stress which is rescued by chelators of iron and calcium and inhibitors of apoptosis. Hum Mol Genet 1999, 8:425-30.

I 135. Park S, Gakh O, Mooney SM and Isaya G: The ferroxidase activity of yeast frataxin. J Biol Chem 2002, 277:38589-95.

I 136. Sturm B, Bistrich U, Schranzhofer M, Sarsero IP, Rauen U, ScheiberMojdehkar B, de Groot H, loannou P and Petrat F: Friedreich's ataxia, no changes in mitochondrial labile iron in human lymphoblasts and fibroblasts: a decrease in antioxidative capacity? J Biol Chem 2005, 280:670I-8.

I137. Anderson PR, Kirby K, Orr WC, Hilliker AJ and Phillips JP: Hydrogen peroxide scavenging rescues frataxin deficiency in a Drosophila model of Friedreich's ataxia. Proc Natl Acad Sci USA 2008, 105:611-6.

I 138. Wilson RB and Roof DM: Respiratory deficiency due to loss of mitochondrial DNA in yeast lacking the frataxin homologue. Nat Genet 1997, 16:352-7.

I139. Seznec H, Simon D, Bouton C, Reutenauer L, Hertzog A, Golik P, Procaccio V, Patel M, Drapier JC, Koenig $M$ and Puccio H: Friedreich ataxia: the oxidative stress paradox. Hum Mol Genet 2005, 14:463-74.

I 140. Visapää I, Fellman V, Vesa J, Dasvarma A, Hutton JL, Kumar V, Payne GS, Makarow M, Van Coster R, Taylor RW, Turnbull DM, Suomalainen A and Peltonen L: GRACILE syndrome, a lethal metabolic disorder with iron overload, is caused by a point mutation in BCSIL. Am J Hum Genet 2002, 71:863-76.

I|4I. Fellman V, Lemmela S, Sajantila A, Pihko H and Jarvela I: Screening of $B C S I L$ mutations in severe neonatal disorders suspicious for mitochondrial cause. J Hum Genet 2008, 53:554-8.

1|42. McGeer PL and McGeer EG: Inflammatory processes in amyotrophic lateral sclerosis. Muscle Nerve 2002, 26:459-70.

I 143. Garber K: The elusive ALS genes. Science 2008, 3 1 9:20.

I|44. Rosen DR, Siddique T, Patterson D, Figlewicz DA, Sapp P, Hentati A, Donaldson D, Goto J, O'Regan JP and Deng HX, et al: Mutations in $\mathrm{Cu} / \mathrm{Zn}$ superoxide dismutase gene are associated with familial amyotrophic lateral sclerosis. Nature 1993, 362:59-62.

I 145. Kruman II, Pedersen WA, Springer JE and Mattson MP: ALS-linked $\mathrm{Cu} / \mathrm{Zn}$-SOD mutation increases vulnerability of motor neurons to excitotoxicity by a mechanism involving increased oxidative stress and perturbed calcium homeostasis. Exp Neurol 1999, 160:28-39.

II46. Bruijn LI, Miller TM and Cleveland DW: Unraveling the mechanisms involved in motor neuron degeneration in ALS. Annu Rev Neurosci 2004, 27:723-49.

I I47. Kasarskis EJ, Tandon L, Lovell MA and Ehmann WD: Aluminum, calcium, and iron in the spinal cord of patients with sporadic amyotrophic lateral sclerosis using laser microprobe mass spectroscopy: a preliminary study. J Neurol Sci 1995, I30:203-8

I 48. Carri MT, Ferri A, Cozzolino M, Calabrese L and Rotilio G: Neurodegeneration in amyotrophic lateral sclerosis: the role of oxidative stress and altered homeostasis of metals. Brain Res Bull 2003, 61:365-74.

I|49. Carri MT, Grignaschi G and Bendotti C: Targets in ALS: designing multidrug therapies. Trends Pharmacol Sci 2006, 27:267-73.

II50. Hughes KA and Reynolds RM: Evolutionary and mechanistic theories of aging. Annual Review of Entomology 2005, 50:42 I-445.

II5I. Zacharski LR, Ornstein DL, Woloshin S and Schwartz LM: Association of age, sex, and race with body iron stores in adults: analysis of NHANES III data. Am Heart J 2000, 140:98-104.

I I52. Ritchie RF, Palomaki GE, Neveux LM, Navolotskaia O, Ledue TB and Craig WY: Reference distributions for serum iron and transferrin saturation: a comparison of a large cohort to the world's literature. J Clin Lab Anal 2002, 16:246-52.

I 153. Ritchie RF, Palomaki GE, Neveux LM, Navolotskaia O, Ledue TB and Craig WY: Reference distributions for serum iron and transferrin saturation: a practical, simple, and clinically relevant approach in a large cohort. J Clin Lab Anal 2002, 16:237-45

I I54. Liu JM, Hankinson SE, Stampfer MJ, Rifai N, Willett WC and Ma J: Body iron stores and their determinants in healthy postmenopausal US women. Am J Clin Nutr 2003, 78:1 160-7.

1155. Doulias PT, Vlachou C, Boudouri C, Kanavaros P, Siamopoulos KC and Galaris D: Flow cytometric estimation of 'labile iron pool' in human white blood cells reveals a positive association with ageing. Free Radic Res 2008, 42:253-9.

I 156. Fleming DJ, Tucker KL, Jacques PF, Dallal GE, Wilson PW and Wood RJ: Dietary factors associated with the risk of high iron stores in the elderly Framingham Heart Study cohort. Am J Clin Nutr 2002, 76: I375-84.

I 157. Beard J: Dietary iron intakes and elevated iron stores in the elderly: is it time to abandon the set-point hypothesis of regulation of iron absorption? Am / Clin Nutr 2002, 76: I I89-90.

I I58. Cade JE, Moreton JA, O'Hara B, Greenwood DC, Moor J, Burley VJ, Kukalizch K, Bishop DT and Worwood M: Diet and genetic 
factors associated with iron status in middle-aged women. Am I Clin Nutr 2005, 82:8I3-20.

II59. Guralnik JM, Eisenstaedt RS, Ferrucci L, Klein HG and Woodman RC: Prevalence of anemia in persons 65 years and older in the United States: evidence for a high rate of unexplained anemia. Blood 2004, 104:2263-8.

I 160. Price EA: Aging and erythropoiesis: current state of knowledge. Blood Cells Mol Dis 2008, 4I:I58-65.

II6I. Sharman EH, Bondy SC, Sharman KG, Lahiri D, Cotman CW and Perreau VM: Effects of melatonin and age on gene expression in mouse CNS using microarray analysis. Neurochemistry International 2007, 50:336-344.

I I62. Kirkwood TBL: Evolution of Aging. Nature 1977, 270:30I-304.

I 163. Beckman $K B$ and Ames $B N$ : The free radical theory of aging matures. Physiol Rev |998, 78:547-8I.

I I64. Finkel $\mathrm{T}$ and Holbrook NJ: Oxidants, oxidative stress and the biology of ageing. Nature 2000, 408:239-47.

I I65. Sohal RS, Mockett RJ and Orr WC: Current issues concerning the role of oxidative stress in aging: a perspective. Results Probl Cell Differ 2000, 29:45-66.

I166. Cutler RG and Rodriguez $\mathrm{H}$ : Critical reviews of oxidative stress and aging: advances in basic science, diagnostics and intervention. World Scientific, Singapore 2002.

II67. Herndon LA, Schmeissner PJ, Dudaronek JM, Brown PA, Listner KM, Sakano Y, Paupard MC, Hall DH and Driscoll M Stochastic and genetic factors influence tissue-specific decline in ageing C. elegans. Nature 2002, 419:808-14.

I I68. Sohal RS, Mockett RJ and Orr WC: Mechanisms of aging: an appraisal of the oxidative stress hypothesis. Free Radic Biol Med 2002, 33:575-86.

I169. Partridge L and Gems D: Mechanisms of ageing: public or private? Nat Rev Genet 2002, 3:165-75.

I I70. Hekimi S and Guarente L: Genetics and the specificity of the aging process. Science 2003, 299:|35|-4

II7I. Krabbe KS, Pedersen $M$ and Bruunsgaard $\mathrm{H}$ : Inflammatory mediators in the elderly. Exp Gerontol 2004, 39:687-99.

I 172. Longo VD and Finch CE: Evolutionary medicine: from dwarf model systems to healthy centenarians? Science 2003 , 299: 1342-6.

1 173. McGeer PL and McGeer EG: Inflammation and the degenerative diseases of aging. Ann N Y Acad Sci 2004, 1035: 104-16.

I I74. Afanas'ev IB: Free radical mechanisms of aging processes under physiological conditions. Biogerontology 2005, 6:283-90.

I I75. Kirkwood TB: Understanding the odd science of aging. Cell 2005, I 20:437-47.

1176. Tuppo EE and Arias HR: The role of inflammation in Alzheimer's disease. Int | Biochem Cell Biol 2005, 37:289-305.

I I77. Partridge L and Gems D: Beyond the evolutionary theory of ageing, from functional genomics to evo-gero. Trends Ecol Evol 2006, 21:334-40.

1 178. Salvioli S, Capri M, Valensin S, Tieri P, Monti D, Ottaviani E and Franceschi C: Inflamm-aging, cytokines and aging: state of the art, new hypotheses on the role of mitochondria and new perspectives from systems biology. Curr Pharm Des 2006, |2:3|6|-7|.

I179. Franco OH, Kirkwood TB, Powell JR, Catt M, Goodwin J, Ordovas JM and Ouderaa van der F: Ten commandments for the future of ageing research in the UK: a vision for action. BMC Geriatr 2007, 7:10.

I 180. Kirkwood TB: A systematic look at an old problem. Nature 2008, 45I:644-7.

II8I. Harman D: Aging: a theory based on free radical and radiation chemistry. J Gerontol 1956, I I:298-300.

I 182. Ames BN, Shigenaga MK and Hagen TM: Oxidants, antioxidants, and the degenerative diseases of aging. Proc Natl Acad Sci USA 1993, 90:7915-22.

I 83. Martin GM, Austad SN and Johnson TE: Genetic analysis of ageing: role of oxidative damage and environmental stresses. Nat Genet 1996, 13:25-34.

I 184. Ishii N, Fujii M, Hartman PS, Tsuda M, Yasuda K, Senoo-Matsuda N, Yanase $S$, Ayusawa $D$ and Suzuki K: A mutation in succinate dehydrogenase cytochrome $b$ causes oxidative stress and ageing in nematodes. Nature 1998, 394:694-7.

I 185. Fukagawa NK: Aging: is oxidative stress a marker or is it causal? Proc Soc Exp Biol Med 1999, 222:293-8.

I 186. Johnson FB, Sinclair DA and Guarente L: Molecular biology of aging. Cell 1999, 96:291-302.

I 187. Melov S, Ravenscroft ], Malik S, Gill MS, Walker DW, Clayton PE, Wallace DC, Malfroy B, Doctrow SR and Lithgow GJ: Extension of life-span with superoxide dismutase/catalase mimetics. Science 2000, 289: I 567-9.

I I88. Hamilton ML, Van Remmen H, Drake JA, Yang H, Guo ZM, Kewitt K, Walter CA and Richardson A: Does oxidative damage to DNA increase with age? Proc Natl Acad Sci USA 200I, 98: 10469-74.

I 189. von Zglinicki T, Bürkle A and Kirkwood TBL: Stress, DNA damage and ageing - an integrative approach. Exp Gerontol 2001, 36:1049-1062.

I190. Barja G: Rate of generation of oxidative stress-related damage and animal longevity. Free Radical Biology and Medicine 2002, 33: 1167-1172.

I।9।. Bonilla E, Medina-Leendertz S and Diaz S: Extension of life span and stress resistance of Drosophila melanogaster by longterm supplementation with melatonin. Exp Gerontol 2002, 37:629-38.

1192. Golden TR, Hinerfeld DA and Melov S: Oxidative stress and aging: beyond correlation. Aging Cell 2002, I: I I 7-23.

I 193. Hagen TM: Oxidative stress, redox imbalance, and the aging process. Antioxidants \& Redox Signaling 2003, 5:503-506.

I 194. Karanjawala ZE, Murphy N, Hinton DR, Hsieh CL and Lieber MR: Oxygen metabolism causes chromosome breaks and is associated with the neuronal apoptosis observed in DNA double-strand break repair mutants. Curr Biol 2002, 1 2:397-402

II95. Melov S: Animal models of oxidative stress, aging, and therapeutic antioxidant interventions. Int J Biochem Cell Biol 2002, 34:1395-400.

I 196. Barros MH, Bandy B, Tahara EB and Kowaltowski AJ: Higher respiratory activity decreases mitochondrial reactive oxygen release and increases life span in Saccharomyces cerevisiae. Journal of Biological Chemistry 2004, 279:49883-49888.

I 197. Gredilla R and Barja G: Minireview: the role of oxidative stress in relation to caloric restriction and Iongevity. Endocrinology 2005, | 46:37|3-7.

I 198. Blagosklonny MV: Aging and immortality: quasi-programmed senescence and its pharmacologic inhibition. Cell Cycle 2006, 5:2087-102.

I 199. Chung HY, Sung B, Jung KJ, Zou Y and Yu BP: The molecular inflammatory process in aging. Antioxid Redox Signal 2006, 8:572-8I.

1200. Sedensky MM and Morgan PG: Mitochondrial respiration and reactive oxygen species in C. elegans. Exp Gerontol 2006, 4I:957-67.

I20I. Terman A and Brunk UT: Oxidative stress, accumulation of biological 'garbage', and aging. Antioxidants \& Redox Signaling 2006, 8: 197-204.

1202. Yu BP and Chung HY: Adaptive mechanisms to oxidative stress during aging. Mechanisms of Ageing and Development 2006, I 27:436-443.

1203. Chakravarti B and Chakravarti DN: Oxidative modification of proteins: Age-related changes. Gerontology 2007, 53:। 28-। 39.

1204. Kim SK: Common aging pathways in worms, flies, mice and humans. J Exp Biol 2007, 2 I 0:1607-12.

1205. Kregel KC and Zhang HJ: An integrated view of oxidative stress in aging: basic mechanisms, functional effects, and pathological considerations. Am I Physiol Regul Integr Comp Physiol 2007, 292:RI8-36.

1206. Nijnik A, Woodbine L, Marchetti C, Dawson S, Lambe T, Liu C, Rodrigues NP, Crockford TL, Cabuy E, Vindigni A, Enver T, Bell Jl, Slijepcevic P, Goodnow CC, Jeggo PA and Cornall RJ: DNA repair is limiting for haematopoietic stem cells during ageing. Nature 2007, 447:686-90.

1207. Sohal RS and Weindruch R: Oxidative stress, caloric restriction, and aging. Science 1996, 273:59-63.

1208. Pardon MC: Stress and ageing interactions: a paradox in the context of shared etiological and physiopathological processes. Brain Res Rev 2007, 54:25 I-73.

1209. Killilea DW, Atamna H, Liao C and Ames BN: Iron accumulation during cellular senescence in human fibroblasts in vitro. Antioxid Redox Signal 2003, 5:507-16.

1210. Hamerman D: Toward an understanding of frailty. Ann Int Med 1999, I30:945-950.

I2II. Docherty JR: Cardiovascular responses in aging - a review. Pharmacol Rev 1990, 42:103-125

1212. Dröge $W$ and Schipper HM: Oxidative stress and aberrant signaling in aging and cognitive decline. Aging Cell 2007, 6:361-370. 
1213. Massie HR, Aiello VR and Banziger V: Iron accumulation and lipid peroxidation in aging $\mathbf{C} 57 \mathbf{b l} / \mathbf{6 j}$ mice. Exp Gerontol I983, I 8:277-285.

1214. Ershler WB: Biological interactions of aging and anemia: $\mathbf{A}$ focus on cytokines. J Amer Geriatr Soc 2003, 51:SI8-S2I.

1215. Massie HR, Aiello VR and Williams TR: Inhibition of iron absorption prolongs the lifespan of Drosophila. Mech Ageing Dev 1993, 67:227-237.

1216. Rattan SIS: Aging, anti-aging, and hormesis. Mechanisms of Ageing and Development 2004, 125:285-289.

1217. Kenyon C: The plasticity of aging: insights from long-lived mutants. Cell 2005, I20:449-60.

1218. Lee CK, Klopp RG, Weindruch R and Prolla TA: Gene expression profile of aging and its retardation by caloric restriction. Science 1999, 285: 1390-3.

I219. Bordone L and Guarente L: Calorie restriction, SIRTI and metabolism: understanding longevity. Nat Rev Mol Cell Biol 2005, 6:298-305.

1220. Taguchi A, Wartschow LM and White MF: Brain IRS2 signaling coordinates life span and nutrient homeostasis. Science 2007 , 3 1 7:369-72.

I22I. Hulbert AJ, Pamplona R, Buffenstein R and Buttemer WA: Life and death: metabolic rate, membrane composition, and life span of animals. Physiol Rev 2007, 87: II75-2।3.

1222. Kennedy BK, Steffen KK and Kaeberlein M: Ruminations on dietary restriction and aging. Cell Mol Life Sci 2007, 64:I323-8.

1223. Mair W and Dillin A: Aging and survival: the genetics of life span extension by dietary restriction. Annu Rev Biochem 2008 77:727-54.

1224. Piper MD and Partridge L: Dietary restriction in Drosophila: delayed aging or experimental artefact? PLOS Genet 2007, 3: e57.

1225. Weindruch R and Sohal RS: Caloric intake and aging. New England Journal of Medicine 1997, 337:986-994.

1226. Lass A, Sohal BH, Weindruch R, Forster MJ and Sohal RS: Caloric restriction prevents age-associated accrual of oxidative damage to mouse skeletal muscle mitochondria. Free Radical Biology and Medicine 1998, 25:1089-1097.

1227. Masoro EJ: Caloric restriction and aging: an update. Exp Gerontol 2000, 35:299-305.

1228. Thrasivoulou C, Soubeyre V, Ridha H, Giuliani D, Giaroni C, Michael GJ, Saffrey MJ and Cowen T: Reactive oxygen species dietary restriction and neurotrophic factors in age-related loss of myenteric neurons. Aging Cell 2006, 5:247-57.

1229. Walz D: Thermodynamics of oxidation-reduction reactions and its application to bioenergetics. Biochim Biophys Acta 1979, 505:279-353.

1230. Cook Cl and Yu BP: Iron accumulation in aging: modulation by dietary restriction. Mechanisms of Ageing and Development 1998, 102:1-13.

123I. Reverter-Branchat G, Cabiscol E, Tamarit J and Ros J: Oxidative damage to specific proteins in replicative and chronological-aged Saccharomyces cerevisiae - Common targets and prevention by calorie restriction. I Biol Chem 2004, 279:31983-31989.

1232. Raffaghello L, Lee C, Safdie FM, Wei M, Madia F, Bianchi G and Longo VD: Starvation-dependent differential stress resistance protects normal but not cancer cells against highdose chemotherapy. Proc Natl Acad Sci USA 2008, 105:82 I5-20.

1233. Anisimov VN: Effects of exogenous melatonin-a review. Toxicol Pathol 2003, 31:589-603.

1234. Poeggeler B: Melatonin, aging, and age-related diseases: perspectives for prevention, intervention, and therapy. Endocrine 2005, 27:201-12.

1235. Bonilla E, Medina-Leendertz S, Villalobos V, Molero L and Bohorquez A: Paraquat-induced oxidative stress in Drosophila melanogaster: Effects of melatonin, glutathione, serotonin, minocycline, lipoic acid and ascorbic acid. Neurochemical Research 2006, 31:|425-|432.

1236. Salvemini D, Riley DP and Cuzzocrea S: SOD mimetics are coming of age. Nature Reviews Drug Discovery 2002, I:367-374.

1237. Lithgow GJ, Gill MS, Olsen A and Sampayo JN: Pharmacological intervention in invertebrate aging. Ageing Res Rev 2005, 27:213-223.

1238. Murphy CT, McCarroll SA, Bargmann Cl, Fraser A, Kamath RS, Ahringer J, Li H and Kenyon C: Genes that act downstream of DAF-I 6 to influence the lifespan of Caenorhabditis elegans. Nature 2003, 424:277-83.

1239. Dong MQ, Venable JD, Au N, Xu T, Park SK, Cociorva D, Johnson JR, Dillin A and Yates JR 3rd: Quantitative mass spectrometry identifies insulin signaling targets in $C$. elegans. Science 2007, 317:660-3.

1240. Honda $Y$ and Honda S: The daf-2 gene network for longevity regulates oxidative stress resistance and Mn-superoxide dismutase gene expression in Caenorhabditis elegans. FASEB 1999, I 3: 1385-93.

124I. Bauerova K and Bezek S: Role of reactive oxygen and nitrogen species in etiopathogenesis of rheumatoid arthritis. General Physiology and Biophysics 1999, I 8: I5-20.

1242. Aruoma OI: Free radicals, oxidative stress, and antioxidants in human health and disease. J Amer Oil Chemists Soc 1998, 75:199-212

1243. Mapp PI, Grootveld MC and Blake DR: Hypoxia, oxidative stress and rheumatoid arthritis. British Medical Bulletin 1995, 51:419-436.

1244. Gracy RW, Talent JM, Kong $Y$ and Conrad CC: Reactive oxygen species: the unavoidable environmental insult? Mut Res 1999 , 428: $17-22$.

1245. Henrotin YE, Bruckner $\mathrm{P}$ and Pujol JPL: The role of reactive oxygen species in homeostasis and degradation of cartilage. Osteoarthritis and Cartilage 2003, I I:747-755.

1246. Griffiths HR: ROS as signalling molecules in T cells evidence for abnormal redox signalling in the autoimmune disease, rheumatoid arthritis. Redox Report 2005, 10:273-280.

1247. Weinstein IM: A Correlative Study of the Erythrokinetics and Disturbances in Iron Metabolism Associated with the Anemia of Rheumatoid Arthritis. Blood 1959, I 4:950-966.

1248. Bentley DP and Williams P: Serum ferritin concentration as an index of storage iron in rheumatoid arthritis. J Clin Pathol 1974, 27:786-788.

1249. Hansen TM, Hansen NE, Birgens HS, Holund B and Lorenzen I: Serum Ferritin and the Assessment of Iron-Deficiency in Rheumatoid-Arthritis. Scandinavian Journal of Rheumatology 1983, I 2:353-359.

1250. Weber J, Werre JM, Julius HW and Marx JJ: Decreased iron absorption in patients with active rheumatoid arthritis, with and without iron deficiency. Ann Rheum Dis 1988 , 47:404-9.

125I. Vreugdenhil G, Wognum AW, Vaneijk HG and Swaak AJG: Anemia in Rheumatoid-Arthritis - the Role of Iron, Vitamin-B I2, and Folic-Acid Deficiency, and Erythropoietin Responsiveness. Annals of the Rheumatic Diseases 1990, 49:93-98.

1252. Muirden KD and Senator GB: Iron in synovial membrane in rheumatoid arthritis and other joint diseases. Ann Rheumat Dis 1968, 27:38.

1253. Rowley D, Gutteridge JMC, Blake D, Farr M and Halliwell B: Lipid peroxidation in rheumatoid arthritis - thiobarbituric acidreactive material and catalytic iron salts in synovial fluid from rheumatoid patients. Clin Sci 1984, 66:691-695.

1254. Biemond P, Swaak AJG, Vaneijk HG and Koster JF: Intraarticular ferritin-bound iron in rheumatoid arthritis - a factor that increases oxygen free radical-induced tissue destruction. Arthritis and Rheumatism 1986, 29: I 187-II93.

1255. Lipiński P and Drapier JC: Interplay between ferritin metabolism, reactive oxygen species and nitric oxide. J Biol Inorg Chem 1997, 2:559-566.

1256. Biemond P, Swaak AJG, Vaneijk HG and Koster JF: Superoxide Dependent Iron Release from Ferritin in Inflammatory Diseases. Free Radical Biology and Medicine 1988, 4:185-198.

1257. Biemond P, Swaak AJG, Beindorff CM and Koster JF: SuperoxideDependent and Superoxide-Independent Mechanisms of Iron Mobilization from Ferritin by Xanthine-Oxidase Implications for Oxygen-Free-Radical-Induced Tissue Destruction During Ischemia and Inflammation. Biochemical Journal 1986, 239:169-173.

1258. Biemond P, Swaak AJG, Penders JMA, Beindorff CM and Koster JF: Superoxide Production by Polymorphonuclear Leukocytes in Rheumatoid-Arthritis and Osteoarthritis - Invivo Inhibition by the Antirheumatic Drug Piroxicam Due to Interference with the Activation of the Nadph-Oxidase. Annals of the Rheumatic Diseases 1986, 45:249-255.

1259. Biemond P, Vaneijk HG, Swaak AJG and Koster JF: Iron Mobilization from Ferritin by Superoxide Derived from Stimulated Polymorphonuclear Leukocytes - Possible Mechanism in Inflammation Diseases. Journal of Clinical Investigation 1984, 73:1576-1579.

1260. Mladěnka $P$, Šimůnek $T$, Hübl $M$ and Hrdina $R$ : The role of reactive oxygen and nitrogen species in cellular iron metabolism. Free Radic Res 2006, 40:263-72. 
126I. Harris LR, Cake MH and Macey D): Iron release from ferritin and its sensitivity to superoxide ions differs among vertebrates. Biochem J 1994, 301:385-389.

1262. Fairburn K, Grootveld M, Ward RJ, Abiuka C, Kus M, Williams RB, Winyard PG and Blake DR: Alpha-Tocopherol, Lipids and Lipoproteins in Knee-Joint Synovial-Fluid and Serum from Patients with Inflammatory Joint Disease. Clinical Science 1992, 83:657-664.

1263. Dombrecht EJ, Cos P, Berghe Vanden D, Van Offel JF, Schuerwegh AJ, Bridts CH, Stevens WJ and De Clerck LS: Selective in vitro antioxidant properties of bisphosphonates. Biochem Biophys Res Commun 2004, 3 I4:675-80.

1264. Ostrakhovitch EA and Afanas'ev IB: Oxidative stress in rheumatoid arthritis leukocytes: suppression by rutin and other antioxidants and chelators. Biochem Pharmacol 200I, 62:743-746.

1265. Hazes JMW, Dijkmans BAC, Vandenbroucke JP, Devries RRP and Cats A: Pregnancy and the risk of developing rheumatoid arthritis. Arthritis and Rheumatism 1990, 33:1770-1775.

1266. Østensen $M$ and Husby G: A prospective clinical study of the effect of pregnancy on rheumatoid arthritis and ankylosing spondylitis. Arthritis and Rheumatism 1983, 26: II55-II59.

1267. Da Silva JAP and Spector TD: The role of pregnancy in the course and etiology of rheumatoid arthritis. Clin Rheumat 1992, I I: I89-194.

1268. O'Keeffe ST: Restless legs syndrome - A review. Archives of Internal Medicine 1996, I 56:243-248.

1269. Ondo W, Tan EK and Mansoor J: Rheumatologic serologies in secondary restless legs syndrome. Movement Disorders 2000, I 5:321-323.

1270. Trenkwalder C, Paulus $W$ and Walters AS: The restless legs syndrome. Lancet Neurology 2005, 4:465-475

127I. Feelders RA, Kuiper-Kramer EPA and van Eijk HG: Structure, function and clinical significance of transferrin receptors. Clinical Chemistry and Laboratory Medicine 1999, 37:I-I0.

1272. Ahluwalia N: Diagnostic utility of serum transferrin receptors measurement in assessing iron status. Nutrition Reviews 1998, 56:133-141.

1273. Frostegård J: SLE, atherosclerosis and cardiovascular disease. J Intern Med 2005, 257:485-95.

1274. Mascitelli L and Pezzetta F: High iron stores and cardiovascular disease in systemic lupus erythematosus. J Intern Med 2005 258:584.

1275. Harvey AM, Shulman LE, Tumulty PA, Conley CL and Schoenrich EH: Systemic lupus erythematosus: review of the literature and clinical analysis of 138 cases. Medicine (Baltimore) 1954, 33:291-437.

1276. Johnson RT and Richardson EP: The neurological manifestations of systemic lupus erythematosus. Medicine (Baltimore) $1968,47: 337-69$

1277. Feng PH, Cheah PS and Lee YK: Mortality in systemic lupus erythematosus: a I 0-year review. Br Med J 1973, 4:772-4.

1278. Matthay RA, Schwarz MI, Petty TL, Stanford RE, Gupta RC, Sahn SA and Steigerwald JC: Pulmonary manifestations of systemic lupus erythematosus: review of twelve cases of acute lupus pneumonitis. Medicine (Baltimore) 1975, 54:397-409.

1279. O'Loughlin S, Schroeter AL and Jordon RE: Chronic urticarialike lesions in systemic lupus erythematosus. A review of 12 cases. Arch Dermatol 1978, I |4:879-83.

1280. Hoffman BI and Katz WA: The gastrointestinal manifestations of systemic lupus erythematosus: a review of the literature. Semin Arthritis Rheum 1980, 9:237-47.

128I. Reynolds JC, Inman RD, Kimberly RP, Chuong JH, Kovacs JE and Walsh MB: Acute pancreatitis in systemic lupus erythematosus: report of twenty cases and a review of the literature. Medicine (Baltimore) 1982, 61:25-32.

1282. Tan EM, Cohen AS, Fries JF, Masi AT, McShane DJ, Rothfield NF Schaller JG, Talal $N$ and Winchester RJ: The 1982 revised criteria for the classification of systemic lupus erythematosus. Arthritis Rheum 1982, 25: I27I-7.

1283. Levine SR, Joseph R, D'Andrea G and Welch KM: Migraine and the lupus anticoagulant. Case reports and review of the literature. Cephalalgia 1987, 7:93-9.

1284. Crowson AN and Magro C: The cutaneous pathology of lupus erythematosus: a review. I Cutan Pathol 200I, 28: I-23.

1285. Karrar A, Sequeira W and Block JA: Coronary artery disease in systemic lupus erythematosus: A review of the literature. Semin Arthritis Rheum 2001, 30:436-43.

1286. Ad hoc committee on systemic lupus erythematosus response criteria for fatigue: Measurement of fatigue in systemic lupus erythematosus: a systematic review. Arthritis Rheum 2007, 57:1348-57.

1287. Zhang X, Jin M, Wu H, Nadasdy T, Nadasdy G, Harris N, GreenChurch K, Nagaraja H, Birmingham DJ, Yu CY, Hebert LA and Rovin $\mathrm{BH}$ : Biomarkers of lupus nephritis determined by serial urine proteomics. Kidney Int 2008, 74:799-807.

1288. Lim MK, Lee CK, Ju YS, Cho YS, Lee MS, Yoo B and Moon HB: Serum ferritin as a serologic marker of activity in systemic lupus erythematosus. Rheumatol Int 2001, 20:89-93.

1289. Abou-Raya A, el-Hallous D and Fayed H: 8-Isoprostaglandin F2 alpha: a potential index of lipid peroxidation in systemic lupus erythematosus. Clin Invest Med 2004, 27:306-I I.

1290. Cutolo M and Otsa K: Review: vitamin D, immunity and lupus. Lupus 2008, 17:6-10.

1291. Li HF: Are statins analogues of vitamin D? Lancet 2006, 368: $1233-4$

1292. Wu-Wong JR, Nakane M, Ma J and Ju TR: Statins do not directly activate vitamin D receptor. I Thromb Haemost 2007, 5:4I 5-6.

1293. Lawman S, Mauri C, Jury EC Cook HT and Ehrenstein MR Atorvastatin inhibits autoreactive $B$ cell activation and delays lupus development in New Zealand black/white F I mice. J Immunol 2004, I 73:764I-6.

1294. Jury EC, Isenberg DA, Mauri C and Ehrenstein MR: Atorvastatin restores Lck expression and lipid raft-associated signaling in $T$ cells from patients with systemic lupus erythematosus. Immunol 2006, I77:74I6-22.

1295. Swanson DR: Undiscovered public knowledge. Library Quarterly 1986, 56:103-II8.

1296. Swanson DR, Smalheiser NR and Torvik VI: Ranking indirect connections in literature-based discovery: The role of medical subject headings. J Amer Soc Inf Sci Technol 2006, 57:|427-|439.

1297. Andreadis AA, Hazen SL, Comhair SAA and Erzurum SC: Oxidative and nitrosative events in asthma. Free Radical Biology and Medicine 2003, 35:213-225

1298. Greene LS: Asthma and oxidant stress - nutritional, environmental, and genetic risk factors. J Amer Coll Nutrition 1995, I4:317-324.

1299. Han JY, Takeshita K and Utsumi H: Noninvasive detection of hydroxyl radical generation in lung by diesel exhaust particles. Free Radic Biol Med 2001, 30:516-25.

1300. Kocyigit A, Armutcu F, Gurel A and Ermis B: Alterations in plasma essential trace elements selenium, manganese, zinc, copper, and iron concentrations and the possible role of these elements on oxidative status in patients with childhood asthma. Biological Trace Element Research 2004 97:3|-4I.

I30I. Lih-Brody L, Powell SR, Collier KP, Reddy GM, Cerchia R, Kahn E, Weissman GS, Katz S, Floyd RA, McKinley MJ, Fisher SE and Mullin GE. Increased oxidative stress and decreased antioxidant defenses in mucosa of inflammatory bowel disease. Dig Dis Sci 1996, 41:2078-86.

1302. Kruidenier L and Verspaget HW: Review article: oxidative stress as a pathogenic factor in inflammatory bowel disease-radicals or ridiculous? Aliment Pharmacol Ther 2002 , 16:1997-2015.

1303. Kruidenier L, Kuiper I, Lamers CB and Verspaget HW. Intestinal oxidative damage in inflammatory bowel disease: semiquantification, localization, and association with mucosal antioxidants. J Pathol 2003, 201:28-36.

1304. Martin AR, Villegas I, La Casa C and de la Lastra CA: Resveratrol, a polyphenol found in grapes, suppresses oxidative damage and stimulates apoptosis during early colonic inflammation in rats. Biochem Pharmacol 2004, 67:1399-410.

1305. Seril DN, Liao J, Yang GY and Yang CS: Oxidative stress and ulcerative colitis-associated carcinogenesis: studies in humans and animal models. Carcinogenesis 2003, 24:353-62.

1306. Gisbert JP and Gomollón F: Common misconceptions in the diagnosis and management of anemia in inflammatory bowel disease. Am J Gastroenterol 2008, I 03:1299-307.

1307. Semrin G, Fishman DS, Bousvaros A, Zholudev A, Saunders AC Correia CE, Nemeth E, Grand RJ and Weinstein DA: Impaired intestinal iron absorption in Crohn's disease correlates with disease activity and markers of inflammation. Inflamm Bowel Dis 2006, 12:1101-6.

1308. Zaahl MG, Winter TA, Warnich L and Kotze MJ: The -237C->T promoter polymorphism of the SLCIIAI gene is associated with a protective effect in relation to inflammatory bowel disease in the South African population. Int J Colorectal Dis 2006, 21:402-8. 
1309. Bartels U, Pedersen NS and Jarnum S: Iron absorption and serum ferritin in chronic inflammatory bowel disease. Scand J Gastroenterol 1978, I 3:649-56.

1310. Thomson AB, Brust R, Ali MA, Mant MJ and Valberg LS: Iron deficiency in inflammatory bowel disease. Diagnostic efficacy of serum ferritin. Am J Dig Dis 1978, 23:705-9.

I3II. Oldenburg B, Koningsberger JC, Van Berge Henegouwen GP, Van Asbeck BS and Marx J]: Iron and inflammatory bowel disease. Aliment Pharmacol Ther 200I, I 5:429-38.

1312. Gasche C, Lomer MC, Cavill I and Weiss G: Iron, anaemia, and inflammatory bowel diseases. Gut 2004, 53: I 190-7.

1313. de Silva AD, Tsironi E, Feakins RM and Rampton DS: Efficacy and tolerability of oral iron therapy in inflammatory bowel disease: a prospective, comparative trial. Aliment Pharmacol Ther 2005, 22:1097-105.

13|4. Guagnozzi D, Severi C, lalongo P, Viscido A, Patrizi F, Testino G, Vannella L, Labriola R, Strom R and Caprilli R: Ferritin as a simple indicator of iron deficiency in anemic IBD patients. Inflamm Bowel Dis 2006, I 2: I50-I.

1315. Gasche C, Berstad A, Befrits R, Beglinger C, Dignass A, Erichsen K, Gomollon F, Hjortswang $\mathrm{H}$, Koutroubakis I, Kulnigg S, Oldenburg B, Rampton D, Schroeder O, Stein J, Travis S and Van Assche G: Guidelines on the diagnosis and management of iron deficiency and anemia in inflammatory bowel diseases. Inflamm Bowel Dis 2007, I 3: I545-53.

1316. Rattner $A$ and Nathans J: Macular degeneration: recent advances and therapeutic opportunities. Nat Rev Neurosci 2006, 7:860-72.

1317. Tielsch JM, Javitt JC, Coleman A, Katz J and Sommer A: The prevalence of blindness and visual impairment among nursing home residents in Baltimore. N Engl J Med 1995, 332:1205-9.

1318. Harvey PT: Common eye diseases of elderly people: identifying and treating causes of vision loss. Gerontology 2003, 49: I-II.

1319. Fine SL: Age-related macular degeneration 1969-2004: a 35year personal perspective. Am J Ophthalmol 2005, 139:405-20.

1320. Lotery A and Trump D: Progress in defining the molecular biology of age related macular degeneration. Hum Genet 2007, I 22:219-36

I32I. Anderson DH, Mullins RF, Hageman GS and Johnson LV: A role for local inflammation in the formation of drusen in the aging eye. Am J Ophthalmol 2002, I34:4II-3I.

1322. Hahn P, Ying GS, Beard J and Dunaief JL: Iron levels in human retina: sex difference and increase with age. Neuroreport 2006, I7:1803-6.

1323. Cai J, Nelson KC, Wu M, Sternberg P Jr and Jones DP: Oxidative damage and protection of the RPE. Prog Retin Eye Res 2000, | 9:205-2 |

1324. Richer S, Rudy D, Statkute L, Karofty K and Frankowski J: Serum iron, transferrin saturation, ferritin, and dietary data in age-related macular degeneration. Am J Ther 2002, 9:25-8.

1325. Hahn P, Milam AH and Dunaief JL: Maculas affected by agerelated macular degeneration contain increased chelatable iron in the retinal pigment epithelium and Bruch's membrane. Arch Ophthalmol 2003, I 2 I: 1099-105.

1326. Hahn P, Qian Y, Dentchev T, Chen L, Beard J, Harris ZL and Dunaief JL: Disruption of ceruloplasmin and hephaestin in mice causes retinal iron overload and retinal degeneration with features of age-related macular degeneration. Proc Natl Acad Sci USA 2004, I 0 I: | 3850-5.

1327. Dunaief JL: Iron induced oxidative damage as a potential factor in age-related macular degeneration: The Cogan Lecture. Invest Ophthalmol Vis Sci 2006, 47:4660-4664.

1328. He X, Hahn P, lacovelli J, Wong R, King C, Bhisitkul R, MassaroGiordano $M$ and Dunaief JL: Iron homeostasis and toxicity in retinal degeneration. Prog Retin Eye Res 2007, 26:649-73.

1329. Wong RW, Richa DC, Hahn P, Green WR and Dunaief JL: Iron toxicity as a potential factor in AMD. Retina 2007, 27:997-1003.

1330. Voloboueva LA, Killilea DW, Atamna $\mathrm{H}$ and Ames BN: N-tertbutyl hydroxylamine, a mitochondrial antioxidant, protects human retinal pigment epithelial cells from iron overload: relevance to macular degeneration. FASEB J 2007, 2I:4077-86

I33I. Tan JS, Wang J, Flood V, Rochtchina E, Smith W and Mitchell P: Dietary antioxidants and the long-term incidence of agerelated macular degeneration: the Blue Mountains Eye Study. Ophthalmology 2008, I I 5:334-4I
1332. Wilson DS and Szostak JW: In vitro selection of functional nucleic acids. Annu Rev Biochem 1999, 68:6II-647.

1333. Nimjee SM, Rusconi CP and Sullenger BA: Aptamers: an emerging class of therapeutics. Annu Rev Med 2005, 56:555-83.

1334. Famulok M, Hartig JS and Mayer G: Functional aptamers and aptazymes in biotechnology, diagnostics, and therapy. Chem Rev 2007, 107:37|5-43.

1335. Chapman JA and Beckey C: Pegaptanib: a novel approach to ocular neovascularization. Ann Pharmacother 2006, 40: | 322-6.

1336. Kourlas $H$ and Schiller DS: Pegaptanib sodium for the treatment of neovascular age-related macular degeneration: a review. Clin Ther 2006, 28:36-44.

1337. Zhou B and Wang B: Pegaptanib for the treatment of agerelated macular degeneration. Exp Eye Res 2006, 83:6I5-9.

1338. Virgili G, Do DV, Bressler NM and Menchini U: New therapies for neovascular age-related macular degeneration: critical appraisal of the current evidence. Acta Ophthalmol Scand 2007, 85:6-20

1339. Takeda AL, Colquitt J, Clegg AJ and Jones J: Pegaptanib and ranibizumab for neovascular age-related macular degeneration: a systematic review. Br J Ophthalmol 2007, 9 I : I I 77-82.

1340. Apte RS: Pegaptanib sodium for the treatment of agerelated macular degeneration. Expert Opin Pharmacother 2008, 9:499-508.

[34I. Trenam CW, Dabbagh AJ, Morris Cl and Blake DR: Skin inflammation induced by reactive oxygen species (ROS): an in vivo model. Br J Dermatol I991, I 25:325-9.

1342. Trouba KJ, Hamadeh HK, Amin RP and Germolec DR: Oxidative stress and its role in skin disease. Antioxidants \& Redox Signaling 2002, 4:665-673.

1343. Wojas-Pelc A and Marcinkiewicz J: What is a role of haeme oxygenase-I in psoriasis? Current concepts of pathogenesis. Int J Exp Pathol 2007, 88:95-102.

1344. Trenam CW, Blake DR and Morris C): Skin inflammation reactive oxygen species and the role of iron. I Invest Dermatol 1992, 99:675-682.

1345. Trenam CW, Dabbagh AJ, Blake DR and Morris C]: The role of iron in an acute model of skin inflammation induced by reactive oxygen species (ROS). $\mathrm{Br} J$ Dermatol I992, 1 26:250-256.

1346. Hider RC, Singh S and Porter JB: Iron chelating agents with clinical potential. Proc R Soc Ed B 1992, 99:137-168.

1347. Kim KY, Schumacher HR, Hunsche E, Wertheimer Al and Kong SX: A literature review of the epidemiology and treatment of acute gout. Clinical Therapeutics 2003, 25:1593-1617.

1348. Davies KJ, Sevanian A, Muakkassah-Kelly SF and Hochstein P: Uric acid-iron ion complexes. A new aspect of the antioxidant functions of uric acid. Biochem J 1986, 235:747-54.

1349. Ghio AJ, Kennedy TP, Rao G, Cooke CL, Miller MJ and Hoidal JR: Complexation of iron cation by sodium urate crystals and gouty inflammation. Arch Biochem Biophys 1994, 3 I 3:2 I 5-22 I.

1350. Ghio AJ, Ford ES, Kennedy TP and Hoidal JR: The association between serum ferritin and uric acid in humans. Free Radical Research 2005, 39:337-342.

I35I. Facchini FS: Near-iron deficiency-induced remission of gouty arthritis. Rheumatology 2003, 42:1550-1555.

1352. Poli R and Langdon WB: On the search properties of different crossover operators in genetic programming. Proc. 3rd Conf Genetic Programming GP'98 Morgan Kaufmann, Madison, WI; 1998, 293-301.

1353. Brittenham GM: Iron chelators and iron toxicity. Alcohol 2003, 30: $|5|-8$.

1354. Tung BY, Emond MJ, Bronner MP, Raaka SD, Cotler SJ and Kowdley KV: Hepatitis C, iron status, and disease severity: relationship with HFE mutations. Gastroenterology 2003 124:318-26.

1355. Corradini E, Ferrara F and Pietrangelo A: Iron and the liver. Pediatr Endocrinol Rev 2004, 2(Suppl 2):245-8.

1356. Rigamonti C, Andorno S, Maduli E, Capelli F, Boldorini R and Sartori M: Gender and liver fibrosis in chronic hepatitis: the role of iron status. Aliment Pharmacol Ther 2005, 2 I:|445-5I.

1357. Philippe MA, Ruddell RG and Ramm GA: Role of iron in hepatic fibrosis: one piece in the puzzle. World J Gastroenterol 2007, I3:4746-54.

1358. Kohgo $Y$, Ohtake T, lkuta K, Suzuki $Y$, Torimoto $Y$ and Kato J: Dysregulation of systemic iron metabolism in alcoholic liver diseases. J Gastroenterol Hepatol 2008, 23(SuppI I):S78-8I. 
1359. Ramm GA and Ruddell RG: Hepatotoxicity of iron overload: Mechanisms of iron-induced hepatic fibrogenesis. Seminars in Liver Disease 2005, 25:433-449.

1360. McCullough KD and Bartfay WJ: The dose-dependent effects of chronic iron overload on the production of oxygen free radicals and vitamin $E$ concentrations in the liver of a murine model. Biol Res Nurs 2007, 8:300-4.

I36I. Petrak J, Myslivcova D, Man P, Cmejla R, Cmejlova J, Vyoral D, Elleder $M$ and Vulpe CD: Proteomic analysis of hepatic iron overload in mice suggests dysregulation of urea cycle, impairment of fatty acid oxidation, and changes in the methylation cycle. Am J Physiol Gastrointest Liver Physiol 2007, 292: GI490-8.

1362. Aigner E, Theurl I, Theurl M, Lederer D, Haufe H, Dietze O, Strasser M, Datz $C$ and Weiss G: Pathways underlying iron accumulation in human nonalcoholic fatty liver disease. Am J Clin Nutr 2008, 87:1374-83.

1363. Chen CJ, Yu MW and Liaw YF: Epidemiological characteristics and risk factors of hepatocellular carcinoma. I Gastroenterol Hepatol 1997, I 2:S294-308.

1364. Asare GA, Mossanda KS, Kew MC, Paterson AC, Kahler-Venter CP and Siziba K: Hepatocellular carcinoma caused by iron overload: A possible mechanism of direct hepatocarcinogenicity. Toxicology 2006, 2 I 9:4I-52.

1365. Kato J, Kobune M, Nakamura T, Kuroiwa G, Takada K, Takimoto R, Sato Y, Fujikawa K, Takahashi M, Takayama T, Ikeda T and Niitsu Y: Normalization of elevated hepatic 8-hydroxy-2'-deoxyguanosine levels in chronic hepatitis $\mathbf{C}$ patients by phlebotomy and low iron diet. Cancer Res 200I, 6l:8697-702.

1366. Ritter C, Reinke A, Andrades M, Martins MR, Rocha J, MennaBarreto S, Quevedo J, Moreira JC and Dal-Pizzol F: Protective effect of $\mathbf{N}$-acetylcysteine and deferoxamine on carbon tetrachloride-induced acute hepatic failure in rats. Crit Care Med 2004, 32:2079-83.

1367. Bridle K, Cheung TK, Murphy T, Walters M, Anderson G, Crawford DG and Fletcher LM: Hepcidin is down-regulated in alcoholic liver injury: implications for the pathogenesis of alcoholic liver disease. Alcohol Clin Exp Res 2006, 30:106-12.

1368. Antó JM, Vermeire P, Vestbo J and Sunyer J: Epidemiology of chronic obstructive pulmonary disease. European Respiratory Journal 2001, 17:982-994.

1369. White AJ, Gompertz S and Stockley RA: Chronic obstructive pulmonary disease .6: The aetiology of exacerbations of chronic obstructive pulmonary disease. Thorax 2003 58:73-80.

1370. Tsoumakidou $M$ and Siafakas NM: Novel insights into the aetiology and pathophysiology of increased airway inflammation during COPD exacerbations. Respiratory Research 2006, 7:.

137I. Sapey E and Stockley RA: COPD exacerbations 2: Aetiology. Thorax 2006, 61:250-258.

1372. Rodriguez-Roisin R: Toward a consensus definition for COPD exacerbations. Chest 2000, I I7:398S-40IS

1373. Weinberg ED: Tobacco smoke iron: an initiator/promoter of multiple diseases. Biometals 2008 in press.

1374. Rahman I, van Schadewijk AA, Crowther AJ, Hiemstra PS, Stolk J, MacNee W and De Boer WI: 4-Hydroxy-2-nonenal, a specific lipid peroxidation product, is elevated in lungs of patients with chronic obstructive pulmonary disease. Am J Respir Crit Care Med 2002, I 66:490-5.

1375. Langen RC], Korn SH and Wouters EFM: ROS in the local and systemic pathogenesis of COPD. Free Rad Biol Med 2003 35:226-235.

1376. Barnes PJ: Mediators of chronic obstructive pulmonary disease. Pharmacol Rev 2004, 56:515-548.

1377. Santos MC, Oliveira AL, Viegas-Crespo AM, Vicente L, Barreiros A, Monteiro P, Pinheiro T and De Almeida AB: Systemic markers of the redox balance in chronic obstructive pulmonary disease. Biomarkers 2004, 9:46I-469.

1378. Kinnula VL: Focus on antioxidant enzymes and antioxidant strategies in smoking related airway diseases. Thorax 2005 , 60:693-700.

1379. Rytila P, Rehn T, llumets $H$, Rouhos A, Sovijarvi A, Myllarniemi M and Kinnula VL: Increased oxidative stress in asymptomatic current chronic smokers and GOLD stage 0 COPD. Respiratory Research 2006, 7:.

1380. Stevenson CS, Koch LG and Britton SL: Aerobic capacity, oxidant stress, and chronic obstructive pulmonary disease - A new take on an old hypothesis. Pharmacol Therapeut 2006, | | 0:7|-82.
|38I. Kinnula VL, llumets H, Myllarnlemi M, Sovijarvi A and Rytila P: 8Isoprostane as a marker of oxidative stress in nonsymptomatic cigarette smokers and COPD. European Respiratory Journal 2007, 29:5I-55.

1382. Huang $X, \mathrm{Li}$ WH, Attfield MD, Nadas $A$, Frenkel $K$ and Finkelman RB: Mapping and prediction of coal workers' pneumoconiosis with bioavailable iron content in the bituminous coals. Environmental Health Perspectives 2005, II 3:964-968.

1383. Mutti A, Corradi M, Goldoni M, Vettori MV, Bernard A and Apostoli P: Exhaled metallic elements and serum pneumoproteins in asymptomatic smokers and patients with COPD or asthma. Chest 2006, I29:| 288-1297.

1384. Carter JD, Ghio AJ, Samet JM and Devlin RB: Cytokine production by human airway epithelial cells after exposure to an air pollution particle is metal-dependent. Toxicol Appl Pharmacol 1997, |46:180-8.

1385. Corhay JL, Weber G, Bury T, Mariz S, Roelandts I and Radermecker MF: Iron content in human alveolar macrophages. Eur Resp J 1992, 5:804-809.

1386. Stites SW, Nelson ME and Wesselius LJ: Transferrin concentrations in serum and lower respiratory tract fluid of mechanically ventilated patients with COPD or ARDS. Chest 1995, 107:1681-1685.

1387. Southorn PA and Powis G: Free radicals in medicine .2. involvement in human disease. Mayo Clinic Proceedings 1988, 63:390-408.

1388. Quinlan GJ, Lamb NJ, Tilley R, Evans TW and Gutteridge JMC: Plasma hypoxanthine levels in ARDS: Implications for oxidative stress, morbidity, and mortality. Amer I Resp Crit Care Med 1997, 155:479-484.

1389. Quinlan G], Evans TW and Gutteridge JMC: Oxidative damage to plasma proteins in adult respiratory distress syndrome. Free Radical Research 1994, 20:289-298.

1390. Gutteridge JMC and Quinlan GJ: Antioxidant protection against organic and inorganic oxygen radicals by normal human plasma - the important primary role for ironbinding and iron-oxidizing proteins. Biochim Biophys Acta 1993 , I I 56: I44-150.

1391. Reid DW, Lam OT, Schneider $\mathrm{H}$ and Walters EH: Airway iron and iron-regulatory cytokines in cystic fibrosis. European Respiratory Journal 2004, 24:286-29l.

1392. Doll $R$ and Hill $A B$ : Smoking and carcinoma of the lung; preliminary report. $\mathrm{Br}$ Med J 1950, 2:739-48.

1393. Peto R, Darby S, Deo H, Silcocks P, Whitley E and Doll R: Smoking, smoking cessation, and lung cancer in the UK since 1950: combination of national statistics with two case-control studies. BMJ 2000, 32I:323-9.

1394. Doll $R$ and Hill $A B$ : The mortality of doctors in relation to their smoking habits: a preliminary report. I 954. BM] 2004, 328: I529-33.

1395. Doll R, Peto R, Boreham J and Sutherland I: Mortality from cancer in relation to smoking: 50 years observations on British doctors. $\mathrm{Br} /$ Cancer 2005, 92:426-9.

1396. Thompson AB, Bohling T, Heires A, Linder J and Rennard SI: Lower respiratory tract iron burden is increased in association with cigarette smoking. J Lab Clin Med 1991, I I 7:493-9.

1397. Dreher KL, Jaskot RH, Lehmann JR, Richards JH, McGee JK, Ghio AJ and Costa DL: Soluble transition metals mediate residual oil fly ash induced acute lung injury. J Toxicol Environ Health 1997, 50:285-305.

1398. Mateos F, Brock JH and Perez-Arellano JL: Iron metabolism in the lower respiratory tract. Thorax 1998, 53:594-600.

1399. Boelaert JR, Gomes MS and Gordeuk VR: Smoking, iron, and tuberculosis. Lancet 2003, 362: I 243-4.

1400. Ghio AJ, Carter JD, Richards JH, Richer LD, Grissom CK and Elstad MR: Iron and iron-related proteins in the lower respiratory tract of patients with acute respiratory distress syndrome. Crit Care Med 2003, 3 I:395-400.

|40 I. Ernst P. Review article: the role of inflammation in the pathogenesis of gastric cancer. Aliment Pharmacol Ther 1999, I3 (Suppl I): I3-18.

1402. Kelloff G], Crowell JA, Steele VE, Lubet RA, Malone WA Boone CW, Kopelovich L, Hawk ET, Lieberman R, Lawrence JA, Ali I, Viner JL and Sigman CC: Progress in cancer chemoprevention: development of diet-derived chemopreventive agents. I Nutr 2000, I30:467S-47IS.

1403. Balkwill $F$ and Mantovani $A$ : Inflammation and cancer: back to Virchow? Lancet 200I, 357:539-45. 
1404. Coussens LM and Werb Z: Inflammation and cancer. Nature 2002, 420:860-7.

1405. Aggarwal BB: Nuclear factor-kappaB: the enemy within. Cancer Cell 2004, 6:203-8.

1406. Vakkila J and Lotze MT: Inflammation and necrosis promote tumour growth. Nat Rev Immunol 2004, 4:64I-8.

1407. Balkwill F, Charles KA and Mantovani A: Smoldering and polarized inflammation in the initiation and promotion of malignant disease. Cancer Cell 2005, 7:2 II-7.

1408. Karin $M$ and Greten FR: NF-kappaB: linking inflammation and immunity to cancer development and progression. Nat Rev Immunol 2005, 5:749-59.

1409. Li Q, Withoff $\mathrm{S}$ and Verma IM: Inflammation-associated cancer: NF-kappaB is the Iynchpin. Trends Immunol 2005, 26:318-25.

1410. Steele VE and Kelloff GJ: Development of cancer chemopreventive drugs based on mechanistic approaches. Mutat Res 2005, 591:16-23.

14II. Aggarwal BB, Shishodia S, Sandur SK, Pandey MK and Sethi G: Inflammation and cancer: how hot is the link? Biochem Pharmacol 2006, 72:1605-2I.

1412. Karin M: NF-kappaB and cancer: mechanisms and targets. Mol Carcinog 2006, 45:355-6I.

14|3. Kelloff GJ, Lippman SM, Dannenberg AJ, Sigman CC, Pearce HL, Reid BJ, Szabo E, Jordan VC, Spitz MR, Mills GB, Papadimitrakopoulou VA, Lotan R, Aggarwal BB, Bresalier RS, Kim J, Arun B, Lu KH, Thomas ME, Rhodes HE, Brewer MA, Follen M, Shin DM, Parnes HL, Siegfried JM, Evans AA, Blot WJ, Chow WH, Blount PL, Maley CC, Wang KK, Lam S, Lee J], Dubinett SM, Engstrom PF, Meyskens FL Jr, O'Shaughnessy J, Hawk ET, Levin B, Nelson WG and Hong WK: Progress in chemoprevention drug development: the promise of molecular biomarkers for prevention of intraepithelial neoplasia and cancer-a plan to move forward. Clin Cancer Res 2006, 1 2:366I-97.

14|4. Rakoff-Nahoum S: Why cancer and inflammation? Yale J Biol Med 2006, 79:123-30.

14I5. Yoshimura A: Signal transduction of inflammatory cytokines and tumor development. Cancer Sci 2006, 97:439-47.

1416. Hussain SP and Harris CC: Inflammation and cancer: an ancient link with novel potentials. Int J Cancer 2007, | 21:2373-80.

1417. Allavena P, Garlanda C, Borrello MG, Sica A and Mantovani A: Pathways connecting inflammation and cancer. Curr Opin Genet Dev 2008, 18:3-10.

1418. Borrello MG, Degl'Innocenti D and Pierotti MA: Inflammation and cancer: the oncogene-driven connection. Cancer Lett 2008, 267:262-70.

1419. Haverkamp J, Charbonneau B and Ratliff TL: Prostate inflammation and its potential impact on prostate cancer: a current review. I Cell Biochem 2008, I03:1344-53.

1420. Ishikawa K, Takenaga K, Akimoto M, Koshikawa N, Yamaguchi A, Imanishi H, Nakada K, Honma $Y$ and Hayashi J: ROS-generating mitochondrial DNA mutations can regulate tumor cell metastasis. Science 2008, 320:66|-4.

142 I. Karin M: The IkappaB kinase - a bridge between inflammation and cancer. Cell Res 2008, 18:334-42.

1422. Maeda S and Omata M: Inflammation and cancer: role of nuclear factor-kappaB activation. Cancer Sci 2008, 99:836-42.

1423. Mantovani A, Allavena P, Sica A and Balkwill F: Cancer-related inflammation. Nature 2008, 454:436-44.

1424. Sica A, Allavena $P$ and Mantovani A: Cancer related inflammation: the macrophage connection. Cancer Lett 2008, 267:204-15.

1425. Vasto S, Carruba G, Lio D, Colonna-Romano G, Di Bona D, Candore $G$ and Caruso $C$ : Inflammation, ageing and cancer. Mech Ageing Dev 2008.

1426. Baron JA and Sandler RS: Nonsteroidal anti-inflammatory drugs and cancer prevention. Annu Rev Med 2000, 51:5II-23.

1427. Rostom A, Dube C, Lewin G, Tsertsvadze A, Barrowman N, Code C, Sampson M and Moher D: Nonsteroidal antiinflammatory drugs and cyclooxygenase-2 inhibitors for primary prevention of colorectal cancer: a systematic review prepared for the U.S. Preventive Services Task Force. Ann Intern Med 2007, 146:376-89.

1428. Marshall BJ and Warren JR: Unidentified curved bacilli in the stomach of patients with gastritis and peptic ulceration. Lancet 1984, I:|3||-13|5.
1429. Davey HM and Kell DB: Flow cytometry and cell sorting of heterogeneous microbial populations: the importance of single-cell analysis. Microbiol Rev 1996, 60:64I-696.

1430. Marshall B: Helicobacter connections. ChemMedChem 2006, I:783-802.

143I. Rakba N, Aouad F, Henry C, Caris C, Morel I, Baret P, Pierre JL, Brissot P, Ward Rj, Lescoat G and Crichton RR: Iron mobilisation and cellular protection by a new synthetic chelator 0 Trensox. Biochem Pharmacol 1998, 55:1797-806.

1432. Le NTV and Richardson DR: The role of iron in cell cycle progression and the proliferation of neoplastic cells. Biochimica Et Biophysica Acta-Reviews on Cancer 2002, 1603:31-46.

1433. Buss JL, Torti FM and Torti SV: The role of iron chelation in cancer therapy. Curr Med Chem 2003, 10:1021-34.

1434. Lovejoy DB and Richardson DR: Iron chelators as antineoplastic agents: Current developments and promise of the PIH class of chelators. Current Medicinal Chemistry 2003, 10:1035-1049.

1435. Buss JL, Greene BT, Turner J, Torti FM and Torti SV: Iron chelators in cancer chemotherapy. Curr Top Med Chem 2004, 4:1623-35.

1436. Hanai J, Mammoto T, Seth P, Mori K, Karumanchi SA, Barasch J and Sukhatme VP: Lipocalin 2 diminishes invasiveness and metastasis of Ras-transformed cells. J Biol Chem 2005, 280: $|364|-7$.

1437. Kalinowski DS and Richardson DR: The evolution of iron chelators for the treatment of iron overload disease and cancer. Pharmacol Rev 2005, 57:547-83.

1438. Pahl PMB and Horwitz LD: Cell permeable iron chelators as potential cancer chemotherapeutic agents. Cancer Invest 2005, 23:683-69I.

1439. Nie GJ, Chen GH, Sheftel AD, Pantopoulos K and Ponka P: In vivo tumor growth is inhibited by cytosolic iron deprivation caused by the expression of mitochondrial ferritin. Blood 2006, I08:2428-2434.

1440. Edgren G, Nyren $O$ and Melbye M: Cancer as a ferrotoxic disease: are we getting hard stainless evidence? J Natl Cancer Inst 2008, 100:976-7.

|44 I. Huang X: Does iron have a role in breast cancer? Lancet Oncol 2008, 9:803-7.

1442. Zacharski LR, Chow BK, Howes PS, Shamayeva G, Baron JA Dalman RL, Malenka DJ, Ozaki CK and Lavori PW: Decreased cancer risk after iron reduction in patients with peripheral arterial disease: results from a randomized trial. I Natl Cancer Inst 2008, 100:996-1002

1443. Lim R, Ahmed N, Borregaard N, Riley C, Wafai R, Thompson EW, Quinn MA and Rice GE: Neutrophil gelatinase-associated lipocalin (NGAL) an early-screening biomarker for ovarian cancer: NGAL is associated with epidermal growth factorinduced epithelio-mesenchymal transition. Int J Cancer 2007, 120:2426-2434.

1444. lannetti A, Pacifico F, Acquaviva R, Lavorgna A, Crescenzi E, Vascotto C, Tell G, Salzano AM, Scaloni A, Vuttariello E, Chiappetta G, Formisano $S$ and Leonardi A: The neutrophil gelatinase-associated lipocalin (NGAL), a NF-\{kappa\}Bregulated gene, is a survival factor for thyroid neoplastic cells. Proc Natl Acad Sci USA 2008, I05: | 4058-63.

1445. Nelson RL, Davis FG, Sutter E, Sobin LH, Kikendall JW and Bowen P: Body iron stores and risk of colonic neoplasia. J Natl Cancer Inst 1994, 86:455-60.

1446. Okada S: Iron-induced tissue damage and cancer: The role of reactive oxygen species-free radicals. Pathol Int 1996, 46:3II-332

1447. Toyokuni S: Iron-induced carcinogenesis: The role of redox regulation. Free Radical Biology and Medicine 1996, 20:553-566.

1448. Weinberg ED: The role of iron in cancer. Eur J Cancer Prev 1996, 5:19-36.

1449. Weinberg ED: Iron loading and disease surveillance. Emerg Infect Dis 1999, 5:346-52.

1450. Núñez MT, Tapia V, Toyokuni S and Okada S: Iron-induced oxidative damage in colon carcinoma (Caco-2) cells. Free Radic Res 200I, 34:57-68.

145I. Glei M, Latunde-Dada GO, Klinder A, Becker TW, Hermann U, Voigt $\mathrm{K}$ and Pool-Zobel BL: Iron-overload induces oxidative DNA damage in the human colon carcinoma cell line HT29 clone 19A. Mutat Res 2002, 519:|5|-61.

1452. Toyokuni S: Iron and carcinogenesis: from Fenton reaction to target genes. Redox Rep 2002, 7:189-97.

1453. Deugnier Y: Iron and liver cancer. Alcohol 2003, 30:|45-150. 
1454. Klaunig JE and Kamendulis LM: The role of oxidative stress in carcinogenesis. Annu Rev Pharmacol Toxicol 2004, 44:239-67.

1455. Storz P: Reactive oxygen species in tumor progression. Front Biosci 2005, 10:1881-96.

|456. Lee SK, Lee J], Lee HJ, Lee J, Jeon BH, Jun CD, Lee SK and Kim EC: Iron chelator-induced growth arrest and cytochrome cdependent apoptosis in immortalized and malignant oral keratinocytes. Journal of Oral Pathology \& Medicine 2006, 35:218-226.

1457. Valko M, Rhodes CJ, Moncol J, Izakovic M and Mazur M: Free radicals, metals and antioxidants in oxidative stressinduced cancer. Chemico-Biological Interactions 2006, I 60: |-40.

I458. Wu J, Eckard J, Chen H, Costa M, Frenkel K and Huang X: Altered iron homeostasis involvement in arsenite-mediated cell transformation. Free Radic Biol Med 2006, 40:444-52.

1459. Kallianpur AR, Lee SA, Gao YT, Lu W, Zheng Y, Ruan ZX, Dai Q, Gu K, Shu XO and Zheng W: Dietary animal-derived iron and fat intake and breast cancer risk in the Shanghai Breast Cancer Study. Breast Cancer Res Treat 2008, 107:123-32.

1460. Prá D, Franke SI, Giulian R, Yoneama ML, Dias JF, Erdtmann B and Henriques JA: Genotoxicity and mutagenicity of iron and copper in mice. Biometals 2008, 21:289-97.

146I. Yang WS and Stockwell BR: Synthetic lethal screening identifies compounds activating iron-dependent, nonapoptotic cell death in oncogenic-RAS-harboring cancer cells. Chem Biol 2008, 15:234-45.

1462. Richardson DR, Kalinowski DS, Lau S, Jansson PJ and Lovejoy DB: Cancer cell iron metabolism and the development of potent iron chelators as anti-tumour agents. Biochim Biophys Acta 2008 in press.

1463. Weitzman SA and Graceffa P: Asbestos catalyzes hydroxyl and superoxide radical generation from hydrogen peroxide. Arch Biochem Biophys 1984, 228:373-6.

1464. Gulumian $M$ and van Wyk JA: Hydroxyl radical production in the presence of fibres by a Fenton-type reaction. Chem Biol Interact 1987, 62:89-97.

I465. Mossman BT, Marsh JP, Shatos MA, Doherty J, Gilbert R and Hill S: Implication of active oxygen species as second messengers of asbestos toxicity. Drug Chem Toxicol 1987, I0:I57-80.

I466. Shatos MA, Doherty JM, Marsh JP and Mossman BT: Prevention of asbestos-induced cell death in rat lung fibroblasts and alveolar macrophages by scavengers of active oxygen species. Environ Res 1987, 44:103-16.

1467. Kamp DW, Graceffa P, Pryor WA and Weitzman SA: The role of free radicals in asbestos-induced diseases. Free Radic Biol Med 1992, I 2:293-315.

1468. Quinlan TR, Marsh JP, Janssen YM, Borm PA and Mossman BT: Oxygen radicals and asbestos-mediated disease. Environ Health Perspect 1994, I02(SuppI I0): I07-I0.

1469. Hardy JA and Aust AE: Iron in asbestos chemistry and carcinogenicity. Chem Rev 1995, 95:97-II8.

1470. Gilmour PS, Brown DM, Beswick PH, MacNee W, Rahman I and Donaldson K: Free radical activity of industrial fibers: role of iron in oxidative stress and activation of transcription factors. Environ Health Perspect 1997, 105(Suppl 5): I3 |3-7.

|47I. Governa M, Amati M, Fontana S, Visona I, Botta GC, Mollo F Bellis D and Bo P: Role of iron in asbestos-body-induced oxidant radical generation. I Toxicol Environ Health A 1999 , 58:279-87.

1472. Kamp DW and Weitzman SA: The molecular basis of asbestos induced lung injury. Thorax 1999, 54:638-52.

1473. Xu A, Wu LJ, Santella RM and Hei TK: Role of oxyradicals in mutagenicity and DNA damage induced by crocidolite asbestos in mammalian cells. Cancer Res 1999, 59:5922-6.

1474. Aljandali A, Pollack H, Yeldandi A, Li Y, Weitzman SA and Kamp DW: Asbestos causes apoptosis in alveolar epithelial cells: role of iron-induced free radicals. J Lab Clin Med 200I, 137:330-9.

1475. Gazzano E, Turci F, Foresti E, Putzu MG, Aldieri E, Silvagno F, Lesci IG, Tomatis M, Riganti C, Romano C, Fubini B, Roveri N and Ghigo D: Iron-loaded synthetic chrysotile: a new model solid for studying the role of iron in asbestos toxicity. Chem Res Toxicol 2007, 20:380-7.

1476. Pervaiz S: Pro-oxidant milieu blunts scissors: Insight into tumor progression, drug resistance, and novel druggable targets. Curr Pharmaceut Des 2006, I 2:4469-4477.

1477. Perrone GG, Tan SX and Dawes IW: Reactive oxygen species and yeast apoptosis. Biochim Biophys Acta 2008, 1783:1354-68.

1478. Scheibel LW and Adler A: Anti-malarial activity of selected aromatic chelators. Mol Pharmacol 1980, I 8:320-325.
1479. Scheibel LW and Rodriguez S: Antimalarial activity of selected aromatic chelators V. Localization of $59 \mathrm{Fe}$ in Plasmodium falciparum in the presence of oxines. Prog Clin Biol Res 1989, 313:1 19-49.

1480. Hershko C Gordeuk VR, Thuma PE, Theanacho EN, Spira DT, Hider RC, Peto TEA and Brittenham GM: The antimalaria effect of iron chelators - studies in animal models and in humans with mild Falciparum malaria. J Inorg Biochem 1992, 47:267-277.

148I. Gordeuk V, Thuma P, Brittenham G, McLaren C, Parry D, Backenstose A, Biemba G, Msiska R, Holmes L, McKinley E, Vargas L, Gilkeson R and Poltera AA: Effect of iron chelation therapy on recovery from deep coma in children with cerebral malaria. New Engl J Med 1992, 327:|473-I477.

1482. Gordeuk VR, Thuma PE, Brittenham GM, Biemba G, Zulu S, Simwanza G, Kalense P, Mhango A, Parry D, Poltera AA and Aikawa M: Iron chelation as a chemotherapeutic strategy for Falciparum malaria. Amer J Trop Med Hyg 1993, 48:193-197.

1483. Voest EE, Vreugdenhil G and Marx JJM: Iron-chelating agents in non-iron overload conditions. Ann Internal Med 1994 , I 20:490-499.

1484. Hider RC and Liu ZD: The treatment of malaria with iron chelators. J Pharm Pharmacol 1997, 49:59-64.

1485. Cabantchik ZI, Moody-Haupt S and Gordeuk VR: Iron chelators as anti-infectives; malaria as a paradigm. FEMS Immunol Med Microbiol 1999, 26:289-98.

1486. Mabeza GF, Loyevsky M, Gordeuk VR and Weiss G: Iron chelation therapy for malaria: A review. Pharmacology \& Therapeutics 1999, 81:53-75.

1487. Fritsche G, Larcher C, Schennach H and Weiss G: Regulatory interactions between iron and nitric oxide metabolism for immune defense against Plasmodium falciparum infection. J Infect Dis 2001, I83:1388-94.

1488. Pradines B, Rolain JM, Ramiandrasoa F, Fusai T, Mosnier J, Rogier C, Daries W, Baret E, Kunesch G, Le Bras J and Parzy D: Iron chelators as antimalarial agents: in vitro activity of dicatecholate against Plasmodium falciparum. J Antimicrob Chemother 2002, 50:177-87.

1489. Birch N, Wang $X$ and Chong HS: Iron chelators as therapeutic iron depletion agents. Exp Opin Therapeut Pat 2006 , 16: I533-1556.

1490. Golenser J, Domb A, Mordechai-Daniel T, Leshem B, Luty A and Kremsner P: Iron chelators: correlation between effects on Plasmodium spp. and immune functions. J Parasitol 2006, 92: $170-7$

|49I. Melnyk P, Leroux V, Sergheraert C and Grellier P: Design, synthesis and in vitro antimalarial activity of an acylhydrazone library. Bioorg Med Chem Lett 2006, 16:31-35.

1492. Pradines B, Tall A, Ramiandrasoa FR, Spiegel A, Sokhna C, Fusai T, Mosnier J, Daries W, Trape JF, Kunesch G, Parzy D and Rogier C: In vitro activity of iron-binding compounds against Senegalese isolates of Plasmodium falciparum. I Antimicrobial Chemotherapy 2006, 57:1093-1099.

1493. Doherty CP: Host-pathogen interactions: the role of iron. J Nutr 2007, 137:1341-4.

1494. Vangapandu S, Jain M, Kaur K, Patil P, Patel SR and Jain R: Recent advances in antimalarial drug development. Medicinal Research Reviews 2007, 27:65-107.

1495. Thuma PE, Mabeza GF, Biemba G, Bhat GJ, McLaren CE, Moyo VM, Zulu S, Khumalo H, Mabeza P, M'Hango A, Parry D, Poltera AA, Brittenham GM and Gordeuk VR: Effect of iron chelation therapy on mortality in Zambian children with cerebral malaria. Transactions of the Royal Society of Tropical Medicine and Hygiene 1998, 92:214-218.

1496. Meshnick SR, Yang YZ, Lima V, Kuypers F, Kamchonwongpaisan S and Yuthavong $Y$ : Iron-dependent free radical generation from the antimalarial agent artemisinin (qinghaosu). Antimicrob Agents Chemother 1993, 37:1 I08-14.

1497. Kamchonwongpaisan S and Meshnick SR: The mode of action of the antimalarial artemisinin and its derivatives. Gen Pharmacol 1996, 27:587-92.

1498. Meshnick SR, Taylor TE and Kamchonwongpaisan S: Artemisinin and the antimalarial endoperoxides: from herbal remedy to targeted chemotherapy. Microbiol Rev 1996, 60:30I-I5.

1499. Wu YK, Yue ZY and Wu YL: Interaction of Qinghaosu (artemisinin) with cysteine sulfhydryl mediated by traces of non-heme iron. Angewandte Chemie-International Edition 1999 , 38:2580-2582. 
1500. Li W, Mo W, Shen D, Sun L, Wang J, Lu S, Gitschier JM and Zhou B: Yeast model uncovers dual roles of mitochondria in action of artemisinin. PLoS Genet 2005, I:e36.

I50 I. Efferth T, Benakis A, Romero MR, Tomicic M, Rauh R, Steinbach D, Hafer R, Stamminger T, Oesch F, Kaina B and Marschall M: Enhancement of cytotoxicity of artemisinins toward cancer cells by ferrous iron. Free Radic Biol Med 2004, 37:998-1009.

1502. Haynes RK, Chan WC, Lung CM, Uhlemann AC, Eckstein U Taramelli D, Parapini S, Monti D and Krishna S: The $\mathbf{F e}^{2+}$. mediated decomposition, PfATP6 Binding, and antimalarial activities of artemisone and other artemisinins: the unlikelihood of $\mathrm{C}$-centered radicals as bioactive intermediates. Chem Med Chem 2007, 2:|480-1497.

I503. Nakase I, Lai H, Singh NP and Sasaki T: Anticancer properties of artemisinin derivatives and their targeted delivery by transferrin conjugation. Int I Pharm 2008, 354:28-33.

1504. White NJ: Qinghaosu (artemisinin): the price of success. Science 2008, 320:330-4.

1505. Eckstein-Ludwig U, Webb RJ, Van Goethem ID, East JM, Lee AG, Kimura M, O'Neill PM, Bray PG, Ward SA and Krishna S: Artemisinins target the SERCA of Plasmodium falciparum. Nature 2003, 424:957-6I

1506. Boyd PW, Jickells T, Law CS, Blain S, Boyle EA, Buesseler KO, Coale KH, Cullen J, de Baar HJ, Follows M, Harvey M, Lancelot C, Levasseur M, Owens NP, Pollard R, Rivkin RB, Sarmiento J, Schoemann V, Smetacek V, Takeda S, Tsuda A, Turner S and Watson AJ: Mesoscale iron enrichment experiments I9932005: synthesis and future directions. Science 2007, 3 15:612-7.

1507. Blain S, Queguiner B, Armand L, Belviso S, Bombled B, Bopp L, Bowie A, Brunet C, Brussaard C, Carlotti F, Christaki U, Corbiere A, Durand I, Ebersbach F, Fuda JL, Garcia N, Gerringa L, Griffiths B, Guigue C, Guillerm C, Jacquet S, Jeandel C, Laan P, Lefevre D, Lo Monaco C, Malits A, Mosseri J, Obernosterer I, Park YH, Picheral M, Pondaven P, Remenyi T, Sandroni V, Sarthou G, Savoye N, Scouarnec L, Souhaut M, Thuiller D, Timmermans K, Trull T, Uitz J van Beek P, Veldhuis $M$, Vincent D, Viollier $E$, Vong $L$ and Wagener T: Effect of natural iron fertilization on carbon sequestration in the Southern Ocean. Nature 2007, 446: 1070-4.

1508. Boyd PW: Biogeochemistry: iron findings. Nature 2007, 446:989-91.

1509. Watson AJ, Bakker DC, Ridgwell AJ, Boyd PW and Law CS: Effect of iron supply on Southern Ocean CO2 uptake and implications for glacial atmospheric CO2. Nature 2000, 407:730-3

1510. Collins HL: Withholding iron as a cellular defence mechanism-friend or foe? Eur J Immunol 2008, 38:1803-6.

I5II. Weinberg ED: Iron and susceptibility to infectious disease. Science 1974, 1 84:952-6.

1512. Weinberg ED: Iron and infection. Microbiol Rev 1978, 42:45-66.

I5I3. Hershko C, Peto TEA and Weatherall DJ: Iron and Infection. British Medical Journal 1988, 296:660-664.

15|4. Oppenheimer SJ: Iron and infection: the clinical evidence. Acta Paediatr Scand Suppl 1989, 36 1:53-62.

I5I5. Otto BR, Verweij vanVught $A$ and Maclaren DM: Transferrins and heme compounds as iron sources for pathogenic bacteria. Critical Reviews in Microbiology 1992, 18:217-233.

1516. Vasil ML and Ochsner UA: The response of Pseudomonas aeruginosa to iron: genetics, biochemistry and virulence. Mol Microbiol 1999, 34:399-413.

I5I7. Bullen J, Griffiths E, Rogers H and Ward G: Sepsis: the critical role of iron. Microbes Infect 2000, 2:409-15

1518. Law D: Virulence factors of Escherichia coli OI57 and other Shiga toxin-producing E. coli. J Appl Microbiol 2000, 88:729-45.

I519. van Asbeck BS, Georgiou NA, Bruggen van der T, Oudshoorn M, Nottet HS and Marx J: Anti-HIV effect of iron chelators: different mechanisms involved. I Clin Virol 200I, 20:|4|-7.

1520. Gordeuk VR, Delanghe JR, Langlois MR and Boelaert JR: Iron status and the outcome of HIV infection: an overview. J Clin Virol 200I, 20: III-5.

I52I. Rodriguez GM and Smith I: Mechanisms of iron regulation in mycobacteria: role in physiology and virulence. Mol Microbiol 2003, 47:|485-94

1522. Bullen JJ, Rogers HJ, Spalding PB and Ward CG: Iron and infection: the heart of the matter. FEMS Immunol Med Microbiol 2005, 43:325-30.

1523. Bullen J], Rogers HJ, Spalding PB and Ward CG: Natural resistance, iron and infection: a challenge for clinical medicine.J Med Microbiol 2006, 55:25I-8.
1524. Maresso AW and Schneewind O: Iron acquisition and transport in Staphylococcus aureus. Biometals 2006, 19:193-203.

1525. McDermid JM and Prentice AM: Iron and infection: effects of host iron status and the iron-regulatory genes haptoglobin and NRAMPI (SLCI|AI) on host-pathogen interactions in tuberculosis and HIV. Clin Sci (Lond) 2006, I I 0:503-24.

1526. Meyer D: Iron chelation as therapy for HIV and Mycobacterium tuberculosis co-infection under conditions of iron overload. Curr Pharm Des 2006, I 2:1943-7.

I527. Sritharan M: Iron and bacterial virulence. Indian J Med Microbiol 2006, 24:163-4.

1528. Boelaert JR, Vandecasteele SJ, Appelberg R and Gordeuk VR: The effect of the host's iron status on tuberculosis. J Infect Dis 2007, I 95:1745-53

1529. Maynor L and Brophy DF: Risk of infection with intravenous iron therapy. Ann Pharmacother 2007, 4I:|476-80.

1530. Basaraba RJ, Bielefeldt-Ohmann H, Eschelbach EK, Reisenhauer C, Tolnay AE, Taraba LC, Shanley CA, Smith EA, Bedwell CL, Chlipala EA and Orme IM: Increased expression of host ironbinding proteins precedes iron accumulation and calcification of primary lung lesions in experimental tuberculosis in the guinea pig. Tuberculosis (Edinb) 2008, 88:69-79.

|53|. Weinberg ED: Iron availability and infection. Biochim Biophys Acta 2008

1532. Ashrafian H: Hepcidin: the missing link between hemochromatosis and infections. Infect Immun 2003, 7I:6693-700.

1533. Saiga H, Nishimura J, Kuwata $H$, Okuyama M, Matsumoto S, Sato $S$ Matsumoto M, Akira S, Yoshikai Y, Honda K, Yamamoto M and Takeda K: Lipocalin 2-dependent inhibition of mycobacteria growth in alveolar epithelium. J Immunol 2008, I 8 I:852 I-7.

1534. Breidbach T, Scory S, Krauth-Siegel RL and Steverding D: Growth inhibition of bloodstream forms of Trypanosoma brucei by the iron chelator deferoxamine. Int J Parasitol 2002, 32:473-479.

I535. Merschjohann $\mathrm{K}$ and Steverding D: In vitro growth inhibition of bloodstream forms of Trypanosoma brucei and Trypanosoma congolense by iron chelators. Kinetoplastid Biol Dis 2006, 5:3.

1536. Drakesmith $H$ and Prentice $A$ : Viral infection and iron metabolism. Nat Rev Microbiol 2008, 6:54l-52.

1537. Kaprelyants AS, Gottschal JC and Kell DB: Dormancy in nonsporulating bacteria. FEMS Microbiol Rev 1993, I 0:27I-286.

1538. Kell DB, Kaprelyants AS, Weichart DH, Harwood $\mathrm{CL}$ and Barer MR: Viability and activity in readily culturable bacteria: a review and discussion of the practical issues. Antonie van Leeuwenhoek 1998, 73:169-187.

1539. Goris RJ: Mediators of multiple organ failure. Intensive Care Med 1990, I6(Suppl 3):SI92-6.

1540. Suntharalingam G, Perry MR, Ward S, Brett SJ, Castello-Cortes A Brunner MD and Panoskaltsis N: Cytokine storm in a phase I trial of the anti-CD28 monoclonal antibody TGN14I2. N Engl J Med 2006, 355:1018-28.

154I. Beal AL and Cerra FB: Multiple Organ Failure Syndrome in the 1990s - Systemic Inflammatory Response and Organ Dysfunction. Jama-Journal of the American Medical Association 1994, 271:226-233.

1542. Bolton CF: Sepsis and the systemic inflammatory response syndrome: Neuromuscular manifestations. Critical Care Medicine 1996, 24:|408-1416.

1543. Bone RC: Toward a theory regarding the pathogenesis of the systemic inflammatory response syndrome: What we do and do not know about cytokine regulation. Critical Care Medicine 1996, 24:163-172.

1544. Davies MG and Hagen PO: Systemic inflammatory response syndrome. British Journal of Surgery 1997, 84:920-935.

1545. Baue $A E$, Durham $R$ and Faist $E$ : Systemic inflammatory response syndrome (SIRS), multiple organ dysfunction syndrome (MODS), multiple organ failure (MOF): Are we winning the battle? Shock 1998, 10:79-89.

1546. Gutteridge JMC and Mitchell J: Redox imbalance in the critically ill. British Medical Bulletin 1999, 55:49-75

1547. Johnson D and Mayers I: Multiple organ dysfunction syndrome: a narrative review. Can J Anaesth 2001, 48:502-9.

1548. Martin GS, Mannino DM, Eaton S and Moss M: The epidemiology of sepsis in the United States from 1979 through 2000. New England Journal of Medicine 2003, 348:I546-I554.

1549. Keel $M$ and Trentz O: Pathophysiology of polytrauma. Injury $2005,36: 691-709$.

1550. Nguyen HB, Rivers EP, Abrahamian FM, Moran G], Abraham E, Trzeciak S, Huang DT, Osborn T, Stevens D and Talan DA: Severe 
sepsis and septic shock: Review of the literature and emergency department management guidelines. Ann Emergency Med 2006, 48:28-54.

155I. Protti A and Singer M: Bench-to-bedside review: Potential strategies to protect or reverse mitochondrial dysfunction in sepsis-induced organ failure. Critical Care 2006, I0:227-233.

1552. Papathanassoglou EDE, Moynihan JA and Ackerman MH: Does programmed cell death (apoptosis) play a role in the development of multiple organ dysfunction in critically ill patients? A review and a theoretical framework. Critical Care Medicine 2000, 28:537-549.

1553. Festjens N, Berghe Vanden T and Vandenabeele P: Necrosis, a well-orchestrated form of cell demise: Signalling cascades, important mediators and concomitant immune response. Biochim Biophys Acta 2006, I 757:| 37|-1387.

I554. Golstein P and Kroemer G: Cell death by necrosis: towards a molecular definition. Trends in Biochemical Sciences 2007, 32:37-43.

1555. Goode HF and Webster NR: Free radicals and antioxidants in sepsis. Crit Care Med 1993, 2 I:I770-6.

1556. Redl H, Gasser H, Schlag G and Marzi I: Involvement of oxygen radicals in shock related cell injury. $\mathrm{Br}$ Med Bull 1993, 49:556-65

1557. Horn KD: Evolving strategies in the treatment of sepsis and systemic inflammatory response syndrome (SIRS). QJM 1998, 91:265-77.

1558. Bulger EM and Maier RV: Antioxidants in critical illness. Arch Surg 200I, I36:1201-7.

1559. Cadenas $S$ and Cadenas AM: Fighting the stranger-antioxidant protection against endotoxin toxicity. Toxicology 2002 , 180:45-63.

1560. Victor VM, Rocha $M$ and De la Fuente M: Immune cells: free radicals and antioxidants in sepsis. Int Immunopharmacol 2004, 4:327-47.

156I. Victor VM, Rocha M, Esplugues JV and De la Fuente M: Role of free radicals in sepsis: Antioxidant therapy. Current Pharmaceutical Design 2005, I I:3 |4I-3 I58

1562. Vlessis AA, Goldman RK and Trunkey DD: New concepts in the pathophysiology of oxygen metabolism during sepsis. $\mathrm{Br} J$ Surg 1995, 82:870-6.

1563. Quinlan GJ, Chen Y, Evans TW and Gutteridge JMC: Iron signalling regulated directly and through oxygen: implications for sepsis and the acute respiratory distress syndrome. Clinical Science 200 I, I00:169-182.

1564. Closa D and Folch-Puy E: Oxygen free radicals and the systemic inflammatory response. IUBMB Life 2004, 56:185-191.

1565. Abdelrahman M, Mazzon E, Bauer M, Bauer I, Delbosc S, Cristol JP, Patel NS, Cuzzocrea $S$ and Thiemermann C: Inhibitors of NADPH oxidase reduce the organ injury in hemorrhagic shock. Shock 2005, 23:107-|4.

I566. Javadi P, Buchman TG, Stromberg PE, Turnbull IR, Vyas D, Hotchkiss RS, Karl IE and Coopersmith CM: Iron dysregulation combined with aging prevents sepsis-induced apoptosis. J Surg Res 2005, I 28:37-44.

1567. Crimi E, Sica V, Slutsky AS, Zhang HB, Williams-Ignarro S, Ignarro LJ and Napoli C: Role of oxidative stress in experimental sepsis and multisystem organ dysfunction. Free Radical Research 2006, 40:665-672.

1568. Crimi E, Sica V, Williams-Ignarro S, Zhang H, Slutsky AS, Ignarro L and Napoli C: The role of oxidative stress in adult critica care. Free Radic Biol Med 2006, 40:398-406.

1569. Lemineur T, Deby-Dupont G and Preiser JC: Biomarkers of oxidative stress in critically ill patients: what should be measured, when and how? Curr Op Clin Nutr Metab Care 2006, 9:704-710.

1570. Lagan AL, Melley DD, Evans TW and Quinlan G]: Pathogenesis of the systemic inflammatory syndrome and acute lung injury: role of iron mobilization and decompartmentalization. Am J Physiol Lung Cell Mol Physiol 2008, 294:LI6I-74.

157I. Galley HF and Webster NR: Elevated serum bleomycindetectable iron concentrations in patients with sepsis syndrome. Intensive Care Med 1996, 22:226-9.

1572. Galley HF, Davies MJ and Webster NR: Ascorbyl radical formation in patients with sepsis: effect of ascorbate loading. Free Radic Biol Med 1996, 20:139-43.

1573. Galley HF, Howdle PD, Walker BE and Webster NR: The effects of intravenous antioxidants in patients with septic shock. Free Radic Biol Med 1997, 23:768-74.
1574. Eaton JW and Qian MW: Molecular bases of cellular iron toxicity. Free Radical Biology and Medicine 2002, 32:833-840.

1575. Yu Z, Persson HL, Eaton JW and Brunk UT: Intralysosomal iron: a major determinant of oxidant-induced cell death. Free Radic Biol Med 2003, 34:1243-52.

1576. Guicciardi ME, Leist $M$ and Gores GJ: Lysosomes in cell death. Oncogene 2004, 23:288I-90.

1577. Terman A, Kurz T, Gustafsson B and Brunk UT: Lysosomal labilization. IUBMB Life 2006, 58:53I-9.

1578. Vulcano M, Meiss RP and Isturiz MA: Deferoxamine reduces tissue injury and lethality in LPS-treated mice. Int Immunopharmacol 2000, 22:635-44.

1579. Cuzzocrea S, Rossi A, Pisano B, Di Paola R, Genovese T, Patel NS, Cuzzocrea E, lanaro A, Sautebin L, Fulia F, Chatterjee PK, Caputi AP and Thiemermann C: Pyrrolidine dithiocarbamate attenuates the development of organ failure induced by zymosan in mice. Intensive Care Med 2003, 29:2016-25.

I 580. Persson HL, Yu Z, Tirosh O, Eaton JW and Brunk UT: Prevention of oxidant-induced cell death by lysosomotropic iron chelators. Free Radic Biol Med 2003, 34:1295-305.

158I. Messaris E, Antonakis PT, Memos N, Chatzigianni E, Leandros E and Konstadoulakis MM: Deferoxamine administration in septic animals: improved survival and altered apoptotic gene expression. Int Immunopharmacol 2004, 4:455-9.

1582. Tracey KJ and Cerami A: Tumor Necrosis Factor: a pleiotropic cytokine and therapeutic target. Annu Rev Med I 994, 45:491-503.

1583. Opal SM and Huber CE: Bench-to-bedside review: Toll-like receptors and their role in septic shock. Critical Care 2002, 6:125-136.

1584. Hopkins $\mathrm{P}$ and Cohen J: Toll-like receptors: the key to the stable door? Crit Care 2002, 6:99-101.

1585. Reynolds FD, Dauchy R, Blask D, Dietz PA, Lynch D and Zuckerman R: The pineal gland hormone melatonin improves survival in a rat model of sepsis/shock induced by zymosan A. Surgery 2003, I 34:474-479.

1586. Carrillo-Vico A, Lardone PJ, Naji L, Fernández-Santos JM, MartínLacave I, Guerrero JM and Calvo JR: Beneficial pleiotropic actions of melatonin in an experimental model of septic shock in mice: regulation of pro-/anti-inflammatory cytokine network, protection against oxidative damage and anti-apoptotic effects. J Pineal Res 2005, 39:400-408.

1587. Escames G, Acuna-Castroviejo D, López LC, Tan DX, Maldonado MD, Sanchez-Hidalgo M, León J and Reiter RJ: Pharmacological utility of melatonin in the treatment of septic shock: experimental and clinical evidence. J Pharm Pharmacol 2006, 58: I 153-1165.

1588. Cuzzocrea S, Riley DP, Caputi AP and Salvemini D: Antioxidant therapy: a new pharmacological approach in shock, inflammation, and ischemia/reperfusion injury. Pharmacol Rev 2001, 53:135-59.

1589. Nathens AB, Neff MJ, Jurkovich GJ, Klotz P, Farver K, Ruzinski JT, Radella F, Garcia I and Maier RV: Randomized, prospective trial of antioxidant supplementation in critically ill surgical patients. Annals of Surgery 2002, 236:814-822.

1590. Cuzzocrea S, Thiemermann C and Salvemini D: Potential therapeutic effect of antioxidant therapy in shock and inflammation. Current Medicinal Chemistry 2004, I I: ||47-I I62.

159I. Di Paola R, Mazzon E, Muia C, Crisafulli C, Genovese T, Di Bella P, Esposito E, Menegazzi M, Meli R, Suzuki H and Cuzzocrea S: Green tea polyphenol extract attenuates zymosan-induced nonseptic shock in mice. Shock 2006, 26:402-9.

1592. Ritter C, Andrades ME, Reinke A, Menna-Barreto S, Moreira JC and Dal-Pizzol F: Treatment with $\mathbf{N}$-acetylcysteine plus deferoxamine protects rats against oxidative stress and improves survival in sepsis. Crit Care Med 2004, 32:342-9.

1593. Ritter $C$, da Cunha AA, Echer IC, Andrades M, Reinke A Lucchiari N, Rocha J, Streck EL, Menna-Barreto S, Moreira JC and Dal-Pizzol F: Effects of $\mathbf{N}$-acetylcysteine plus deferoxamine in lipopolysaccharide-induced acute lung injury in the rat. Crit Care Med 2006, 34:47I-7.

1594. Pinho RA, Silveira PC, Silva LA, Luiz Streck E, Dal-Pizzol F and IC FM: N-acetylcysteine and deferoxamine reduce pulmonary oxidative stress and inflammation in rats after coal dust exposure. Environ Res 2005, 99:355-60.

1595. Teixeira KC, Soares FS, Rocha LG, Silveira PC, Silva LA, Valenca SS, Pizzol FD, Streck EL and Pinho RA: Attenuation of bleomycininduced lung injury and oxidative stress by $\mathbf{N}$-acetylcysteine plus deferoxamine. Pulm Pharmacol Ther 2008, 21:309-16. 
1596. Abdelrahman M, Sharples EJ, McDonald MC, Collin M, Patel NS, Yaqoob MM and Thiemermann C: Erythropoietin attenuates the tissue injury associated with hemorrhagic shock and myocardial ischemia. Shock 2004, 22:63-9.

1597. Cuzzocrea S, Di Paola R, Mazzon E, Patel NS, Genovese T, Muia C, Crisafulli C, Caputi AP and Thiemermann C: Erythropoietin reduces the development of nonseptic shock induced by zymosan in mice. Crit Care Med 2006, 34: I I 68-77.

1598. Lovitt RW, Kell DB and Morris JG: Proline reduction by Clostridium sporogenes is coupled to vectorial proton ejection. FEMS Microbiology Letters 1986, 36:269-273.

1599. Kell DB and Mendes P: Snapshots of systems: metabolic control analysis and biotechnology in the post-genomic era. Technological and Medical Implications of Metabolic Control Analysis Kluwer Academic Publishers, Dordrecht: Cornish-Bowden A, Cárdenas ML 2000, 3-25 http://dbkgroup.org/WhitePapers/ mcabio.htm.

1600. Wikström MKF: The different cytochrome $\boldsymbol{b}$ components in the respiratory chain of animal mitochondria and their role in electron transport and energy conservation. Biochim Biophys Acta 1973, 301:155-93.

I60I. John P and Papa S: Rapid oxygen-induced reduction of b-type cytochromes in Paracoccus denitrificans. FEBS Lett 1978 85: $179-82$

1602. Reif DW: Ferritin as a source of iron for oxidative damage. Free Rad Biol Med 1992, 12:417-427.

1603. Moison RM, Bloemhof FE, Geerdink JA, de Beaufort AJ and Berger HM: The capacity of different infusion fluids to lower the prooxidant activity of plasma iron: an important factor in resuscitation? Transfusion 2000, 40:|346-5|.

1604. Fábián I and Csordás V: Metal ion catalyzed autoxidation reactions: Kinetics and mechanisms. Adv Inorg Chem 2003, 54:395-46I.

1605. Duarte TL, Almeida GM and Jones GD: Investigation of the role of extracellular $\mathrm{H}_{2} \mathrm{O}_{2}$ and transition metal ions in the genotoxic action of ascorbic acid in cell culture models. Toxicol Lett 2007, 170:57-65.

1606. Wang S, Geraci G, Kuhlmann MK, Levin NW and Handelman GJ: Chemical reactions of vitamin $\mathbf{C}$ with intravenous-iron formulations. Nephrol Dial Transplant 2008, 23:| 20-5.

1607. Yagi K, Ishida N, Komura S, Ohishi N, Kusai $M$ and Kohno $M$ : Generation of hydroxyl radical from linoleic acid hydroperoxide in the presence of epinephrine and iron. Biochem Biophys Res Commun 1992, I 83:945-5I.

1608. Everse $J$ and Hsia N: The toxicities of native and modified hemoglobins. Free Radical Biology and Medicine 1997 22: $1075-1099$.

1609. Medina I, Tombo I, Satue-Gracia MT, German JB and Frankel EN: Effects of natural phenolic compounds on the antioxidant activity of lactoferrin in liposomes and oil-in-water emulsions. J Agric Food Chem 2002, 50:2392-9.

1610. Hoppe $M$, Hulthen $L$ and Hallberg $L:$ The relative bioavailability in humans of elemental iron powders for use in food fortification. Eur J Nutrition 2006, 45:37-44.

16II. Goodell B, Jellison J, Liu J, Daniel G, Paszczynski A, Fekete F, Krishnamurthy $S$, Jun $L$ and $X u G$ : Low molecular weight chelators and phenolic compounds isolated from wood decay fungi and their role in the fungal biodegradation of wood. J Biotechnol 1997, 53:133-162.

1612. Mályusz M, Kahler W and Gronow G: Hippurate metabolism as a hydroxyl radical trapping mechanism in the rat kidney. Kidney Blood Press Res 200I, 24:|49-58.

16|3. Brzezinski A: Melatonin in humans. N Engl J Med I997, 336: 186-95

16|4. Reiter R, Tang L, Garcia JJ and MunozHoyos A: Pharmacological actions of melatonin in oxygen radical pathophysiology. Life Sci 1997, 60:2255-227I.

1615. Beyer CE, Steketee JD and Saphier D: Antioxidant properties of melatonin - An emerging mystery. Biochem Pharmacol 1998, 56: $1265-1272$.

1616. Reiter RJ: Oxidative damage in the central nervous system: protection by melatonin. Progr Neurobiol 1998, 56:359-384.

1617. Cuzzocrea S, Costantino G, Gitto E, Mazzon E, Fulia F, Serraino I, Cordaro S, Barberi I, De Sarro A and Caputi AP: Protective effects of melatonin in ischemic brain injury. J Pineal Res 2000 , 29:217-27.

1618. Karbownik M, Lewinski A and Reiter RJ: Anticarcinogenic actions of melatonin which involve antioxidative processes: comparison with other antioxidants. International Journal of Biochemistry \& Cell Biology 200 I, 33:735-753.
1619. Letechipia-Vallejo G, Gonzalez-Burgos I and Cervantes M: Neuroprotective effect of melatonin on brain damage induced by acute global cerebral ischemia in cats. Arch Med Res 200I, 32: $186-92$.

1620. Okatani Y, Wakatsuki A, Shinohara K, Taniguchi $\mathrm{K}$ and Fukaya $\mathrm{T}$ : Melatonin protects against oxidative mitochondrial damage induced in rat placenta by ischemia and reperfusion. J Pineal Res 2001, 31:173-8.

162I. Reiter RJ, Tan DX, Manchester LC and Qi WB: Biochemical reactivity of melatonin with reactive oxygen and nitrogen species - A review of the evidence. Cell Biochemistry and Biophysics 200I, 34:237-256.

1622. Allegra M, Reiter RJ, Tan DX, Gentile C, Tesoriere L and Livrea MA: The chemistry of melatonin's interaction with reactive species. Journal of Pineal Research 2003, 34: I-10.

1623. Cheung RTF: The utility of melatonin in reducing cerebral damage resulting from ischemia and reperfusion. Journal of Pineal Research 2003, 34:153-160.

1624. Okatani Y, Wakatsuki A, Reiter RJ, Enzan $\mathrm{H}$ and Miyahara $\mathrm{Y}$ : Protective effect of melatonin against mitochondrial injury induced by ischemia and reperfusion of rat liver. Eur Pharmacol 2003, 469:145-I52.

1625. Pei Z, Pang SF and Cheung RTF: Administration of melatonin after onset of ischemia reduces the volume of cerebral infarction in a rat middle cerebral artery occlusion stroke model. Stroke 2003, 34:770-775.

1626. Reiter RJ and Tan DX: Melatonin: a novel protective agent against oxidative injury of the ischemic/reperfused heart. Cardiovascular Research 2003, 58:10-19.

1627. Reiter RJ: Melatonin: clinical relevance. Best Pract Res Clin Endocrinol Metab 2003, I 7:273-85.

1628. Weiss JF and Landauer MR: Protection against ionizing radiation by antioxidant nutrients and phytochemicals. Toxicology 2003, I 89: I-20.

1629. Macleod MR, O'Collins T, Horky LL, Howells DW and Donnan GA: Systematic review and meta-analysis of the efficacy of melatonin in experimental stroke. J Pineal Res 2005, 38:35-4I.

1630. Srinivasan V, Pandi-Perumal SR, Maestroni GJM, Esquifino AI, Hardeland R and Cardinali DP: Role of melatonin in neurodegenerative diseases. Neurotox Res 2005, 7:293-318.

I63I. Hardeland R, Pandi-Perumal SR and Cardinali DP: Melatonin. International Journal of Biochemistry \& Cell Biology 2006, 38:3 I 3-3 I6.

1632. Grootveld $M$ and Halliwell $B$ : Aromatic hydroxylation as a potential measure of hydroxyl-radical formation in vivo. Identification of hydroxylated derivatives of salicylate in human body fluids. Biochem J 1986, 237:499-504.

1633. Roob JM, Khoschsorur G, Tiran A, Horina JH, Holzer $\mathrm{H}$ and Winklhofer-Roob BM: Vitamin E attenuates oxidative stress induced by intravenous iron in patients on hemodialysis. Am Soc Nephrol 2000, I I:539-49.

1634. Oldham KM and Bowen PE: Oxidative stress in critical care: is antioxidant supplementation beneficial? J Am Diet Assoc 1998, 98: $1001-8$.

1635. Rehman A, Collis CS, Yang M, Kelly M, Diplock AT, Halliwell B and Rice-Evans C: The effects of iron and vitamin $C$ cosupplementation on oxidative damage to DNA in healthy volunteers. Biochem Biophys Res Comm 1998, 246:293-298.

1636. Gilgun-Sherki Y, Rosenbaum Z, Melamed E and Offen D: Antioxidant therapy in acute central nervous system injury: current state. Pharmacol Rev 2002, 54:27I-84.

1637. Miller ER 3rd, Pastor-Barriuso R, Dalal D, Riemersma RA, Appel LJ and Guallar E: Meta-analysis: high-dosage vitamin $E$ supplementation may increase all-cause mortality. Ann Intern Med 2005, I 42:37-46.

1638. Rodrigo R, Guichard C and Charles R: Clinical pharmacology and therapeutic use of antioxidant vitamins. Fund Clin Pharmacol 2007, 21: I | |-127.

1639. Bjelakovic G, Nikolova D, Gluud LL, Simonetti RG and Gluud C Mortality in randomized trials of antioxidant supplements for primary and secondary prevention: systematic review and meta-analysis. JAMA 2007, 297:842-57.

1640. Orrell RW, Lane RJ and Ross M: Antioxidant treatment for amyotrophic lateral sclerosis/motor neuron disease. Cochrane Database Syst Rev 2007, CD002829.

164I. Stephens NG, Parsons A, Schofield PM, Kelly F, Cheeseman K and Mitchinson MJ: Randomised controlled trial of vitamin $E$ in patients with coronary disease: Cambridge Heart Antioxidant Study (CHAOS). Lancet 1996, 347:78l-6.

1642. Yusuf S, Dagenais G, Pogue J, Bosch J and Sleight P: Vitamin E supplementation and cardiovascular events in high-risk 
patients. The Heart Outcomes Prevention Evaluation Study Investigators. N Engl | Med 2000, 342:|54-60.

1643. Lee DH, Folsom AR, Harnack L, Halliwell B and Jacobs DR Jr: Does supplemental vitamin $C$ increase cardiovascular disease risk in women with diabetes? Am / Clin Nutr 2004, 80: I I 94-200.

1644. Williams KJ and Fisher EA: Oxidation, lipoproteins, and atherosclerosis: which is wrong, the antioxidants or the theory? Curr Opin Clin Nutr Metab Care 2005, 8:|39-46.

1645. Papaharalambus CA and Griendling KK: Basic mechanisms of oxidative stress and reactive oxygen species in cardiovascular injury. Trends Cardiovasc Med 2007, I 7:48-54.

1646. O'Collins VE, Macleod MR, Donnan GA, Horky LL, Worp van de $\mathrm{BH}$ and Howells DW: I,026 experimental treatments in acute stroke. Ann Neurol 2006, 59:467-77.

647. Evans JR and Henshaw K: Antioxidant vitamin and minera supplements for preventing age-related macular degeneration. Cochrane Database Syst Rev 2008, CD000253.

1648. Jeyabalan A and Caritis SN: Antioxidants and the prevention of preeclampsia - Unresolved issues. N Engl J Med 2006 , 354: $184 \mid-1843$

1649. Poston L, Briley AL, Seed PT, Kelly FJ and Shennan AH: Vitamin C and vitamin $E$ in pregnant women at risk for pre-eclampsia (VIP trial): randomised placebo-controlled trial. Lancet 2006 367: I| $45-54$.

1650. Rumbold AR, Crowther CA, Haslam RR, Dekker GA and Robinson JS: Vitamins $\mathbf{C}$ and $\mathbf{E}$ and the risks of preeclampsia and perinatal complications. N Engl J Med 2006, 354:1796-806.

165I. Spinnato JA: New therapies in the prevention of preeclampsia. Current Opinion in Obstetrics \& Gynecology 2006, | 8:60 |-604.

1652. Rumbold A, Duley L, Crowther CA and Haslam RR: Antioxidants for preventing pre-eclampsia. Cochrane Database Syst Rev 2008, CD004227

1653. Coleman NA: Antioxidants in critical care medicine. Env Toxicol Pharmacol 2001, 10:183-188

1654. Bayne AC and Sohal RS: Effects of superoxide dismutase catalase mimetics on life span and oxidative stress resistance in the housefly, Musca domestica. Free Radic Bio Med 2002, 32:1229-34.

1655. Magwere T, West M, Riyahi K, Murphy MP, Smith RA and Partridge L: The effects of exogenous antioxidants on lifespan and oxidative stress resistance in Drosophila melanogaster. Mech Ageing Dev 2006, I 27:356-70.

1656. Omenn GS, Goodman GE, Thornquist MD, Balmes I, Cullen MR Glass A, Keogh JP, Meyskens FL, Valanis B, Williams JH, Barnhart S and Hammar S: Effects of a combination of beta carotene and vitamin $\mathbf{A}$ on lung cancer and cardiovascular disease. $N$ Engl J Med 1996, 334: II50-5.

1657. Bailey DM, Raman S, McEneny J, Young IS, Parham KL, Hullin DA Davies B, McKeeman G, McCord JM and Lewis MH: Vitamin C prophylaxis promotes oxidative lipid damage during surgical ischemia-reperfusion. Free Radical Biology and Medicine 2006 40:591-600

1658. Bjelakovic G, Nikolova D, Gluud LL, Simonetti RG and Gluud C: Antioxidant supplements for prevention of mortality in healthy participants and patients with various diseases. Cochrane Database of Systematic Reviews 2008 2008, CD007I 76.

1659. Long LH, Clement MV and Halliwell B: Artifacts in cell culture: Rapid generation of hydrogen peroxide on addition of (-) epigallocatechin, (-)-epigallocatechin gallate, (+)-catechin, and quercetin to commonly used cell culture media. Biochem Biophys Res Comm 2000, 273:50-53.

I660. Hininger I, Waters R, Osman M, Garrel C, Fernholz K, Roussel AM and Anderson RA: Acute prooxidant effects of vitamin $C$ in EDTA chelation therapy and long-term antioxidant benefits of therapy. Free Rad Biol Med 2005, 38:1565-1570.

I66I. Szewczyk A and Wojtczak L: Mitochondria as a pharmacological target. Pharmacol Rev 2002, 54:101-27.

1662. Murphy MP and Smith RA: Targeting antioxidants to mitochondria by conjugation to lipophilic cations. Annu Rev Pharmacol Toxicol 2007, 47:629-56.

1663. Jou MJ: Pathophysiological and pharmacological implications of mitochondria-targeted reactive oxygen species generation in astrocytes. Adv Drug Deliv Rev 2008, 60:1512-26.

1664. Balcerczyk A, Sowa K and Bartou G: Metal chelators react also with reactive oxygen and nitrogen species. Biochem Biophys Res Comm 2007, 352:522-525.

1665. Hider RC and Zhou T: The design of orally active iron chelators. Ann N Y Acad Sci 2005, 1054:14|-54.

1666. Marcus RA: Theory of Oxidation-Reduction Reactions Involving Electron Transfer .I.J Chem Phys 1956, 24:966-978.
1667. Marcus RA: Chemical + Electrochemical Electron-Transfer Theory. Annu Rev Phys Chem 1964, I 5: I55.

1668. Chou M, Creutz C and Sutin N: Rate constants and activation parameters for outer-sphere electron transfer reactions and comparisons with predictions of Marcus theory. JACS 1977, 99:5615-5623.

1669. Marcus RA and Sutin N: Electron transfers in chemistry and biology. Biochim Biophys Acta 1985, 8I I:265-322.

1670. Liu ZD and Hider RC: Design of iron chelators with therapeutic application. Coord Chem Rev 2002, 232:|5|-|7|.

167I. Liu ZD and Hider RC: Design of clinically useful iron(III)selective chelators. Medicinal Res Rev 2002, 22:26-64.

1672. Richardson DR and Ponka P: Development of iron chelators to treat iron overload disease and their use as experimenta tools to probe intracellular iron metabolism. Am J Hemato 1998, 58:299-305.

1673. Aouad F, Florence A, Zhang Y, Collins F, Henry C, Ward RJ and Crichton RR: Evaluation of new iron chelators and their therapeutic potential. Inorganica Chimica Acta 2002, 339:470-480

1674. Bush Al: Metal complexing agents as therapies for Alzheimer's disease. Neurobiology of Aging 2002, 23:1031-1038.

1675. Crichton RR, Florence $A$ and Ward RJ: Aluminium and iron in the brain - prospects for chelation. Coordination Chemistry Reviews 2002, 228:365-37।.

1676. Chaston TB and Richardson DR: Iron chelators for the treatment of iron overload disease: relationship between structure, redox activity, and toxicity. Amer / Hematol 2003, 73:200-210

1677. Crichton RR and Ward RJ: Iron chelators and their therapeutic potential. Metal lons in Biological Systems, Metal lons and Their Complexes in Medication, Metal lons in Biological Systems 2004, 41:185-219.

1678. Bernhardt PV: Coordination chemistry and biology of chelators for the treatment of iron overload disorders. Dalton Trans 2007, 3214-3220.

1679. Leeson PD and Springthorpe B: The influence of drug-like concepts on decision-making in medicinal chemistry. Nat Rev Drug Discov 2007, 6:88I-90.

1680. Imbert $M$, Bechet $M$ and Blondeau R: Comparison of the Main Siderophores Produced by Some Species of Streptomyces. Current Microbiology 1995, 31:I29-I33.

I68I. Flores FJ, Rincón J and Martín JF: Characterization of the ironregulated desA promoter of Streptomyces pilosus as a system for controlled gene expression in actinomycetes. Microb Cell Fact 2003, 2:5.

1682. Catnach SM, Fairclough PD and Hammond SM: Intestinal absorption of peptide drugs: advances in our understanding and clinical implications. Gut 1994, 35:44I-4.

1683. Bailey PD, Boyd CA, Bronk JR, Collier ID, Meredith D, Morgan KM and Temple CS: How to make drugs orally active: a substrate template for peptide transporter PepTI. Angew Chem Int Ed Engl 2000, 39:505-508.

1684. Terada $\mathrm{T}$ and Inui $\mathrm{K}$ : Peptide transporters: structure, function, regulation and application for drug delivery. Curr Drug Metab 2004, 5:85-94.

1685. Bailey PD, Boyd CA, Collier ID, George JG, Kellett GL, Meredith D, Morgan KM, Pettecrew R, Price RA and Pritchard RG: Conformational and spacial preferences for substrates of PepTI. Chem Commun (Camb) 2005, 5352-4.

1686. Maggio A: Light and shadows in the iron chelation treatment of haematological diseases. Br J Haematol 2007, I38:407-2 I.

1687. Kontoghiorghes G], Neocleous K and Kolnagou A: Benefits and risks of deferiprone in iron overload in Thalassaemia and other conditions: comparison of epidemiological and therapeutic aspects with deferoxamine. Drug Saf 2003 26:553-84.

1688. Anderson LJ, Wonke B, Prescott E, Holden S, Walker JM and Pennell DJ: Comparison of effects of oral deferiprone and subcutaneous desferrioxamine on myocardial iron concentrations and ventricular function in beta-thalassaemia. Lancet 2002, 360:516-20.

1689. Heinz U, Hegetschweiler K, Acklin P, Faller B, Lattmann R and Schnebli HP: 4-[3,5-Bis(2-hydroxyphenyl)-I,2,4-triazol-I-yl]benzoic acid: a novel efficient and selective iron(III) complexing agent. Angew Chem Int Ed Engl 1999, 38:2568-2570.

1690. Nick H, Acklin P, Lattmann R, Buehlmayer P, Hauffe S, Schupp J and Alberti D: Development of tridentate iron chelators: From desferrithiocin to ICL670. Curr Med Chem 2003, I0:1065-1076. 
169I. Nisbet-Brown E, Olivieri NF, Giardina PJ, Grady RW, Neufeld EJ, Sechaud R, Krebs-Brown AJ, Anderson JR, Alberti D, Sizer KC and Nathan DG: Effectiveness and safety of ICL670 in ironloaded patients with thalassaemia: a randomised, doubleblind, placebo-controlled, dose-escalation trial. Lancet 2003, 36 I: 1597-602

1692. Kontoghiorghes G]: Effects of ICL670 (deferasirox) on cardiac iron concentrations. Lancet 2005, 366:804.

1693. Cappellini MD, Cohen A, Piga A, Bejaoui M, Perrotta S, Agaoglu L, Aydinok Y, Kattamis A, Kilinc Y, Porter J, Capra M, Galanello R, Fattoum S, Drelichman G, Magnano C, Verissimo M, AthanassiouMetaxa M, Giardina P, Kourakli-Symeonidis A, Janka-Schaub G, Coates T, Vermylen C, Olivieri N, Thuret I, Opitz H, RessayreDjaffer C, Marks P and Alberti D: A phase 3 study of deferasirox (ICL670), a once-daily oral iron chelator, in patients with beta-thalassemia. Blood 2006, 107:3455-62.

1694. Chantrel-Groussard K, Gaboriau F, Pasdeloup N, Havouis R, Nick H, Pierre JL, Brissot $P$ and Lescoat $G$ : The new orally active iron chelator ICL670A exhibits a higher antiproliferative effect in human hepatocyte cultures than O-trensox. Eur J Pharmacol 2006, 541:129-137.

1695. Glickstein H, El RB, Link G, Breuer W, Konijn AM, Hershko C, Nick $\mathrm{H}$ and Cabantchik $\mathrm{Zl}$ : Action of chelators in iron-loaded cardiac cells: Accessibility to intracellular labile iron and functional consequences. Blood 2006, I08:3195-203.

1696. Kontoghiorghes G]: Future chelation monotherapy and combination therapy strategies in thalassemia and other conditions. comparison of deferiprone deferoxamine, ICL670, GT56-252, LINAII and starch deferoxamine polymers. Hemoglobin 2006, 30:329-47.

1697. Piga A, Galanello R, Forni GL, Cappellini MD, Origa R, Zappu A, Donato G, Bordone E, Lavagetto A, Zanaboni L, Sechaud R, Hewson N, Ford JM, Opitz H and Alberti D: Randomized phase II trial of deferasirox (Exjade, ICL670), a once-daily, orallyadministered iron chelator, in comparison to deferoxamine in thalassemia patients with transfusional iron overload. Haematologica 2006, 91:873-80.

1698. Porter JB: Deferasirox: An effective once-daily orally active iron chelator. Drugs of Today 2006, 42:623-637.

1699. VanOrden HE and Hagemann TM: Deferasirox-an oral agent for chronic iron overload. Ann Pharmacother 2006, 40: II I0-7.

1700. Nick H: Iron chelation, quo vadis? Curr Opin Chem Biol 2007, I I:419-23

I70I. Yang LP, Keam SJ and Keating GM: Deferasirox: a review of its use in the management of transfusional chronic iron overload. Drugs 2007, 67:2211-30.

1702. Vermylen C: What is new in iron overload? Eur J Pediatr 2008 167:377-8I.

1703. Dobson PD, Patel $Y$ and Kell DB: "Metabolite-likeness" as a criterion in the design and selection of pharmaceutical drug libraries. Drug Disc Today 2008 in press.

1704. Borisy AA, Elliott PJ, Hurst NW, Lee MS, Lehar J, Price ER, Serbedzija G, Zimmermann GR, Foley MA, Stockwell BR and Keith CT: Systematic discovery of multicomponent therapeutics. Proc Natl Acad Sci USA 2003, I00:7977-82.

1705. Keith CT, Borisy AA and Stockwell BR: Multicomponent therapeutics for networked systems. Nat Rev Drug Discov 2005, 4:7I-8.

1706. Costello F, Stuve O, Weber MS, Zamvil SS and Frohman E: Combination therapies for multiple sclerosis: scientific rationale, clinical trials, and clinical practice. Curr Opin Neurol 2007, 20:28I-5.

1707. Lehár J, Zimmermann GR, Krueger AS, Molnar RA, Ledell JT, Heilbut AM, Short GF 3rd, Giusti LC, Nolan GP, Magid OA, Lee MS, Borisy AA, Stockwell BR and Keith CT: Chemical combination effects predict connectivity in biological systems. Mol Syst Biol 2007, 3:80.

1708. Zimmermann GR, Lehár J and Keith CT: Multi-target therapeutics: when the whole is greater than the sum of the parts. Drug Discov Today 2007, I 2:34-42.

1709. Aruoma Ol and Halliwell B: The iron-binding and hydroxyl radical scavenging action of anti-inflammatory drugs. Xenobiotica 1988, I 8:459-70.

1710. Cherny RA, Atwood CS, Xilinas ME, Gray DN, Jones WD, McLean CA, Barnham KJ, Volitakis I, Fraser FW, Kim YS, Huang XD, Goldstein LE, Moir RD, Lim JT, Beyreuther K, Zheng $\mathrm{H}$, Tanzi RE, Masters CL and Bush Al: Treatment with a copper-zinc chelator markedly and rapidly inhibits betaamyloid accumulation in Alzheimer's disease transgenic mice. Neuron 2001, 30:665-676.
17II. Di Vaira M, Bazzicalupi C, Orioli P, Messori L, Bruni B and Zatta P: Clioquinol, a drug for Alzheimer's disease specifically interfering with brain metal metabolism: structural characterization of its zinc(II) and copper(II) complexes. Inorg Chem 2004, 43:3795-7.

17I2. Shiraki H: The neuropathology of subacute myelo-opticoneuropathy, "SMON", in the humans: -with special reference to the quinoform intoxication. Jpn J Med Sci Biol I975, 28 (Suppl): I0I-64.

17I3. Arbiser JL, Kraeft SK, van Leeuwen R, Hurwitz SJ, Selig M, Dickersin GR, Flint A, Byers HR and Chen LB: Clioquinol-zinc chelate: a candidate causative agent of subacute myelooptic neuropathy. Mol Med 1998, 4:665-70.

17|4. Sai Y: Biochemical and molecular pharmacological aspects of transporters as determinants of drug disposition. Drug Metab Pharmacokinet 2005, 20:91-9.

17I5. Richardson DR and Ponka P: Orally effective iron chelators for the treatment of iron overload disease: the case for a further look at pyridoxal isonicotinoyl hydrazone and its analogs. J Lab Clin Med 1998, I32:35I-2.

17|6. Richardson DR and Ponka P: Pyridoxal isonicotinoyl hydrazone and its analogs: potential orally effective ironchelating agents for the treatment of iron overload disease. J Lab Clin Med 1998, I3 I:306-15.

1717. Buss JL, Arduini E, Shephard KC and Ponka P: Lipophilicity of analogs of pyridoxal isonicotinoyl hydrazone (PTH) determines the efflux of iron complexes and toxicity in $\mathrm{K562}$ cells. Biochem Pharmacol 2003, 65:349-360.

1718. Buss JL, Neuzil J, Gellert N, Weber C and Ponka P: Pyridoxal isonicotinoyl hydrazone analogs induce apoptosis in hematopoietic cells due to their iron-chelating properties. Biochemical Pharmacology 2003, 65:16I-I72.

1719. Lovejoy DB and Richardson DR: Iron chelators as antineoplastic agents: current developments and promise of the PIH class of chelators. Curr Med Chem 2003, I0:1035-49.

1720. Simunek T, Sterba M, Popelova O, Kaiserova H, Potacova A, Adamcova $M$, Mazurova Y, Ponka P and Gersl V: Pyridoxal isonicotinoyl hydrazone (PIH) and its analogs as protectants against anthracycline-induced cardiotoxicity. Hemoglobin 2008, 32:207-15.

|72I. Buss JL and Ponka P: Hydrolysis of pyridoxal isonicotinoyl hydrazone and its analogs. Biochim Biophys Acta 2003, |6 | 9:177-186.

1722. Šimůnek T, Boer $C$ Bouwman RA, Vlasblom $R$, Versteilen AMG Štěrba M, Geršl V, Hrdina R, Poňka P, de Lange JJ, Paulus WJ and Musters RJP: SIH - a novel lipophilic iron chelator - protects H9c2 cardiomyoblasts from oxidative stress-induced mitochondrial injury and cell death. Journal of Molecular and Cellular Cardiology 2005, 39:345-354.

1723. Kalinowski DS, Sharpe PC, Bernhardt PV and Richardson DR: Structure-Activity Relationships of Novel Iron Chelators for the Treatment of Iron Overload Disease: The Methyl Pyrazinylketone Isonicotinoyl Hydrazone Series. J Med Chem 2007.

1724. Mouralian C, Buss JL, Stranix B, Chin J and Ponka P: Mobilization of iron from cells by hydroxyquinoline-based chelators. Biochem Pharmacol 2005, 71:214-222

1725. Murakami K, Haneda $M$ and Yoshino $M$ : Prooxidant action of xanthurenic acid and quinoline compounds: role of transition metals in the generation of reactive oxygen species and enhanced formation of 8-hydroxy-2'-deoxyguanosine in DNA. Biometals 2006, 19:429-35.

1726. Murakami K, Ishida K, Watakabe K, Tsubouchi R, Naruse M and Yoshino M: Maltol/iron-mediated apoptosis in HL60 cells: participation of reactive oxygen species. Toxicol Lett 2006, | 6 | : 102-7.

1727. Youdim MBH, Stephenson G and Ben Shachar D: Ironing iron out in Parkinson's disease and other neurodegenerative diseases with iron chelators - A lesson from 6-hydroxydopamine and iron chelators, desferal and VK-28. Redox-Active Metals in Neurological Disorders, Annals of the New York Academy of Sciences 2004, I012:306-325.

1728. Ben Shachar D, Kahana N, Kampel V, Warshawsky A and Youdim $\mathrm{MBH}$ : Neuroprotection by a novel brain permeable iron chelator, VK-28, against 6-hydroxydopamine lession in rats. Neuropharmacology 2004, 46:254-263.

1729. Youdim $\mathrm{MBH}$, Fridkin $\mathrm{M}$ and Zheng $\mathrm{HL}$ : Bifunctional drug derivatives of MAO-B inhibitor rasagiline and iron chelator VK-28 as a more effective approach to treatment of brain 
ageing and ageing neurodegenerative diseases. Mech Ageing Dev 2005, 126:317-326.

1730. Bandyopadhyay S, Huang X, Cho H, Greig NH, Youdim MB and Rogers JT: Metal specificity of an iron-responsive element in Alzheimer's APP mRNA 5 ' untranslated region, tolerance of SH-SY5Y and H4 neural cells to desferrioxamine clioquinol, VK-28, and a piperazine chelator. Journal of Neural Transmission-Supplement 2006, 237-247.

1731. Hopkins AL, Mason JS and Overington JP: Can we rationally design promiscuous drugs? Curr Opin Struct Biol 2006, 16:127-36.

1732. Overington JP, Al-Lazikani B and Hopkins AL: How many drug targets are there? Nat Rev Drug Discov 2006, 5:993-6.

1733. Paolini GV, Shapland RH, van Hoorn WP, Mason JS and Hopkins AL: Global mapping of pharmacological space. Nat Biotechnol 2006, 24:805-8I5.

1734. Dey A, Tergaonkar $V$ and Lane DP: Double-edged swords as cancer therapeutics: simultaneously targeting p53 and NFkappaB pathways. Nat Rev Drug Discov 2008, 7:103|-40.

1735. Baret P, Beguin CG, Boukhalfa H, Caris C, Laulhere JP, Pierre JL and Serratrice G: O-Trensox - a promising water-soluble iron chelator (both Fe-III and Fe-II) potentially suitable for plant nutrition and iron chelation therapy. JACS 1995, I I 7:9760-976I.

1736. Caris C, Baret P, Beguin C, Serratrice G, Pierre JL and Laulhere JP: Metabolization of iron by plant cells using O-Trensox, a high-affinity abiotic iron-chelating agent. Biochem J 1995, 3 I 2 (Pt 3):879-85.

1737. Henry C, Rakba N, Imbert D, Thomas F, Baret P, Serratrice G, Gaude D, Pierre JL, Ward RJ, Crichton RR and Lescoat G: New 8hydroxyquinoline and catecholate iron chelators: Influence of their partition coefficient on their biological activity. Biochem Pharmacol 200I, 62:1355-1362.

1738. Serratrice G, Boukhalfa H, Beguin C, Baret P, Caris C and Pierre JL: O-TRENSOX, a new tripodal iron chelator based on 8hydroxyquinoline subunits: Thermodynamic and kinetic studies. Inorganic Chemistry 1997, 36:3898-39I0.

1739. Imbert D, Baret P, Gaude D, Gautier-Luneau I, Gellon G, Thomas F, Serratrice $G$ and Pierre JL: Hydrophilic and lipophilic iron chelators with the same complexing abilities. Chemistry-a European Journal 2002, 8:1091-1100.

1740. Pierre JL, Baret $P$ and Serratrice G: Hydroxyquinolines as iron chelators. Curr Med Chem 2003, 10:1077-1084.

174I. Apostol M, Baret P, Serratrice G, Desbrieres J, Putaux JL, Stebe MJ, Expert D and Pierre JL: Self-assembly of an amphiphilic iron (III) chelator: Mimicking iron acquisition in marine bacteria. Angewandte Chemie-International Edition 2005, 44:2580-2582.

1742. d'Hardemare AD, Torelli S, Serratrice G and Pierre JL: Design of iron chelators: Syntheses and iron (III) complexing abilities of tripodal tris-bidentate ligands. Biometals 2006, 19:349-366.

1743. Whitnall M, Howard J, Ponka P and Richardson DR: A class of iron chelators with a wide spectrum of potent antitumor activity that overcomes resistance to chemotherapeutics. Proc Natl Acad Sci USA 2006, 103: 1490I-6.

1744. Mello Filho AC, Hoffmann ME and Meneghini R: Cell killing and DNA damage by hydrogen peroxide are mediated by intracellular iron. Biochem J 1984, 2 I 8:273-5.

1745. Tatsuta $M$, lishi $H$, Baba M, Mikuni T, Narahara $H$, Uedo $N$ and Yano $\mathrm{H}$ : Suppression by iron chelator phenanthroline of sodium chloride-enhanced gastric carcinogenesis induced by $\mathrm{N}$-methyl-N'-nitro-N-nitrosoguanidine in Wistar rats. Cancer Lett 2003, 191:9-16.

1746. Kalinowski DS, Yu Y, Sharpe PC, Islam M, Liao YT, Lovejoy DB, Kumar N, Bernhardt PV and Richardson DR: Design synthesis, and characterization of novel iron chelators: structureactivity relationships of the 2-benzoylpyridine thiosemicarbazone series and their 3-nitrobenzoyl analogues as potent antitumor agents. I Med Chem 2007, 50:3716-29.

1747. Kalinowski DS, Sharpe PC, Bernhardt PV and Richardson DR: Design Synthesis, and Characterization of New Iron Chelators with Anti-Proliferative Activity: Structure-Activity Relationships of Novel Thiohydrazone Analogues. J Med Chem 2007, 50:6212-6225.

1748. L'Eplattenier F, Murase I and Martell AE: New multidentate ligands .6. chelating tendencies of N,N'-di(2-hydroxybenzyl) ethylenediamine-N,N'-diacetic acid. JACS 1967, 89:837.

1749. Faller B, Spanka C, Sergejew T and Tschinke V: Improving the oral bioavailability of the iron chelator HBED by breaking the symmetry of the intramolecular H-bond network. J Med Chem 2000, 43:1467-I475.
1750. Bergeron RJ, Wiegand J and Brittenham GM: HBED: The continuing development of a potential alternative to deferoxamine for iron-chelating therapy. Blood 1999, 93:370-375.

175I. Samuni AM, Afeworki M, Stein W, Yordanov AT, DeGraff W, Krishna MC, Mitchell JB and Brechbiel MW: Multifunctional antioxidant activity of HBED iron chelator. Free Radical Biology and Medicine 200I, 30:170-177.

1752. Bergeron RJ, Wiegand $J$ and Brittenham GM: HBED ligand: preclinical studies of a potential alternative to deferoxamine for treatment of chronic iron overload and acute iron poisoning. Blood 2002, 99:3019-3026.

1753. Lebedev AV, Ivanova MV and Levitsky DO: Iron chelators and free radical scavengers in naturally occurring polyhydroxylated I,4-naphthoquinones. Hemoglobin 2008, 32:165-79.

1754. Liu JK, Killilea DW and Ames BN: Age-associated mitochondrial oxidative decay: Improvement of carnitine acetyltransferase substrate-binding affinity and activity in brain by feeding old rats acetyl-L-carnitine and/or R-alpha-lipoic acid. Proc Natl Acad Sci 2002, 99:|876-|88|.

1755. Suh JH, Moreau R, Heath SH and Hagen TM: Dietary supplementation with (R)-alpha-lipoic acid reverses the agerelated accumulation of iron and depletion of antioxidants in the rat cerebral cortex. Redox Rep 2005, 10:52-60.

1756. Skibska B, Jozelowicz-Okonkwo G and Goraca A: Protective effects of early administration of alpha-lipoic acid against lipopolysaccharide-induced plasma lipid peroxidation. Pharmacological Reports 2006, 58:399-404.

1757. Holmquist L, Stuchbury G, Berbaum K, Muscat S, Young S, Hager K, Engel J and Munch G: Lipoic acid as a novel treatment for Alzheimer's disease and related dementias. Pharmacology \& Therapeutics 2007, I I3:154-164.

1758. Petersen Shay K, Moreau RF, Smith EI and Hagen TM: Is alphalipoic acid a scavenger of reactive oxygen species in vivo? Evidence for its initiation of stress signaling pathways that promote endogenous antioxidant capacity. IUBMB Life 2008, 60:362-7.

1759. Wei Y and Guo M: Hydrogen peroxide triggered prochelator activation, subsequent metal chelation, and attenuation of the fenton reaction. Angew Chem Int Ed Engl 2007, 46:4722-5.

1760. Galey JB: Potential use of iron chelators against oxidative damage. Adv Pharmacol 1997, 38:167-203.

176I. Faa G and Crisponi G: Iron chelating agents in clinical practice. Coordination Chemistry Reviews 1999, 184:29I-310.

1762. Kontoghiorghes GJ, Pattichi K, Hadjigavriel $M$ and Kolnagou $A$ : Transfusional iron overload and chelation therapy with deferoxamine and deferiprone (LI). Transfusion Science 2000, 23:2II-223.

1763. Emerit J, Beaumont C and Trivin F: Iron metabolism, free radicals, and oxidative injury. Biomed Pharmacother 200I, 55:333-9.

1764. Tam TF, Leung-Toung R, Li WR, Wang YS, Karimian K and Spino M: Iron chelator research: Past present, and future. Current Medicinal Chemistry 2003, 10:983-995.

1765. Blanuša $M$, Varnai VM, Piasek $M$ and Kostial K: Chelators as antidotes of metal toxicity: Therapeutic and experimental aspects. Curr Med Chem 2005, 12:277।-2794.

1766. Kontoghiorghes GJ and Kolnagou A: Molecular factors and mechanisms affecting iron and other metal excretion or absorption in health and disease. The role of natural and synthetic chelators. Current Medicinal Chemistry 2005, I 2:2695-2709.

1767. Kontoghiorghes G]: Iron mobilization from transferrin and non-transferrin-bound-iron by deferiprone. Implications in the treatment of thalassemia, anemia of chronic disease, cancer and other conditions. Hemoglobin 2006, 30:183-200.

1768. Golenser J, Domb A, Leshem B, Kremsner P and Luty A: Iron chelators as drugs against malaria pose a potential risk. Redox Rep 2003, 8:268-7I.

1769. Cherny RA, Legg JT, McLean CA, Fairlie DP, Huang XD, Atwood CS, Beyreuther K, Tanzi RE, Masters CL and Bush Al: Aqueous dissolution of Alzheimer's disease $A$ beta amyloid deposits by biometal depletion. J Biol Chem 1999, 274:23223-23228.

1770. Ritchie CW, Bush Al, Mackinnon A Macfarlane S, Mastwyk M, MacGregor L, Kiers L, Cherny R, Li QX, Tammer A, Carrington D, Mavros C, Volitakis I, Xilinas M, Ames D, Davis S, Volitakis I, Xilinas M. Ames D, Davis S, Beyreuther K, Tanzi RE and Masters CL: Metal-protein attenuation with iodochlorhydroxyquin (clioquinol) targeting $\mathrm{A}$ beta amyloid deposition 
and toxicity in Alzheimer disease - A pilot phase 2 clinical trial. Archives of Neurology 2003, 60:1685-169|.

I77I. Regland B, Lehmann W, Abedini I, Blennow $K$, Jonsson $M$, Karlsson I, Sjogren M, Wallin A, Xilinas M and Gottfries CG: Treatment of Alzheimer's disease with clioquinol. Dementia and Geriatric Cognitive Disorders 200 I, I 2:408-4I4.

1772. Kaur D, Yantiri F, Rajagopalan S, Kumar J, Mo JO, Boonplueang R, Viswanath V, Jacobs R, Yang L, Beal MF, DiMonte D, Volitaskis I, Ellerby L, Cherny RA, Bush Al and Andersen JK: Genetic or pharmacological iron chelation prevents MPTP-induced neurotoxicity in vivo: A novel therapy for Parkinson's disease. Neuron 2003, 37:899-909.

I773. Ikeda $Y$, Ikeda $\mathrm{K}$ and Long DM: Comparative study of different iron-chelating agents in cold-induced brain edema. Neurosurgery 1989, 24:820-4.

1774. Masuda T, Hida H, Kanda Y, Aihara N, Ohta K, Yamada K and Nishino $\mathrm{H}$ : Oral administration of metal chelator ameliorates motor dysfunction after a small hemorrhage near the internal capsule in rat. J Neurosci Res 2007, 85:213-222.

1775. De Vries B, Walter SJ, Von Bonsdorff L, Wolfs TGAM, Van Heurn LWE, Parkkinen J and Buurman WA: Reduction of circulating redox-active iron by apotransferrin protects against renal ischemia-reperfusion injury. Transplantation 2004, 77:669-675.

1776. Galey JB, Destree O, Dumats J, Pichaud P, Marche J, Genard S, Bracciolli G, Le Capitaine L, Plessix H, Brambilla L and Cantoni O: Protection of U937 cells against oxidative injury by a nove series of iron chelators. Free Radic Biol Med 1998, 25:88I-90.

1777. Galey JB, Destree O, Dumats J, Genard S and Tachon P: Protection against oxidative damage by iron chelators: effect of lipophilic analogues and prodrugs of $\mathbf{N}, \mathbf{N}^{\prime}$-bis $(3,4$ 5-trimethoxybenzyl)ethylenediamine- $\mathbf{N}, \mathbf{N}$ '-diacetic acid (ORIOI4I). J Med Chem 2000, 43:|4I8-2I.

1778. Galey JB: Recent advances in the design of iron chelators against oxidative damage. Mini Rev Med Chem 200I, I:233-42.

1779. Charkoudian LK, Pham DM and Franz KJ: A pro-chelator triggered by hydrogen peroxide inhibits iron-promoted hydroxyl radical formation. JACS 2006, I 28: | 2424-I2425.

1780. Gal S, Zheng $H$, Fridkin $M$ and Youdim MBH: Novel multifunctional neuroprotective iron chelator-monoamine oxidase inhibitor drugs for neurodegenerative diseases. In vivo selective brain monoamine oxidase inhibition and prevention of MPTP-induced striatal dopamine depletion. Journal of Neurochemistry 2005, 95:79-88.

I78I. Zheng H, Weiner LM, Bar-Am O, Epsztejn S, Cabantchik ZI, Warshawsky A, Youdim MB and Fridkin M: Design synthesis, and evaluation of novel bifunctional iron-chelators as potential agents for neuroprotection in Alzheimer's, Parkinson's, and other neurodegenerative diseases. Bioorg Med Chem 2005, I 3:773-83.

1782. Zheng HL, Youdim MBH, Weiner LM and Fridkin M: Novel potential neuroprotective agents with both iron chelating and amino acid-based derivatives targeting central nervous system neurons. Biochemical Pharmacology 2005, 70:1642-I652.

I783. Gal S, Fridkin M, Amit T, Zheng $\mathrm{H}$ and Youdim MBH: M30, a novel multifunctional neuroprotective drug with potent iron chelating and brain selective monoamine oxidase-ab inhibitory activity for Parkinson's disease. J Neural Transmission - Suppl 2006, 447-456.

1784. Avramovich-Tirosh Y, Amit T, Bar-Am O, Zheng HL, Fridkin M and Youdim $\mathrm{MBH}$ : Therapeutic targets and potential of the novel brain- permeable multifunctional iron chelator-monoamine oxidase inhibitor drug, $M-30$, for the treatment of Alzheimer's disease. I Neurochem 2007, I 00:490-502.

1785. Kayyali R, Pannala AS, Khodr H and Hider RC: Comparative radical scavenging ability of bidentate iron (III) chelators. Biochem Pharmacol 1998, 55:1327-32.

1786. Kontoghiorghes G] and Weinberg ED: Iron - mammalian defense systems, mechanisms of disease, and chelation therapy approaches. Blood Rev 1995, 9:33-45.

1787. Gabutti $V$ and Piga A: Results of long-perm iron-chelating therapy. Acta Haematologica 1996, 95:26-36.

1788. Song BB, Anderson DJ and Schacht J: Protection from gentamicin ototoxicity by iron chelators in guinea pig in vivo. Journal of Pharmacology and Experimental Therapeutics 1997, 282:369-377.

1789. Song BB and Schacht J: Variable efficacy of radical scavengers and iron chelators to attenuate gentamicin ototoxicity in guinea pig in vivo. Hearing Research 1996, 94:87-93.
1790. Wetli HA, Buckett PD and Wessling-Resnick M: Small-molecule screening identifies the selanazal drug ebselen as a potent inhibitor of DMTI-mediated iron uptake. Chemistry \& Biology 2006, I 3:965-972.

|79|. Hovorka SW and Schoneich C: Oxidative degradation of pharmaceuticals: Theory, mechanisms and inhibition. J Pharm Sci 2001, 90:253-269.

1792. England K, Driscoll CO and Cotter TG: ROS and protein oxidation in early stages of cytotoxic drug induced apoptosis. Free Radical Research 2006, 40: I I 24-II37.

1793. Cha'on U, Valmas N, Collins PJ, Reilly PEB, Hammock BD and Ebert PR: Disruption of iron homeostasis increases phosphine toxicity in Caenorhabditis elegans. Toxicological Sciences 2007, 96:194-201.

1794. Bus JS, Gibson JE and Aust SD: Superoxide-catalyzed and singlet oxygen-catalyzed lipid peroxidation as a possible mechanism for paraquat (methyl viologen) toxicity. Biochem Biophys Res Commun 1974, 58:749-755.

1795. Doelman CJA and Bast A: Oxygen Radicals in Lung Pathology. Free Rad Biol Med 1990, 9:381-400.

1796. Hippeli $S$ and Elstner EF: Transition metal ion-catalyzed oxygen activation during pathogenic processes. Febs Letters 1999, 443:I-7.

1797. Jones GM and Vale JA: Mechanisms of toxicity, clinical features, and management of diquat poisoning: A review. J Toxicol 2000, 38:123-128.

1798. Suntres ZE: Role of antioxidants in paraquat toxicity. Toxicology 2002, I 80:65-77.

1799. Abdollahi M, Ranjbar A, Shadnia S, Nikfar S and Rezaie A: Pesticides and oxidative stress: a review. Medical Science Monitor 2004, 10:RA|4I-RAI47.

1800. Rogachev I, Kampel V, Gusis V, Cohen N, Gressel J and Warshawsky A: Synthesis properties, and use of copperchelating amphiphilic dithiocarbamates as synergists of oxidant-generating herbicides. Pesticide Biochemistry and Physiology 1998, 60:133-145.

I80I. Kotamraju S, Chitambar CR, Kalivendi SV, Joseph J and Kalyanaraman B: Transferrin receptor-dependent iron uptake is responsible for doxorubicin-mediated apoptosis in endothelial cells - Role of oxidant-induced iron signaling in apoptosis. Journal of Biological Chemistry 2002, 277:17179-17187.

1802. Minotti G, Menna P, Salvatorelli E, Cairo G and Gianni L: Anthracyclines: Molecular advances and pharmacologic developments in antitumor activity and cardiotoxicity. Pharmacological Reviews 2004, 56:185-229.

1803. Asare GA, Bronz M, Naidoo $V$ and Kew MC: Interactions between aflatoxin B I and dietary iron overload in hepatic mutagenesis. Toxicology 2007, 234:157-66.

1804. Scalbert A and Williamson G: Dietary intake and bioavailability of polyphenols. I Nutr 2000, I 30:2073S-85S

1805. Reed J: Cranberry flavonoids, atherosclerosis and cardiovascular health. Crit Rev Food Sci Nutr 2002, 42:30I-16.

1806. Benzie IF: Evolution of dietary antioxidants. Comp Biochem Physiol A Mol Integr Physiol 2003, I36: I I3-26.

1807. Higdon JV and Frei B: Tea catechins and polyphenols: health effects metabolism, and antioxidant functions. Crit Rev Food Sci Nutr 2003, 43:89-|43.

I808. Surh YJ: Cancer chemoprevention with dietary phytochemicals. Nat Rev Cancer 2003, 3:768-80.

1809. Manach C, Scalbert A, Morand C, Remesy C and Jimenez L: Polyphenols: food sources and bioavailability. Am J Clin Nutr 2004, 79:727-47.

1810. Manach C, Mazur A and Scalbert A: Polyphenols and prevention of cardiovascular diseases. Curr Opin Lipidol 2005, I 6:77-84.

I8II. Scalbert A, Manach C, Morand C, Remesy C and Jimenez L: Dietary polyphenols and the prevention of diseases. Crit Rev Food Sci Nutr 2005, 45:287-306.

18I2. Nichenametla SN, Taruscio TG, Barney DL and Exon JH: A review of the effects and mechanisms of polyphenolics in cancer. Crit Rev Food Sci Nutr 2006, 46: I6I-83.

1813. Zhang HY, Yang DP and Tang GY: Multipotent antioxidants: from screening to design. Drug Discov Today 2006, I I:749-54.

1814. Shahidi F and Wanasundara PK. Phenolic antioxidants. Crit Rev Food Sci Nutr 1992, 32:67-103.

18I5. Wright JS, Johnson ER and DiLabio GA: Predicting the activity of phenolic antioxidants: theoretical method, analysis of substituent effects, and application to major families of antioxidants. J Am Chem Soc 2001, I 23: I I73-83. 
1816. Block G, Patterson B and Subar A: Fruit vegetables, and cancer prevention: a review of the epidemiological evidence. Nutr Cancer 1992, 18:1-29.

1817. Ness AR and Powles JW: Fruit and vegetables, and cardiovascular disease: a review. Int I Epidemiol 1997, 26: I-I3.

1818. Krauss RM, Eckel RH, Howard B, Appel LJ, Daniels SR, Deckelbaum RJ, Erdman JW Jr, Kris-Etherton P, Goldberg IJ, Kotchen TA, Lichtenstein AH, Mitch WE, Mullis R, Robinson K, Wylie-Rosett J, St Jeor S, Suttie J, Tribble DL and Bazzarre TL: AHA Dietary Guidelines: revision 2000: A statement for healthcare professionals from the Nutrition Committee of the American Heart Association. Circulation 2000, 102:2284-99.

1819. Yang CS, Landau JM, Huang MT and Newmark HL: Inhibition of carcinogenesis by dietary polyphenolic compounds. Annu Rev Nutr 200I, 21:381-406.

1820. Lotito SB and Frei B: Consumption of flavonoid-rich foods and increased plasma antioxidant capacity in humans: Cause consequence, or epiphenomenon? Free Rad Biol Med 2006, 41: | 727-I746.

I82 I. Cook NC and Samman S: Flavonoids - Chemistry metabolism, cardioprotective effects, and dietary sources. Journal of Nutritional Biochemistry 1996, 7:66-76.

1822. Bravo L: Polyphenols: chemistry, dietary sources metabolism, and nutritional significance. Nutrition Reviews 1998, 56:317-333

1823. Fang $Y Z$, Yang $S$ and $W u$ GY: Free radicals antioxidants, and nutrition. Nutrition 2002, I 8:872-879.

1824. Fraga CG and Oteiza PI: Iron toxicity and antioxidant nutrients. Toxicology 2002, I 80:23-32

1825. Halvorsen BL, Holte K, Myhrstad MC, Barikmo I, Hvattum E, Remberg SF, Wold AB, Haffner K, Baugerod H, Andersen LF, Moskaug O, Jacobs DR Jr and Blomhoff R: A systematic screening of total antioxidants in dietary plants. J Nutr 2002, I32:46|-7|.

1826. Havsteen $\mathrm{BH}$ : The biochemistry and medical significance of the flavonoids. Pharmacol Ther 2002, 96:67-202.

1827. Vertuani S, Angusti $A$ and Manfredini S: The antioxidants and pro-antioxidants network: an overview. Curr Pharmaceut Design 2004, I0:1677-1694.

1828. Arts ICW and Hollman PCH: Polyphenols and disease risk in epidemiologic studies. American Journal of Clinical Nutrition 2005, $81: 317 S-325 S$

1829. Afanas'ev IB, Dorozhko Al, Brodskii AV, Kostyuk VA and Potapovitch Al: Chelating and free radical scavenging mechanisms of inhibitory action of rutin and quercetin in lipid peroxidation. Biochem Pharmacol 1989, 38:1763-9.

1830. Morel I, Lescoat G, Cillard P and Cillard J: Role of flavonoids and iron chelation in antioxidant action. Methods Enzymol 1994, 234:437-43.

I83 I. Moran JF, Klucas RV, Grayer RJ, Abian J and Becana M: Complexes of iron with phenolic compounds from soybean nodules and other legume tissues: prooxidant and antioxidant properties. Free Radic Biol Med 1997, 22:86I-70.

1832. Yoshino $M$ and Murakami $K$ : Interaction of iron with polyphenolic compounds: application to antioxidant characterization. Anal Biochem 1998, 257:40-4.

1833. Grinberg LN, Newmark H, Kitrossky N, Rahamim E, Chevion M and Rachmilewitz EA: Protective effects of tea polyphenols against oxidative damage to red blood cells. Biochemical Pharmacology 1997, 54:973-978.

1834. Lopes GKB, Schulman HM and Hermes-Lima M: Polyphenol tannic acid inhibits hydroxyl radical formation from Fenton reaction by complexing ferrous ions. Biochimica Et Biophysica Acta-General Subjects 1999, |472: |42-152.

1835. Packer L, Rimbach G and Virgili F: Antioxidant activity and biologic properties of a procyanidin-rich extract from pine (Pinus maritima) bark, pycnogenol. Free Rad Biol Med I999, 27:704-724.

1836. Robards K, Prenzler PD, Tucker G, Swatsitang P and Glover W: Phenolic compounds and their role in oxidative processes in fruits. Food Chem 1999, 66:401-436.

1837. Middleton E, Kandaswami C and Theoharides TC: The effects of plant flavonoids on mammalian cells: Implications for inflammation, heart disease, and cancer. Pharmacological Reviews 2000, 52:673-75I

1838. Hider RC, Liu ZD and Khodr $\mathrm{HH}$ : Metal chelation of polyphenols. Flavonoids and Other Polyphenols, Methods in Enzymology 200I, 335:190-203.
1839. Levites Y, Youdim MBH, Maor G and Mandel S: Attenuation of 6hydroxydopamine (6-OHDA)-induced nuclear factor-kappaB (NF-kappa B) activation and cell death by tea extracts in neuronal cultures. Biochemical Pharmacology 2002, 63:21-29.

1840. Mandel S and Youdim MBH: Catechin polyphenols: Neurodegeneration and neuroprotection in neurodegenerative diseases. Free Radical Biology and Medicine 2004, 37:304-3I 7.

184I. Mandel S, Weinreb $\mathrm{O}$, Amit $\mathrm{T}$ and Youdim $\mathrm{MBH}$ : Cell signaling pathways in the neuroprotective actions of the green tea polyphenol (-)-epigallocatechin-3-gallate: implications for neurodegenerative diseases. J Neurochem 2004, 88: I555-I569.

1842. Hague T, Andrews PLR, Barker J and Naughton DP: Dietary chelators as antioxidant enzyme mimetics: implications for dietary intervention in neurodegenerative diseases. Behavioural Pharmacology 2006, I 7:425-430.

1843. Weinreb O, Amit $T$ and Youdim MB: A novel approach of proteomics and transcriptomics to study the mechanism of action of the antioxidant-iron chelator green tea polyphenol (-)-epigallocatechin-3-gallate. Free Radic Biol Med 2007, 43:546-56.

1844. Rice-Evans CA, Miller NJ and Paganga G: Structure-antioxidant activity relationships of flavonoids and phenolic acids. Free Radic Biol Med 1996, 20:933-56.

1845. Rice-Evans CA, Miller J and Paganga G: Antioxidant properties of phenolic compounds. Trends in Plant Science 1997, 2:152-159.

1846. Kähkönen MP, Hopia AI, Vuorela HJ, Rauha JP, Pihlaja K, Kujala TS and Heinonen $\mathrm{M}$ : Antioxidant activity of plant extracts containing phenolic compounds. J Agric Food Chem 1999, 47:3954-62.

1847. Wang H, Cao GH and Prior RL: Oxygen radical absorbing capacity of anthocyanins. Journal of Agricultural and Food Chemistry 1997, 45:304-309.

1848. Harborne JB and Williams CA: Advances in flavonoid research since 1992. Phytochemistry 2000, 55:48I-504.

1849. Nijveldt RJ, van Nood E, van Hoorn DEC, Boelens PG, van Norren $\mathrm{K}$ and van Leeuwen PAM: Flavonoids: a review of probable mechanisms of action and potential applications. American Journal of Clinical Nutrition 200 I, 74:4 I 8-425.

1850. Heim KE, Tagliaferro AR and Bobilya DJ: Flavonoid antioxidants: chemistry, metabolism and structure-activity relationships. I Nutr Biochem 2002, 13:572-584.

185I. Rice-Evans CA and Packer L: Flavonoids in health and disease Marcel Dekker, New York; 22003.

1852. Robbins RJ: Phenolic acids in foods: an overview of analytical methodology. J Agric Food Chem 2003, 51:2866-87.

I853. Sakakibara H, Honda $\mathrm{Y}$, Nakagawa S, Ashida $\mathrm{H}$ and Kanazawa K: Simultaneous determination of all polyphenols in vegetables fruits, and teas. I Agric Food Chem 2003, 5I:57I-8I.

1854. Morel I, Lescoat G, Cogrel P, Sergent O, Pasdeloup N, Brissot P, Cillard $P$ and Cillard J: Antioxidant and iron-chelating activities of the flavonoids catechin, quercetin and diosmetin on iron-loaded rat hepatocyte cultures. Biochem Pharmacol 1993, 45:13-19.

1855. Ferrali M, Signorini C, Caciotti B, Sugherini L, Ciccoli L, Giachetti D and Comporti $M$ : Protection against oxidative damage of erythrocyte membrane by the flavonoid quercetin and its relation to iron chelating activity. FEBS Lett |997, 4 | 6: I23-9.

1856. Shoskes DA: Effect of bioflavonoids quercetin and curcumin on ischemic renal injury - A new class of renoprotective agents. Transplantation 1998, 66:147-152.

1857. Kostyuk VA and Potapovich Al: Antiradical and chelating effects in flavonoid protection against silica-induced cell injury. Archives of Biochemistry and Biophysics 1998, 355:43-48.

1858. Aherne SA and O'Brien NM: Mechanism of protection by the flavonoids, quercetin and rutin, against tert-butylhydroperoxide- and menadione induced DNA single strand breaks in Caco-2 cells. Free Radical Biology and Medicine 2000, 29:507-5 |4.

1859. Cheng IF and Breen K: On the ability of four flavonoids baicilein, luteolin naringenin, and quercetin, to suppress the Fenton reaction of the iron-ATP complex. Biometals 2000, 13:77-83.

1860. Zhang Y, Li H, Zhao Y and Gao Z: Dietary supplementation of baicalin and quercetin attenuates iron overload induced mouse liver injury. Eur J Pharmacol 2006, 535:263-9.

186I. Comporti M, Signorini C, Buonocore G and Ciccoli L: Iron release, oxidative stress and erythrocyte ageing. Free Radical Biology and Medicine 2002, 32:568-576.

1862. Mira L, Fernandez MT, Santos M, Rocha R, Florencio MH and Jennings KR: Interactions of flavonoids with iron and copper 
ions: A mechanism for their antioxidant activity. Free Radical Research 2002, 36: I|99-1208.

1863. Melidou M, Riganakos K and Galaris D: Protection against nuclear DNA damage offered by flavonoids in cells exposed to hydrogen peroxide: The role of iron chelation. Free Rad Biol Med 2005, 39:159|-1600.

1864. El Hajji H, Nkhili E, Tomao $V$ and Dangles $O$ : Interactions of quercetin with iron and copper ions: complexation and autoxidation. Free Radic Res 2006, 40:303-20.

1865. Russo A, Acquaviva R, Campisi A, Sorrenti V, Di Giacomo C, Virgata $G$, Barcellona $M L$ and Vanella $A$ : Bioflavonoids as antiradicals, antioxidants and DNA cleavage protectors. Cell Biology and Toxicology 2000, 16:91-98.

1866. Kaiserová $H$, Šimůnek $T$, Vijgh van der WJ, Bast $A$ and Kvasničková E: Flavonoids as protectors against doxorubicin cardiotoxicity: role of iron chelation, antioxidant activity and inhibition of carbonyl reductase. Biochim Biophys Acta 2007, I 772: 1065-74.

1867. Zhao Y, Li H, Gao Z and Xu H: Effects of dietary baicalin supplementation on iron overload-induced mouse liver oxidative injury. Eur J Pharmacol 2005, 509: I95-200.

1868. Borsari M, Ferrari E, Grandi R and Saladini M: Curcuminoids as potential new iron-chelating agents: spectroscopic, polarographic and potentiometric study on their Fe(III) complexing ability. Inorganica Chimica Acta 2002, 328:6I-68.

1869. Baum L and $\mathrm{Ng} \mathrm{A}$ : Curcumin interaction with copper and iron suggests one possible mechanism of action in Alzheimer's disease animal models. J Alzheimers Dis 2004, 6:367-377

1870. Bernabé-Pineda M, Ramírez-Silva MT, Romero-Romo MA, González-Vergara E and Rojas-Hernández A: Spectrophotometric and electrochemical determination of the formation constants of the complexes Curcumin-Fe(III)-water and Curcumin-Fe (II)-water. Spectrochim Acta A 2004, 60: I I 05-I I I3.

187I. Jiao Y, Wilkinson J, Pletsch EC, Buss JL, Wang W, Planalp R, Torti FM and Torti SV: Iron chelation in the biological activity of curcumin. Free Radical Biology and Medicine 2006 , 40: I I52-I 160.

1872. Aggarwal BB, Sundaram C, Malani N and Ichikawa $H$ : Curcumin: The Indian solid gold. Adv Exp Med Biol 2007, 595:I-75.

1873. Farombi EO and Nwaokeafor IA: Anti-oxidant mechanisms of kolaviron: studies on serum lipoprotein oxidation, metal chelation and oxidative membrane damage in rats. Clin Exp Pharmacol Physiol 2005, 32:667-74.

1874. Engelmann MD, Hutcheson R and Cheng IF: Stability of ferric complexes with 3-hydroxyflavone (flavonol), 5,7-dihydroxyflavone (chrysin), and 3', 4'-dihydroxyflavone. J Agric Food Chem 2005, 53:2953-2960.

1875. Botelho FV, Alvarez-Leite JI, Lemos VS, Pimenta AM, Calado HD, Matencio T, Miranda CT and Pereira-Maia EC: Physicochemical study of floranol, its copper(II) and iron(III) complexes, and their inhibitory effect on LDL oxidation. J Inorg Biochem 2007, | 0 |:935-43.

1876. Gottlieb M, Leal-Campanario R, Campos-Esparza MR, SanchezGomez MV, Alberdi E, Arranz A, Delgado-Garcia JM, Gruart A and Matute C: Neuroprotection by two polyphenols following excitotoxicity and experimental ischemia. Neurobiol Dis 2006, 23:374-86.

1877. Pardo-Andreu GL, Sanchez-Baldoquin C, Avila-Gonzalez R, Delgado R, Naal Z and Curti C: Fe(III) improves antioxidant and cytoprotecting activities of mangiferin. European Journal of Pharmacology 2006, 547:31-36.

1878. Pardo-Andreu GL, Cavalheiro RA, Dorta DJ, Naal Z, Delgado R, Vercesi $A E$ and Curti C: Fe(III) shifts the mitochondria permeability transition-eliciting capacity of mangiferin to protection of organelle. J Pharmacol Exp Ther 2007, 320:646-53.

1879. Pardo-Andreu GL, Barrios MF, Curti C. Hernandez I, Merino N, Lemus Y, Martinez I, Riano A and Delgado R: Protective effects of Mangifera indica $L$ extract (Vimang), and its major component mangiferin, on iron-induced oxidative damage to rat serum and liver. Pharmacol Res 2008, 57:79-86.

1880. Mandel S, Weinreb O, Reznichenko L, Kalfon L and Amit T: Green tea catechins as brain-permeable, non toxic iron chelators to "iron out iron" from the brain. Journal of Neural TransmissionSupplement 2006, 249-257.

188I. Mandel S, Amit T, Reznichenko L, Weinreb $\mathrm{O}$ and Youdim MBH: Green tea catechins as brain-permeable, natural iron chelators-antioxidants for the treatment of neurodegenerative disorders. Molecular Nutrition \& Food Research 2006. 50:229-234.
1882. Miller NJ, Castelluccio C, Tijburg L and Rice-Evans C: The antioxidant properties of theaflavins and their gallate esters-radical scavengers or metal chelators? FEBS Lett 1996, 392:40-4.

1883. Khokhar S and Apenten RKO: Iron binding characteristics of phenolic compounds: some tentative structure-activity relations. Food Chemistry 2003, 81:133-140.

1884. Limson J, Nyokong T and Daya S: The interaction of melatonin and its precursors with aluminium cadmium, copper iron, lead, and zinc: an adsorptive voltammetric study. J Pineal Res |998, 24:|5-2|

1885. Lin AM and Ho LT. Melatonin suppresses iron-induced neurodegeneration in rat brain. Free Radic Biol Med 2000, 28:904-II.

1886. Gulcin I, Buyukokuroglu ME and Kufrevioglu OI: Metal chelating and hydrogen peroxide scavenging effects of melatonin. $J$ Pineal Res 2003, 34:278-28I.

1887. Chwelatiuk E, Wlostowski T, Krasowska A and Bonda E: The effect of orally administered melatonin on tissue accumulation and toxicity of cadmium in mice. Journal of Trace Elements in Medicine and Biology 2006, I9:259-265.

1888. Belguendouz $L$, Fremont $L$ and Linard $A$ : Resveratrol inhibits metal ion-dependent and independent peroxidation of porcine low-density lipoproteins. Biochemical Pharmacology 1997, 53: I347-1355.

1889. Cadenas S and Barja G: Resveratrol melatonin, vitamin E, and PBN protect against renal oxidative DNA damage induced by the kidney carcinogen KBrO3. Free Radic Biol Med 1999, 26: $1531-7$.

1890. Hung LM, Chen JK, Huang SS, Lee RS and Su MJ: Cardioprotective effect of resveratrol, a natural antioxidant derived from grapes. Cardiovasc Res 2000, 47:549-55.

1891. Giovannini L, Migliori M, Longoni BM, Das DK, Bertelli AAE Panichi SV, Filippi $C$ and Bertelli A: Resveratrol, a polyphenol found in wine, reduces ischemia reperfusion injury in rat kidneys. J Cardiovasc Pharmacol 200I, 37:262-270.

1892. Huang SS, Tsai MC, Chih CL, Hung LM and Tsai SK: Resveratrol reduction of infarct size in Long-Evans rats subjected to focal cerebral ischemia. Life Sci 200I, 69:1057-65

1893. Howitz KT, Bitterman KJ Cohen HY, Lamming DW Lavu S Wood JG, Zipkin RE, Chung P, Kisielewski A, Zhang LL, Scherer B and Sinclair DA: Small molecule activators of sirtuins extend Saccharomyces cerevisiae lifespan. Nature 2003, 425:19|-6.

1894. Leonard SS, Xia C, Jiang BH, Stinefelt B, Klandorf H, Harris GK and Shi $X$ : Resveratrol scavenges reactive oxygen species and effects radical-induced cellular responses. Biochem Biophys Res Commun 2003, 309:1017-26.

1895. Kundu JK and Surh YJ: Molecular basis of chemoprevention by resveratrol: NF-kappaB and $\mathrm{AP}-\mathrm{I}$ as potential targets. Mutat Res 2004, 555:65-80.

1896. Signorelli $P$ and Ghidoni R: Resveratrol as an anticancer nutrient: molecular basis, open questions and promises. J Nutr Biochem 2005, 16:449-66.

1897. Baur JA, Pearson KJ Price NL Jamieson HA Lerin C Kalra A Prabhu VV, Allard JS, Lopez-Lluch G, Lewis K, Pistell PJ, Poosala S, Becker KG, Boss O, Gwinn D, Wang M, Ramaswamy S, Fishbein KW, Spencer RG, Lakatta EG, Le Couteur D, Shaw RJ, Navas $P$, Puigserver $P$, Ingram DK, de Cabo $R$ and Sinclair DA: Resveratrol improves health and survival of mice on a highcalorie diet. Nature 2006, 444:337-42.

1898. Baur JA and Sinclair DA: Therapeutic potential of resveratrol: the in vivo evidence. Nat Rev Drug Discov 2006, 5:493-506.

1899. Kotha A, Sekharam M, Cilenti L, Siddiquee K, Khaled A, Zervos AS, Carter B, Turkson J and Jove R: Resveratrol inhibits Src and Stat3 signaling and induces the apoptosis of malignant cells containing activated Stat3 protein. Mol Cancer Ther 2006, 5:621-9.

1900. Russo GL: Ins and outs of dietary phytochemicals in cancer chemoprevention. Biochem Pharmacol 2007, 74:533-44.

190I. Tsai SK, Hung LM, Fu YT, Cheng H, Nien MW, Liu HY, Zhang FB and Huang SS: Resveratrol neuroprotective effects during focal cerebral ischemia injury via nitric oxide mechanism in rats. J Vasc Surg 2007, 46:346-53.

1902. Gatz SA and Wiesmuller L: Take a break-resveratrol in action on DNA. Carcinogenesis 2008, 29:321-32

1903. Chu YF, Sun J, Wu X and Liu RH: Antioxidant and antiproliferative activities of common vegetables. J Agric Food Chem 2002, 50:6910-6.

1904. Afaq F, Saleem M, Krueger CG, Reed JD and Mukhtar $H$ : Anthocyanin- and hydrolyzable tannin-rich pomegranate 
fruit extract modulates MAPK and NF-kappaB pathways and inhibits skin tumorigenesis in CD-I mice. Int J Cancer 2005, I I 3:423-33.

1905. Malik A, Afaq F, Sarfaraz S, Adhami VM, Syed DN and Mukhtar H: Pomegranate fruit juice for chemoprevention and chemotherapy of prostate cancer. Proc Natl Acad Sci USA 2005, 102: | 4813-8.

1906. Butelli E, Titta L, Giorgio M, Mock HP, Matros A, Peterek S, Schijlen EG, Hall RD, Bovy AG, Luo J and Martin C: Enrichment of tomato fruit with health-promoting anthocyanins by expression of select transcription factors. Nat Biotechnol 2008, 26: $|30|-\mid 308$

1907. Joseph JA, Shukitt-Hale B, Denisova NA, Bielinski D, Martin A, McEwen JJ and Bickford PC: Reversals of age-related declines in neuronal signal transduction cognitive, and motor behavioral deficits with blueberry spinach, or strawberry dietary supplementation. Journal of Neuroscience 1999, |9:8||4-812|.

1908. Sweeney MI, Kalt W, MacKinnon SL, Ashby J and Gottschall Pass KT: Feeding rats diets enriched in lowbush blueberries for six weeks decreases ischemia-induced brain damage. Nutritional Neuroscience 2002, 5:427-43I.

1909. Joseph JA, Fisher DR and Bielinski D: Blueberry extract alters oxidative stress-mediated signaling in COS-7 cells transfected with selectively vulnerable muscarinic receptor subtypes. I Alzheimers Dis 2006, 9:35-42.

1910. Ramassamy C: Emerging role of polyphenolic compounds in the treatment of neurodegenerative diseases: A review of their intracellular targets. Eur J Pharmacol 2006, 545:5I-64.

1911. Kalt W, Joseph JA and Shukitt-Hale B: Blueberries and human health: a review of current reseach. J Amer Pomol Soc 2007, 61:151-160.

1912. Lau FC, Bielinski DF and Joseph JA: Inhibitory effects of blueberry extract on the production of inflammatory mediators in lipopolysaccharide-activated BV2 microglia. J Neurosci Res 2007, 85:1010-7.

1913. Neto CC: Cranberry and blueberry: evidence for protective effects against cancer and vascular diseases. Mol Nutr Food Res 2007, 5 I:652-64.

1914. McAnulty SR, McAnulty LS, Nieman DC, Dumke CL, Morrow JD, Utter AC, Henson DA, Proulx WR and George GL: Consumption of blueberry polyphenols reduces exercise-induced oxidative stress compared to vitamin C. Nutrition Research 2004, 24:209-221.

1915. Longpré F, Garneau P, Christen Y and Ramassamy C: Protection by EGb $76 \mathrm{I}$ against beta-amyloid-induced neurotoxicity: Involvement of NF-kappa B, SIRTI, and MAPKs pathways and inhibition of amyloid fibril formation. Free Rad Biol Med 2006, 4I:I78I-I794.

1916. Ramassamy C, Longpré F and Christen Y: Ginkgo biloba extract (EGb 76I) in Alzheimer's disease: Is there any evidence? Current Alzheimer Research 2007, 4:253-262.

1917. Wang Q, Simonyi A, Li WL, Sisk BA, Miller RL, MacDonald RS, Lubahn DE, Sun GY and Sun AY: Dietary grape supplement ameliorates cerebral ischemia-induced neuronal death in gerbils. Molecular Nutrition \& Food Research 2005, 49:443-45I.

1918. Curin $Y$ and Andriantsitohaina R: Polyphenols as potentia therapeutical agents against cardiovascular diseases. Pharmacological Reports 2005, 57:97-107.

1919. Kuriyama S, Shimazu T, Ohmori K, Kikuchi N, Nakaya N, Nishino $Y$, Tsubono $Y$ and Tsuji I: Green tea consumption and mortality due to cardiovascular disease cancer, and all causes in Japan - The Ohsaki Study. Jama-Journal of the American Medical Association 2006, 296: I 255-1265.

1920. Fraser ML, Mok GS and Lee AH: Green tea and stroke prevention: emerging evidence. Complement Ther Med 2007, I 5:46-53.

192I. Ikeda M, Suzuki C, Umegaki K, Saito K, Tabuchi M and Tomita T: Preventive effects of green tea catechins on spontaneous stroke in rats. Medical Science Monitor 2007, I3:BR40-BR45

1922. Nelson SK, Bose SK, Grunwald GK, Myhill P and McCord JM: The induction of human superoxide dismutase and catalase in vivo: A fundamentally new approach to antioxidant therapy. Free Radical Biology and Medicine 2006, 40:34l-347.

1923. Amidon GL, Lennernas H, Shah VP and Crison JR: A theoretical basis for a biopharmaceutic drug classification: the correlation of in vitro drug product dissolution and in vivo bioavailability. Pharm Res 1995, I2:413-20.

1924. Lennernäs $\mathrm{H}$ and Abrahamsson $\mathrm{B}$ : The use of biopharmaceutic classification of drugs in drug discovery and development: current status and future extension. J Pharm Pharmacol 2005 , 57:273-85.

1925. Wu CY and Benet LZ: Predicting drug disposition via application of BCS: transport/absorption/elimination interplay and development of a biopharmaceutics drug disposition classification system. Pharm Res 2005, 22: I I-23.

1926. Manach C, Williamson G, Morand C, Scalbert A and Remesy C: Bioavailability and bioefficacy of polyphenols in humans. I. Review of 97 bioavailability studies. Am J Clin Nutr 2005, 8I:230S-242S

1927. Williamson G and Manach C: Bioavailability and bioefficacy of polyphenols in humans. II. Review of 93 intervention studies. Am J Clin Nutr 2005, 81:243S-255S.

1928. Rechner AR, Kuhnle G, Bremner P, Hubbard GP, Moore KP and Rice-Evans CA: The metabolic fate of dietary polyphenols in humans. Free Radic Biol Med 2002, 33:220-35.

1929. Ross JA and Kasum CM: Dietary flavonoids: bioavailability, metabolic effects, and safety. Annu Rev Nutr 2002, 22: 19-34.

1930. Scalbert A, Morand C, Manach $C$ and Remesy $C$ : Absorption and metabolism of polyphenols in the gut and impact on health. Biomedicine \& Pharmacotherapy 2002, 56:276-282.

1931. Passamonti S, Vrhovsek U, Vanzo A and Mattivi F: Fast access of some grape pigments to the brain. I Agric Food Chem 2005, 53:7029-34

1932. Serra H, Mendes T, Bronze MR and Simplicio AL: Prediction of intestinal absorption and metabolism of pharmacologically active flavones and flavanones. Bioorg Med Chem 2008, 16:4009-18.

1933. Heiss E, Herhaus C, Klimo K, Bartsch H and Gerhauser C: Nuclear factor kappa B is a molecular target for sulforaphanemediated anti-inflammatory mechanisms. J Biol Chem 200I, 276:32008-15.

1934. Gerhäuser C, Klimo K, Heiss E, Neumann I, Gamal-Eldeen A, Knauft J, Liu GY, Sitthimonchai S and Frank N: Mechanism-based in vitro screening of potential cancer chemopreventive agents. Mutat Res 2003, 523-524:163-72.

1935. Manson MM: Cancer prevention - the potential for diet to modulate molecular signalling. Trends Mol Med 2003, 9: | |-8.

1936. Murakami A, Takahashi D, Hagihara K, Koshimizu K and Ohigashi H: Combinatorial effects of nonsteroidal antiinflammatory drugs and food constituents on production of prostaglandin E2 and tumor necrosis factor-alpha in RAW264.7 murine macrophages. Biosci Biotechnol Biochem 2003, 67: 1056-62.

1937. Murakami A, Takahashi D, Koshimizu K and Ohigashi H: Synergistic suppression of superoxide and nitric oxide generation from inflammatory cells by combined food factors. Mutat Res 2003, 523-524: |5|-6|.

1938. Murakami A, Matsumoto K, Koshimizu K and Ohigashi H: Effects of selected food factors with chemopreventive properties on combined lipopolysaccharide- and interferon-gammainduced IkappaB degradation in RAW264.7 macrophages. Cancer Lett 2003, 195:17-25.

1939. Surh YJ and Ferguson LR: Dietary and medicinal antimutagens and anticarcinogens: molecular mechanisms and chemopreventive potential-highlights of a symposium. Mutat Res 2003, 523-524: I-8.

1940. Alcaraz MJ, Vicente AM, Araico A, Dominguez JN, Terencio MC and Ferrandiz ML: Role of nuclear factor-kappaB and heme oxygenase-I in the mechanism of action of an antiinflammatory chalcone derivative in RAW 264.7 cells. $\mathrm{Br} J$ Pharmacol 2004, 142: I|91-9.

194I. Dorai T and Aggarwal BB: Role of chemopreventive agents in cancer therapy. Cancer Lett 2004, 2 I 5:129-40.

1942. Feng R, Bowman LL, Lu Y, Leonard SS, Shi X, Jiang BH, Castranova $\mathrm{V}$, Vallyathan $\mathrm{V}$ and Ding $\mathrm{M}$ : Blackberry extracts inhibit activating protein I activation and cell transformation by perturbing the mitogenic signaling pathway. Nutr Cancer 2004, 50:80-9.

1943. Goyarzu P, Malin DH, Lau FC, Taglialatela G, Moon WD Jennings R, Moy E, Moy D, Lippold S, Shukitt-Hale B and Joseph JA: Blueberry supplemented diet: effects on object recognition memory and nuclear factor-kappa $B$ levels in aged rats. Nutr Neurosci 2004, 7:75-83.

1944. Jeong WS, Kim IW, Hu R and Kong AN: Modulatory properties of various natural chemopreventive agents on the activation of NF-kappaB signaling pathway. Pharm Res 2004, 2 I:66I-70. 
1945. Park OJ and Surh YJ: Chemopreventive potential of epigallocatechin gallate and genistein: evidence from epidemiological and laboratory studies. Toxicol Lett 2004, I 50:43-56.

1946. Pham NA, Jacobberger JW, Schimmer AD, Cao P, Gronda M and Hedley DW: The dietary isothiocyanate sulforaphane targets pathways of apoptosis, cell cycle arrest, and oxidative stress in human pancreatic cancer cells and inhibits tumor growth in severe combined immunodeficient mice. Mol Cancer Ther 2004, 3: | 239-48.

1947. Zhang Y: Cancer-preventive isothiocyanates: measurement of human exposure and mechanism of action. Mutat Res 2004, 555: 173-90.

1948. Bai SK, Lee SJ, Na HJ, Ha KS, Han JA, Lee H, Kwon YG, Chung CK and Kim YM: Beta-carotene inhibits inflammatory gene expression in lipopolysaccharide-stimulated macrophages by suppressing redox-based NF-kappaB activation. Exp Mol Med 2005, 37:323-34

1949. Chen C and Kong AN: Dietary cancer-chemopreventive compounds: from signaling and gene expression to pharmacological effects. Trends Pharmacol Sci 2005, 26:318-26.

1950. Kundu JK and Surh YJ: Breaking the relay in deregulated cellular signal transduction as a rationale for chemoprevention with anti-inflammatory phytochemicals. Mutat Res 2005, 59 I: | 23-46.

195I. Losso JN and Bawadi HA: Hypoxia inducible factor pathways as targets for functional foods. I Agric Food Chem 2005, 53:375i-68.

1952. Ramos S, Alia M, Bravo L and Goya L: Comparative effects of food-derived polyphenols on the viability and apoptosis of a human hepatoma cell line (HepG2). J Agric Food Chem 2005, 53: $|27|-80$.

1953. Surh YJ, Kundu JK, Na HK and Lee JS: Redox-sensitive transcription factors as prime targets for chemoprevention with anti-inflammatory and antioxidative phytochemicals. J Nutr 2005, I35:2993S-300IS.

1954. Xu C, Shen G, Chen C, Gelinas C and Kong AN: Suppression of NF-kappaB and NF-kappaB-regulated gene expression by sulforaphane and PEITC through IkappaBalpha, IKK pathway in human prostate cancer PC-3 cells. Oncogene 2005, 24:4486-95.

1955. Zhang Y, Li J and Tang L: Cancer-preventive isothiocyanates: dichotomous modulators of oxidative stress. Free Radic Biol Med 2005, 38:70-7.

1956. Wang SY, Feng R, Lu Y, Bowman L and Ding M: Inhibitory effect on activator protein-I, nuclear factor-kappaB, and cell transformation by extracts of strawberries (Fragaria $x$ ananassa Duch.). J Agric Food Chem 2005, 53:4I87-93.

1957. Aggarwal BB and Shishodia S: Molecular targets of dietary agents for prevention and therapy of cancer. Biochem Pharmacol 2006, 71:|397-42|.

1958. Brigelius-Flohé $R$ and Banning A: Sulforaphane and selenium, partners in adaptive response and prevention of cancer. Free Radic Res 2006, 40:775-87.

1959. Jakubíkova J, Sedlák J, Bod'o J and Bao Y: Effect of isothiocyanates on nuclear accumulation of NF-kappaB, Nrf2, and thioredoxin in caco-2 cells. J Agric Food Chem 2006, 54: 1656-62.

1960. Jacob C: A scent of therapy: pharmacological implications of natural products containing redox-active sulfur atoms. Nat Prod Rep 2006, 23:85I-63.

196I. Keum YS, Yu S, Chang PP, Yuan X, Kim JH, Xu C, Han J, Agarwal A and Kong AN: Mechanism of action of sulforaphane: inhibition of p38 mitogen-activated protein kinase isoforms contributing to the induction of antioxidant response element-mediated heme oxygenase-I in human hepatoma HepG2 cells. Cancer Res 2006, 66:8804-13.

1962. Köhle C and Bock KW: Activation of coupled Ah receptor and Nrf2 gene batteries by dietary phytochemicals in relation to chemoprevention. Biochem Pharmacol 2006, 72:795-805.

1963. Kresty LA, Frankel WL, Hammond CD, Baird ME, Mele JM, Stoner GD and Fromkes |l: Transitioning from preclinical to clinical chemopreventive assessments of lyophilized black raspberries: interim results show berries modulate markers of oxidative stress in Barrett's esophagus patients. Nutr Cancer 2006, 54:|48-56.

1964. Lee KW, Kundu JK, Kim SO, Chun KS, Lee HJ and Surh YJ: Cocoa polyphenols inhibit phorbol ester-induced superoxide anion formation in cultured HL-60 cells and expression of cyclooxygenase-2 and activation of NF-kappaB and MAPKs in mouse skin in vivo. J Nutr 2006, I36: I I 50-5.
1965. Lu H, Li J, Zhang D, Stoner GD and Huang C: Molecular mechanisms involved in chemoprevention of black raspberry extracts: from transcription factors to their target genes. Nutr Cancer 2006, 54:69-78.

1966. Martin KR: Targeting apoptosis with dietary bioactive agents. Exp Biol Med 2006, 23 I: I I 7-29.

1967. Na HK and Surh YJ: Transcriptional regulation via cysteine thiol modification: a novel molecular strategy for chemoprevention and cytoprotection. Mol Carcinog 2006, 45:368-80.

1968. Pappa G, Lichtenberg M, lori R, Barillari J, Bartsch H and Gerhauser C: Comparison of growth inhibition profiles and mechanisms of apoptosis induction in human colon cancer cell lines by isothiocyanates and indoles from Brassicaceae. Mutat Res 2006, 599:76-87.

1969. Sandur SK, Ichikawa H, Sethi G, Ahn KS and Aggarwal BB: Plumbagin (5-hydroxy-2-methyl-I,4-naphthoquinone) suppresses NF-kappaB activation and NF-kappaB-regulated gene products through modulation of p65 and IkappaBalpha kinase activation, leading to potentiation of apoptosis induced by cytokine and chemotherapeutic agents. J Biol Chem 2006, 28I: I 7023-33.

1970. Shen G, Xu C, Hu R, Jain MR, Gopalkrishnan A, Nair S, Huang MT, Chan JY and Kong AN: Modulation of nuclear factor E2related factor 2-mediated gene expression in mice liver and small intestine by cancer chemopreventive agent curcumin. Mol Cancer Ther 2006, 5:39-5I.

197I. Thangapazham RL, Sharma A and Maheshwari RK: Multiple molecular targets in cancer chemoprevention by curcumin. Aaps J 2006, 8:E443-9.

1972. Vina J, Borras C, Gomez-Cabrera MC and Orr WC: Role of reactive oxygen species and (phyto)oestrogens in the modulation of adaptive response to stress. Free Radic Res 2006, 40: II|-9.

1973. Davis CD and Milner JA: Biomarkers for diet and cancer prevention research: potentials and challenges. Acta Pharmacol Sin 2007, 28: I 262-73

1974. Higdon JV, Delage B, Williams DE and Dashwood RH: Cruciferous vegetables and human cancer risk: epidemiologic evidence and mechanistic basis. Pharmacol Res 2007, 55:224-36.

1975. Huber WW and Parzefall W: Thiols and the chemoprevention of cancer. Curr Opin Pharmacol 2007, 7:404-9.

1976. Juge N, Mithen RF and Traka M: Molecular basis for chemoprevention by sulforaphane: a comprehensive review. Cel Mol Life Sci 2007, 64: I 105-27.

1977. Kampa M, Nifli AP, Notas G and Castanas E: Polyphenols and cancer cell growth. Rev Physiol Biochem Pharmacol 2007, I 59:79-I|3.

1978. Kwon KH, Barve A, Yu S, Huang MT and Kong AN: Cancer chemoprevention by phytochemicals: potential molecular targets, biomarkers and animal models. Acta Pharmacol Sin 2007, 28: |409-2|.

1979. Liby KT, Yore MM and Sporn MB: Triterpenoids and rexinoids as multifunctional agents for the prevention and treatment of cancer. Nat Rev Cancer 2007, 7:357-69.

1980. Murakami A and Ohigashi $\mathrm{H}$ : Targeting NOX, INOS and COX2 in inflammatory cells: chemoprevention using food phytochemicals. Int J Cancer 2007, I 2 I:2357-63.

198I. Nair S, Li W and Kong AN: Natural dietary anti-cancer chemopreventive compounds: redox-mediated differential signaling mechanisms in cytoprotection of normal cells versus cytotoxicity in tumor cells. Acta Pharmacol Sin 2007, 28:459-72.

1982. Neto CC: Cranberry and its phytochemicals: a review of in vitro anticancer studies. I Nutr 2007, I37:186S-193S.

1983. Syed DN, Afaq $F$ and Mukhtar H: Pomegranate derived products for cancer chemoprevention. Semin Cancer Biol 2007, I 7:377-85.

1984. Zafra-Stone S, Yasmin T, Bagchi M, Chatterjee A, Vinson JA and Bagchi D: Berry anthocyanins as novel antioxidants in human health and disease prevention. Mol Nutr Food Res 2007, 5 I:675-83.

1985. Gopalakrishnan A and Tony Kong AN: Anticarcinogenesis by dietary phytochemicals: cytoprotection by Nrf2 in normal cells and cytotoxicity by modulation of transcription factors NF-kappa B and AP-I in abnormal cancer cells. Food Chem Toxicol 2008, 46: I257-70.

1986. Nair S, Hebbar V, Shen G, Gopalakrishnan A, Khor TO, Yu S, Xu C and Kong AN: Synergistic effects of a combination of dietary factors sulforaphane and (-) epigallocatechin-3-gallate in 
HT-29 AP-I human colon carcinoma cells. Pharm Res 2008, 25:387-99.

1987. Neto CC, Amoroso JW and Liberty AM: Anticancer activities of cranberry phytochemicals: An update. Mol Nutr Food Res 2008.

1988. Pan MH, Ghai G and Ho CT: Food bioactives apoptosis, and cancer. Mol Nutr Food Res 2008, 52:43-52.

1989. Ramos S: Cancer chemoprevention and chemotherapy: dietary polyphenols and signalling pathways. Mol Nutr Food Res 2008, 52:507-26.

1990. Youn HS, Lim HJ, Lee HJ, Hwang D, Yang M, Jeon R and Ryu JH: Garlic (Allium sativum) extract inhibits lipopolysaccharideinduced Toll-like receptor 4 dimerization. Biosci Biotechnol Biochem 2008, 72:368-75.

1991. Dower SK and Qwarnstrom EE: Signalling networks, inflammation and innate immunity. Biochem Soc Trans 2003, 3 I: |462-7|.

1992. Leonard SS, Harris GK and Shi XL: Metal-induced oxidative stress and signal transduction. Free Rad Biol Med 2004, 37:1921-1942.

1993. Matsuzawa A, Saegusa K, Noguchi T, Sadamitsu C, Nishitoh H, Nagai S, Koyasu S, Matsumoto K, Takeda $\mathrm{K}$ and Ichijo H: ROSdependent activation of the TRAF6-ASKI-p38 pathway is selectively required for TLR4-mediated innate immunity. Nat Immunol 2005, 6:587-92.

1994. Schreck R, Rieber P and Baeuerle PA: Reactive oxygen intermediates as apparently widely used messengers in the activation of the NF-Kappa-B transcription Factor and HIV-I. EMBO J I99I, I 0:2247-2258.

1995. Schreck R, Albermann K and Baeuerle PA: Nuclear factor kappa$B$ - an oxidative stress-responsive transcription factor of eukaryotic cells (a Review). Free Rad Res Comm 1992, I 7:221-237.

1996. Schreck R, Meier B, Mannel DN, Droge W and Baeuerle PA: Dithiocarbamates as potent inhibitors of nuclear factor kappa B activation in intact cells. J Exp Med 1992 175:1 181-94.

1997. Mohan N, Sadeghi K, Reiter RJ and Meltz ML: The neurohormone melatonin inhibits cytokine, mitogen and ionizing radiation induced NF-kappa B. Biochemistry and Molecular Biology International 1995, 37:1063-1070.

1998. Baeuerle PA and Baltimore D: NF-kappa B: ten years after. Cell 1996, 87:I3-20.

1999. Baldwin AS Jr: The NF-kappa B and I kappa B proteins: new discoveries and insights. Annu Rev Immunol 1996, I 4:649-83.

2000. Dalton TP, Shertzer HG and Puga A: Regulation of gene expression by reactive oxygen. Annu Rev Pharmacol Toxicol 1999, 39:67-I0I.

200 I. Li C, Browder W and Kao RL: Early activation of transcription factor NF-kappaB during ischemia in perfused rat heart. Am J Physiol 1999, 276:H543-52.

2002. Wang S, Leonard SS, Castranova V, Vallyathan V and Shi X: The role of superoxide radical in TNF-alpha induced NFkappaB activation. Ann Clin Lab Sci 1999, 29:192-9.

2003. Knight JA: Review: Free radicals, antioxidants, and the immune system. Ann Clin Lab Sci 2000, 30:145-158.

2004. Schoonbroodt $S$ and Piette J: Oxidative stress interference with the nuclear factor-kappa $B$ activation pathways. Biochem Pharmacol 2000, 60:1075-83.

2005. Forman $H$ J and Torres $M$ : Reactive oxygen species and cell signaling: respiratory burst in macrophage signaling. $\mathrm{Am} J$ Respir Crit Care Med 2002, I66:S4-8.

2006. Wang SW, Kotamraju S, Konorev E, Kalivendi S, Joseph J and Kalyanaraman B: Activation of nuclear factor-kappa B during doxorubicin-induced apoptosis in endothelial cells and myocytes is pro-apoptotic: the role of hydrogen peroxide. Biochemical Journal 2002, 367:729-740.

2007. Haddad J]: Science review: Redox and oxygen-sensitive transcription factors in the regulation of oxidant-mediated lung injury: role for nuclear factor-kappaB. Crit Care 2002 6:48|-90.

2008. Asehnoune K, Strassheim D, Mitra S, Kim JY and Abraham E: Involvement of reactive oxygen species in Toll-like receptor 4-dependent activation of NF-kappa B. J Immunol 2004, I 72:2522-9.

2009. Poli G, Leonarduzzi G, Biasi F and Chiarpotto E: Oxidative stress and cell signalling. Curr Med Chem 2004, I I: I I63-I I82.

2010. Manna SK, Sarkar S, Barr J. Wise K, Barrera EV, Jejelowo O, RiceFicht $A C$ and Ramesh GT: Single-walled carbon nanotube induces oxidative stress and activates nuclear transcription
factor-kappaB in human keratinocytes. Nano Lett 2005, 5: 1676-84.

20II. Bubici C, Papa S, Dean K and Franzoso G: Mutual cross-talk between reactive oxygen species and nuclear factor-kappa B: molecular basis and biological significance. Oncogene 2006, 25:6731-48

2012. Bubici C, Papa S, Pham CG, Zazzeroni F and Franzoso G: The NFkappaB-mediated control of ROS and JNK signaling. Histol Histopathol 2006, 21:69-80.

2013. Gloire G, Legrand-Poels $S$ and Piette J: NF-kappaB activation by reactive oxygen species: fifteen years later. Biochem Pharmacol 2006, 72: | 493-505.

2014. Kim JY, Jung KJ, Choi JS and Chung HY: Modulation of the agerelated nuclear factor-kappaB (NF-kappaB) pathway by hesperetin. Aging Cell 2006, 5:40 I-II.

2015. Li LX and Frei B: Iron chelation inhibits NF-kappa Bmediated adhesion molecule expression by inhibiting p22 (phox) protein expression and NADPH oxidase activity. Arteriosclerosis Thromb Vasc Biol 2006, 26:2638-2643.

20I6. Kramer HF and Goodyear LJ: Exercise MAPK, and NF-kappaB signaling in skeletal muscle. J Appl Physiol 2007, I 03:388-95.

2017. Glauert HP: Vitamin E and NF-kappaB activation: a review. Vitam Horm 2007, 76:135-53.

2018. Perkins ND: Integrating cell-signalling pathways with NFkappaB and IKK function. Nat Rev Mol Cell Biol 2007, 8:49-62.

2019. Uwe S: Anti-inflammatory interventions of NF-kappaB signaling: potential applications and risks. Biochem Pharmacol 2008, 75: I567-79.

2020. Ghosh S and Karin M: Missing pieces in the NF-kappaB puzzle. Cell 2002, I09(Suppl):S8I-96.

2021. Hayden MS and Ghosh S: Signaling to NF-kappaB. Genes Dev 2004, 18:2195-224

2022 Nelson DE, lhekwaba AEC, Elliott M, Gibney CA, Foreman BE, Nelson G, See V, Horton CA, Spiller DG, Edwards SW, McDowell HP, Unitt JF, Sullivan E, Grimley R, Benson N, Broomhead DS, Kell DB and White MRH: Oscillations in NF- $\kappa$ $B$ signalling control the dynamics of target gene expression. Science 2004, 306:704-708.

2023. Hoffmann A and Baltimore D: Circuitry of nuclear factor kappaB signaling. Immunol Rev 2006, 2 I 0: |7|-86.

2024. Perkins ND and Gilmore TD: Good cop, bad cop: the different faces of NF-kappaB. Cell Death Differ 2006, I 3:759-72.

2025. Chen CC, Wang JK and Lin SB: Antisense oligonucleotides targeting protein kinase C-alpha, -beta I, or -delta but not eta inhibit lipopolysaccharide-induced nitric oxide synthase expression in RAW 264.7 macrophages: involvement of a nuclear factor kappa B-dependent mechanism. I Immunol 1998, I61:6206-14.

2026. Sanlioglu S, Williams CM, Samavati L, Butler NS, Wang G, McCray PB, Ritchie TC Jr, Hunninghake GW, Zandi $E$ and Engelhardt JF: Lipopolysaccharide induces Racl-dependent reactive oxygen species formation and coordinates tumor necrosis factor-alpha secretion through IKK regulation of NF-kappa B. J Biol Chem 200I, 276:30188-98.

2027. Hsu HY and Wen MH: Lipopolysaccharide-mediated reactive oxygen species and signal transduction in the regulation of interleukin-I gene expression. I Biol Chem 2002, 277:22 I3 I-9.

2028. Macdonald J, Galley HF and Webster NR: Oxidative stress and gene expression in sepsis. Br J Anaesth 2003, 90:22I-32.

2029. Park HS, Jung HY, Park EY, Kim J, Lee WJ and Bae YS: Cutting edge: direct interaction of TLR4 with NAD(P)H oxidase 4 isozyme is essential for lipopolysaccharide-induced production of reactive oxygen species and activation of NF-kappa B. I Immunol 2004, 173:3589-93.

2030. Pham CG, Bubici C, Zazzeroni F, Papa S, Jones J, Alvarez K Jayawardena S, De Smaele E, Cong R, Beaumont C, Torti FM, Torti SV and Franzoso G: Ferritin heavy chain upregulation by NF-kappaB inhibits TNFalpha-induced apoptosis by suppressing reactive oxygen species. Cell 2004, I | 9:529-42.

203I. Allan SM, Tyrrell PJ and Rothwell NJ: Interleukin-I and neuronal injury. Nat Rev Immunol 2005, 5:629-40.

2032. Ihekwaba AEC, Broomhead DS, Grimley R, Benson N and Kell DB: Sensitivity analysis of parameters controlling oscillatory signalling in the NF- $\kappa$ B pathway: the roles of IKK and $I \kappa B \alpha$. Systems Biology 2004, 1:93-103.

2033. Yue H, Brown M, Knowles J, Wang H, Broomhead DS and Kell DB: Insights into the behaviour of systems biology models from dynamic sensitivity and identifiability analysis: a case study of an NF-kappaB signalling pathway. Mol Biosyst 2006, 2:640-649. 
2034. Ihekwaba AEC, Broomhead DS, Grimley R, Benson N, White MRH and Kell DB: Synergistic control of oscillations in the NF- $\kappa$ B signalling pathway. IEE Systems Biology 2005, I 52: I53-I60.

2035. Mignotte B and Vayssiere JL: Mitochondria and apoptosis. Eur J Biochem 1998, 252:1-15.

2036. Fleury C, Mignotte B and Vayssiere JL: Mitochondrial reactive oxygen species in cell death signaling. Biochimie 2002, 84: $|3|-4 \mid$.

2037. Bharti AC and Aggarwal BB: Nuclear factor-kappa B and cancer: its role in prevention and therapy. Biochem Pharmacol 2002, 64:883-8.

2038. Richmond A: NF- $\kappa$, chemokine gene transcription and tumour growth. Nat Rev Immunol 2002, 2:664-74.

2039. Zhang $Z$ and Rigas B: NF-kappaB, inflammation and pancreatic carcinogenesis: NF-kappaB as a chemoprevention target (review). Int I Oncol 2006, 29:185-92.

2040. Calzado MA, Bacher S and Schmitz ML: NF-kappaB inhibitors for the treatment of inflammatory diseases and cancer. Curr Med Chem 2007, 14:367-76.

204I. Roman-Blas JA and Jimenez SA: NF-kappaB as a potential therapeutic target in osteoarthritis and rheumatoid arthritis. Osteoarthritis Cartilage 2006, | 4:839-48.

2042. Saklatvala J: Inflammatory signaling in cartilage: MAPK and NF-kappaB pathways in chondrocytes and the use of inhibitors for research into pathogenesis and therapy of osteoarthritis. Curr Drug Targets 2007, 8:305-13.

2043. Simmonds RE and Foxwell BM: NF-\{kappa\}B and its relevance to arthritis and inflammation. Rheumatology (Oxford) 2008

2044. Smahi A Courtois G, Rabia SH, Doffinger R, Bodemer $C$ Munnich A, Casanova JL and Israel A: The NF-kappaB signalling pathway in human diseases: from incontinentia pigmenti to ectodermal dysplasias and immune-deficiency syndromes. Hum Mol Genet 2002, I I:237 I-5.

2045. Lezoualc'h F, Sparapani $M$ and Behl C: $\mathbf{N}$-acetyl-serotonin (normelatonin) and melatonin protect neurons against oxidative challenges and suppress the activity of the transcription factor NF-kappaB. J Pineal Res 1998, 24: 168-78.

2046. Post A, Holsboer $F$ and Behl C: Induction of NF-kappaB activity during haloperidol-induced oxidative toxicity in clonal hippocampal cells: suppression of NF-kappaB and neuroprotection by antioxidants. J Neurosci 1998, I 8:8236-46.

2047. Herrera J, Nava M, Romero F and Rodriguez-Iturbe B: Melatonin prevents oxidative stress resulting from iron and erythropoietin administration. Am J Kidney Dis 2001, 37:750-7.

2048. Beni SM, Kohen R, Reiter RJ, Tan DX and Shohami E: Melatonininduced neuroprotection after closed head injury is associated with increased brain antioxidants and attenuated late-phase activation of NF-kappaB and AP-I. FASEB J 2004, I 8: |49-5|.

2049. Rodriguez MI, Escames G, Lopez LC, Lopez A, Garcia JA, Ortiz F and Acuna-Castroviejo D: Chronic melatonin treatment reduces the age-dependent inflammatory process in senescence-accelerated mice. J Pineal Res 2007, 42:272-9.

2050. Takada Y, Mukhopadhyay A, Kundu GC, Mahabeleshwar GH Singh $S$ and Aggarwal BB: Hydrogen peroxide activates NFkappa B through tyrosine phosphorylation of I kappa B alpha and serine phosphorylation of p65: evidence for the involvement of I kappa B alpha kinase and Syk proteintyrosine kinase. J Biol Chem 2003, 278:24233-4I.

205I. Hsieh CC and Papaconstantinou J: Thioredoxin-ASK I complex levels regulate ROS-mediated p38 MAPK pathway activity in livers of aged and long-lived Snell dwarf mice. FASEB J 2006, 20:259-68.

2052. Walter DH, Dimmeler S and Zeiher AM: Effects of statins on endothelium and endothelial progenitor cell recruitment. Semin Vasc Med 2004, 4:385-93.

2053. Madonna R, Di Napoli P, Massaro M, Grilli A, Felaco M, De Caterina A, Tang D, De Caterina R and Geng YJ: Simvastatin attenuates expression of cytokine-inducible nitric-oxide synthase in embryonic cardiac myoblasts. J Biol Chem 2005 , 280: |3503-II.

2054. Urbich C and Dimmeler S: Risk factors for coronary artery disease, circulating endothelial progenitor cells, and the role of HMG-CoA reductase inhibitors. Kidney Int 2005, 67: $1672-6$.

2055. Gilmore TD and Herscovitch M: Inhibitors of NF-kappaB signaling: 785 and counting. Oncogene 2006, 25:6887-99.

2056. Lazzerini PE, Lorenzini S, Selvi E, Capecchi PL, Chindamo D, Bisogno S, Ghittoni R, Natale MR, Caporali F, Giuntini S, Marcolongo R, Galeazzi $M$ and Laghi-Pasini F: Simvastatin inhibits cytokine production and nuclear factor-kB activation in interleukin Ibeta-stimulated synoviocytes from rheumatoid arthritis patients. Clin Exp Rheumatol 2007, 25:696-700.

2057. Lee JY, Kim JS, Kim JM, Kim N, Jung HC and Song IS: Simvastatin inhibits NF-kappaB signaling in intestinal epithelial cells and ameliorates acute murine colitis. Int Immunopharmacol 2007, 7:24I-8.

2058. Lin SJ, Chen YH, Lin FY, Hsieh LY, Wang SH, Lin CY, Wang YC, $\mathrm{Ku} \mathrm{HH}$, Chen JW and Chen YL: Pravastatin induces thrombomodulin expression in TNFalpha-treated human aortic endothelial cells by inhibiting Racl and Cdc42 translocation and activity. J Cell Biochem 2007, I 0 I:642-53.

2059. Dolga AM, Nijholt IM, Ostroveanu A, Ten Bosch Q, Luiten PG and Eisel UL: Lovastatin induces neuroprotection through tumor necrosis factor receptor 2 signaling pathways. J Alzheimers Dis 2008, I3:I I I-22.

2060. Gao F, Linhartova L, Johnston AM and Thickett DR: Statins and sepsis. British Journal of Anaesthesia 2008, 100:288-298.

206I. Galter D, Mihm S and Dröge W: Distinct effects of glutathione disulphide on the nuclear transcription factor kappa $B$ and the activator protein-I. Eur J Biochem 1994, 22 I:639-48.

2062. Kretz-Remy C, Mehlen P, Mirault ME and Arrigo AP: Inhibition of I kappa B-alpha phosphorylation and degradation and subsequent NF-kappa B activation by glutathione peroxidase overexpression. J Cell Biol 1996, I 33:I083-93.

2063. García-Ruiz C, Colell A, Morales A, Kaplowitz N and FernándezCheca IC: Role of oxidative stress generated from the mitochondrial electron transport chain and mitochondrial glutathione status in loss of mitochondrial function and activation of transcription factor nuclear factor-kappa B: studies with isolated mitochondria and rat hepatocytes. Mol Pharmacol 1995, 48:825-34

2064. Mihm S, Galter D and Dröge W: Modulation of transcription factor NF kappa B activity by intracellular glutathione levels and by variations of the extracellular cysteine supply. Faseb J 1995, 9:246-52.

2065. Ginn-Pease ME and Whisler RL: Optimal NF kappa B mediated transcriptional responses in Jurkat $T$ cells exposed to oxidative stress are dependent on intracellular glutathione and costimulatory signals. Biochem Biophys Res Commun 1996, 226:695-702

2066. Pinkus R, Weiner LM and Daniel V: Role of oxidants and antioxidants in the induction of AP-I, NF-kappaB, and glutathione S-transferase gene expression. J Biol Chem I996, 27 I: | 3422-9.

2067. Brigelius-Flohé R, Friedrichs B, Maurer S, Schultz M and Streicher $R$ Interleukin-I-induced nuclear factor kappa $B$ activation is inhibited by overexpression of phospholipid hydroperoxide glutathione peroxidase in a human endothelial cell line. Biochem J I997, 328(Pt I): |99-203.

2068. Sen CK, Khanna S, Reznick AZ, Roy S and Packer L: Glutathione regulation of tumor necrosis factor-alpha-induced NFkappa B activation in skeletal muscle-derived L6 cells. Biochem Biophys Res Commun 1997, 237:645-9.

2069. Cho S, Urata Y, lida T, Goto S, Yamaguchi M, Sumikawa K and Kondo T: Glutathione downregulates the phosphorylation of I kappa B: autoloop regulation of the NF-kappa Bmediated expression of NF-kappa B subunits by TNF-alpha in mouse vascular endothelial cells. Biochem Biophys Res Commun 1998, 253:104-8.

2070. Rokutan K, Teshima S, Miyoshi M, Kawai T, Nikawa T and Kishi K: Glutathione depletion inhibits oxidant-induced activation of nuclear factor-kappa B, AP-I, and c-Jun/ATF-2 in cultured guinea-pig gastric epithelial cells. J Gastroenterol 1998, 33:646-55.

207I. Fernandez PC, Machado J Jr, Heussler VT, Botteron C, Palmer GH and Dobbelaere DA: The inhibition of NF-kappaB activation pathways and the induction of apoptosis by dithiocarbamates in $\mathrm{T}$ cells are blocked by the glutathione precursor N-acetyl-L-cysteine. Biol Chem 1999, 380:I383-94.

2072. Haddad J, Olver RE and Land SC: Antioxidant/pro-oxidant equilibrium regulates HIF-Ialpha and NF-kappa B redox sensitivity. Evidence for inhibition by glutathione oxidation in alveolar epithelial cells. J Biol Chem 2000, 275:2 I I30-9.

2073. Haddad JJ and Land SC: $\mathbf{O}_{2}$-evoked regulation of HIF-Ialpha and NF-kappaB in perinatal lung epithelium requires glutathione biosynthesis. Am J Physiol Lung Cell Mol Physiol 2000, 278:L492-503. 
2074. Rahman I and MacNee W: Oxidative stress and regulation of glutathione in lung inflammation. Eur Respir J 2000, I 6:534-54.

2075. Rahman I and MacNee W: Regulation of redox glutathione levels and gene transcription in lung inflammation: therapeutic approaches. Free Radic Biol Med 2000, 28: I405-20.

2076. Davis W Jr, Ronai Z and Tew KD: Cellular thiols and reactive oxygen species in drug-induced apoptosis. J Pharmacol Exp Ther 200I, 296: I-6.

2077. Nishi T, Shimizu N, Hiramoto M, Sato I, Yamaguchi Y, Hasegawa M, Aizawa S, Tanaka H, Kataoka K, Watanabe $H$ and Handa H: Spatial redox regulation of a critical cysteine residue of NF-kappa B in vivo. I Biol Chem 2002, 277:44548-56.

2078. Biswas SK, McClure D, Jimenez LA, Megson IL and Rahman I: Curcumin induces glutathione biosynthesis and inhibits NF-kappaB activation and interleukin- 8 release in alveolar epithelial cells: mechanism of free radical scavenging activity. Antioxid Redox Signal 2005, 7:32-4I.

2079. Biswas SK, Newby DE, Rahman I and Megson IL: Depressed glutathione synthesis precedes oxidative stress and atherogenesis in Apo-E(-/-) mice. Biochem Biophys Res Commun 2005, 338: 1368-73.

2080. Rahman I, Biswas SK, Jimenez LA, Torres $M$ and Forman HJ: Glutathione, stress responses, and redox signaling in lung inflammation. Antioxid Redox Signal 2005, 7:42-59.

208I. Cuzzocrea S, Chatterjee PK, Mazzon E, Dugo L, Serraino I, Britti D, Mazzullo G, Caputi AP and Thiemermann C: Pyrrolidine dithiocarbamate attenuates the development of acute and chronic inflammation. Br J Pharmacol 2002, I35:496-5 I0.

2082. Desoize $B$ : Metals and metal compounds in carcinogenesis. In Vivo 2003, I 7:529-539.

2083. Xiong SG, She HY, Takeuchi H, Han B, Engelhardt JF, Barton $\mathrm{CH}$, Zandi $E$, Giulivi $C$ and Tsukamoto $\mathrm{H}$ : Signaling role of intracellular iron in NF-kappa B activation. Journal of Biological Chemistry 2003, 278: 17646-17654.

2084. Parodi FE, Mao D, Ennis TL, Bartoli MA and Thompson RW: Suppression of experimental abdominal aortic aneurysms in mice by treatment with pyrrolidine dithiocarbamate, an antioxidant inhibitor of nuclear factor-kappaB. J Vasc Surg 2005, 41:479-89.

2085. Antosiewicz J, Ziolkowski W, Kaczor JJ and HermanAntosiewicz A: Tumor necrosis factor-alpha-induced reactive oxygen species formation is mediated by JNKIdependent ferritin degradation and elevation of labile iron pool. Free Radic Biol Med 2007, 43:265-70.

2086. Vélez-Pardo C, Ospina GG and Del Río MJ: Abeta[25-35] peptide and iron promote apoptosis in lymphocytes by an oxidative stress mechanism: involvement of $\mathrm{H}_{2} \mathrm{O}_{2}$, caspase3, NF-kappaB, p53 and c-Jun. Neurotoxicology 2002, 23:35|-65.

2087. Bisti $S$ and Soteriadou K: Is the reactive oxygen speciesdependent-NF-kappaB activation observed in iron-loaded BALB/c mice a key process preventing growth of Leishmania major progeny and tissue-damage? Microbes Infect 2006, 8: $1473-82$

2088. Bu DX, Hemdahl AL, Gabrielsen A, Fuxe J, Zhu C, Eriksson P and Yan ZQ: Induction of neutrophil gelatinase-associated lipocalin in vascular injury via activation of nuclear factorkappaB. Am J Pathol 2006, I 69:2245-53.

2089. Sommer G, Weise S, Kralisch S, Lossner U, Bluher M, Stumvoll M and Fasshauer M: Lipocalin-2 is induced by interleukin-Ibeta in murine adipocytes in vitro. / Cell Biochem 2008.

2090. Lee P, Peng H, Gelbart T, Wang L and Beutler E: Regulation of hepcidin transcription by interleukin-I and interleukin-6. Proc Natl Acad Sci USA 2005, 102:1906-10.

209I. Duvigneau IC, Piskernik C, Haindl S, Kloesch B, Hartl RT, Huttemann M, Lee I, Ebel T, Moldzio R, Gemeiner M, Redl H and Kozlov AV: A novel endotoxin-induced pathway: upregulation of heme oxygenase $I$, accumulation of free iron, and free iron-mediated mitochondrial dysfunction. Lab Invest 2008, 88:70-7.

2092. Whitcomb DC, Aoun E, Vodovotz Y, Clermont G and Barmada MM: Evaluating disorders with a complex genetics basis. the future roles of meta-analysis and systems biology. Dig Dis Sci 2005, 50:2195-202.

2093. Xiong M, Feghali-Bostwick CA, Arnett FC and Zhou X: A systems biology approach to genetic studies of complex diseases. FEBS Lett 2005, 579:5325-32.

2094. Ideker T, Galitski T and Hood L: A new approach to decoding life: systems biology. Annu Rev Genomics Hum Genet 2001, 2:343-72.
2095. Barabási A-L and Oltvai ZN: Network biology: understanding the cell's functional organization. Nat Rev Genet 2004, 5: $101-113$

2096. Morel NM, Holland JM, Greef van der J, Marple EW, Clish C Loscalzo J and Naylor S: Primer on medical genomics. Part XIV: Introduction to systems biology-a new approach to understanding disease and treatment. Mayo Clin Proc 2004, 79:65I-8.

2097. Khalil IG and Hill C: Systems biology for cancer. Curr Opin Oncol 2005, I 7:44-8.

2098. Moore JH, Boczko EM and Summar ML: Connecting the dots between genes biochemistry, and disease susceptibility: systems biology modeling in human genetics. Mol Genet Metab 2005, 84:104-II.

2099. Hornberg J], Bruggeman FJ, Westerhoff HV and Lankelma J: Cancer: a Systems Biology disease. Biosystems 2006, 83:8I-90.

2100 . Lemberger T: Systems biology in human health and disease. Mol Syst Biol 2007, 3:136.

2I0I. Loscalzo J, Kohane I and Barabasi AL: Human disease classification in the postgenomic era: a complex systems approach to human pathobiology. Mol Syst Biol 2007, 3: 124.

2102. Schnackenberg LK and Beger RD: Monitoring the health to disease continuum with global metabolic profiling and systems biology. Pharmacogenomics 2006, 7:1077-86.

2103. Featherstone DE and Broadie K: Wrestling with pleiotropy: genomic and topological analysis of the yeast gene expression network. Bioessays 2002, 24:267-74.

2 104. Castrillo JI, Zeef LA, Hoyle DC, Zhang N, Hayes A, Gardner DC], Cornell MJ, Petty J, Hakes L, Wardleworth L, Rash B, Brown M, Dunn WB, Broadhurst D, O'Donoghue K, Hester SS, Dunkley TPJ Hart SR, Swainston N, Li P, Gaskell SJ, Paton NW, Lilley KS, Kell DB and Oliver SG: Growth control of the eukaryote cell: a systems biology study in yeast. J. Biol 2007, 6:4.

2105. Raamsdonk LM, Teusink B, Broadhurst D, Zhang $N$, Hayes $A$, Walsh M, Berden JA, Brindle KM, Kell DB, Rowland J], Westerhoff HV, van Dam K and Oliver SG: A functional genomics strategy that uses metabolome data to reveal the phenotype of silent mutations. Nature Biotechnol 200I, 19:45-50.

2106. Mo ML, Jamshidi $N$ and Palsson BO: A genome-scale, constraint-based approach to systems biology of human metabolism. Mol Biosyst 2007, 3:598-603.

2107. Kell DB: Metabolomics and systems biology: making sense of the soup. Curr Op Microbiol 2004, 7:296-307.

2108. Kell DB, Brown M, Davey HM, Dunn WB, Spasic I and Oliver SG: Metabolic footprinting and Systems Biology: the medium is the message. Nat Rev Microbiol 2005, 3:557-565.

2109. Kell DB: Metabolomics, modelling and machine learning in systems biology: towards an understanding of the languages of cells. The 2005 Theodor Bücher lecture. FEBS J 2006, 273:873-894.

2110. Kell DB: Systems biology, metabolic modelling and metabolomics in drug discovery and development. Drug Disc Today 2006, II:1085-1092.

21।I. Kell DB: Metabolomic biomarkers: search, discovery and validation. Exp Rev Mol Diagnost 2007, 7:329-333.

2112. Greef van der J, Martin S, Juhasz P, Adourian A, Plasterer T, Verheij ER and McBurney RN: The art and practice of systems biology in medicine: mapping patterns of relationships. J Proteome Res 2007, 6: I540-59.

2113. Kacser $\mathrm{H}$ and Burns JA: The control of flux. Rate Control of Biological Processes. Symposium of the Society for Experimental Biology Cambridge University Press, Cambridge: Davies DD 1973 , 27:65-104.

2II4. Heinrich R and Rapoport TA: A linear steady-state treatment of enzymatic chains. General properties, control and effector strength. Eur J Biochem 1974, 42:89-95.

2II5. Kell DB and Westerhoff HV: Metabolic control theory: its role in microbiology and biotechnology. FEMS Microbiol Rev 1986, 39:305-320.

2116. Westerhoff HV and van Dam K: Thermodynamics and control of biological free energy transduction Elsevier, Amsterdam; 1987.

2117. Westerhoff HV and Kell DB: Matrix method for determining the steps most rate-limiting to metabolic fluxes in biotechnological processes. Biotechnol Bioeng I987, 30:101-107.

2II8. Fell DA: Understanding the control of metabolism Portland Press, London; 1996.

2119. Heinrich $R$ and Schuster S: The regulation of cellular systems Chapman \& Hall, New York; 1996. 
2120. Murphy MP and Partridge L: Toward a control theory analysis of aging. Annu Rev Biochem 2008, 77:777-98.

2121 . Morris JG: The physiology of obligate anaerobiosis. Adv Microb Physiol 1975, I2:169-246.

2122. Kirkwood TB, Boys RJ, Gillespie CS, Proctor Cl, Shanley DP and Wilkinson DJ: Towards an e-biology of ageing: integrating theory and data. Nat Rev Mol Cell Biol 2003, 4:243-9.

2123. Hofmeyr $\mathrm{JH}$ and Westerhoff HV: Building the cellular puzzle: control in multi-level reaction networks. I Theor Biol 2001, 208:26I-85.

2 I24. Saltelli A, Tarantola S, Campolongo F and Ratto M: Sensitivity analysis in practice: a guide to assessing scientific models Wiley, New York; 2004.

2125. Sauro HM and Kholodenko BN: Quantitative analysis of signaling networks. Prog Biophys Mol Biol 2004, 86:5-43.

2126. Lüdtke N, Panzeri S, Brown M, Broomhead DS, Knowles J, Montemurro MA and Kell DB: Information-theoretic Sensitivity Analysis: a general method for credit assignment in complex networks. J Roy Soc Interface 2008, 5:223-235.

2127. Saltelli A, Ratto M, Andres T, Campolongo F, Cariboni J, Gatelli D, Saisana $M$ and Tarantola S: Global sensitivity analysis: the primer WileyBlackwell, New York; 2008.

2128. Smallbone K, Simeonidis E, Broomhead DS and Kell DB: Something from nothing: bridging the gap between constraintbased and kinetic modelling. FEBS j 2007, 274:5576-5585.

2129. Mendes $P$ and Kell DB: Non-linear optimization of biochemical pathways: applications to metabolic engineering and parameter estimation. Bioinformatics 1998, 14:869-883.

2130. Moles CG, Mendes P and Banga JR: Parameter estimation in biochemical pathways: a comparison of global optimization methods. Genome Res 2003, I 3:2467-74.

2131. Rodriguez-Fernandez M, Mendes P and Banga JR: A hybrid approach for efficient and robust parameter estimation in biochemical pathways. Biosystems 2006, 83:248-65.

2132. Wilkinson SJ, Benson N and Kell DB: Proximate parameter tuning for biochemical networks with uncertain kinetic parameters. Mol Biosyst 2008, 4:74-97.

2133. Klipp E, Herwig R, Kowald A, Wierling C and Lehrach H: Systems biology in practice: concepts, implementation and clinical application Wiley/VCH, Berlin; 2005.

2 134. Alon U: An introduction to systems biology: design principles of biological circuits Chapman and Hall/CRC, London; 2006.

2135. Palsson BØ: Systems biology: properties of reconstructed networks Cambridge University Press, Cambridge; 2006.

2136. Doyle FJ 3rd and Stelling J: Systems interface biology. J R Soc Interface 2006, 3:603-16.

2137. Noble D: The music of life: biology beyond genes Oxford University Press, Oxford; 2006

2138. Hopkins AL: Network pharmacology: the next paradigm in drug discovery. Nat Chem Biol 2008, 4:682-690.

2139. Rand DA: Mapping global sensitivity of cellular network dynamics: sensitivity heat maps and a global summation law. J R Soc Interface 2008, 5(SuppI I):S59-69.

2140. Tagkopoulos I, Liu YC and Tavazoie S: Predictive behavior within microbial genetic networks. Science 2008, 320: I3 I3-7.

214I. Muscoli C, Cuzzocrea S, Riley DP, Zweier JL, Thiemermann C Wang ZQ and Salvemini D: On the selectivity of superoxide dismutase mimetics and its importance in pharmacological studies. British Journal of Pharmacology 2003, I40:445-460.

2142. Fell DA: Increasing the flux in metabolic pathways: A metabolic control analysis perspective. Biotechnol Bioeng 1998, 58: $121-124$

2143. Sai $Y$ and Tsuji A: Transporter-mediated drug delivery: recent progress and experimental approaches. Drug Discov Today 2004, 9:712-20.

2144. Schulze MB, Manson JE, Willett WC and Hu FB: Processed meat intake and incidence of Type 2 diabetes in younger and middle-aged women. Diabetologia 2003, 46:|465-73.

2 145. Miyake T, Kung CK and Goldwasser E: Purification of human erythropoietin. J Biol Chem I977, 252:5558-64.

2146. Jacobs K, Shoemaker C, Rudersdorf R, Neill SD, Kaufman RJ, Mufson A, Seehra J, Jones SS, Hewick R and Fritsch EF, et al: Isolation and characterization of genomic and CDNA clones of human erythropoietin. Nature 1985, 3 I3:806-10.

2147. Lin FK, Suggs S, Lin CH, Browne JK, Smalling R, Egrie JC, Chen KK, Fox GM, Martin F and Stabinsky Z, et al: Cloning and expression of the human erythropoietin gene. Proc Natl Acad Sci USA I 985, 82:7580-4

2148. Fenjves ES, Ochoa MS, Cabrera O, Mendez AJ, Kenyon NS, Inverardi $L$ and Ricordi $C$ : Human, nonhuman primate, and rat pancreatic islets express erythropoietin receptors. Transplantation 2003, 75: I356-60.

2149. Ghezzi P and Brines M: Erythropoietin as an antiapoptotic, tissue-protective cytokine. Cell Death Differ 2004, I I (SuppI I): S37-44.

2150. Brines $M$ and Cerami A: Emerging biological roles for erythropoietin in the nervous system. Nat Rev Neurosci 2005, 6:484-94.

215I. Chong ZZ, Li F and Maiese K: Oxidative stress in the brain: novel cellular targets that govern survival during neurodegenerative disease. Prog Neurobiol 2005, 75:207-46.

2152. Brines $M$ and Cerami $A$ : Discovering erythropoietin's extrahematopoietic functions: biology and clinical promise. Kidney Int 2006, 70:246-50.

2153. Coleman TR, Westenfelder C, Togel FE, Yang Y, Hu Z, Swenson L, Leuvenink HG, Ploeg RJ, d'Uscio LV, Katusic ZS, Ghezzi P, Zanetti A, Kaushansky K, Fox NE, Cerami A and Brines M: Cytoprotective doses of erythropoietin or carbamylated erythropoietin have markedly different procoagulant and vasoactive activities. Proc Natl Acad Sci USA 2006, I 03:5965-70.

2154. Ghezzi $P$ and Mengozzi $M$ : Activities of erythropoietin on tumors: an immunological perspective. Eur J Immunol 2007, 37:1427-30.

2155. Tönges L, Schlachetzki JCM, Weishaupt JH and Bähr M: Hematopoietic cytokines - on the verge of conquering neurology. Current Molecular Medicine 2007, 7:I57-I70.

2156. Cai Z, Manalo DJ, Wei G, Rodriguez ER, Fox-Talbot K, Lu H, Zweier JL and Semenza GL: Hearts from rodents exposed to intermittent hypoxia or erythropoietin are protected against ischemia-reperfusion injury. Circulation 2003, I 08:79-85.

2157. Tramontano AF, Muniyappa R, Black AD, Blendea MC, Cohen I, Deng L, Sowers JR, Cutaia MV and El-Sherif N: Erythropoietin protects cardiac myocytes from hypoxia-induced apoptosis through an Akt-dependent pathway. Biochem Biophys Res Commun 2003, 308:990-4.

2158. Bogoyevitch MA: An update on the cardiac effects of erythropoietin cardioprotection by erythropoietin and the lessons learnt from studies in neuroprotection. Cardiovasc $\operatorname{Res} 2004$, 63:208-16.

2159. Cai Z and Semenza GL: Phosphatidylinositol-3-kinase signaling is required for erythropoietin-mediated acute protection against myocardial ischemia/reperfusion injury. Circulation 2004, 109:2050-3.

2160. Wright GL, Hanlon P, Amin K, Steenbergen C, Murphy E and Arcasoy MO: Erythropoietin receptor expression in adult rat cardiomyocytes is associated with an acute cardioprotective effect for recombinant erythropoietin during ischemiareperfusion injury. FASEB J 2004, I 8:103|-3.

216I. Fiordaliso F, Chimenti S, Staszewsky L, Bai A, Carlo E, Cuccovillo I, Doni M, Mengozzi M, Tonelli R, Ghezzi P, Coleman T, Brines $M$, Cerami $A$ and Latini R: A nonerythropoietic derivative of erythropoietin protects the myocardium from ischemiareperfusion injury. Proc Natl Acad Sci USA 2005, I 02:2046-5I.

2162. Moon C, Krawczyk M, Paik D, Lakatta EG and Talan MI: Cardioprotection by recombinant human erythropoietin following acute experimental myocardial infarction: dose response and therapeutic window. Cardiovasc Drugs Ther 2005, 19:243-50.

2163. Rui T, Feng Q, Lei $M$, Peng $T$, Zhang J, Xu M, Abel ED Xenocostas A and Kvietys PR: Erythropoietin prevents the acute myocardial inflammatory response induced by ischemia/reperfusion via induction of AP-I. Cardiovasc Res 2005, 65:719-27.

2164. Lee ST, Chu K, Sinn DI, Jung KH, Kim EH, Kim SJ, Kim JM, Ko SY, Kim $M$ and Roh JK: Erythropoietin reduces perihematomal inflammation and cell death with eNOS and STAT3 activations in experimental intracerebral hemorrhage. Neurochem 2006, 96:1728-39.

2165. Li Y, Takemura G, Okada H, Miyata S, Maruyama R, Li L, Higuchi M, Minatoguchi S, Fujiwara $T$ and Fujiwara $H$ : Reduction of inflammatory cytokine expression and oxidative damage by erythropoietin in chronic heart failure. Cardiovasc Res 2006, 71:684-94

2166. Li L, Takemura G, Li Y, Miyata S, Esaki M, Okada H, Kanamori H, Khai NC, Maruyama R, Ogino A, Minatoguchi S, Fujiwara T and Fujiwara $\mathrm{H}$ : Preventive effect of erythropoietin on cardiac dysfunction in doxorubicin-induced cardiomyopathy. Circulation 2006, I I 3:535-43. 
2167. Miki T, Miura T, Yano T, Takahashi A, Sakamoto J, Tanno M, Kobayashi H, lkeda $Y$, Nishihara M, Naitoh $K$, Ohori $K$ and Shimamoto K: Alteration in erythropoietin-induced cardioprotective signaling by postinfarct ventricular remodeling. J Pharmacol Exp Ther 2006, 3 I 7:68-75.

2168. Moon C, Krawczyk M, Paik D, Coleman T, Brines M, Juhaszova M, Sollott SJ, Lakatta EG and Talan MI: Erythropoietin, modified to not stimulate red blood cell production, retains its cardioprotective properties. J Pharmacol Exp Ther 2006, 3 | 6:999-1005.

2169. Nishihara M, Miura T, Miki T, Sakamoto J, Tanno M, Kobayashi H, Ikeda Y, Ohori K, Takahashi A and Shimamoto K: Erythropoietin affords additional cardioprotection to preconditioned hearts by enhanced phosphorylation of glycogen synthase kinase-3 beta. Am J Physiol Heart Circ Physiol 2006, 29 I:H748-55.

2170. Binbrek AS, Mittal B, Rao KN and Sobel BE: The potential of erythropoietin for conferring cardioprotection complementing reperfusion. Coron Artery Dis 2007, I 8:583-5.

2171. Chen X, Chen Y, Bi Y, Fu N, Shan C, Wang S, Aslam S, Wang PW and $\mathrm{Xu}$ J: Preventive cardioprotection of erythropoietin against doxorubicin-induced cardiomyopathy. Cardiovasc Drugs Ther 2007, 21:367-74.

2172. Gao E, Boucher M, Chuprun JK, Zhou RH, Eckhart AD and Koch WJ: Darbepoetin alfa, a long-acting erythropoietin analog, offers novel and delayed cardioprotection for the ischemic heart. Am J Physiol Heart Circ Physiol 2007, 293:H60-8.

2173. Putten van der K, Braam B, Jie KE and Gaillard CA: Mechanisms of Disease: erythropoietin resistance in patients with both heart and kidney failure. Nat Clin Pract Nephrol 2008, 4:47-57.

2174. Furlani D, Klopsch C, Gabel R, Ugurlucan M, Pittermann E, Klee D, Wagner K, Li W, Wang W, Ong LL, Nizze H, Titze U, Lutzow K, Lendlein A, Steinhoff $G$ and $M a N$ : Intracardiac erythropoietin injection reveals antiinflammatory potential and improved cardiac functions detected by Forced Swim Test. Transplant Proc 2008, 40:962-6.

2175. Robey TE, Saiget MK, Reinecke H and Murry CE: Systems approaches to preventing transplanted cell death in cardiac repair. I Mol Cell Cardiol 2008, 45:567-8I.

2176. Sirén $A L$ and Ehrenreich H: Erythropoietin-a novel concept for neuroprotection. Eur Arch Psychiatry Clin Neurosci 2001, 25 I: $179-84$.

2177. Buemi M, Cavallaro E, Floccari F, Sturiale A, Aloisi C, Trimarchi M, Grasso G, Corica F and Frisina N: Erythropoietin and the brain: from neurodevelopment to neuroprotection. Clin Sci (Lond) 2002, 103:275-82

2178. Junk AK, Mammis A, Savitz SI, Singh M, Roth S, Malhotra S, Rosenbaum PS, Cerami A, Brines $M$ and Rosenbaum DM: Erythropoietin administration protects retinal neurons from acute ischemia-reperfusion injury. Proc Natl Acad Sci USA 2002, 99:10659-64.

2179. Juul S: Erythropoietin in the central nervous system, and its use to prevent hypoxic-ischemic brain damage. Acta Paediatr Suppl 2002, 91:36-42.

2180. Ruscher K, Freyer D, Karsch M, Isaev N, Megow D, Sawitzki B, Priller J, Dirnagl $U$ and Meisel A: Erythropoietin is a paracrine mediator of ischemic tolerance in the brain: evidence from an in vitro model. J Neurosci 2002, 22:1029|-301.

2181. Campana WM and Myers RR: Exogenous erythropoietin protects against dorsal root ganglion apoptosis and pain following peripheral nerve injury. Eur $j$ Neurosci 2003, I 8: |497-506.

2182. Prass K, Scharff A, Ruscher K, Lowl D, Muselmann C, Victorov I, Kapinya K, Dirnagl $U$ and Meisel A: Hypoxia-induced stroke tolerance in the mouse is mediated by erythropoietin. Stroke 2003, 34: 1981-6.

2183. Villa $P$, Bigini $P$, Mennini T, Agnello $D$, Laragione $T$, Cagnotto $A$ Viviani B, Marinovich M, Cerami A, Coleman TR, Brines $M$ and Ghezzi P: Erythropoietin selectively attenuates cytokine production and inflammation in cerebral ischemia by targeting neuronal apoptosis. J Exp Med 2003, I98:97|-5.

2184. Digicaylioglu M, Garden G, Timberlake S, Fletcher L and Lipton SA: Acute neuroprotective synergy of erythropoietin and insulin-like growth factor I. Proc Natl Acad Sci USA 2004, | $01: 9855-60$.

2185. Ehrenreich $\mathrm{H}$, Aust C, Krampe H, Jahn H, Jacob S, Herrmann M and Siren AL: Erythropoietin: novel approaches to neuroprotection in human brain disease. Metab Brain Dis 2004, 19:195-206.

2186. Genc S, Koroglu TF and Genc K: Erythropoietin and the nervous system. Brain Res 2004, I000:19-31.
2187. Keswani SC, Buldanlioglu U, Fischer A, Reed N, Polley M, Liang H, Zhou C, Jack C, Leitz GJ and Hoke A: A novel endogenous erythropoietin mediated pathway prevents axonal degeneration. Ann Neurol 2004, 56:815-26.

2188. Lipton SA: Erythropoietin for neurologic protection and diabetic neuropathy. N Engl J Med 2004, 350:25 I6-7.

2 189. Marti HH: Erythropoietin and the hypoxic brain. J Exp Biol 2004, 207:3233-42.

2190. Prass K, Ruscher K, Karsch M, Isaev N, Megow D, Priller J, Scharff A, Dirnagl $U$ and Meisel A: Desferrioxamine induces delayed tolerance against cerebral ischemia in vivo and in vitro. I Cereb Blood Flow Metab 2002, 22:520-5.

2191. Diem R, Sättler MB, Merkler D, Demmer I, Maier K, Stadelmann C, Ehrenreich $\mathrm{H}$ and Bähr M: Combined therapy with methylprednisolone and erythropoietin in a model of multiple sclerosis. Brain 2005, 128:375-85.

2192. Yatsiv I, Grigoriadis N, Simeonidou C, Stahel PF, Schmidt OI, Alexandrovitch AG, Tsenter J and Shohami E: Erythropoietin is neuroprotective, improves functional recovery, and reduces neuronal apoptosis and inflammation in a rodent model of experimental closed head injury. FASEB J 2005, 19:1701-3.

2193. Savino C, Pedotti R, Baggi F, Ubiali F, Gallo B, Nava S, Bigini $P$ Barbera S, Fumagalli E, Mennini T, Vezzani A, Rizzi M, Coleman T, Cerami A, Brines M, Ghezzi $P$ and Bianchi R: Delayed administration of erythropoietin and its non-erythropoietic derivatives ameliorates chronic murine autoimmune encephalomyelitis. J Neuroimmunol 2006, I 72:27-37.

2194. Sirén AL, Radyushkin K, Boretius S, Kammer D, Riechers CC Natt O, Sargin D, Watanabe T, Sperling S, Michaelis T, Price J, Meyer B, Frahm J and Ehrenreich $\mathrm{H}$ : Global brain atrophy after unilateral parietal lesion and its prevention by erythropoietin. Brain 2006, I29:480-9.

2195. Chen G, Shi JX, Hang CH, Xie W, Liu J and Liu X: Inhibitory effect on cerebral inflammatory agents that accompany traumatic brain injury in a rat model: a potential neuroprotective mechanism of recombinant human erythropoietin (rhEPO). Neurosci Lett 2007, 425: I77-82.

2196. Cherian L, Goodman JC and Robertson C: Neuroprotection with erythropoietin administration following controlled cortical impact injury in rats. J Pharmacol Exp Ther 2007, 322:789-94

2197. Ehrenreich $H$, Fischer B, Norra C, Schellenberger F, Stender N Stiefel M, Siren AL, Paulus W, Nave KA, Gold R and Bartels C: Exploring recombinant human erythropoietin in chronic progressive multiple sclerosis. Brain 2007, I30:2577-88.

2198. Esiri $\mathrm{MM}$. The interplay between inflammation and neurodegeneration in CNS disease. J Neuroimmunol 2007, I84:4-I6.

2199. Juul S and Felderhoff-Mueser U: Epo and other hematopoietic factors. Semin Fetal Neonatal Med 2007, I2:250-8.

2200. Lykissas MG, Korompilias AV, Vekris MD, Mitsionis GI, Sakellariou $E$ and Beris AE: The role of erythropoietin in central and peripheral nerve injury. Clin Neurol Neurosurg 2007, 109:639-44.

2201. Noguchi CT, Asavaritikrai P, Teng R and Jia Y: Role of erythropoietin in the brain. Crit Rev Oncol Hematol 2007, 64:|59-7|.

2202. Wang Y, Zhang ZG, Rhodes K, Renzi M, Zhang RL, Kapke A, Lu M, Pool C, Heavner G and Chopp M: Post-ischemic treatment with erythropoietin or carbamylated erythropoietin reduces infarction and improves neurological outcome in a rat model of focal cerebral ischemia. Br J Pharmacol 2007, I 5 |: | 377-84.

2203. Wu Y, Shang Y, Sun SG, Liu RG and Yang WQ. Protective effect of erythropoietin against I-methyl-4-phenylpyridiniuminduced neurodegenaration in PCI2 cells. Neurosci Bull 2007, 23: 156-64.

2204. Wiese L, Hempel C, Penkowa M, Kirkby $N$ and Kurtzhals JA: Recombinant human erythropoietin increases survival and reduces neuronal apoptosis in a murine model of cerebral malaria. Malar J 2008, 7:3.

2205. Bianchi R, Buyukakilli B, Brines M, Savino C, Cavaletti G, Oggioni N Lauria G, Borgna M, Lombardi R, Cimen B, Comelekoglu U, Kanik A, Tataroglu C, Cerami A and Ghezzi P: Erythropoietin both protects from and reverses experimental diabetic neuropathy. Proc Natl Acad Sci USA 2004, 1 0 I:823-8.

2206. İnal M, Kanbak G, Șen S, Akyüz F and Sunal E: Antioxidant status and lipid peroxidation in hemodialysis patients undergoing erythropoietin and erythropoietin-vitamin E combined therapy. Free Radic Res 1999, 3 1:21 I-6. 
2207. Yang CW, Li C, Jung JY, Shin SJ, Choi BS, Lim SW, Sun BK, Kim YS, $\mathrm{Kim}$ J, Chang YS and Bang BK: Preconditioning with erythropoietin protects against subsequent ischemia-reperfusion injury in rat kidney. FASEB J 2003, I 7: I 754-5.

2208. Patel NS, Sharples EJ, Cuzzocrea S, Chatterjee PK, Britti D Yaqoob MM and Thiemermann C: Pretreatment with EPO reduces the injury and dysfunction caused by ischemia/ reperfusion in the mouse kidney in vivo. Kidney Int 2004, 66:983-9.

2209. Sharples EJ, Patel N, Brown P, Stewart K, Mota-Philipe H, Sheaff M, Kieswich J, Allen D, Harwood S, Raftery $M$, Thiemermann $C$ and Yaqoob MM: Erythropoietin protects the kidney against the injury and dysfunction caused by ischemia-reperfusion. J Am Soc Nephrol 2004, I 5:21 I5-24.

2210. Ates E, Yalcin AU, Yilmaz S, Koken T and Tokyol C: Protective effect of erythropoietin on renal ischemia and reperfusion injury. ANZ J Surg 2005, 75: I I00-5.

22II. Fliser D, Bahlmann FH and Haller H: EPO: renoprotection beyond anemia correction. Pediatr Nephrol 2006, 21:1785-9.

2212. Jie KE, Verhaar MC, Cramer MJ, Putten van der K, Gaillard CA, Doevendans PA, Koomans HA, Joles JA and Braam B: Erythropoietin and the cardiorenal syndrome: cellular mechanisms on the cardiorenal connectors. Am J Physiol Renal Physiol 2006, 29l:F932-44.

2213. Guneli E, Cavdar Z, Islekel H, Sarioglu S, Erbayraktar S, Kiray M, Sokmen S, Yilmaz $O$ and Gokmen N: Erythropoietin protects the intestine against ischemia/reperfusion injury in rats. Mol Med 2007, 13:509-17.

2214. Mitra A, Bansal S, Wang W, Falk S, Zolty E and Schrier RW: Erythropoietin ameliorates renal dysfunction during endotoxaemia. Nephrol Dial Transplant 2007, 22:2349-53.

2215. Ashikawa K, Majumdar S, Banerjee S, Bharti AC, Shishodia S and Aggarwal BB: Piceatannol inhibits TNF-induced NF-kappaB activation and NF-kappaB-mediated gene expression through suppression of IkappaBalpha kinase and p65 phosphorylation. I Immunol 2002, I 69:6490-7.

2216. Paur I, Austenaa LM and Blomhoff R: Extracts of dietary plants are efficient modulators of nuclear factor kappa B. Food Chem Toxicol 2008, 46: I 288-97.

2217. Wojchowski DM, Gregory RC, Miller CP, Pandit AK and Pircher T]: Signal transduction in the erythropoietin receptor system. Exp Cell Res 1999, 253:143-56.

2218. Coleman T and Brines M: Science review: recombinant human erythropoietin in critical illness: a role beyond anemia? Crit Care 2004, 8:337-4I.

2219. Hanlon PR, Fu P, Wright GL, Steenbergen C, Arcasoy MO and Murphy E: Mechanisms of erythropoietin-mediated cardioprotection during ischemia-reperfusion injury: role of protein kinase $C$ and phosphatidylinositol 3-kinase signaling. FASEB / 2005, 19:1323-5.

2220. Maiese K, Li F and Chong ZZ: Erythropoietin in the brain: can the promise to protect be fulfilled? Trends Pharmacol Sci 2004, 25:577-83.

2221. Chong ZZ, Li F and Maiese K: Erythropoietin requires NFkappaB and its nuclear translocation to prevent early and late apoptotic neuronal injury during beta-amyloid toxicity. Curr Neurovasc Res 2005, 2:387-99.

2222. Maiese K, Li F and Chong ZZ: New avenues of exploration for erythropoietin. JAMA 2005, 293:90-5.

2223. Xu B, Dong GH, Liu H, Wang YQ, Wu HW and Jing $H$ : Recombinant human erythropoietin pretreatment attenuates myocardial infarct size: a possible mechanism involves heat shock Protein 70 and attenuation of nuclear factorkappaB. Ann Clin Lab Sci 2005, 35:16I-8.

2224. Aerbajinai W, Zhu J, Gao Z, Chin K and Rodgers GP: Thalidomide induces gamma-globin gene expression through increased reactive oxygen species-mediated p38 MAPK signaling and histone $\mathrm{H} 4$ acetylation in adult erythropoiesis. Blood 2007, I I 0:2864-7I.

2225. Ryter SW, Kim HP, Hoetzel A, Park JW, Nakahira K, Wang X and Choi AM: Mechanisms of cell death in oxidative stress. Antioxid Redox Signal 2007, 9:49-89.

2226. Wu Y, Shang Y, Sun S, Liang H and Liu R: Erythropoietin prevents PCI 2 cells from I-methyl-4-phenylpyridinium ioninduced apoptosis via the Akt/GSK-3 beta/caspase-3 mediated signaling pathway. Apoptosis 2007, I 2: | 365-75.

2227. Cavdar C, Camsari T, Semin I, Gonenc S and Acikgoz O: Lipid peroxidation and antioxidant activity in chronic haemodialysis patients treated with recombinant human erythropoietin. Scand J Urol Nephrol 1997, 3 I:37I-5.
2228. Katavetin P, Inagi R, Miyata T, Shao J, Sassa R, Adler S, Eto N, Kato $H$, Fujita $T$ and Nangaku M: Erythropoietin induces heme oxygenase-I expression and attenuates oxidative stress. Biochem Biophys Res Commun 2007, 359:928-34.

2229. Katavetin P, Tungsanga K, Eiam-Ong S and Nangaku M: Antioxidative effects of erythropoietin. Kidney Int Suppl 2007, SI0-5.

2230. Chattopadhyay A, Das Choudhury TD, Basu MK and Datta AG: Effect of $\mathrm{Cu}^{2+}$-ascorbic acid on lipid peroxidation, $\mathrm{Mg}^{2+}$. ATPase activity and spectrin of RBC membrane and reversal by erythropoietin. Mol Cell Biochem 1992, I I 8:23-30.

2231. Chattopadhyay A, Choudhury TD, Bandyopadhyay D and Datta AG: Protective effect of erythropoietin on the oxidative damage of erythrocyte membrane by hydroxyl radical. Biochem Pharmacol 2000, 59:419-25.

2232. Akisu M, Tuzun S, Arslanoglu S, Yalaz M and Kultursay N: Effect of recombinant human erythropoietin administration on lipid peroxidation and antioxidant enzyme(s) activities in preterm infants. Acta Med Okayama 200I, 55:357-62.

2233. Hirayama A, Nagase S, Gotoh M, Ueda A, Ishizu T, Yoh K, Aoyagi K, Terao J and Koyama $A$ : Reduced serum hydroxyl radical scavenging activity in erythropoietin therapy resistant renal anemia. Free Radic Res 2002, 36: I I55-6I.

2234. Yankowitz J, Piraino B, Laifer SA, Frassetto L, Gavin L, Kitzmiller JL and Crombleholme W: Erythropoietin in pregnancies complicated by severe anemia of renal failure. Obstet Gynecol 1992, 80:485-8.

2235. Braga J, Marques R, Branco A, Goncalves J, Lobato L, Pimentel JP, Flores MM, Goncalves E and Jorge CS: Maternal and perinatal implications of the use of human recombinant erythropoietin. Acta Obstet Gynecol Scand 1996, 75:449-3.

2236. Jungers $P$ and Chauveau $D$ : Pregnancy in renal disease. Kidney Int 1997, 52:87|-85.

2237. Vora $M$ and Gruslin A: Erythropoietin in obstetrics. Obstet Gynecol Surv 1998, 53:500-8.

2238. Breymann C, Visca E, Huch R and Huch A: Efficacy and safety of intravenously administered iron sucrose with and without adjuvant recombinant human erythropoietin for the treatment of resistant iron-deficiency anemia during pregnancy. Am J Obstet Gynecol 200 I, I 84:662-7.

2239. Sifakis S, Angelakis E, Vardaki E, Koumantaki Y, Matalliotakis I and Koumantakis E: Erythropoietin in the treatment of iron deficiency anemia during pregnancy. Gynecol Obstet Invest 2001, 51:150-6.

2240. Sesti F, Ticconi C, Bonifacio S and Piccione E: Preoperative administration of recombinant human erythropoietin in patients undergoing gynecologic surgery. Gynecol Obstet Invest 2002, 54: I-5.

224I. Donato E, Guinot M, Vilar C, Garcia R and Canigral G: rHuEPO in the management of pregnancy complicated by anti-Dib. Transfusion 2003, 43:681-2.

2242. Kaupke C], Vaziri ND, Powers DR and Gonzales E: Erythropoietin in preeclampsia. Obstet Gynecol 1991, 78:795-9.

2243. Buescher U, Hertwig K, Wolf C and Dudenhausen JW: Erythropoietin in amniotic fluid as a marker of chronic fetal hypoxia. Int J Gynaecol Obstet 1998, 60:257-63.

2244. Goldstein JD, Garry DJ and Maulik D: Obstetric conditions and erythropoietin levels. Am J Obstet Gynecol 2000, I 82:1055-7.

2245. Troeger C, Holzgreve W, Ladewig A, Zhong XY and Hahn S: Examination of maternal plasma erythropoietin and activin A concentrations with regard to circulatory erythroblast levels in normal and preeclamptic pregnancies. Fetal Diagn Ther 2006, 2I:156-60.

2246. Semenza GL: HIF-I and human disease: one highly involved factor. Genes Dev 2000, |4:|983-9|.

2247. Semenza GL: Hypoxia-inducible factor I: oxygen homeostasis and disease pathophysiology. Trends Mol Med 200I, 7:345-50.

2248. Wenger RH: Cellular adaptation to hypoxia: $\mathbf{O}_{2}$-sensing protein hydroxylases, hypoxia-inducible transcription factors, and $\mathbf{O}_{2}$-regulated gene expression. FASEB J 2002 , 16: $1|5|-62$

2249. Pugh CW and Ratcliffe PJ: Regulation of angiogenesis by hypoxia: role of the HIF system. Nat Med 2003, 9:677-84.

2250. Haase VH: Hypoxia-inducible factors in the kidney. Amer J Physiol 2006, 29l:F27I-F28I.

225I. Yoon D, Pastore YD, Divoky V, Liu E, Mlodnicka AE, Rainey K, Ponka P, Semenza GL, Schumacher A and Prchal JT: Hypoxiainducible factor-I deficiency results in dysregulated erythropoiesis signaling and iron homeostasis in mouse development. J Biol Chem 2006, 28I:25703-II. 
2252. Zhang B, Tanaka J, Yang L, Yang L, Sakanaka M, Hata R, Maeda N and Mitsuda N: Protective effect of vitamin $E$ against focal brain ischemia and neuronal death through induction of target genes of hypoxia-inducible factor-I. Neuroscience 2004 , I 26:433-440.

2253. Rius J, Guma M, Schachtrup C, Akassoglou K, Zinkernagel AS, Nizet V, Johnson RS, Haddad GG and Karin M: NF-kappaB links innate immunity to the hypoxic response through transcriptional regulation of HIF-I alpha. Nature 2008.

2254. Peyssonnaux C, Zinkernagel AS, Schuepbach RA, Rankin E, Vaulont S, Haase VH, Nizet $V$ and Johnson RS: Regulation of iron homeostasis by the hypoxia-inducible transcription factors (HIFs). J Clin Invest 2007, I I 7:1926-32.

2255. Peyssonnaux C, Cejudo-Martin P, Doedens A, Zinkernagel AS, Johnson RS and Nizet V: Cutting edge: Essential role of hypoxia inducible factor-Ialpha in development of lipopolysaccharide-induced sepsis. J Immunol 2007, I 78:75 I 6-9.

2256. Peyssonnaux C, Nizet $V$ and Johnson RS: Role of the hypoxia inducible factors HIF in iron metabolism. Cell Cycle 2008, 7:28-32.

2257. Hamrick SE, McQuillen PS, Jiang X, Mu D, Madan A and Ferriero DM: A role for hypoxia-inducible factor-lalpha in desferoxamine neuroprotection. Neurosci Lett 2005, 379:96-100

2258. Caniggia I, Winter J, Lye SJ and Post M: Oxygen and placental development during the first trimester: implications for the pathophysiology of pre-eclampsia. Placenta 2000, 2I (Suppl A):S25-30.

2259. Qutub AA and Popel AS: A computational model of intracellular oxygen sensing by hypoxia-inducible factor HIFI alpha. J Cell Sci 2006, I I 9:3467-80.

2260. Qutub AA and Popel AS: Three autocrine feedback loops determine HIFI alpha expression in chronic hypoxia. Biochim Biophys Acta 2007, I 773: I 5 I I-25.

226I. von Dassow G, Meir E, Munro EM and Odell GM: The segment polarity network is a robust development module. Nature 2000, 406:188-192.

2262. Brandman O, Ferrell JE Jr, Li R and Meyer T: Interlinked fast and slow positive feedback loops drive reliable cell decisions. Science 2005, 3 I 0:496-8.

2263. Kell DB and Knowles JD: The role of modeling in systems biology. System modeling in cellular biology: from concepts to nuts and bolts MIT Press, Cambridge: Szallasi Z, Stelling J, Periwal V 2006, $3-18$.

2264. Wei YH and Lee HC: Oxidative stress, mitochondrial DNA mutation, and impairment of antioxidant enzymes in aging. Exp Biol Med (Maywood) 2002, 227:67I-82.

2265. McCord JM: Iron, free radicals, and oxidative injury. Semin Hematol 1998, 35:5-12.

2266. Barkai $N$ and Leibler S: Robustness in simple biochemical networks. Nature 1997, 387:913-7.

2267. Bornholdt S and Sneppen K: Robustness as an evolutionary principle. Proc. R. Soc. B-Biological Sciences 2000, 267:228I-2286.

2268. Kohn MC, Tohmaz AS, Giroux KJ, Blumenthal GM, Feezor MD and Millington DS: Robustness of MetaNet graph models: Predicting control of urea production in humans. Biosystems 2002, 65:61-78.

2269. Ma $L$ and Iglesias PA: Quantifying robustness of biochemical network models. BMC Bioinformatics 2002, 3: http://www. biomedcentral.com/|47|-2105/3/38.

2270. Morohashi M, Winn AE, Borisuk MT, Bolouri H, Doyle J and Kitano $\mathrm{H}$ : Robustness as a measure of plausibility in models of biochemical networks. J Theor Biol 2002, 2 16: 19-30.

227I. Aldana M and Cluzel P: A natural class of robust networks. Proc Natl Acad Sci USA 2003, 100:8710-4.

2272. Ebenhoh $O$ and Heinrich R: Stoichiometric design of metabolic networks: multifunctionality clusters, optimization, weak and strong robustness. Bull Math Biol 2003, 65:323-57.

2273. Kitano H, Oda K, Kimura T, Matsuoka Y, Csete M, Doyle J and Muramatsu M: Metabolic syndrome and robustness tradeoffs. Diabetes 2004, 53(Suppl 3):S6-SI 5.

2274. Kitano H: Biological robustness. Nat Rev Genet 2004, 5:826-37.

2275. Stelling J, Sauer U, Szallasi Z, Doyle FJ 3rd and Doyle J: Robustness of cellular functions. Cell 2004, I I 8:675-85.

2276. Stelling J, Gilles ED and Doyle FJ 3rd: Robustness properties of circadian clock architectures. Proc Natl Acad Sci USA 2004, I 0 I: : 32 I0-5.

2277. Chen BS, Wang YC, Wu WS and Li WH: A new measure of the robustness of biochemical networks. Bioinformatics 2005, 2 I :2698-705.
2278. Wagner A: Robustness evolvability, and neutrality. FEBS Lett 2005, 579: I772-8.

2279. Wagner A: Circuit topology and the evolution of robustness in two-gene circadian oscillators. Proc Natl Acad Sci USA 2005, 102: 1 1775-80.

2280. Kurata H, El-Samad H, Iwasaki R, Ohtake H, Doyle JC, Grigorova I, Gross CA and Khammash M: Module-based analysis of robustness tradeoffs in the heat shock response system. PLoS Comput Biol 2006, 2:e59.

228I. Wagner A: Robustness and evolvability in living systems Princeton University Press Princeton, NJ; 2005.

2282. Moriya $H$, Shimizu-Yoshida $Y$ and Kitano $H$ : In vivo robustness analysis of cell division cycle genes in Saccharomyces cerevisiae. PLoS Genet 2006, 2: III.

2283. Ma W, Lai L, Ouyang $Q$ and Tang C: Robustness and modular design of the Drosophila segment polarity network. Mol Syst Biol 2006, 2:70.

2284. Kitano $\mathrm{H}$ : A robustness-based approach to systems-oriented drug design. Nat Rev Drug Discov 2007, 6:202-210.

2285. Vizan P, Mazurek S and Cascante M: Robust metabolic adaptation underlying tumor progression. Metabolomics 2008, 4: $1-12$

2286. Jacobsen EW and Cedersund G: Structural robustness of biochemical network models-with application to the oscillatory metabolism of activated neutrophils. IET Syst Biol 2008, 2:39-47.

2287. Tsai TY, Choi YS, Ma W, Pomerening JR, Tang C and Ferrell JE Jr: Robust, tunable biological oscillations from interlinked positive and negative feedback loops. Science 2008, 32 I: $126-9$.

2288. Daniels BC, Chen YJ, Sethna JP, Gutenkunst RN and Myers CR: Sloppiness robustness, and evolvability in systems biology. Curr Opin Biotechnol 2008, 19:389-95.

2289. Martin $O C$ and Wagner $A$ : Multifunctionality and robustness trade-offs in model genetic circuits. Biophys J 2008, 94:2927-37

2290. Wagner A: Robustness and evolvability: a paradox resolved. Proc Biol Sci 2008, 275:91-100.

2291. Lehár J, Krueger A, Zimmermann G and Borisy A: High-order combination effects and biological robustness. Molecular Systems Biology 2008, 4:.

2292. Medawar P: Pluto's republic Oxford University Press, Oxford; 1982

2293. Gilbert GN and Mulkay M: Opening Pandora's box: a sociological analysis of scientists' discourse Cambridge University Press, Cambridge; 1984.

2294. Breen AP and Murphy JA: Reactions of oxyl radicals with DNA. Free Radic Biol Med 1995, I 8: 1033-77.

2295. Burrows CJ and Muller JG: Oxidative nucleobase modifications leading to strand scission. Chem Rev 1998, 98: I I09-I I 52.

2296. Bolton JL, Trush MA, Penning TM, Dryhurst G and Monks TJ: Role of quinones in toxicology. Chem Res Toxicol 2000, I3:|35-60.

2297. Cooke MS, Evans MD, Dizdaroglu $M$ and Lunec J: Oxidative DNA damage: mechanisms mutation, and disease. Faseb J 2003, I7:1195-214.

2298. Haggarty SJ, Clemons PA and Schreiber SL: Chemical genomic profiling of biological networks using graph theory and combinations of small molecule perturbations. I Am Chem Soc 2003, I 25: 10543-5.

2299. Csermely P, Agoston $V$ and Pongor S: The efficiency of multitarget drugs: the network approach might help drug design. Trends Pharmacol Sci 2005, 26: I78-82.

2300. Ambesi-Impiombato A and di Bernardo D: Computational biology and drug discovery: From single-target to network drugs. Current Bioinformatics 2006, I:3-13.

230I. Yeh P, Tschumi Al and Kishony R: Functional classification of drugs by properties of their pairwise interactions. Nat Genet 2006, 38:489-94.

2302. Lee MS, Johansen L, Zhang Y, Wilson A, Keegan M, Avery W, Elliott $P$, Borisy AA and Keith CT: The novel combination of chlorpromazine and pentamidine exerts synergistic antiproliferative effects through dual mitotic action. Cancer Res 2007, 67: II359-67.

2303. Abbott A: Pharmaceutical futures: a fiendish puzzle. Nature 2008, 455: I |64-7.

2304. Henney A and Superti-Furga G: A network solution. Nature 2008, 455:730-I.

2305. Hoon S, Smith AM, Wallace IM, Suresh S, Miranda M, Fung E, Proctor M, Shokat KM, Zhang C, Davis RW, Giaever G, St Onge RP and Nislow C: An integrated platform of genomic assays 
reveals small-molecule bioactivities. Nat Chem Biol 2008, 4:498-506.

2306. Kvien TK, Fjeld E, Slatkowsky-Christensen B, Nichols M, Zhang Y, Proven A, Mikkelsen K, Palm O, Borisy AA and Lessem J: Efficacy and safety of a novel synergistic drug candidate, CRx-I02, in hand osteoarthritis. Ann Rheum Dis 2008, 67:942-8.

2307. Lehár J, Stockwell BR, Giaever G and Nislow C: Combination chemical genetics. Nat Chem Biol 2008, 4:674-8I.

2308. Nelander S, Wang W, Nilsson B, She QB, Pratilas C, Rosen N, Gennemark P and Sander C: Models from experiments: combinatorial drug perturbations of cancer cells. Mol Syst Biol 2008, 4:216.

2309. Westerhoff HV: Systems biology: the grand challenge for European biology. Screening 2008, 9:34-37.

2310. Westerhoff HV, Mosekilde E, Noe CR and Clemensen AM: Integrating systems approaches into pharmaceutical sciences. Eur J Pharm Sci 2008, 35: $1-4$.

23II. Tong AH, Lesage G, Bader GD, Ding $H, X u H, X i n ~ X$, Young J, Berriz GF, Brost RL, Chang $M$, Chen $Y$, Cheng $X$, Chua G, Friesen H, Goldberg DS, Haynes J, Humphries C, He G, Hussein S, Ke L, Krogan N, Li Z, Levinson JN, Lu H, Menard P, Munyana C, Parsons AB, Ryan O, Tonikian R, Roberts T, Sdicu AM, Shapiro J, Sheikh B, Suter B, Wong SL, Zhang LV, Zhu H, Burd CG, Munro S, Sander C, Rine J, Greenblatt J, Peter M, Bretscher A, Bell G, Roth FP, Brown GW, Andrews B, Bussey $\mathrm{H}$ and Boone C: Global mapping of the yeast genetic interaction network. Science 2004, 303:808-13.

23I2. Parsons AB, Brost RL, Ding H, Li Z, Zhang C, Sheikh B, Brown GW, Kane PM, Hughes TR and Boone C: Integration of chemicalgenetic and genetic interaction data links bioactive compounds to cellular target pathways. Nat Biotechnol 2004, 22:62-9.

2313. Parsons AB, Lopez A, Givoni IE, Williams DE, Gray CA, Porter J, Chua G, Sopko R, Brost RL, Ho CH, Wang J, Ketela T, Brenner C, Brill JA, Fernandez GE, Lorenz TC, Payne GS, Ishihara S, Ohya Y, Andrews B, Hughes TR, Frey BJ, Graham TR, Andersen RJ and Boone $C$ : Exploring the mode-of-action of bioactive compounds by chemical-genetic profiling in yeast. Cell 2006, 126:6 II-25.

2314. Pearl J: Probabilistic reasoning in intelligent systems: networks of plausible inference Morgan Kaufmann, San Francisco; 1988.

23I5. Pearl J: Causality: models, reasoning and inference Cambridge University Press, Cambridge; 2000.

2316. Hastie T, Tibshirani R and Friedman J: The elements of statistical learning: data mining, inference and prediction Springer-Verlag, Berlin; 2001.

2317. Shipley B: Cause and correlation in biology: a user's guide to path analysis, structural equations and causal inference Cambridge University Press, Cambridge; $200 \mathrm{I}$.

2318. Mackay DJC: Information theory, inference and learning algorithms Cambridge University Press, Cambridge; 2003.

2319. Needham CJ, Bradford JR, Bulpitt AJ and Westhead DR: Inference in Bayesian networks. Nat Biotechnol 2006, 24:5I-53.

2320. Jayawardhana $B$, Kell DB and Rattray $M$ : Bayesian inference of the sites of perturbations in metabolic pathways via Markov Chain Monte Carlo. Bioinformatics 2008, 24: I I91-I I97.

232I. Rohr JR, Raffel TR, Sessions SK and Hudson PJ: Understanding the net effects of pesticides on amphibian trematode infections. Ecol Appl 2008, I8: 1743-53.

2322. Calabrese EJ: Converging concepts: adaptive response preconditioning, and the Yerkes-Dodson Law are manifestations of hormesis. Ageing Res Rev 2008, 7:8-20.

2323. Calabrese EJ: Hormesis and medicine. Br J Clin Pharmacol 2008.

2324. Calabrese E: Hormesis: why it is important to toxicology and toxicologists. Environ Toxicol Chem 2008, I.

2325. Mattson MP: Hormesis and disease resistance: activation of cellular stress response pathways. Hum Exp Toxicol 2008, 27:155-62.

2326. Mattson MP: Hormesis defined. Ageing Res Rev 2008, 7: I-7.

2327. The Long-Term Intervention with Pravastatin in Ischaemic Disease (LIPID) Study Group: Prevention of cardiovascular events and death with pravastatin in patients with coronary heart disease and a broad range of initial cholesterol levels. $N$ Engl J Med 1998, 339:1349-57.

2328. Liappis AP, Kan VL, Rochester CG and Simon GL: The effect of statins on mortality in patients with bacteremia. Clin Infect Dis 2001, 33:1352-7.

2329. Almog Y, Shefer A, Novack V, Maimon N, Barski L, Eizinger M, Friger M, Zeller L and Danon A: Prior statin therapy is associated with a decreased rate of severe sepsis. Circulation 2004, I I 0:880-5.

2330. Merx MW, Liehn EA, Janssens U, Lutticken R, Schrader J, Hanrath $P$ and Weber C: HMG-CoA reductase inhibitor simvastatin profoundly improves survival in a murine model of sepsis. Circulation 2004, 109:2560-5.

2331. Merx MW, Liehn EA, Graf J, Sandt van de A, Schaltenbrand M, Schrader J, Hanrath $P$ and Weber C: Statin treatment after onset of sepsis in a murine model improves survival. Circulation 2005, I I 2: I I7-24.

2332. Zhang L, Zhang ZG, Ding GL, Jiang Q, Liu X, Meng $H$, Hozeska A, Zhang C, Li L, Morris D, Zhang RL, Lu M and Chopp M: Multitargeted effects of statin-enhanced thrombolytic therapy for stroke with recombinant human tissue-type plasminogen activator in the rat. Circulation 2005, I I 2:3486-94.

2333. Hackam DG, Mamdani M, Li P and Redelmeier DA: Statins and sepsis in patients with cardiovascular disease: a populationbased cohort analysis. Lancet 2006, 367:4I3-8.

2334. Terblanche M, Almog Y, Rosenson RS, Smith TS and Hackam DG: Statins: panacea for sepsis? Lancet Infect Dis 2006, 6:242-8.

2335. Thomsen RW, Hundborg HH, Johnsen SP, Pedersen L, Sorensen HT, Schonheyder HC and Lervang $\mathrm{HH}$ : Statin use and mortality within 180 days after bacteremia: a populationbased cohort study. Crit Care Med 2006, 34:1080-6.

2336. Aneja R and Fink MP: Promising therapeutic agents for sepsis. Trends Microbiol 2007, I 5:3 I-7.

2337. Martin CP, Talbert RL, Burgess DS and Peters Jl: Effectiveness of statins in reducing the rate of severe sepsis: a retrospective evaluation. Pharmacotherapy 2007, 27:20-6.

2338. Merx MW and Weber C: Sepsis and the heart. Circulation 2007, I 1 6:793-802.

2339. Schlienger RG, Fedson DS, Jick SS, Jick $H$ and Meier CR: Statins and the risk of pneumonia: a population-based, nested case-control study. Pharmacotherapy 2007, 27:325-32.

2340. Falagas ME, Makris GC, Matthaiou DK and Rafailidis PI: Statins for infection and sepsis: a systematic review of the clinical evidence. J Antimicrob Chemother 2008, 61:774-85.

234 I. Lipinski MJ, Abbate A, Fuster V and Vetrovec GW: Drug insight: statins for nonischemic heart failure-evidence and potential mechanisms. Nat Clin Pract Cardiovasc Med 2007, 4: I 96-205.

2342. Santodomingo-Garzón T, Cunha TM, Verri WA Jr, Valerio DA, Parada CA, Poole S, Ferreira SH and Cunha FQ: Atorvastatin inhibits inflammatory hypernociception. Br J Pharmacol 2006, |49: |4-22.

2343. Ferreira GA, Navarro TP, Telles RW, Andrade LE and Sato El: Atorvastatin therapy improves endothelial-dependent vasodilation in patients with systemic lupus erythematosus: an 8 weeks controlled trial. Rheumatology (Oxford) 2007, 46: $1560-5$.

2344. Rydgren T, Vaarala $O$ and Sandler S: Simvastatin protects against multiple low-dose streptozotocin-induced type I diabetes in CD-I mice and recurrence of disease in nonobese diabetic mice. I Pharmacol Exp Ther 2007, 323:|80-5.

2345. Leung BP, Sattar N, Crilly A, Prach M, McCarey DW, Payne H, Madhok R, Campbell C, Gracie JA, Liew FY and Mclnnes IB: A novel anti-inflammatory role for simvastatin in inflammatory arthritis. J Immunol 2003, I 70: I 524-30.

2346. Sattar N, McCarey DW, Capell H and Mclnnes IB: Explaining how "high-grade" systemic inflammation accelerates vascular risk in rheumatoid arthritis. Circulation 2003, 108:2957-63.

2347. Barsante MM, Roffe E, Yokoro CM, Tafuri WL, Souza DG, Pinho V, Castro MS and Teixeira MM: Anti-inflammatory and analgesic effects of atorvastatin in a rat model of adjuvant-induced arthritis. Eur J Pharmacol 2005, 5 1 6:282-9.

2348. Dombrecht EJ, Van Offel JF, Bridts CH, Ebo DG, Seynhaeve V, Schuerwegh AJ, Stevens WJ and De Clerck LS: Influence of simvastatin on the production of pro-inflammatory cytokines and nitric oxide by activated human chondrocytes. Clin Exp Rheumatol 2007, 25:534-9.

2349. Haruna Y Morita Y, Yada T, Satoh M, Fox DA and Kashihara N Fluvastatin reverses endothelial dysfunction and increased vascular oxidative stress in rat adjuvant-induced arthritis. Arthritis Rheum 2007, 56:1827-35.

2350. Yamagata T, Kinoshita K, Nozaki Y, Sugiyama M, Ikoma S and Funauchi $M$ : Effects of pravastatin in murine collageninduced arthritis. Rheumatol Int 2007, 27:631-9.

235I. Epstein M and Campese VM: Pleiotropic effects of 3-hydroxy-3methylglutaryl coenzyme a reductase inhibitors on renal function. Am J Kidney Dis 2005, 45:2-I4. 
2352. Yasuda H, Yuen PS, Hu X, Zhou H and Star RA: Simvastatin improves sepsis-induced mortality and acute kidney injury via renal vascular effects. Kidney Int 2006, 69:1535-42.

2353. Campese VM and Park J: HMG-CoA reductase inhibitors and the kidney. Kidney Int 2007, 7I:I2I5-22.

2354. Otuki MF, Pietrovski EF and Cabrini DA: Topical simvastatin: preclinical evidence for a treatment of skin inflammatory conditions. J Dermatol Sci 2006, 44:45-7.

2355. Lee JH, Lee DS, Kim EK, Choe KH, Oh YM, Shim TS, Kim SE, Lee YS and Lee SD: Simvastatin inhibits cigarette smokinginduced emphysema and pulmonary hypertension in rat lungs. Am J Respir Crit Care Med 2005, I 72:987-93.

2356. Pirat A, Zeyneloglu P, Aldemir D, Yücel M, Özen O, Candan S and Arslan $G$ : Pretreatment with simvastatin reduces lung injury related to intestinal ischemia-reperfusion in rats. Anesth Analg 2006, 102:225-32.

2357. Endres M, Laufs U, Huang Z, Nakamura T, Huang P, Moskowitz MA and Liao JK: Stroke protection by 3-hydroxy-3-methylglutaryl (HMG)-CoA reductase inhibitors mediated by endothelial nitric oxide synthase. Proc Natl Acad Sci USA 1998, 95:8880-5

2358. Vaughan CJ and Delanty N: Neuroprotective properties of statins in cerebral ischemia and stroke. Stroke 1999 , 30:1969-73.

2359. Amin-Hanjani S, Stagliano NE, Yamada M, Huang PL, Liao JK and Moskowitz MA: Mevastatin, an HMG-CoA reductase inhibitor, reduces stroke damage and upregulates endothelial nitric oxide synthase in mice. Stroke 2001, 32:980-6.

2360. Sironi L, Cimino M, Guerrini U, Calvio AM, Lodetti B, Asdente M, Balduini $W$, Paoletti $R$ and Tremoli $E$ : Treatment with statins after induction of focal ischemia in rats reduces the extent of brain damage. Arterioscler Thromb Vasc Biol 2003, 23:322-7.

236I. Endres M, Laufs U, Liao JK and Moskowitz MA: Targeting eNOS for stroke protection. Trends Neurosci 2004, 27:283-9.

2362. Amarenco $P$ and Moskowitz MA: The dynamics of statins: from event prevention to neuroprotection. Stroke 2006, 37:294-6.

2363. Amarenco P, Lavallee PC, Mazighi M and Labreuche J: The role of statins in the prevention of stroke. Archives of Medical Science 2007, 3:SI09-SII4.

2364. DeFaria Yeh D and Waters DD: Preventing and treating stroke and transient ischemic attack. Am J Cardiol 2008, I0I:270-3.

2365. Chen SF, Hung TH, Chen CC, Lin KH, Huang YN, Tsai HC and Wang JY: Lovastatin improves histological and functional outcomes and reduces inflammation after experimental traumatic brain injury. Life Sci 2007, 81:288-98.

2366. Faden $\mathrm{Al}$ and Stoica $\mathrm{B}$ : Neuroprotection: challenges and opportunities. Arch Neurol 2007, 64:794-800.

2367. Wu H, Lu D, Jiang H, Xiong Y, Qu C, Li B, Mahmood A, Zhou D and Chopp M: Simvastatin-mediated upregulation of VEGF and BDNF, activation of the PI3K/AKt pathway, and increase of neurogenesis are associated with therapeutic improvement after traumatic brain injury. I Neurotrauma 2008, 25:130-9.

2368. Jick H, Zornberg GL, Jick SS, Seshadri S and Drachman DA: Statins and the risk of dementia. Lancet 2000, 356:|627-31.

2369. Wolozin B, Kellman W, Ruosseau P, Celesia GG and Siegel G: Decreased prevalence of Alzheimer disease associated with 3-hydroxy-3-methyglutaryl coenzyme A reductase inhibitors. Arch Neurol 2000, 57:1439-43.

2370. Fassbender K, Simons M, Bergmann C, Stroick M, Lutjohann D, Keller P, Runz H, Kuhl S, Bertsch T, von Bergmann K, Hennerici M, Beyreuther K and Hartmann T: Simvastatin strongly reduces levels of Alzheimer's disease beta -amyloid peptides Abeta 42 and Abeta 40 in vitro and in vivo. Proc Natl Acad Sci USA 2001, 98:5856-6I.

237I. Refolo LM, Pappolla MA, LaFrancois J, Malester B, Schmidt SD, Thomas-Bryant T, Tint GS, Wang R, Mercken M, Petanceska SS and Duff KE: A cholesterol-lowering drug reduces beta-amyloid pathology in a transgenic mouse model of Alzheimer's disease. Neurobiol Dis 2001, 8:890-9.

2372. Hajiar I, Schumpert J, Hirth V, Wieland D and Eleazer GP: The impact of the use of statins on the prevalence of dementia and the progression of cognitive impairment. J Gerontol A Biol Sci Med Sci 2002, 57:M414-8.

2373. Zamvil SS and Steinman L: Cholesterol-lowering statins possess anti-inflammatory activity that might be useful for treatment of MS. Neurology 2002, 59:970-I.

2374. Aktas O, Waiczies S, Smorodchenko A Dorr J Seeger B Prozorovski T, Sallach S, Endres M, Brocke S, Nitsch R and Zipp F: Treatment of relapsing paralysis in experimental encephalomyelitis by targeting ThI cells through atorvastatin. I Exp Med 2003, 197:725-33.

2375. Casserly I and Topol E: Convergence of atherosclerosis and Alzheimer's disease: inflammation cholesterol, and misfolded proteins. Lancet 2004, 363: I |39-46.

2376. Nath N, Giri S, Prasad R, Singh AK and Singh I: Potential targets of 3-hydroxy-3-methylglutaryl coenzyme A reductase inhibitor for multiple sclerosis therapy. I Immunol 2004, I 72: $1273-86$

2377. Neuhaus O, Stuve O, Zamvil SS and Hartung HP: Are statins a treatment option for multiple sclerosis? Lancet Neurol 2004, 3:369-7I.

2378. Vollmer T, Key L, Durkalski V, Tyor W, Corboy J, Markovic-Plese S, Preiningerova ], Rizzo $M$ and Singh I: Oral simvastatin treatment in relapsing-remitting multiple sclerosis. Lancet 2004, 363: $1607-8$

2379. Neuhaus O, Stuve O, Zamvil SS and Hartung HP: Evaluation of HMG-CoA reductase inhibitors for multiple sclerosis: opportunities and obstacles. CNS Drugs 2005, | 9:833-4|.

2380. Mok SW, Thelen KM, Riemer C, Bamme T, Gultner S, Lutjohann D and Baier $M$ : Simvastatin prolongs survival times in prion infections of the central nervous system. Biochem Biophys Res Commun 2006, 348:697-702.

238I. Schmeer C, Kretz A and Isenmann S: Statin-mediated protective effects in the central nervous system: general mechanisms and putative role of stress proteins. Restor Neurol Neurosci 2006, 24:79-95.

2382. Wolozin B, Wang SW, Li NC, Lee A, Lee TA and Kazis LE Simvastatin is associated with a reduced incidence of dementia and Parkinson's disease. BMC Med 2007, 5:20.

2383. Cavalli A Bolognesi ML, Minarini A Rosini M, Tumiatti V, Recanatini $M$ and Melchiorre $C$ : Multi-target-directed ligands to combat neurodegenerative diseases. J Med Chem 2008, 5 I:347-72.

2384. Paul F, Waiczies S, Wuerfel J, Bellmann-Strobl J, Dorr J, Waiczies H, Haertle M, Wernecke KD, Volk HD, Aktas O and Zipp F: Oral high-dose atorvastatin treatment in relapsing-remitting multiple sclerosis. PLOS ONE 2008, 3:el 928.

2385. Bösel J, Gandor F, Harms C, Synowitz M, Harms U, Djoufack PC, Megow D, Dirnagl U, Hortnagl H, Fink KB and Endres $M$ : Neuroprotective effects of atorvastatin against glutamate-induced excitotoxicity in primary cortical neurones. J Neurochem 2005, 92:1386-98.

2386. Wong WW, Dimitroulakos J, Minden MD and Penn LZ: HMGCoA reductase inhibitors and the malignant cell: the statin family of drugs as triggers of tumor-specific apoptosis. Leukemia 2002, 16:508-19.

2387. Kaushal V, Kohli M, Mehta P and Mehta JL: Potential anticancer effects of statins: fact or fiction? Endothelium 2003, 10:49-58.

2388. Wu J, Wong WW, Khosravi F, Minden MD and Penn LZ: Blocking the Raf/MEK/ERK pathway sensitizes acute myelogenous leukemia cells to lovastatin-induced apoptosis. Cancer Res 2004, 64:646I-8.

2389. Demierre MF, Higgins PD, Gruber SB, Hawk E and Lippman SM Statins and cancer prevention. Nat Rev Cancer 2005, 5:930-42.

2390. Fritz G: HMG-CoA reductase inhibitors (statins) as anticancer drugs (review). Int I Oncol 2005, 27:|40|-9.

239l. Katz MS: Therapy insight: Potential of statins for cancer chemoprevention and therapy. Nat Clin Pract Oncol 2005, 2:82-9.

2392. Shannon J, Tewoderos S, Garzotto M, Beer TM, Derenick $R$ Palma $A$ and Farris PE: Statins and prostate cancer risk: a case-control study. Am J Epidemiol 2005, I 62:3 I8-25.

2393. Campbell MJ. Esserman LJ Zhou Y Shoemaker M, Lobo M, Borman E, Baehner F, Kumar AS, Adduci K, Marx C, Petricoin EF, Liotta LA, Winters M, Benz S and Benz CC: Breast cancer growth prevention by statins. Cancer Res 2006, 66:8707-14.

2394. Cauley JA, McTiernan A, Rodabough RJ, LaCroix A, Bauer DC, Margolis KL, Paskett ED, Vitolins MZ, Furberg $C D$ and Chlebowski RT: Statin use and breast cancer: prospective results from the Women's Health Initiative. J Natl Cancer Inst 2006, 98:700-7.

2395. Platz EA, Leitzmann MF, Visvanathan K, Rimm EB, Stampfer MJ, Willett WC and Giovannucci E. Statin drugs and risk of advanced prostate cancer. J Natl Cancer Inst 2006, 98: I8I9-25.

2396. Jacobs EJ, Rodriguez C, Bain EB, Wang Y, Thun MJ and Calle EE: Cholesterol-lowering drugs and advanced prostate cancer incidence in a large U.S. cohort. Cancer Epidemiol Biomarkers Prev 2007, 16:2213-7. 
2397. Kodach LL, Bleuming SA, Peppelenbosch MP, Hommes DW, Brink van den GR and Hardwick JC: The effect of statins in colorectal cancer is mediated through the bone morphogenetic protein pathway. Gastroenterology 2007, I33:|272-8I.

2398. Farwell WR, Scranton RE, Lawler EV, Lew RA, Brophy MT, Fiore LD and Gaziano JM: The association between statins and cancer incidence in a veterans population. J Natl Cancer Inst 2008, 100:134-9.

2399. Karp I, Behlouli $\mathrm{H}$, Lelorier J and Pilote L: Statins and cancer risk. Am J Med 2008, I 2 I:302-9.

2400. Sassano A and Platanias LC: Statins in tumor suppression. Cancer Lett 2008, 260: II-9.

240I. Brenner S: Loose ends Current Biology, London; 1997

2402. Kacser $\mathrm{H}$ : On parts and wholes in metabolism. The organization of cell metabolism Plenum Press, New York: Welch GR, Clegg JS 1986, 327-337.

2403. Pennisi E: A new window on how genomes work. Science 2007, 316:II20-1.

2404. Kell DB and Oliver SG: Here is the evidence, now what is the hypothesis? The complementary roles of inductive and hypothesis-driven science in the post-genomic era. Bioessays 2004, 26:99-105.

2405. Russell B: Am I an atheist or an agnostic? I947.

2406. Kostoff RN: Overcoming specialization. Bioscience 2002, 52:937-94l.

2407. Noble D: Modeling the heart - from genes to cells to the whole organ. Science 2002, 295:1678-1682.

2408. Hunter PJ: The IUPS Physiome Project: a framework for computational physiology. Prog Biophys Mol Biol 2004, 85:551-69.

2409. Duarte NC, Becker SA, Jamshidi N, Thiele I, Mo ML, Vo TD, Srvivas $\mathrm{R}$ and Palsson $\mathrm{B} \varnothing$ : Global reconstruction of the human metabolic network based on genomic and bibliomic data. Proc Natl Acad Sci 2007, I04: I777-1782.

2410. Kell DB: The virtual human: towards a global systems biology of multiscale, distributed biochemical network models. IUBMB Life 2007, 59:689-95.

24II. Ma H, Sorokin A, Mazein A, Selkov A, Selkov E, Demin O and Goryanin I: The Edinburgh human metabolic network reconstruction and its functional analysis. Mol Syst Biol 2007, 3: 135 .

24I2. Feist $A M$ and Palsson $B \varnothing$ : The growing scope of applications of genome-scale metabolic reconstructions using Escherichia coli. Nat Biotechnol 2008, 26(6):659-67.

24I3. Ma H and Goryanin I: Human metabolic network reconstruction and its impact on drug discovery and development. Drug Discov Today 2008, 13:402-8.

24I4. Jamshidi $N$ and Palsson $B \varnothing$ : Formulating genome-scale kinetic models in the post-genome era. Mol Syst Biol 2008, 4: I7I.

24I5. Clapworthy G, Viceconti M, Coveney PV and Kohl P: The virtual physiological human: building a framework for computational biomedicine I. Editorial. Philos Transact A Math Phys Eng Sci 2008, 366:2975-8.

2416. Fenner JW, Brook B, Clapworthy G, Coveney PV, Feipel V Gregersen H, Hose DR, Kohl P, Lawford P, McCormack KM, Pinney D, Thomas SR, Van Sint Jan S, Waters S and Viceconti M: The EuroPhysiome, STEP and a roadmap for the virtual physiological human. Philos Transact A Math Phys Eng Sci 2008, 366:2979-99.

24I7. Snoep JL, Bruggeman F, Olivier BG and Westerhoff HV: Towards building the silicon cell: a modular approach. Biosystems 2006 83:207-16

24I8. Jongeneel CV, Delorenzi M, Iseli C, Zhou D, Haudenschild CD, Khrebtukova I, Kuznetsov D, Stevenson BJ, Strausberg RL, Simpson AJ and Vasicek TJ: An atlas of human gene expression from massively parallel signature sequencing (MPSS). Genome Res 2005, 15:1007-14.

2419. Persson A, Hober S and Uhlen M: A human protein atlas based on antibody proteomics. Curr Opin Mol Ther 2006, 8: I85-90.

2420. Lein ES, Hawrylycz MJ, Ao N, Ayres M, Bensinger A, Bernard A, Boe AF, Boguski MS, Brockway KS, Byrnes EJ, Chen L, Chen L, Chen TM, Chin MC, Chong J, Crook BE, Czaplinska A, Dang CN, Datta S, Dee NR, Desaki AL, Desta T, Diep E, Dolbeare TA, Donelan MJ, Dong HW, Dougherty JG, Duncan BJ, Ebbert AJ Eichele G, Estin LK, Faber C, Facer BA, Fields R, Fischer SR, Fliss TP, Frensley C, Gates SN, Glattfelder KJ, Halverson KR, Hart MR, Hohmann JG, Howell MP, Jeung DP, Johnson RA, Karr PT, Kawal R, Kidney JM, Knapik RH, Kuan CL, Lake JH, Laramee AR, Larsen KD, Lau C, Lemon TA, Liang AJ, Liu Y, Luong LT, Michaels ] Morgan JJ, Morgan RJ, Mortrud MT, Mosqueda NF, Ng LL, Ng R,
Orta GJ, Overly CC, Pak TH, Parry SE, Pathak SD, Pearson OC, Puchalski RB, Riley ZL, Rockett HR, Rowland SA, Royall J], Ruiz MJ, Sarno NR, Schaffnit K, Shapovalova NV, Sivisay T, Slaughterbeck CR, Smith SC, Smith KA, Smith BI, Sodt AJ, Stewart NN, Stumpf KR, Sunkin SM, Sutram M, Tam A Teemer CD, Thaller C, Thompson CL, Varnam LR, Visel A, Whitlock RM, Wohnoutka PE, Wolkey CK and Wong VY, et al: Genome-wide atlas of gene expression in the adult mouse brain. Nature 2007, 445:168-76.

242I. Hober S and Uhlén M: Human protein atlas and the use of microarray technologies. Curr Opin Biotechnol 2008, 19:30-5.

2422. Barbe L, Lundberg E, Oksvold $P$, Stenius A, Lewin E, Björling $E$ Asplund A, Ponten F, Brismar $\mathrm{H}$, Uhlén $M$ and Andersson-Svahn $\mathrm{H}$ : Toward a confocal subcellular atlas of the human proteome. Mol Cell Proteomics 2008, 7:499-508.

2423. Sultan $M$, Schulz MH, Richard $H$, Magen A, Klingenhoff $A$, Scherf $M$, Seifert M, Borodina T, Soldatov A, Parkhomchuk D, Schmidt D, O'Keeffe S, Haas S, Vingron M, Lehrach $\mathrm{H}$ and Yaspo ML: A global view of gene activity and alternative splicing by deep sequencing of the human transcriptome. Science 2008 , $321: 956-60$

2424. Shlomi T, Cabili MN, Herrgard MJ, Palsson B $\varnothing$ and Ruppin E: Network-based prediction of human tissue-specific metabolism. Nat Biotechnol 2008, 26:1003-10.

2425. Hucka M, Finney A, Sauro HM, Bolouri H, Doyle JC, Kitano $H$, Arkin AP, Bornstein BJ, Bray D, Cornish-Bowden A, Cuellar AA, Dronov S, Gilles ED, Ginkel M, Gor V, Goryanin II, Hedley W], Hodgman TC, Hofmeyr JH, Hunter PJ, Juty NS, Kasberger JL, Kremling A, Kummer U, Le Novere N, Loew LM, Lucio D, Mendes P, Minch E, Mjolsness ED, Nakayama Y, Nelson MR, Nielsen PF, Sakurada T, Schaff JC, Shapiro BE, Shimizu TS, Spence HD, Stelling J, Takahashi K, Tomita M, Wagner J and Wang J: The systems biology markup language (SBML): a medium for representation and exchange of biochemical network models. Bioinformatics 2003, 19:524-3I.

2426. Le Novère N, Finney A, Hucka M, Bhalla US, Campagne F, ColladoVides J, Crampin EJ, Halstead M, Klipp E, Mendes P, Nielsen P, Sauro H, Shapiro B, Snoep JL, Spence HD and Wanner BL: Minimum information requested in the annotation of biochemical models (MIRIAM). Nat Biotechnol 2005, 23:1509-I5.

2427. Taylor CF, Field D, Sansone SA, Aerts Apweiler R, Ashburner M Ball CA, Binz PA, Bogue M, Booth T, Brazma A, Brinkman RR, Michael Clark A, Deutsch EW, Fiehn O, Fostel J, Ghazal P, Gibson F, Gray T, Grimes G, Hancock JM, Hardy NW, Hermjakob $\mathrm{H}$, Julian RK Jr, Kane $M$, Kettner C, Kinsinger C, Kolker E, Kuiper M, Novere NL, Leebens-Mack J, Lewis SE, Lord P, Mallon AM, Marthandan N, Masuya H, McNally R, Mehrle A, Morrison N, Orchard S, Quackenbush J, Reecy JM, Robertson DG, Rocca-Serra P, Rodriguez H, Rosenfelder H, Santoyo-Lopez J, Scheuermann RH, Schober D, Smith B, Snape J, Stoeckert C], Tipton K Jr, Sterk P, Untergasser A, Vandesompele J and Wiemann S: Promoting coherent minimum reporting guidelines for biological and biomedical investigations: the MIBBI project. Nat Biotechnol 2008, 26:889-96.

2428. Kell DB: Progress being made on standards for use in data sharing. Nature 2008, 456:29.

2429. Kell DB and Mendes P: The markup is the model: reasoning about systems biology models in the Semantic Web era. Theoret Biol 2008, 252:538-543.

2430. Surowiecki J: The wisdom of crowds: why the many are smarter than the few Abacus, London; 2004.

2431. Anderson CM: The long tail: how endless choice is creating unlimited demand Random House, London; 2006.

2432. Sunstein CR: Infotopia: how many minds produce knowledge Oxfor University Press, Oxford; 2006

2433. Tapscott D and Williams A: Wikinomics: how masscollaboration changes everything New Paradigm; 2007.

2434. Leadbeater C: We-think Profile Books, London; 2008.

2435. Shirky C: Here comes everybody Allen Lane, London; 2008.

2436. Li P, Oinn T, Soiland S and Kell DB: Automated manipulation of systems biology models using libSBML within Taverna workflows. Bioinformatics 2008, 24:287-289.

2437. Li P, Castrillo II, Velarde G, Wassink I, Soiland-Reyes S, Owen S Withers D, Oinn T, Pocock MR, Goble CA, Oliver SG and Kell DB: Performing statistical analyses on quantitative data in Taverna workflows: an example using $\mathbf{R}$ and maxdBrowse to identify differentially expressed genes from microarray data. BMC Bioinformatics 2008, 9:334. 
2438. De Roure D and Goble C: ${ }^{\text {my}}$ Experiment - A Web 2.0 Virtual Research Environment. Proc International Workshop on Virtual Research Environments and Collaborative Work Environments, May 2007, Edinburgh, UK 2007 http://eprints.ecs.soton.ac.uk//396 I/.

2439. Suber P: Open access to the scientific journal literature. J Biol 2002, I:3.

2440. Bourne PE, Fink JL and Gerstein M: Open access: taking full advantage of the content. PLoS Comput Biol 2008, 4:el000037.

244I. Johnson RK: Institutional Repositories: partnering with faculty to enhance scholarly communication. D-Lib Magazine 2002, 8: http://www.dlib.org/dlib/november02/johnson/l ljohnson. html.

2442. Lynch CA: Institutional repositories: Essential infrastructure for scholarship in the digital age. Libr Acad 2003, 3:327-336.

2443. Lee E, Chuang HY, Kim JW, Ideker T and Lee D: Inferring pathway activity toward precise disease classification. PLOS Comput Biol 2008, 4:el0002I7.

2444. Stein LD: Integrating biological databases. Nat Rev Genet 2003, 4:337-45.

2445. Lord P, Bechhofer S, Wilkinson MD, Schiltz G, Gessler D, Hull D, Goble CA and Stein L: Applying Semantic Web services to bioinformatics: Experiences gained, lessons learnt. LNCS 2004, 3298:350-364.

2446. Curcin V, Ghanem $M$ and Guo $Y$ : Web services in the life sciences. Drug Discov Today 2005, 10:865-7I.

2447. Neerincx PB and Leunissen JA: Evolution of web services in bioinformatics. Brief Bioinform 2005, 6:178-88.

2448. Le Novère $N$, Bornstein $B$, Broicher $A$, Courtot $M$, Donizelli $M$, Dharuri H, Li L, Sauro H, Schilstra M, Shapiro B, Snoep JL and Hucka M: BioModels Database: a free, centralized database of curated published, quantitative kinetic models of biochemical and cellular systems. Nucleic Acids Res 2006, 34: D689-9I.

2449. Richardson L and Riuby S: RESTful web services O'Reilly Sebastopol, CA; 2007.

2450. Fernández JM, Hoffmann $R$ and Valencia A: iHOP web services. Nucleic Acids Res 2007, 35:W21-6.

245I. Stein LD: Towards a cyberinfrastructure for the biological sciences: progress, visions and challenges. Nat Rev Genet 2008, 9:678-88.

2452. Williams AJ: A perspective of publicly accessible/open-access chemistry databases. Drug Discov Today 2008, I3:495-50 I.

2453. Margulies M, Egholm M, Altman WE, Attiya S, Bader JS, Bemben LA, Berka J, Braverman MS, Chen YJ, Chen Z, Dewell SB, Du L, Fierro JM, Gomes XV, Godwin BC, He W, Helgesen S, Ho CH, Irzyk GP, Jando SC, Alenquer ML, Jarvie TP, Jirage KB, Kim JB, Knight JR, Lanza JR, Leamon JH, Lefkowitz SM, Lei M, Li J, Lohman KL, Lu H, Makhijani VB, McDade KE, McKenna MP, Myers EW, Nickerson E, Nobile JR, Plant R, Puc BP, Ronan MT, Roth GT, Sarkis G], Simons JF, Simpson JW, Srinivasan M Tartaro KR, Tomasz A, Vogt KA, Volkmer GA, Wang SH, Wang Y, Weiner MP, Yu P, Begley RF and Rothberg JM: Genome sequencing in microfabricated high-density picolitre reactors. Nature 2005, 437:376-380.

2454. Shendure J, Porreca GJ, Reppas NB, Lin X, McCutcheon JP, Rosenbaum AM, Wang MD, Zhang K, Mitra RD and Church GM: Accurate multiplex polony sequencing of an evolved bacterial genome. Science 2005, 309:1728-32.

2455. Bentley DR, Balasubramanian S, Swerdlow HP, Smith GP, Milton I Brown CG, Hall KP, Evers DJ, Barnes CL, Bignell HR, Boutell JM, Bryant J, Carter RJ, Keira Cheetham R, Cox AJ, Ellis DJ, Flatbush MR, Gormley NA, Humphray SJ, Irving LJ, Karbelashvili MS, Kirk SM, Li H, Liu X, Maisinger KS, Murray LJ, Obradovic B, Ost T, Parkinson ML, Pratt MR, Rasolonjatovo IM, Reed MT, Rigatti R, Rodighiero C, Ross MT, Sabot A, Sankar SV Scally A, Schroth GP, Smith ME, Smith VP, Spiridou A, Torrance PE, Tzonev SS, Vermaas EH, Walter K, Wu X, Zhang L, Alam MD, Anastasi C, Aniebo IC, Bailey DM, Bancarz IR, Banerjee S, Barbour SG, Baybayan PA, Benoit VA, Benson KF, Bevis C, Black PJ, Boodhun A, Brennan JS, Bridgham JA, Brown RC Brown AA, Buermann DH, Bundu AA, Burrows JC, Carter NP, Castillo N, Chiara ECM, Chang S, Neil Cooley R, Crake NR, Dada OO, Diakoumakos KD, Dominguez-Fernandez B Earnshaw DJ, Egbujor UC, Elmore DW, Etchin SS, Ewan MR Fedurco M, Fraser LJ, Fuentes Fajardo KV, Scott Furey W, George D, Gietzen KJ, Goddard CP, Golda GS, Granieri PA, Green DE, Gustafson DL, Hansen NF, Harnish K, Haudenschild CD, Heyer NI, Hims MM, Ho JT and Horgan AM, et al: Accurate whole human genome sequencing using reversible terminator chemistry. Nature 2008, 456:53-9.
2456. Harris TD, Buzby PR, Babcock H, Beer E, Bowers J, Braslavsky I, Causey M, Colonell J, Dimeo J, Efcavitch JW, Giladi E, Gill J, Healy J, Jarosz M, Lapen D, Moulton K, Quake SR, Steinmann K, Thayer E, Tyurina A, Ward R, Weiss H and Xie Z: Single-molecule DNA sequencing of a viral genome. Science 2008, 320:106-9.

2457. Gupta PK: Single-molecule DNA sequencing technologies for future genomics research. Trends Biotechnol 2008, 26:602-II.

2458. Kahvejian A, Quackenbush J and Thompson JF: What would you do if you could sequence everything? Nat Biotechnol 2008, 26: $1125-33$.

2459. Zerbino DR and Birney E: Velvet: algorithms for de novo short read assembly using de Bruijn graphs. Genome Res 2008, 18:82I-9.

2460. Pavlopoulos GA, Wegener A-L and Schneider R: A survey of visualization tools for biological network analysis. Bio-data mining 2008, I:I2

246I. Garny A, Nickerson DP, Cooper J, Weber dos Santos R, Miller AK, McKeever S, Nielsen PM and Hunter PJ: CellML and associated tools and techniques. Philos Transact A Math Phys Eng Sci 2008 366:3017-43

2462. Li XJ, Brazhnik O, Kamal A, Guo D, Lee C, Hoops S and Mendes P: Databases and visualization for metabolomics. Metabolic profiling: its role in biomarker discovery and gene function analysis Kluwer Academic Publishers, Boston: Harrigan GG, Goodacre R 2003, 293-309.

2463. Jenkinson AM, Albrecht $M$, Birney $E$, Blankenburg $H$, Down $T$, Finn RD, Hermjakob H, Hubbard TJ, Jimenez RC, Jones P, Kähäri A, Kulesha E, Macias JR, Reeves GA and Prlić A: Integrating biological data - the Distributed Annotation System. BMC Bioinformatics 2008, 9(Suppl 8):S3.

2464. Köhler J, Baumbach J, Taubert J, Specht M, Skusa A, Ruegg A, Rawlings C, Verrier P and Philippi S: Graph-based analysis and visualization of experimental results with ONDEX. Bioinformatics 2006, 22: I 383-90.

2465. Pettifer SR, Sinnott JR and Attwood TK: UTOPIA - user-friendly tools for operating informatics applications. Comp Func Genom 2004, 5:56-60.

2466. McDermott P, Sinnott J, Thorne D, Pettifer S and Attwood T: An architecture for visualisation and interactive analysis of proteins. Proc 4th Int Conf Coordinated and Multiple Views in Exploratory Visualization (CMV06) 2006, 55-68.

2467. Thorne D, Pettifer S and Attwood T: Integrating Abstract and Physical Molecular Model Interaction. EG UK Theory and Practice of Computer Graphics (2005) Eurographics Association: Lever L, McDerby M 2005, 75-82.

2468. Pettifer S, Wolstencroft K, Alper P, Attwood T, Coletta A, Goble C, Li P, McDermott I, Marsh J Oinn T, Sinnott J and Thorne D: ${ }^{\text {my }}$ Grid and UTOPIA: an integrated approach to enacting and visualising in silico experiments in the life sciences. Proc Conf Data Integration in the Life Sciences 2007, 59-70.

2469. Neufeld El: Oral chelators deferasirox and deferiprone for transfusional iron overload in thalassemia major: new data, new questions. Blood 2006, 107:3436-4I.

\section{Pre-publication history}

The pre-publication history for this paper can be accessed here:

http://www.biomedcentral.com/1755-8794/2/2/prepub 